\title{
Numerical method for shock driven multiphase flow with evaporating particles
}

\author{
A Thesis presented to \\ the Faculty of the Graduate School \\ at the University of Missouri
}

In Partial Fulfillment

of the Requirements for the Degree

Master of Science

by
JEEVAN DAHAL

Dr. Jacob A. McFarland, Thesis Supervisor

DECEMBER 2016 
The undersigned, appointed by the Dean of the Graduate School, have examined the thesis entitled:

\section{NUMERICAL METHOD FOR SHOCK DRIVEN MULTIPHASE FLOW WITH EVAPORATING PARTICLES}

Presented by Jeevan Dahal, A candidate for the degree of Masters of Science, and hereby certify that, in their opinion, it is worthy of acceptance.

Dr. Jacob A. McFarland

Dr. Chung-Lung Chen

Dr. Angela Speck 


\section{ACKNOWLEDGMENTS}

I am very grateful to my advisor, Dr. Jocab A. McFarland, for his guidance and support throught the time of my master's research. A true genious, this work would not have been possible without his continuous insights and suggestions. He is a source of inspiration whose dedication to research and teaching has surely transformed me into a better researcher. His continuous trust in me to carry out this work has always been my source of motivation for working hard.

I would like to thank my committee members Dr. Chung-Lung Chen and Dr. Angela Speck for their input in this work. The meetings with Dr. Angela Speck increased my understanding of the applications of this work in astrophysics. Her guidance in defining the study problem and parameters were invaluable in the completion of this work. Dr. Chung Lung Chen, one of my first teacher at graduate school, increased my understanding and love to fluid mechanics that molded my motivation to continue working in the field.

I would like to thank my lab mate, Wolfgang Black, for his help in the reserach and in the class room. He is the first graduate student I met at Mizzou, and he helped me a lot to adapt to the Mizzou culture. Becuase of him I could quickly adapt to the life of a graduate student. His good work ethics constantly motivated me to work harder every day pushing me to the limits.

I would like to thank Constantine Avogustopalous and Roy Allen for helping me with the presentation and putting in words of encouragement when I have been through tough days. Giwen Quo and Justin White for increasing my understanding of the fundamentals of subject matters asking tough questions and engaging in the conversations. John MiddelBrooks for his support and Rajya Varthan Reddy Kutthakopa for being a nice friend who is always ready to help.

I would like to take this opportunity to thank my old friends, who along the 
way have helped me to be the man I am today. My friend and current lab mate Manoj Paude for teaching me to be optimistic and look on the bright side while going through the rough times. Sanjib K C for being a caring friend and sharing my struggle and success. Prabhas Ghimire, Sashank Dewan, Pallab shrestha, Sharad Raj Acharya, Saroj Paudel, and Prasanna Thapa Magar for always being there when I needed some words of encouragement.

Last but not the least, I would like to thank Priyanka Shahi, the most humble person I have met in my life, for being always by my side during the ups and downs of the life. I would like to thank her for trusting in me and motivating me to help realise my potential in being a better human being. This journey would never have been complete if she was not by my side. 


\section{TABLE OF CONTENTS}

ACKNOWLEDGMENTS .................. ii

LIST OF TABLES .................... vii

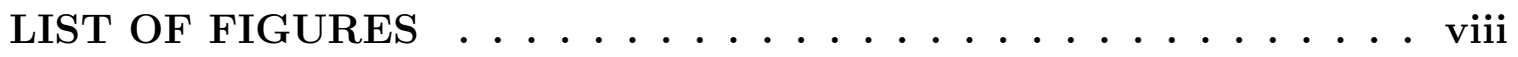

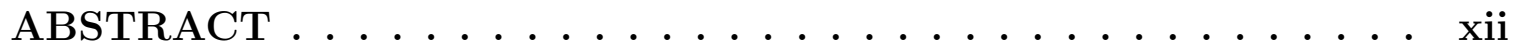

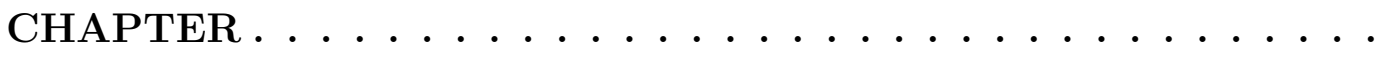

1 Introduction ...................... 1

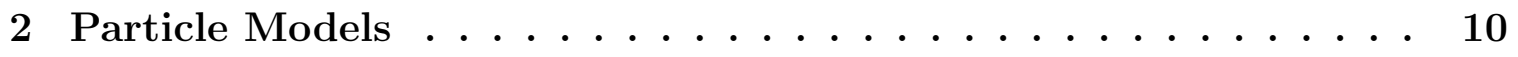

2.1 Methods . . . . . . . . . . . . . . . . . . . 10

2.1.1 Governing equations ... . . . . . . . . . . . . 10

2.1.2 Interpolation method . . . . . . . . . . . . 19

2.1.3 Momentum transfer . . . . . . . . . . . . . . . . 20

2.1.4 Energy transfer . . . . . . . . . . . . . . 25

2.1.5 Mass transfer by evaporation . . . . . . . . . . . . . 27

2.1.6 Implementation in the FLASH code . . . . . . . . . . . . . . 28

2.2 Validation . . . . . . . . . . . . . . . . . . 32

2.2.1 Particle temperature and velocity history . . . . . . . . . . . 32

2.2.2 Particle evaporation properties . . . . . . . . . . . 35

2.2.3 Comparison to experiments . . . . . . . . . . . 36

3 Simulation study in 2D ...................... 42

3.1 Single particle enstrophy . . . . . . . . . . . . . . . . . . 42

3.2 Simulation with evaporating particles . . . . . . . . . . . . 48

3.2.1 Qualitative effects ................. 48 
3.2 .2 Quantitative effects . . . . . . . . . . . . . . . . 49

3.2 .3 Particle property histories $\ldots \ldots \ldots \ldots \ldots$

3.2.4 Late time qualitative comparison . . . . . . . . . . 54

4 Multiphase hydrodynamic instability in $3 \mathrm{D} \ldots \ldots \ldots \ldots$

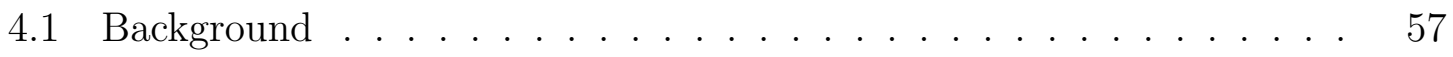

4.2 Simulation setup $\ldots \ldots \ldots \ldots \ldots \ldots \ldots$

4.3 Qualitative analysis . . . . . . . . . . . . . . . . 62

4.4 Quantitative analysis . . . . . . . . . . . . . 67

5 Summary and Conclusions . . . . . . . . . . . . 69

5.1 Acknowledgments . . . . . . . . . . . . . . . . . . 72

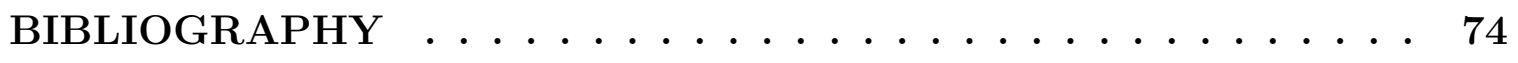

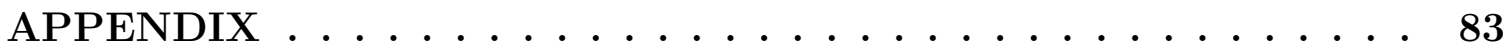

A Particle Models and Subroutines . . . . . . . . . . . 83

A.1 Momentum Exchange . . . . . . . . . . . . . . . . 83

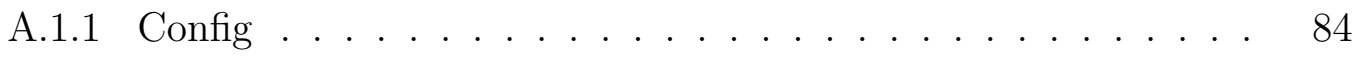

A.1.2 Makefile ... . . . . . . . . . . . . . . 87

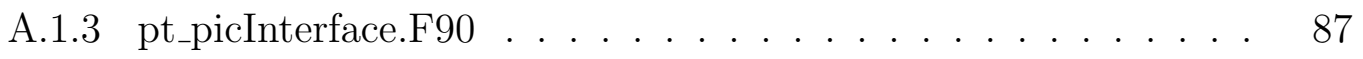

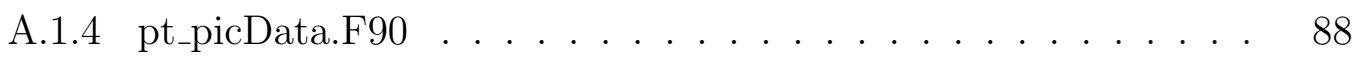

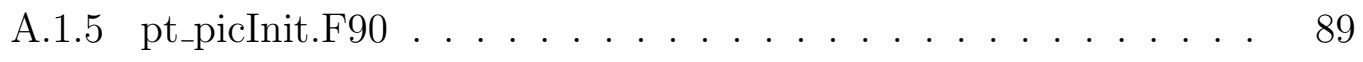

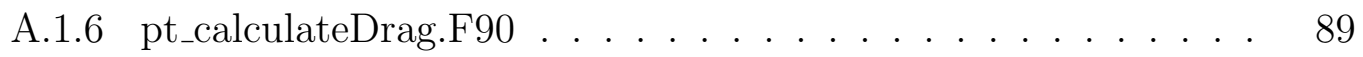

A.1.7 pt_calculateVar.F90 . . . . . . . . . . . . . . . . . . . 93

A.1.8 pt_picAdvanceVel . . . . . . . . . . . . . . . . . . . . . . . . . 98

A.1.9 pt_advanceCharged.F90 _ . . . . . . . . . . . . . . 100

A.1.10 pt_calculateVarTemp . . . . . . . . . . . . . . . . . . 101

A.1.11 pt_calculateTemp . . . . . . . . . . . . . . . . . . . 102 
A.2 Energy Exchange . . . . . . . . . . . . . . . . . . . . . 103

A.2.1 Config . . . . . . . . . . . . . . . . . . 103

A.2.2 Makefile . . . . . . . . . . . . . . . . . . . . . 104

A.2.3 pt_picData. . . . . . . . . . . . . . . . . . . . . . . . 104

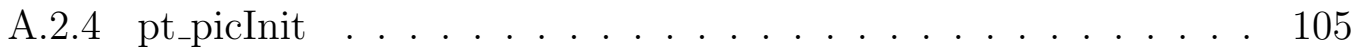

A.2.5 pt_calculateVarTemp.F90 . . . . . . . . . . . . 105

A.2.6 pt_calculateTemp.F90 . . . . . . . . . . . . . . . . . . . . . . 109

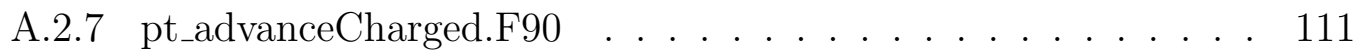

A.3 Phase Change . . . . . . . . . . . . . . . . . . . . . . 113

A.3.1 Config . . . . . . . . . . . . . . . . . . . . 113

A.3.2 pt_picData. . . . . . . . . . . . . . . . . . . . . . . . . 114

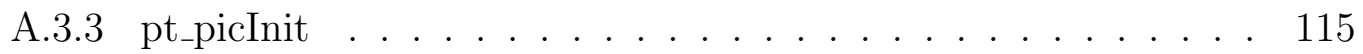

A.3.4 pt_initSpecies . . . . . . . . . . . . . . . . . 116

A.3.5 pt_calculateVarTemp . . . . . . . . . . . . . 116

A.3.6 pt_calculateTemp . . . . . . . . . . . . . . . . . . . . . . . . 121

A.3.7 pt_advanceCharged . . . . . . . . . . . . . . . . . . . . . . . . 124

A.4 Hydro . . . . . . . . . . . . . . . . . . . 127

A.4.1 Hydro.F90 . . . . . . . . . . . . . . . . . . . . . 127

A.4.2 hy_ppm_updateMom.F90 . . . . . . . . . . . . . . . 132

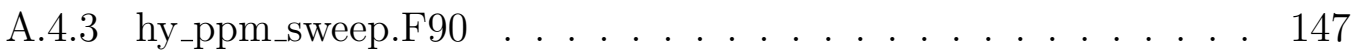




\section{LIST OF TABLES}

Table

2.1 Antoine coefficients for water $\ldots \ldots \ldots \ldots \ldots$

2.2 Radius, number of particles per parcel and momentum relaxation time for different groups . . . . . . . . . . . . . . . . . 38

3.1 Radius and number of particles per parcel of different groups for evaporating particles . . . . . . . . . . . . . . . . 49 


\section{LIST OF FIGURES}

Figure

2.1 Numerical algorithm for the implementation of the particle models in

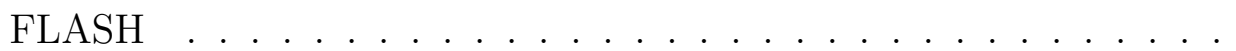

2.2 Single particle validation of the temperature history of the particle model in FLASH code with the analytical Solution. The line in the figure represents the analytical solution results and the markers represents the FLASH code results. . . . . . . . . . . . . . .

2.3 Single particle validation of the velocity history of the particle model in FLASH code with the analytical Solution. The line in the figure represents the analytical solution results and the markers represents the FLASH code results. . . . . . . . . . . . . . . . .

2.4 Time history of the gas temperature and vapor concentration in air due to particle evaporation. The primary axis is temperature $(\mathrm{K})$ and the secondary axis is concentration. . . . . . . . . .

2.5 Initial condition for the simulation . . . . . . . . . . . .

2.6 Left: Distribution of the particles in the air cylinder. Right: Four cells are magnified to show the arragement of particles in a cell . . . . . 
2.7 Comparison of of the FLASH simulation of the air cylinder with glycol particles of single group and multi-group with that of the Vorobieff experiments. The shock strength is $M=1.65$ and the average radius of the particle is $1 \mu \mathrm{m} . t=0$ corresponds to the time when the shock wave is at the center of the gas cylinder. Top: Vorobieff experimental results adapted with permission from [1], Copyrighted by the American Physical Society". Center: FLASH simulation of the multi-group of particles consisting of five different groups of radius $0.4 \mu \mathrm{m}, 0.9 \mu \mathrm{m}$, $1.2 \mu \mathrm{m}, 1.6 \mu \mathrm{m}, 2.2 \mu \mathrm{m}$. Bottom: FLASH simulation with single group of particles of radius $1 \mu m . \ldots \ldots \ldots$

2.8 Circulation plot of the single group and multi-group particles without evaporation ..................... . . 41

3.1 Circulation plot for $1 \mu m$ and $5 \mu m$ single particle . . . . . . . . .

3.2 Enstrophy plot for $1 \mu m$ and $5 \mu m$ single particles. The solid line gives the actual enstrophy on the gas and the dotted line shows the enstrophy deposited by the source term . . . . . . . . . . . .

3.3 Time rate of enstrophy for $1 \mu \mathrm{m}$ and $5 \mu \mathrm{m}$ particle. Solid line gives the actual rate of enstrophy deposition and the dotted line shows the rate of enstrophy deposition by source term . . . . . . . . . . .

3.4 Rate of enstrophy dissipation for $5 \mu m$ and $1 \mu m$ particle. The solid line is the actual enstrophy dissipation plotted in the primary $Y$ axis and the dotted line is the enstrophy dissipation considering the viscosity of the gas plotted on secondary $Y$ axis . . . . . . . . . . . . . 46

3.5 Effective viscosity for the $1 \mu m$ and $5 \mu m$ particle size . . . . . . . 47 
3.6 Plot of FLASH simulation of the single group and multi-group of particles at $M=1.65$ with evaporation. The particles are plotted on top of evaporated vapor species with different color bars. Top: evaporating multi-group of particles with radius $0.4 \mu m, 0.9 \mu m, 1.2 \mu m, 1.6 \mu m$, $2.2 \mu \mathrm{m}$. Bottom: evaporating single group of particles with radius $1 \mu \mathrm{m} 50$

3.7 Circulation plot of the single and multiple group of particles with and without evaporation. The solid lines shows the plot without evaporation and the dotted lines are for the plots with evaporation . . . . . 51

3.8 Enstrophy plot of the single and multiple group of particles with and without evaporation. The solid lines shows the plot without evaporation and the dotted lines are for the plots with evaporation . . . . . 51

3.9 Time history of the droplet diameter $\ldots \ldots \ldots \ldots \ldots$

3.10 Time history of the rate of change of droplet diameter $\ldots \ldots \ldots$

3.11 Time history of the droplet temperature $\ldots \ldots \ldots \ldots$

3.12 Time history of the gradient of droplet temperature . . . . . . 54

3.13 Pseudocolor plots at $\tau=2230 \mu \mathrm{s}$. Top: Non-evaporating multiple group particles. Top Center: Non-evaporating single group particles. Bottom center: Evaporating multiple group of particles. Bottom: Evaporating single group of particles. Left: Particle radius on top of the vapor species. Left center: Gas density field. Right center: Temperature of the gas. Right: Vorticity field . . . . . . . . . . 56

4.1 Histogram of the particles radii at initialization $\ldots \ldots \ldots$. . . . 61

4.2 Intial setup of the 3D case showing the pressure contour and tagged gas contour containing the particles. . . . . . . . . . . 
4.3 Contour plots of gas parameters with particles. Top: Counter plot of vapor fraction with slice at the center plane in $\mathrm{Z}$ direction for both the gas and particles. Bottom: Counter plot of the tagged gas with reduced opacity to visualize the particles in the center and the slice of the particles in the $\mathrm{Z}$ direction at the center plane. . . . . . . . . .

4.4 Pseudocolor plots of gas properties at various time. The plot is sliced at the centerplane in the $Z$ direction $(X Y$ plane $) . \quad \ldots . . . .$.

4.5 Vorticity plot at $\mathrm{t}=1.3 \mathrm{~ms}$. The domain is sliced at the centerplane in $Z$ direction $(X Y$ plane $) . \ldots \ldots \ldots \ldots$

4.6 Vorticity plot at $\mathrm{t}=1.3 \mathrm{~ms}$. The domain is sliced at different locations in the X direction. . . . . . . . . . . . . . . . 66

4.7 Circulation plots in $\theta$ and $x$ direcions. . . . . . . . . . . . . 67

4.8 The width of the particle interface with time. . . . . . . . 68 


\section{ABSTRACT}

This work is done in part as a requirement towards the fulfillment of the master's degree. Four different chapters were explored in this work. Chapter 1 gives the overview of the various works that have been done in the field and the applications of this work. Chapter 2 discusses the details of the numerical methods developed for predicting the interaction of active, phase changing particles in a shock driven flow. The Particle-in-Cell (PIC) technique was used to couple particles in a Lagrangian coordinate system with a fluid in an Eulerian coordinate system. This method was implemented in the open source hydrodynamics software FLASH, developed at the University of Chicago. A simple validation of these methods is accomplished by comparing particle properties at advance time qualitatively with the analytical solution and quantitatively with the experiments.

Chapter 3 explores the parametric study of the multiphase hydrodynamics. Here, we are particularly interested in the effects of paricle size distribution and the particle radius during evolution of multiphase hydrodynamic instability. It is found that the distribution of particles sizes causes less vorticity deposition than a case which considers only single size of same median diameter. The large particles are found to have lower enstrophy production at early times and higher enstrophy dissipation at late times due to the advection of the particle vorticity source term through the carrier gas resulting in less net overall vorticity depositon. Furthermore, the particle evaporation is found to increase the vorticity deposition causing the interface to evolve faster.

Chapter 4 expands upon the study done in the previous chapter to the third dimension. Further validation of the code is done to make sure that the results obtained in the third dimension are correct. Shock interaction of the particle sphere

containing randomly selected particle radii, which follow a lognormal distribution of 
median $1 \mu m$ at, random locations within the sphere is studied. This simulation is run with about 1 million particles at 1:1 parcel to particle size, and resolution of 128 nodes in the circle. The qualitative study shows the initial particle lag making the interface width large at early time. After some time, the hydrodynamic growth is found to overcome the differences in the particle velocity relaxation time decreasing the interface width. A highly asymmetric gas field is seen with alternating patches of positive and negative vorticity in the vorticity field.

Further analysis of the 3D data will be done in the future to characterise the particle turbulence characteristics in the shock driven condition. These turbulence data would be interpreted to get new insights on the behaviour of the cosmological dust during the shock interactions. 


\section{Chapter 1}

\section{Introduction}

This work is done in part as a requirement towards the fulfillment of the master's degree. In this work, a numerical model for the solution of the interaction of active phase changing particles in the gas medium is developed. In chapter 1, a brief introduction of the various numerical methods used by other researchers is given and the applications and importance of these models is highlighted. In chapter 2, the detailed mathematical equations describing the particle models are developed and validated. In chapter 3 , these models are used to study the effect of particle drag and particle evaporation on the evolution of the multiphase hydrodynamic instability, and chapter 4 expands upon this study with the help of three dimensional shock driven multiphase simulations.

The advent of high performance computing has made possible the study of various multiphase problems by using numerical methods. In this work we focus on the gasparticle flow, a type of multiphase flow, which appears in a large number of natural, biological and industrial processes such as cosmic dust processing in supernovae and Asymptotic Giant Branch stars, shock processed water droplets in air and steam turbines, supersonic liquid fuel combustion, volcanic ash production, nuclear and biological agent particulate dispersion, and solid propellant rockets to name a few. In 
multiphase flow, the phase containing higher volume is called the carrier phase and the phase containing smaller volume is called the dispersed phase.

Multiphase flow in the laminar regime can be analysed by using basic equations. However, as the flow transitions to turbulence, it requires greater attention. The Reynolds number characterises the regime of the fluid flow; laminar, transition, or turbulence. The Reynolds number is the ratio of the inertial force to the viscous force and is given by equation 1.1.

$$
R e=\frac{\rho_{g} \mathbf{v}_{\mathbf{g}} d}{\mu_{g}}
$$

In equation $1.1, \rho_{g}$ is the density of the flow, $\mathbf{v}_{\mathbf{g}}$ is the velocity of the flow, $d$ is the characteristic dimension, and $\mu_{\mathbf{g}}$ is the dynamic viscosity of the fluid.

The critical Reynolds number, $R e_{c r}$, gives the transition of the laminar flow towards turbulence. In the transition regime the solutions of all the small scale perturbations are stable. The number of solutions continuously increase with the increasing Reynolds number and at a certain point the small scale perturbations grow exponentially causing the flow to abrutly transition to turbulence. The turbulence is characterized by the chaotic, random fluctations in the velocity profile. The turbulent flow cannot be solved analytically and is characterized by using statistical methods.

The transition of a fluid profile from a hydrodynamically stable region to turbulence is called hydrodynamic instability. For the development of the hydrodynamic instabilty three ingredients are required: an interface, perturbations at the interface, and the mechanism for the growth of the interace. The mechanism for the growth can be constant acceleration, velocity shear, a shock wave or others. Based on the mechanism, typically three types of hydrodynamic instabilities can arise in this work: the Rayleigh Taylor Instability (RTI) [2, 3], Kelvin Helmholtz Instability (KHI) [4], and Richtmyer Meshkov Instability (RMI) [5, 6]. In the RTI the interface evolves towards the instability regime due to constant gravitational force, which typically occurs in nature when there is a heavy fluid over the light fluid. One example of it is a brine 
water solution on top of the fresh water. It also causes various phenamenon occuring in nature such as the finger like structures seen at the interface of the supernovae remnant during the supernovae explosion [7], and diapiric ascent of salt through the densar overburden [8]. In the KHI the interface evolves due to velocity shear which occurs in nature during atmoshperic cloud formation where the interface between the cloud and the air is driven to form vorticle structures [4]. In the RMI, the interface is driven by a shock wave which gives rise to an instantaneous acceleration as opposed to the constant acceleration in RTI. The misalignment between the density gradient and the pressure gradient gives rise to a baroclinic term in the vorticity equation forming vortical structurs. The vorticity equation is given as:

$$
\frac{D \omega}{D t}=\omega \cdot \nabla \mathbf{v}_{\mathbf{g}}+\nu_{g} \nabla^{2} \omega+\left[\frac{1}{\rho_{g}^{2}} \nabla \rho_{g} \times \nabla P\right]_{\text {baroclinicterm }}
$$

In equation $1.2, \omega$ is the vorticity of the flow, $\nu_{g}$ is the kinematic viscosity, and $P$ is the pressure.

The RMI occurs in inertial confinement fusion where it decreases the yield of energy by driving the mixing between the layers of the fuel capsule [9]. In atrophysics, it appears during the supernovae explosion where a blast wave drives mixing together with the RTI. Particles also drive an instability called the shock driven multiphase instability (SDMI). We are interested in a multiphase hydrodynamic instability whose evolution is similar to that of RMI [1].

The SDMI arises in various cases in nature. It is required to study the shock processing of the cosmic dust. Cosmic dust plays an important role in the evolution of the galaxies and stars. Cosmic dust is produced by the supernove explosion events and the Asymptotic Giant Branch (AGB) stars. The dust in the circumstellar shells is destroyed by evaporation, splitting, sputtering, collision, and sublimation due to the forward and reverse shock interactions $[10,11,12]$. It is also involved in the study of the dynamic properties of shock induced ejecta particles in the case of high energy 
explosives [13]. For the targets that remain solid after the shock wave releases, small fragmants release from the surface defects and inhomogeneities of the metal [14]. In the case of melting of the metal during shock compresion and release, a high energy fragmant layer that enhances ejecta production forms on the metal surface [15]. Likewise, the complex interaction of the particles with gases and liquids occurs in volcanic eruptions resulting in the formation of hydrovolcanic pyroclasts (tephra) [16]. The hydrodynamic interactions together with the ratio of condensation, evaporation, and breaking determines the size distribution of the pyroclasts. Another application is the shock interaction of the fuel and oxidizer in the combustion chamber of scramjet engines which helps in mixing by breaking up the droplets and driving particle evaporation [17]. Supersonic combustion has a short residence time, of the order of $1 \mathrm{~ms}$, and thus requires a rapid mixing of the air and fuel. SDMI also has applications in steam turbines and ejector pump refrigeration cycles, where, liquid droplets condense and interact with shock wave $[18,19]$.

Multiphase flow can be either dilute or dense. In dilute flow, particle motions are controlled by fluid forces whereas in dense flow, particle motions are controlled by collisions. In the dilute flow, the particle volume fraction, $\theta$, is low and the interaction between the particles are not important as their behavior is dominated by the gas characteristics. Collisions are important in flows with volume loading of above $5 \%$ [20]. Dense flows and particle collisions are important in the study of granular flows and sedimentation. In most multiphase particle in cell (MP-PIC) models, collisions are modelled by an interparticle stress term given by equation 1.3 .

$$
\tau=P \frac{\theta}{\theta_{c p}-\theta}
$$

In equation $1.3, \tau$ is the interparticle stress term, $P$ is the pressure, $\theta$ is the particle volume fraction, and $\theta_{c p}$ is the particle volume fraction at the close packing limit. The close packing limit is a condition where the volume fraction of a grid is such that no 
additional particles can enter the grid. Our study is mostly based on small particles whose volume loading is very small. As such, we ignore the effect of particle collisions and interparticle stress in this study.

Balachandar and Eaton [21] provide a helpful review of the concepts important to mixing in turbulent multiphase flows. Various modeling techniques have been formulated to study these problems which can be broadly classified into three types on the basis of the representation of the gas and particle phases: Eulerian-Eulerian, Eulerian-Lagrangian, and Lagrangian-Lagrangian.

In the Eulerian-Eulerian approach, the particles are considered to be another fluid which continuously interacts with the gas phase. In this approach, a solution is obtained by six partial differential equations (conservation equations) that are discretized on the grid, three each for the particle and gas phase. The model given by Gidaspow [22] for fluidization uses this approach. This model has many attractive advantages for modeling dense particle flows as it allows one to calculate the spatial gradient of the volume fraction on the grid which makes for an easier calculation of the inter-particle stress [23]. However, if we want to include more than one particle size each particle size must be represented as a phase. The addition of each phase will introduce three additional conservation equations increasing the complexity of the calculations.

Eulerian-Lagrangian approach for modeling multiphase flows are popular as it is more convenient to represent particles in the Lagrangian coordinate system. Such a representation requires solving the fluid conservation equations on the Eulerian grid with particle source terms to introduce the effect of particles to the fluid flow. The source terms for mass, momentum and energy are computed by solving the particle Lagrangian equations with the help of fluid properties interpolated to their position. The particle-in-cell (PIC) approach introduced by Evans et.al [24] uses the Lagrangian representation of particles in an Eulerian grid and has been improved 
upon by others. The multiphase particle-in-cell approach (MP-PIC) given by Andrews and O'rourke [20] is an extension the PIC approach, and has both the Eulerian and Lagrangian representation of particles. This additional continuum equation allows fully implicit coupling of the particle phase with the gas phase equations giving a stable and accurate solution. The MP-PIC method was extended to three dimensions using trilinear interpolation by Snider [25]. These MP-PIC implementations make the assumption that the flow is essentially inviscid and viscosity is considered only at particle location when calculating drag.

Patankar and Joseph [26] improved on the previous numerical methods by taking into account the fluid viscosity while solving the governing equations. In addition, they used the pressure correction based fractional-step time stepping technique. These numerical methods were developed to simulate dense particulate solid particle flows like sedimentation, flow in fluidized beds, and slurries where only the momentum of the particle is of importance. Sinder et at. [27] gave a three dimensional Eulerian-Lagrangian method with energy and mass transfer involving chemical reactions using the computational particle fluid dynamics (CPFD) methodology. Another MP-PIC based method was implemented in the computer program KIVA, and used to model chemically reactive flows with sprays. This method uses the stochastic particle method [28, 29]. Based on the KIVA code, Cloutman [30] developed a simpler method that can handle particle momentum and energy exchange, but did not include an evaporation model. Various studies have been undertaken to develop a particle evaporation model which accounts for the non-linear effects in evaporation. In an effort to find the most computationally efficient and accurate model for predicting the droplet diameter, a number of popular droplet evaporation models have been compared and summarized by Miller et al. [31].

Some popular Lagrangian-Lagrangian models have been developed and extensively used in numerical simulations that represent the fluid as packets of particles. The 
smoothed particle hydrodynamics (SPH) method discussed by Monaghan [32] and moving particle semi-implicit method given by Kashizyka and Oka [33] are two of these methods. The representation of the fluid in the Lagrangian coordinate system has a number of attractive advantages. It can solve for pure advection accurately giving a numerical dissipation free solution, it can guarantee the conservation of momentum and energy, it can simulate complex geometries, and it provides easy coupling of the particle and gas phases. $\mathrm{SPH}$ is popular in the astrophysics community as it is computationally efficient since the calculations are done only in the areas where high amount of mass are present. The SPH method has been compared to Eularian methods for shock driven gas only flows, similar to the one investigated in this paper, by Stanic et al. [34].

Shock-driven multiphase flows are similar in many ways to the RMI and can arise in the study of this instability as particles are used as passive tracers for the gas phase. Balakumar et al. [35] used particle imaging velocimetry (PIV), with fog particles as the tracer, along with laser induced fluorescence of acetone vapor to get simultaneous velocity and concentration measurements in a reshocked turbulent RM flow. Jacobs et al. [36] used incense smoke to mark one species and then used Mie scattering of laser light from the smoke particles to track the gas location during RMI experiments. Haehn et al. [37] used liquid particles up to $25 \mu \mathrm{m}$ in diameter created by the destruction of a soap film membrane to get PIV measurements of the velocity field in a shock bubble interaction. McFarland et al. [38] used sub-micron diameter glycerol particles to track gases using the Mie scattering technique in inclined interface RM experiments. Recent work from Reilly et al. [39] presented a 2D field of uncertainty in velocities calculated using PIV measurements with sub-micron glycerol particles and McFarland et al. [40, 41] presented simulations of a similar interface. The work of Vorobieff et al. [1] explored the effect of particles in the RMI and first identified that an instability could be created by the interaction of a shock wave with a multi-phase 
system interface alone. In this paper, the authors showed that a cloud of micron sized particles could induce an instability and mixing similar to a RMI. Further work by Anderson et al. [42] investigated the role of particles in a shock-cylinder RMI with experiments and simulations. Their work showed good qualitative agreement between particle-in-cell (PIC) simulations and experiments.

A number of other simulation studies have been done to study the effect of particles in the RMI. Ukai et al. [43] studied the RMI with particles both numerically and analytically and derived a growth model for multiphase effects in a RMI using the dusty gas approximation. Schulz et al. [44] extended the study performing 3D simulations of a dilute gas-particle mixture before and after reshock with various initial conditions. McFarland et al. [45] performed a parametric study of the shock-driven multiphase instability highlighting the effects of gas-particle coupling, incident shock strength, particle size, and multiple particle relaxation time effects. Multiphase flows have various astrophysical applications as particles are present as cosmic dust. Miura et al. [46] numerically simulated the heating process in the passage of a shock wave through a silicate particle field taking into account evaporation to study the formation of chondrules from precursor particles. Van Marle et al. [47] investigated the behavior of the turbulent interaction between dust grains in circumstellar and interstellar space separated by the contact discontinuity of a bow shock formed by a fast moving red supergiant. Silvia et al. [11] used passive Lagrangian particles representing dust grains to study dust destruction by the reverse shock in supernovae (SNe) remnants.

In this study, a new numerical method for shock-driven multiphase flows is developed by combining the PIC method with the piecewise parabolic method(PPM) given by Colella and Woodward [48] for compressible flows. Since many applications of SDMIs involve dilute flow with particles, the model given is designed only to simulate particle concentrations below the close pack limit. A simple and efficient energy transfer model, momentum transfer model with drag correlations, and evaporation 
model for water droplets is given to predict particle location, their effect on carrier phase, their diameters and temperatures. The models are implemented in the open source hydrodynamics code called FLASH developed and maintained by theFlash Center for Computational Science at University of Chicago.

Chapter 2 discusses various numerical methods that were used for solving particle conservation equations and coupling them with the PPM using the PIC technique. The models are validated with the analytical solutions and experimental results found in the literature. In chapters 3 and 4, the particle models are used to simulate shock interactions with the particles with various initial conditions. We also try to draw a connection between our results and relevance with astrophysical phenomenon. In addition, added capabilities that would allow our models to predict astrophysical dust production and destruction will also be discussed. 


\section{Chapter 2}

\section{Particle Models}

\section{$2.1 \quad$ Methods}

In this chapter the author describes the details of the governing equations, interpolation method, and the numerical method that was used for the new particle models. The contents of this and the following chapter are from the paper "Numerical method for shock driven multiphase flow with evaporating particles" which is currently under review with the Journal of Computational Physics.

\subsubsection{Governing equations}

The piecewise parabolic method (PPM), a hydrodynamics solver developed by Colella and Woodward [48], is used for solving the conservation equations. For our work, the implementation of the PPM given by Fryxell et al. [49] is used. This implementation solves the Euler equations for compressible gas hydrodynamics in one, two, or three dimensions. The Euler equations are modified to account for the effect of the presence of particles by using a fluid volume fraction or void fraction, $\epsilon$, as given by the following equations, and particle source terms. 


$$
\begin{gathered}
\frac{\partial \epsilon \rho_{g}}{\partial t}+\nabla \cdot \epsilon \rho_{g} \mathbf{v}_{\mathbf{g}}=-M_{s} \\
\frac{\partial \epsilon \rho_{g} \mathbf{v}_{\mathbf{g}}}{\partial t}+\nabla \cdot \epsilon \rho_{g} \mathbf{v}_{\mathbf{g}} \mathbf{v}_{\mathbf{g}}+\nabla P=-F_{s}+\epsilon \rho_{g} \mathbf{g} \\
\frac{\partial \epsilon \rho_{g} E}{\partial t}+\nabla \cdot\left(\epsilon \rho_{g} E+P\right) \mathbf{v}_{\mathbf{g}}+\frac{P \partial \epsilon}{\partial t}=\epsilon \rho_{g} \mathbf{v}_{\mathbf{g}} \cdot \mathbf{g}+E_{s}
\end{gathered}
$$

In the above equations, $\rho_{g}$ is the density of the gas, $\mathbf{v}_{\mathbf{g}}$ is the velocity of the gas, $E$ is the total energy of the gas, $\mathbf{g}$ is acceleration due to gravity, $P$ is pressure, $M_{s}$ is the mass source term, $F_{s}$ is the momentum source term, and $E_{s}$ is the energy source. These source terms are the contribution of the change in particle mass, momentum, and energy to the gas. In the PIC method, the particle source terms are obtained by solving the Lagrangian conservation equations. The communication between the gas and particles is made by these source terms coupled to the gas conservation equations.

These conservation equations are first solved in one direction and then the multidirection implementation is done by using directional splitting or Strang splitting. In the one dimensional implementation, fluxes entering and leaving through the $\zeta$ face of the zone are solved, where $\zeta$ is the direction in which the solution is being calculated. These fluxes have a contribution to the momentum of the fluid in the direction perpendicular to $\zeta$ by the advection term which is calculated using equations 2.6 and 2.7. Scalar fields like energy and mass are straight forward and are only affected by the fluxes in the solution direction as given by equations 2.4 and 2.8. The evaporated vapor from the particles contributes to the mass source term by increasing the overall mass of the vapor phase and hence the density of the gas. Hence, in the given conservation equations the mass source term, $M_{s}$, appears twice, once updating the density of the gas in a cell as given by equation 2.4 and once updating the vapor fraction, 
$X_{v s}$, of the evaporated vapor phase as given by equation 2.10 .

$$
\begin{gathered}
\frac{\partial \epsilon \rho_{g}}{\partial t}+\frac{\partial A \epsilon \rho_{g} u_{g}}{\partial V}=-M_{s} \\
\frac{\partial \epsilon \rho_{g} u_{g}}{\partial t}+\frac{\partial A \epsilon \rho_{g} u_{g}^{2}}{\partial V}+\frac{\partial P}{\partial \zeta}=\epsilon \rho_{g} g-F_{s} \\
\frac{\partial \epsilon \rho_{g} v_{g}}{\partial t}+\frac{\partial A \epsilon \rho_{g} u_{g} v_{g}}{\partial V}=0 \\
\frac{\partial \epsilon \rho_{g} w_{g}}{\partial t}+\frac{\partial A \epsilon \rho_{g} w_{g} u_{g}}{\partial V}=0 \\
\frac{\partial \epsilon \rho_{g} E}{\partial t}+\frac{\partial\left(A \epsilon \rho_{g} E+P\right) u_{g}}{\partial V}+\frac{P \partial \epsilon}{\partial t}=\epsilon \rho_{g} u_{g} g+E_{s} \\
\frac{\partial \epsilon \rho_{g} X_{v s, g}}{\partial t}+\frac{\partial A \epsilon \rho_{g} X_{v s, g}}{\partial V}=-M_{s} \\
\frac{\partial t}{\partial t}+\frac{\partial A \epsilon \rho_{g} X X_{l, g}}{\partial V}=0, \quad f o r \quad l \neq v s
\end{gathered}
$$

In the above equations, $u$ defines the component of the velocity in the $\zeta$ direction and $v$ and $w$ are the velocities perpendicular to $\zeta . X_{l}$ represents the mass fraction of the species given by index $l, A$ is the cross section area of the of the zone face in the $\zeta$ direction, $V$ is the volume of the cell, and $g$ is the gravitational acceleration in the $\zeta$ direction.

The PPM follows a series of steps to obtain the solution: i) reconstruction, ii) contact discontinuity steepening, iii) flattening, and iv) solution. These steps ensure that the algorithm can effectively detect shocks, smooth the gradients that are not caused by shock waves, maintain monotonicity, and ensure the accuracy and stability 
of the solution. More about these steps is given by Fryxell et al. [49].

For the particle phase equations, the author uses the Liouville equation for the particle distribution function $h\left(\mathbf{x}_{\mathbf{p}}, \mathbf{v}_{\mathbf{p}}, \rho_{p}, t\right)$ as given by equation 2.11 .

$$
\frac{\partial h}{\partial t}+\nabla_{\mathbf{x}_{\mathbf{p}} \cdot} \cdot\left(h \mathbf{v}_{\mathbf{p}}\right)+\nabla_{\mathbf{v}_{\mathbf{p}}} \cdot\left(h A_{p}\right)=0
$$

Where, $\mathbf{x}_{\mathbf{p}}$ is the particle position, $\mathbf{v}_{\mathbf{p}}$ is the particle velocity and $A_{p}$ is the particle acceleration which is given by equation 2.12

$$
A_{p}=D_{p}\left(\mathbf{v}_{\mathbf{g}}-\mathbf{v}_{\mathbf{p}}\right)-\frac{1}{\rho_{p}} \nabla_{\mathbf{x}_{\mathbf{p}}} P+\mathbf{g}-\frac{1}{\theta \rho_{p}} \nabla_{\mathbf{x}_{\mathbf{p}}} \tau_{p}
$$

In equation $2.12, \tau_{p}$ is the isotropic solid stress term, $\rho_{p}$ is the density of the particle material, $P$ is the gas pressure, and $D_{p}$ is the drag experienced by the particles. $\tau_{p}$ prevents the particles from reaching the close pack limit, which is the condition when $\epsilon$ of the cell is such that no additional particles can be enter the cell. The PIC method breaks when this condition occurs. The effect of $\tau_{p}$ is neglected as the flow is considered to be essentially dilute and unable to reach the close pack limit in shock loading conditions. $\tau_{p}$ is important for the solution of sedimentation and fluidized bed problems as they involve larger particles that tend to settle under gravity and accumulate. In the shock driven environment, we study the effect of particles of a micrometer size with very small value for $\theta$.

The drag experienced by the particle, $D_{p}$, is given by equation 2.13 , where $r_{p}$ is the radius of the particles and $C_{d}$ is the drag coefficient.

$$
D_{p}=C_{d} \frac{3}{8} \frac{\rho_{g}}{\rho_{p}} \frac{\left|\mathbf{v}_{\mathbf{g}}-\mathbf{v}_{\mathbf{p}}\right|}{r_{p}}
$$

Various research has been done to find the best drag coefficient for the shock tube environment. Parmar et al. [50] gave an improved correlation for the drag coefficient of a sphere in shock tube environment which was validated by the experiments of 
Jourdan et al. [51]. The drag correlation for a Reynolds number, Re, below several hundred can be well approximated by the equation given by Kilachko as given by Fuks [52]. Experiments done by Rudinger [53] showed that there is an error in calculating the drag coefficient using the Kliachko model in a shock tube for low Reynolds numbers and they provided a corrected drag model. Another correction to the standard drag model using a function of Mach number, $M$, is provided for a spherical particle by Carter et al. [54]. The Kliachko drag equation was modified by O'rourke [55] to include the effect of the particle volume fraction. This modified drag equation, given by equation 2.14, with the correction made by Carter et al. [54] for shock strength, given by equation 2.16 , is used in our method.

$$
\begin{gathered}
C_{d}=\frac{24}{R e}\left(\epsilon^{-2.65}+\frac{R e^{2 / 3}}{6} \epsilon^{-1.78}\right), \quad R e<1000 \\
C_{d}=0.424, \quad R e>1000 \\
C_{d}=C_{d}, \quad M<0.3 \\
C_{d}=C_{d}+\frac{1-0.47}{1.5-0.3}(M-0.3), \quad 0.3<M<1.5 \\
C_{d}=C_{d}+\frac{0.93-1}{6.0-1.5}(M-1.5), \quad 1.5<M<6.0 \\
C_{d}=C_{d}+0.46, \quad M>6.0
\end{gathered}
$$

The Reynolds number, Re, is defined as;

$$
R e=\frac{2 \rho_{g}\left|\mathbf{v}_{\mathbf{g}}-\mathbf{v}_{\mathbf{p}}\right| r_{p}}{\mu_{g}}
$$

In equation $2.17, \mu_{g}$ is the viscosity of the gas. The viscosity is considered to be a function of temperature following the Sutherland's law given in equation 2.18, where $T_{r e f}$ is the reference temperature, $\mu_{r e f}$ is the reference viscosity at $T_{r e f}$, and $S$ is the Sutherland temperature. 


$$
\mu_{g}=\mu_{\text {ref }}\left(\frac{T}{T_{\text {ref }}}\right)^{3 / 2} \frac{T_{r e f}+S}{T+S}
$$

The particle volume fraction, $\theta$, is related to the distribution function, $h$, and the mass of the particle, $m_{p}$, and is given by equation 2.19 .

$$
\theta=\iint h \frac{m_{p}}{\rho_{p}} d m_{p} d \mathbf{v}_{\mathbf{p}}
$$

The sum of the volume fraction of particles and the voidage fraction of the of gas is unity.

$$
\theta+\epsilon=1
$$

Now, the inter-phase momentum transfer term, $F_{s}$, which is the particle source term for momentum is given by equation 2.21 .

$$
F_{s}=\iint h m_{p}\left[D_{p}\left(\mathbf{v}_{\mathbf{g}}-\mathbf{v}_{\mathbf{p}}\right)-\frac{1}{\rho_{p}} \nabla P\right] d m_{p} d \mathbf{v}_{\mathbf{p}}
$$

The particle energy equation is based on the balance of energy transfer from the particle due to convective heat transfer, evaporation and radiative heat transfer, with the change in the internal energy of the droplet as given by the Ranz-Marshall correlation [56].

$$
m_{p} C_{p, l} \frac{d T_{p}}{d t}=2 \pi r_{p} K_{g} N u\left(T_{g}-T_{p}\right)+\frac{d m_{p}}{d t} H_{f g}+\dot{Q_{H}}
$$

In equation $2.22, C_{p, l}$ is the specific heat of the liquid, $K_{g}$ is the conductivity of the gas, $T_{g}$ is the temperature of the gas, $T_{p}$ is the temperature of the particles, $N u$ is the Nusselts number, and $H_{f g}$ is the latent heat of vaporization of the particles and $\dot{Q_{H}}$ is the rate heat transfer due to radiation which is neglected in our study. O'rourke [55] gave the correlation for the void fraction dependence of the Nusselts number. The equation, after eliminating the effect of the vapor transfer function on 
energy transport, is given by equation 2.23, where $\operatorname{Pr}$ is the Prandtl's number which is described in equation 2.24. In this equation $C_{p}$ is the specific heat of the gas.

$$
\begin{gathered}
N u_{g}=\left[2 \epsilon^{-1.75}+0.6 \frac{R e^{1 / 2}}{\epsilon} \operatorname{Pr}^{1 / 3}\right] \\
\operatorname{Pr}=\frac{C_{p} \mu_{g}}{K_{g}}
\end{gathered}
$$

The change in particle mass, $d m_{p}$, is given by Crowe et al. [57]. The equation is derived by considering the mass transfer from the surface of the evaporating particle due to the diffusion of the vapor. This diffusion is driven by the difference in vapor concentration at the surface of the particle and the free stream. The resulting equation is given in equation 2.25, where $S h$ is the Sherwood number, $D_{v}$ is the diffusion constant, $w_{v s, \infty}$ is the vapor concentration in the free stream, and $w_{v s, s}$ is the vapor concentration at the surface of the particle given by equation 2.26 .

$$
\begin{gathered}
\frac{d m_{p}}{d t}=2 S h \pi r_{p} \rho_{g} D_{v}\left(w_{v s, \infty}-w_{v s, s}\right) \\
w_{v s, s}=\frac{M_{v s} P_{v s}}{M_{g, m i x} P_{g}}
\end{gathered}
$$

In equation $2.26, P_{v s}$ is the saturation pressure of the particle at its temperature, $P_{g}$ is the pressure of the gas, $M_{g, m i x}$ is the molecular mass of the gas mixture, and $M_{v s}$ is the molecular mass of the vapor species. The correlation given by Gilliland [58] is used to calculate diffusion constant, $D_{v}$, given in equation 2.27 where $M_{g, m i x}$ is the molecular mass of the mixture, $M_{v s}$ is the molecular mass of the vapor species, and $V_{1}$ and $V_{2}$ are the atomic diffusion volume of the gas species and vapor species respectively. 


$$
D_{v}=\frac{435.7 T_{p}^{3 / 2}}{P_{g}\left(V_{1}^{1 / 3}+V_{2}^{1 / 3}\right)^{2}} \sqrt{\frac{1}{M_{g, \text { mix }}}+\frac{1}{M_{v s}}}
$$

The Sherwood number in mass transfer is analogous to the Nusselt number for the non-evaporation energy transfer and is given by equation 2.28 where $S c$ is the Schmidt number given by equation 2.29 .

$$
\begin{gathered}
S h=2+0.6 R e^{1 / 2} S c^{1 / 3} \\
S c=\frac{\nu_{g}}{D_{v}}
\end{gathered}
$$

$\nu_{g}$ is the kinematic viscosity of the gas.

The saturation pressure, $P_{v s}$, of the particle at its temperature is predicted by using the Antoine equation (equation 2.30). By combining this equation with the Clausius-Clapeyron equation, The value of the latent heat of vaporization, $H_{f g}$, is calculated using equation 2.31 .

$$
\begin{gathered}
\log \left(P_{v s}\right)=A-\frac{B}{C+T_{p}} \\
H_{f g}=\frac{T_{p}^{2} R B \log (10)}{C+T_{p}}
\end{gathered}
$$

$\mathrm{A}, \mathrm{B}$, and $\mathrm{C}$ are the Antoine coefficients. In the model given, the particles are considered to be water and values for their Antoine coefficients are obtained from the National Institute of Standards and Technology (NIST) and are summarized in the Table 2.1.

For water particles, the use of the Antoine equation for calculating the saturation pressure is efficient as the Antoine constants are well researched for the temperature 


\begin{tabular}{|l|c|c|c|c|}
\hline Temperature $(\mathrm{K})$ & $\mathrm{A}$ & $\mathrm{B}$ & $\mathrm{C}$ & Reference \\
\hline $255.9-373$ & 4.6543 & 1435.264 & -64.848 & {$[59]$} \\
\hline $379-573$ & 3.55959 & 643.748 & -198.043 & {$[60]$} \\
\hline
\end{tabular}

Table 2.1: Antoine coefficients for water

range of interest. In this work, the author focuses on water particles, but this evaporation model can be used for other substances too in the range of availability of the Antoine coefficients. In Table 2.1, there is a small discontinuity between the temperature from $373 \mathrm{~K}$ to $379 \mathrm{~K}$. The Antoine coefficients in that range are considered to be equal to the low temperature values given by [59] (values in the first row of the table).

Now, the interphase energy transfer term, $E_{s}$, which is the particle source term for energy transfer is given by equation 2.32 .

$$
\begin{gathered}
E_{s}=\iint h m_{p}\left[D_{p}\left(\mathbf{v}_{\mathbf{g}}-\mathbf{v}_{\mathbf{p}}\right)^{2}-C_{p, l} \frac{d T_{p}}{d t}-C_{v s} \frac{d m_{p}}{d t}\right] d m_{p} d \mathbf{v}_{\mathbf{p}} \\
E_{s}=E_{d, \zeta}+E_{c v}+E_{e v}
\end{gathered}
$$

In equation $2.33, E_{d, \zeta}$ is the interphase energy transfer due to the drag heating given by expression $D_{p}\left(\mathbf{v}_{\mathbf{g}}-\mathbf{v}_{\mathbf{p}}\right)^{\mathbf{2}}, E_{c v}$ is due to the convective heat transfer given by $-C_{p, l} \frac{d T_{p}}{d t}, E_{e v}$ is due to evaporation given by $-C_{v, s} \frac{d m_{p}}{d t}$, and $C_{v s}$ is the specific heat of the vapor species at constant volume. In equation 2.22 , It can be seen that the rate of energy change of the particle during evaporation is $H_{f g} \frac{d m_{P}}{d t}$, but the amount of energy change of the the gas would be due to the internal energy carried by the evaporated vapor phase as given by $E_{e v}$. So, there is a loss of some amount of energy released due to difference in enthalpy of formation of vapor and water during droplet evaporation. 
The interphase mass transfer term, $M_{s}$, is the particle source term for mass transfer and is given in equation 2.34 .

$$
M_{s}=\iint h\left[\frac{d m_{p}}{d t}\right] d m_{p} d \mathbf{v}_{\mathbf{p}}
$$

These particle source terms are coupled to the gas conservation equations by interpolating them to the Eulerian grid using a suitable interpolation operator.

\subsubsection{Interpolation method}

The mapping of the particle properties to and from the grid to the particle location is done by interpolation. The interpolation is done by the locally and globally conservative trilinear interpolation operator given by Snider [25]. The operator can be used to map particle properties either to the center or to the faces of the zone. Since the model is used for the collocated grid, all the particle properties are mapped to the center of the zone except the particle volume fraction, $\theta$, which appears as a face variable on the grid in the discretized conservation equation and is interpolated to the face of the zone.

In the three dimensional case, the interpolation operator at the grid is given by the product of the directional operators in $X, Y$ and $Z$ coordinates. The interpolation operator for a particle, $p$, to the grid position $i, j, k$ is given by equation 2.35 .

$$
S_{i j k, p}=S_{i, p}^{x} S_{j, p}^{y} S_{k, p}^{z}
$$

The directional interpolation operator for the $X, Y$, and $Z$ coordinates is conservative in nature, varying linearly from 1 to 0 with 1 being at the location of the particle position and 0 at or greater than the distance of the grid unit dimension.

Let us suppose a particle is located at position $\mathbf{x}_{\mathbf{p}}$ where $\mathbf{x}_{\mathbf{p}}=x_{p} i+y_{p} j+z_{p} k$. The interpolation operator on the $X$ direction at the location of particle $x_{p}$ on grid 
coordinate $i$ is given by equation 2.36 .

$$
S_{i, p}^{x}=\frac{x_{i+1}-x_{p}}{x_{i+1}-x_{i}}
$$

Similarly, the interpolation operator $S_{j}{ }^{y}\left(y_{p}\right)$ and $S_{k}{ }^{z}\left(z_{p}\right)$ for the $Y$ and $Z$ coordinate systems can also be found. The gas property interpolated to the particle location denoted by the subscript $g p$ can be calculated as below.

$$
P_{g p}=\sum_{i j k} S_{i j k, p} P_{i j k}
$$

To map the properties at the faces of the grid, they were first interpolated to the center of the grid by the trilinear interpolation operation, and then equation 2.38 was used to find the value of the properties at the faces of the zone. This method is consistent with the face trilinear interpolation given by Snider [25].

$$
S_{i-\frac{1}{2}, j, k}=\frac{S_{i-1, j, k}+S_{i, j, k}}{2}
$$

\subsubsection{Momentum transfer}

The numerical method given by Andrews and O'rourke [20] is the basis for the momentum exchange numerical method given in this paper. However, the method has been significantly modified to make it simpler and more suitable for the shock driven case. The continuum representation of the particulate phase is not used in our method making it a PIC method as opposed to MP-PIC method. It is an Eulerian-Lagrangian method where the gas is defined in the Eulerian coordinate system and the particles are defined in the Lagrangian coordinate system. In this method, single particles are not solved as it is very inefficient and is computationally exhausting. A number of particles are grouped together to define a computational particle, called a parcel, for 
computation. While coupling the particles to the grid, the volume fraction effects of the particles must be considered. The particle volume fraction, $\theta$, is calculated by equation 2.39 .

$$
\theta_{i j k}=\frac{1}{V_{i j k}} \sum_{p} N_{p} \frac{m_{p}}{\rho_{p}} S_{i j k}\left(\zeta_{p}\right)
$$

In equation $2.39, V_{i j k}$ is the volume of the grid containing parcel, $V_{p}$ is the volume of the single particle, $N_{p}$ is the number of particles in a parcel, and $\zeta_{p}$ is the position of the particle in $\zeta$ direction. The interpolation operator is calculated by considering only the position of the particle in the $\zeta$ direction because of the one dimensional implementation of the PPM.

While solving for the Lagrangian equations, the gas properties that appear have to be interpolated from the Eulerian grid to the parcel location. The parcel changes its position within a time step which must be considered in the numerical method for accuracy. Such approximation is made by mapping the interpolated gas properties to the provisional parcel position given by equation 2.40 , where $\bar{\zeta}_{p}$ is the provisional parcel location.

$$
\bar{\zeta}_{p}=\zeta_{p}^{n}+u_{p}^{n} \Delta t
$$

The gas properties mapped to provisional particle location are approximated by the following equations;

$$
\begin{aligned}
& \rho_{g p}=\sum S_{i j k}\left(\bar{\zeta}_{p}\right) \rho_{g}^{n} \\
& u_{g p}=\sum S_{i j k}\left(\bar{\zeta}_{p}\right) u_{g}^{n}
\end{aligned}
$$




$$
\begin{gathered}
\bar{\theta}_{i j k}=\frac{1}{V_{i j k}} \sum_{p} N_{p} \frac{m_{p}}{\rho_{p}} S_{i j k}\left(\bar{\zeta}_{p}\right) \\
\bar{\epsilon}=1-\bar{\theta}
\end{gathered}
$$

The advanced time velocity of the particle is obtained by integration of equation 2.12 and is given by;

$$
u_{p}^{n+1}=\frac{u_{p}^{n}+\Delta t\left[D_{p} u_{g p}^{n+1}+g-\frac{1}{\rho_{p}}\left(\frac{\bar{P}_{i+1 / 2}-\bar{P}_{i-1 / 2}}{\Delta \zeta}\right)_{p}\right]}{1+\Delta t D_{p}}
$$

In equation 2.45, $i$ is the grid index in the $\zeta$ direction and $\bar{P}$ is the approximated advanced time pressure flux obtained at the face of the cell by Reimann's solution. The superscript $n$ and $n+1$ denotes the current time and advanced time properties respectively, and $\Delta t$ is the time step. Now, the source term for momentum exchange is given by;

$$
F_{\zeta}=\frac{1}{V_{i j k}}\left\{\sum_{p} S_{i j k}\left(\bar{\zeta}_{p}\right)\left[D_{p}\left(u_{g p}^{n+1}-u_{p}^{n+1}\right)-\frac{1}{\rho_{p}}\left(\frac{\bar{P}_{i+\frac{1}{2}}-\bar{P}_{i-\frac{1}{2}}}{\Delta \zeta}\right)_{p}\right] N_{p} m_{p}\right\}
$$

If the advanced time particle velocity term is replaced from the particle momentum source term, the equation is in the form as below;

$$
F_{\zeta}=A_{\zeta}^{\prime} u_{g p}^{n+1}-B_{\zeta}^{\prime}-C_{\zeta}^{\prime}\left(\frac{\bar{P}_{i+\frac{1}{2}}-\bar{P}_{i-\frac{1}{2}}}{\Delta \zeta}\right)_{p}
$$

The expressions for $A_{\zeta}^{\prime}, B_{\zeta}^{\prime}$ and $C_{\zeta}^{\prime}$ are given below.

$$
A_{\zeta}^{\prime}=\frac{1}{V_{i j k}} \sum_{p} S_{i j k}\left(\bar{\zeta}_{p}\right) \frac{D_{p}}{1+\Delta t D_{p}} N_{p} m_{p}
$$




$$
\begin{aligned}
B_{\zeta}^{\prime} & =\frac{1}{V_{i j k}} \sum_{p} S_{i j k}\left(\bar{\zeta}_{p}\right) \frac{D_{p}\left(u_{p}^{n}-\Delta t g\right)}{1+\Delta t D_{p}} N_{p} m_{p} \\
C_{\zeta}^{\prime} & =\frac{1}{V_{i j k}} \sum_{p} S_{i j k}\left(\bar{\zeta}_{p}\right) \frac{1}{\rho_{p}\left(1+\Delta t D_{p}\right)} N_{p} m_{p}
\end{aligned}
$$

The solution step involves solving the conservation equations on the grid. The conservation equations are solved by using the zone average advanced time fluxes $\bar{\rho}$, $\bar{u}$, and $\bar{P}$ obtained by solving the appropriate Riemann problems. More about the Riemann solution and calculation of the average properties is given by Fryxell et al. [49].

The discretized form of the conservation equations for momentum and mass are given below. The splitting mechanism adds the given one dimensional conservation equations to give a full 3-D solution. The total and species conservation of mass equations are calculated without the source term, $M_{s}$, and are updated later.

$$
\begin{gathered}
\bar{\epsilon} \rho_{i}^{n+1}=\epsilon \rho_{i}^{n}-\frac{\Delta t}{\Delta V_{i j k}}\left(\bar{\epsilon}_{i+\frac{1}{2}} A_{i+\frac{1}{2}} \bar{\rho}_{i+\frac{1}{2}} \bar{u}_{i+\frac{1}{2}}-\bar{\epsilon}_{i-\frac{1}{2}} A_{i-\frac{1}{2}} \bar{\rho}_{i-\frac{1}{2}} \bar{u}_{i-\frac{1}{2}}\right) \\
\bar{\epsilon} \rho_{i}^{n+1} u_{i}^{n+1}=\epsilon \rho_{i}^{n} u_{i}^{n}-\Delta t A_{\zeta}^{\prime} u_{i}^{n+1}+\Delta t B_{\zeta}^{\prime}-\frac{\Delta t}{\Delta V_{i j k}}\left(\bar{\epsilon}_{i+\frac{1}{2}} A_{i+\frac{1}{2}} \bar{\rho}_{i+\frac{1}{2}} \bar{u}_{i+\frac{1}{2}}^{2}\right. \\
\left.-\bar{\epsilon}_{i-\frac{1}{2}} A_{i-\frac{1}{2}} \bar{\rho}_{i-\frac{1}{2}} \bar{u}_{i-\frac{1}{2}}^{2}\right)-\frac{\Delta t}{\Delta \zeta}\left(\bar{P}_{i+\frac{1}{2}}-\bar{P}_{i-\frac{1}{2}}\right)\left(1-C_{\zeta}^{\prime}\right)+ \\
\frac{\Delta t}{2}\left(\epsilon \rho_{i}^{n} g_{i}^{n}+\bar{\epsilon} \rho_{i}^{n+1} g_{i}^{n+1}\right)
\end{gathered}
$$




$$
\begin{gathered}
\bar{\epsilon} \rho_{i}^{n+1} v_{i}^{n+1}=\epsilon \rho_{i}^{n} v_{i}^{n}- \\
\frac{\Delta t}{\Delta V_{i j k}}\left(A_{i+\frac{1}{2}} \bar{\epsilon}_{i+\frac{1}{2}} \bar{\rho}_{i+\frac{1}{2}} \bar{v}_{i+\frac{1}{2}} \bar{u}_{i+\frac{1}{2}}-A_{i-\frac{1}{2}} \bar{\epsilon}_{i-\frac{1}{2}} \bar{\rho}_{i-\frac{1}{2}} \bar{v}_{i-\frac{1}{2}} \bar{u}_{i-\frac{1}{2}}\right) \\
\bar{\epsilon} \rho_{i}^{n+1} w_{i}^{n+1}=\epsilon_{i}^{n} \rho_{i}^{n} w_{i}^{n}- \\
\frac{\Delta t}{\Delta V_{i j k}}\left(A_{i+\frac{1}{2}} \bar{\epsilon}_{i+\frac{1}{2}} \bar{\rho}_{i+\frac{1}{2}} \bar{w}_{i+\frac{1}{2}} \bar{u}_{i+\frac{1}{2}}-A_{i-\frac{1}{2}} \bar{\epsilon}_{i-\frac{1}{2}} \bar{\rho}_{i-\frac{1}{2}} \bar{w}_{i-\frac{1}{2}} \bar{u}_{i-\frac{1}{2}}\right) \\
\epsilon^{n+1} \rho_{i}^{n+1} X_{l i}^{n+1}=\epsilon^{n} \rho_{i}^{n} X_{l i}^{n}- \\
\frac{\Delta t}{\Delta V_{i}}\left(\bar{\epsilon}_{i+\frac{1}{2}} A_{i+\frac{1}{2}} \bar{\rho}_{i-\frac{1}{2}} \bar{X}_{l, i+\frac{1}{2}}-\bar{\epsilon}_{i+\frac{1}{2}} A_{i-\frac{1}{2}} \bar{\rho}_{i-\frac{1}{2}} \bar{X}_{l, i-\frac{1}{2}}\right)
\end{gathered}
$$

In equation $2.55, \bar{\epsilon}_{i+\frac{1}{2}}$ is calculated by $1-\bar{\theta}_{i+\frac{1}{2}}$, where $\bar{\theta}_{i+\frac{1}{2}}$ is calculated by equation 2.56 .

$$
\bar{\theta}_{i+1 / 2}=\frac{1}{V_{i j k}} \sum_{p} N_{p} V_{p} S_{i+\frac{1}{2} j k}\left(\bar{\zeta}_{p}\right)
$$

In the above discretized equations, the presence of advanced time properties does not make the solution implicit as those values are approximated by the Riemann solver and not solved implicitly. However, the particle source term for momentum is implicitly coupled to the conservation equations.

The splitting technique adds the solution for each dimension to give a 3-D solution. The details of this technique are given by Fryxell et al. [49]. Some scalar source terms are not calculated as directional fluxes, but are calculated as a value that repre- 
sents the complete change in a scalar variable on the grid due to the particles. Hence, if they are added to the directional conservation equation, their effect would be multiplied when each of the directional conservation equations are added by the splitting technique. To explain this, let us assume $\phi$ as the mass source term representing the amount of mass increase of the gas due to particle evaporation. Now, if $\phi$ is added to mass conservation equation in each direction then when those directional solutions are added to obtain the 3-D solution, the total mass increase in the grid would be overpredicted as $3 \phi$. So, the scalar source term is added only in the $X$ directional solution. Again, if it was added to the $Y$ or $Z$ directional solution, then it would not appear in the 1-D solution in $X$ direction.

\subsubsection{Energy transfer}

The numerical solution of the energy change of the non-evaporating particles is obtained by solving the discretized Ranz-Marshall correlation given by equation 2.22 in which the energy change due to mass transfer is neglected to be zero.

The discretized form of this non-evaporating energy transfer equation is obtained as equation 2.57 .

$$
\frac{d T_{p}}{d t}=\frac{F^{\prime} T_{g p}^{n+1}}{m_{p} C_{p, l}+\Delta t F^{\prime}}+\frac{m_{p} C_{p, l} T_{P}^{n}}{\Delta t\left(m_{p} C_{p, l}+\Delta t F^{\prime}\right)}-\frac{T_{p}^{n}}{\Delta t}
$$

or.

$$
\frac{d T_{p}}{d t}=G^{\prime} T_{g p}^{n+1}+H^{\prime}
$$

From the above equation, it can seen that the particle energy is implicitly solved. $F^{\prime}$ is a function given by;

$$
F^{\prime}=2 \pi r_{p} K_{g p} N u
$$

The source term for the energy transfer is given by equations 2.60 and 2.61 . 


$$
\begin{gathered}
E_{s_{1}}=\frac{1}{V_{i j k}}\left\{\sum_{p} S_{i j k}\left(\mathbf{x}_{\mathbf{p}}\right)\left[D_{p}\left(u_{p}-u_{g p}\right)^{2}-C_{p, l} \frac{d T_{p}}{d t}\right] N_{p} m_{p}\right\} \\
E_{s_{1}}=\frac{1}{V_{i j k}}\left\{\sum_{p} S_{i j k}\left(\mathbf{x}_{\mathbf{p}}\right)\left[D_{p}\left(u_{p}-u_{g p}\right)^{2}-C_{p, l}\left(G^{\prime} T_{g p}^{n+1}+H^{\prime}\right)\right] N_{p} m_{p}\right\}
\end{gathered}
$$

Here, the interpolation operators are computed by calculating the 3-D position vector of the particle. In the above equation $\mathbf{x}_{\mathbf{p}}$ is the position vector given by $x i+y j+z k$ and $E_{s_{1}}$ is given in the equation below.

$$
E_{s_{1}}=E_{d, \zeta}+E_{c v}
$$

$E_{d, \zeta}$ is the energy source due to the drag heating term, $D_{P}\left(u_{p}-u_{g p}\right)^{2}$, and $E_{c v}$ is the energy source term due to particle energy change by convection $-C_{p, l}\left(d T_{p} / d t\right)$. The drag heating is in terms of the velocity flux in the $\zeta$ direction, so it is directly updated to the energy conservation equation. However, the convection source term gives a complete change in the scalar variable $E$ of the gas due to the particle and hence should be only added in the $X$ direction as explained before.

$E_{c v}$ can be written as;

$$
\begin{gathered}
E_{c v}=-A_{T}^{\prime} T_{g p}^{n+1}-B_{T}^{\prime} \\
A_{T}^{\prime}=\frac{1}{V_{i j k}}\left\{\sum_{p} S_{i j k}\left(\mathbf{x}_{\mathbf{p}}\right)\left[C_{p, l} G^{\prime}\right] N_{p} m_{p}\right\} \\
B_{T}^{\prime}=\frac{1}{V_{i j k}}\left\{\sum_{p} S_{i j k}\left(\mathbf{x}_{\mathbf{p}}\right)\left[C_{p, l} H^{\prime}\right] N_{p} m_{p}\right\}
\end{gathered}
$$

The directional energy equation with the drag heating source term is given by equation 2.66 . 


$$
\begin{array}{r}
\bar{\epsilon} \rho_{i}^{n+1} E^{n+1}=\epsilon \rho^{n} E_{i}^{n}-\frac{\Delta t}{\Delta V_{i}}\left[A_{i+\frac{1}{2}}\left(\bar{\epsilon}_{i+\frac{1}{2}} \bar{\rho}_{i+\frac{1}{2}} \bar{E}_{i+\frac{1}{2}}+\bar{P}_{i+\frac{1}{2}}\right) \bar{u}_{i+\frac{1}{2}}\right. \\
\left.-A_{i-\frac{1}{2}}\left(\bar{\epsilon}_{i-\frac{1}{2}} \bar{\rho}_{i-\frac{1}{2}} \bar{E}_{i-\frac{1}{2}}+\bar{P}_{i-\frac{1}{2}}\right) \bar{u}_{i-\frac{1}{2}}\right]-P(\bar{\epsilon}-\epsilon)+ \\
\frac{\Delta t}{2}\left(\epsilon \rho_{i}^{n} g_{i}^{n}+\bar{\epsilon} \rho_{i}^{n+1} g_{i}^{n+1}\right)+E_{d, \zeta}
\end{array}
$$

The convection source term $E_{c v}$ is updated only in $X$ direction by using the equation below.

$$
E_{i}^{n+1}=\frac{E_{i}^{n+1} \rho_{i}^{n+1} \bar{\epsilon}}{\rho_{i}^{n+1} \bar{\epsilon}+\frac{\Delta t A_{T}^{\prime}}{C_{v, \text { mix }}}}-\frac{\Delta t B_{T}^{\prime}}{\rho_{i}^{n+1} \bar{\epsilon}+\frac{\Delta t A_{T}^{\prime}}{C_{v, \text { mix }}}}
$$

Where $C_{v, \text { mix }}$ is the specific heat at constant volume of the gas mixture in the cell.

\subsubsection{Mass transfer by evaporation}

Mass transfer affects not only the phase mass but also modifies the energy equation. The energy equation for the evaporating particles is obtained by solving the RanzMarshall correlation given by equation 2.22. The mass transfer energy equation has an additional term accounting to the energy change by the evaporation.

$$
\frac{d T_{p}}{d t}=\left(\frac{d T_{p}}{d t}\right)_{c v}+\frac{d m_{p}}{d t} \frac{3 H_{f g}}{4 C_{l, p} \rho_{p} \pi r_{p}^{3}}
$$

Where, $\left(\frac{d T_{p}}{d t}\right)_{c v}$ is the energy change given by equation 2.57 and $H_{f g}$ is given by equation 2.31 .

The source terms for mass $M_{s}$ and energy $E_{e v}$ due to droplet evaporation are given as: 


$$
\begin{gathered}
M_{s}=\frac{1}{\Delta t V_{i j k}}\left\{\sum_{p} S_{i j k}\left(\mathbf{x}_{\mathbf{p}}\right) d m_{p} N_{p}\right\} \\
E_{e v}=-\frac{C_{v, v s}}{\Delta t V_{i j k}}\left\{\sum_{p} S_{i j k}\left(\mathbf{x}_{\mathbf{p}}\right) d m_{p} N_{p} T_{p}\right\}
\end{gathered}
$$

Since $M_{s}$ and $E_{e v}$ are also scalar variables, a similar procedure as was discussed before for updating the scalar variable only in $X$ direction is followed.

$$
\begin{gathered}
\bar{\epsilon} \rho_{i}^{n+1}=\bar{\epsilon} \rho_{i}^{n+1}-\Delta t M_{s} \\
\bar{\epsilon} X_{v s, i} \rho_{i}^{n+1}=\bar{\epsilon} X_{v s, i} \rho_{i}-\Delta t M_{s} \\
\bar{\epsilon} X_{l, i} \rho_{i}^{n+1}=\bar{\epsilon} X_{l, i} \rho_{i}, \quad, l \neq v s \\
E_{i}^{n+1}=E_{i}^{n+1}+\Delta t \frac{E_{e v}}{\rho_{i}^{n+1} \bar{\epsilon}+\frac{\Delta t A_{T}^{\prime}}{C_{V, m i x}}}
\end{gathered}
$$

The update of the mass for the gas phase is a three step process as shown in equations 2.71 through 2.73. First, the total mass of the gas is updated, and then the mass fraction of the vapor species and later the mass fraction of the remaining gas species is updated. After the mass is updated, the energy change in the gas is updated using equation 2.74 .

\subsubsection{Implementation in the FLASH code}

The numerical methods discussed in the previous sections were implemented in the hydrodynamics code FLASH developed by the FLASH center at the University of Chicago. FLASH is a multiphysics simulation code capable of handling compressible 
flow problems. It is a highly efficient solver which gives us the choice of PARAMESH or CHOMBO grid structures with adaptive mesh refinement (AMR). AMR is a refinement technique which provides high resolution at the region of interest defined by the user and low resolution elsewhere resulting in efficient computation. The FLASH code has a built in PPM hydro solver and the cloud in cell interpolation operator, which is a trilinear interpolation operator that can interpolate the particle properties to the center of the grid or the fluid properties to the particle location. However, it is not capable of mapping particle properties at the faces of the grid. In its current state, the FLASH particle unit consists of passive particles which can be used to track the flow and active particles which use the charged particle and sink particle implementation. The charged particles are used to model ions in simulations of plasma while treating the electrons as a massless fluid and the sink particles are used in simulations of mass accretion during gravitational collapse in astrophysical environments.

The basic numerical scheme used during split implementation of the PPM is explained in the previous section. Various physical models in FLASH are arranged in a separate directory forming a unit. A unit in FLASH does a specific computation. FLASH has grid unit responsible for making the domain and dividing it into grids, a hydro unit for hydrodynamic computation, a physics unit for various physical computations like diffusion and the equation of state, a particle unit that solves for the different types of particles, a driver unit for making the framework for the code, and an IO unit for handeling input and output of the data files. While implementing the particle models, various modifications were done to the PPM files in the hydro unit and a directory for active particle computation named hybrid was added to the particle unit. The basic numerical algorithm followed while implementing the particle models in FLASH is given in the Figure 2.1.

A total of about 25 subroutines were created for implementation of the particle models. Among them the routines that do the major computations in the models are 


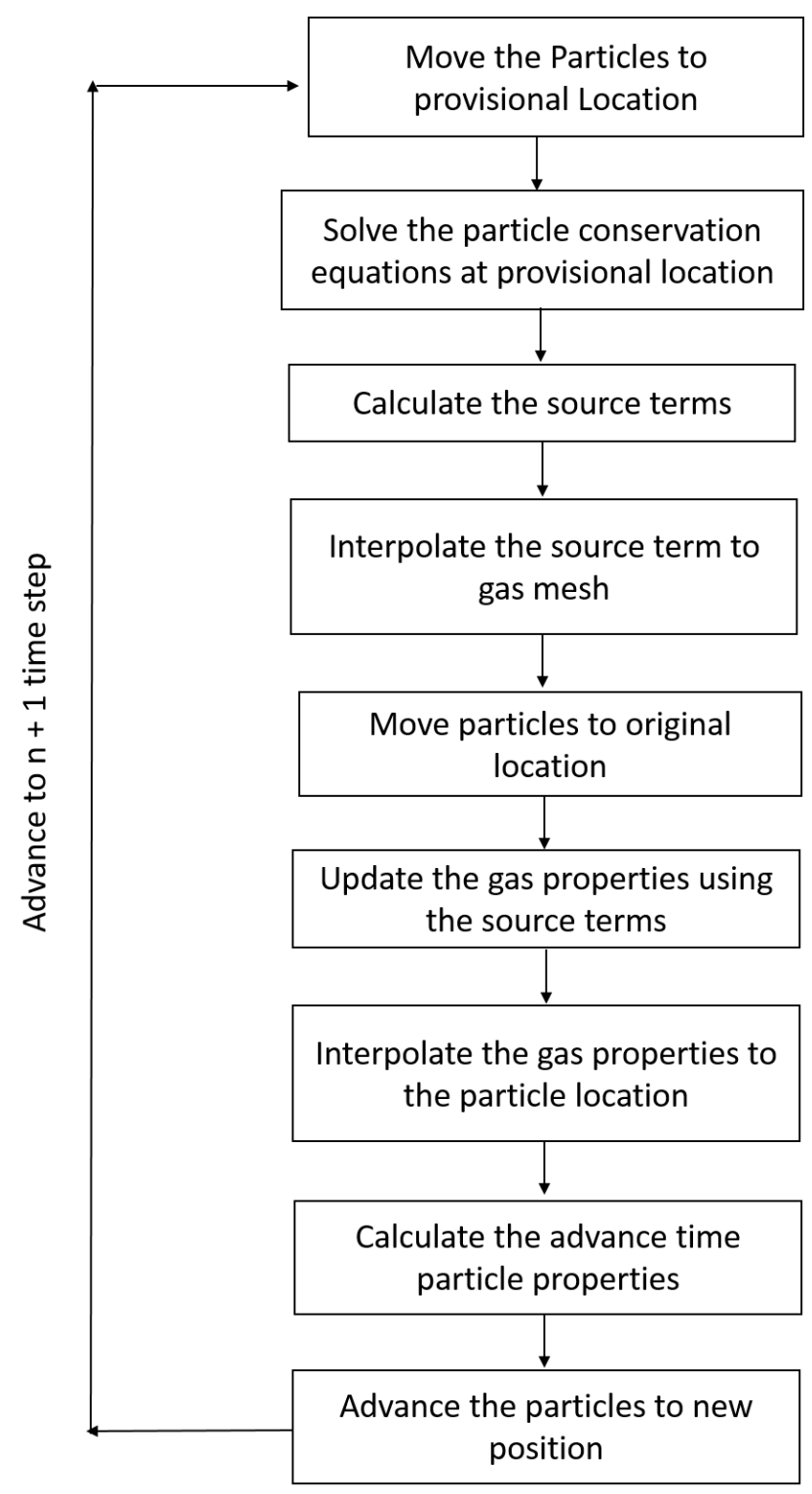

Figure 2.1: Numerical algorithm for the implementation of the particle models in FLASH 
described below.

- Hydro.F90: This is the master routine which controls the routines that calculate the source terms of the particles.

- pt_calculateVar.F90: The source terms for the particle momentum exchange is calculated here. First it advances the particles to provisional location and calculates the source terms. Then, It also interpolates those source terms to the gas variables. Last, the particles are advanced back to the original location.

- pt_calculateDrag. F90: This routine calculates the drag experienced by the particles in $\mathrm{X}, \mathrm{Y}$ and $\mathrm{Z}$ direction.

- pt_calculateVarTemp.F90: This routine calculates the energy and mass source terms. It solves the Ranz-Marshall correlation and calculates the energy source terms. FIrst, the particles are advanced to the provisional location and a check is performed to determine if there is mass transfer. If so, then it calculates the mass transfer by solving the Crowe's mass transfer model. It interpolates the source terms to the gas variables and then moves the particles back to the original location.

- pt_calculateTemp.F90: This routine solves for the mass transfer model and calculates the mass transfer source terms.

- hy_ppm_updateMom.F90: The modification of the gas conservation equations using the source terms interpolated to the gas variables is done here.

- pt_picAdvanceVel.F90: This routine calculates the new velocities of the particles using the updated gas properties. It also updates the position of the particles based on the new calculated velocity. 
- pt_picAdvanceCharged.F90: This is the master routine for updating properties of the particles. It makes the pt_picAdvanceVel call. In addition, it also updates the temperature of the particle and the new radius of the particle in the case of mass transfer. Furthermore, If the radius of the particles is zero, then the particles are deleted here.

The added particle models have a fully three dimensional implementation with particle momentum, energy, and mass transfer models where all of these models can be used independently or in combination with one another. It is integrated with the PPM hydro solver which uses the collocated grid structure. It is built in c.g.s units in the Cartesian coordinate system and the vapor phase of the evaporation model uses the multi-gamma equation of state. Care should be taken while using the evaporation model as the Antoine equations currently does not work below $273 \mathrm{~K}$ and above 573 K. In addition, we only have continuum mass transfer model. So, this model will break or give erroneous results if the particles radius is in the non-continuum regime. The complete code for each of the subroutine is attached in the Appendix.

\subsection{Validation}

In this section, validation of our models is done by comparing simulations with the analytical solutions and experiments. Single particle validation is shown for the momentum, energy, and mass transfer models. Further, a qualitative comparison of the momentum transfer model is shown with the experiment done by Vorobieff et al. [1].

\subsubsection{Particle temperature and velocity history}

The implementation of the particle momentum and energy equations in the FLASH code is validated with the analytical solution for the temperature and velocity history 


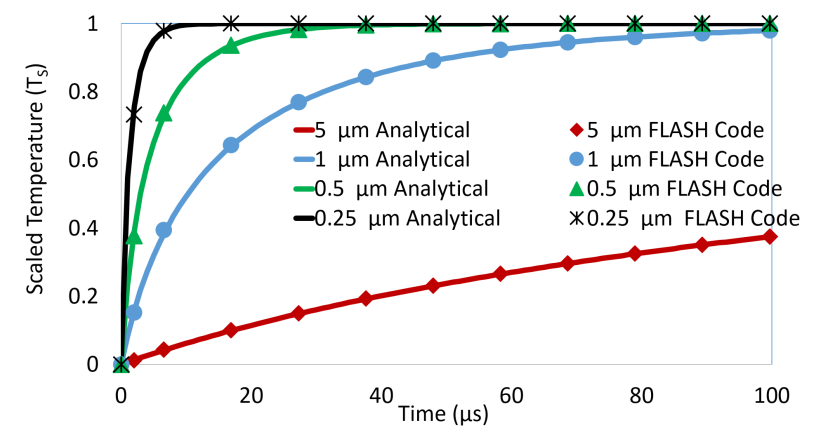

Figure 2.2: Single particle validation of the temperature history of the particle model in FLASH code with the analytical Solution. The line in the figure represents the analytical solution results and the markers represents the FLASH code results.

of a single particle as given by Cloutman [61]. The solution is obtained by considering the motion of a single particle in a fluid with constant fluid properties like density, temperature, velocity, and viscosity. In this comparison, a glycol particle was considered with density of $1.1 \mathrm{gm} / \mathrm{cm}^{3}$ injected into a flow field of nitrogen with a density of $0.0085 \mathrm{gm} / \mathrm{cm}^{3}$ and a temperature of $300 \mathrm{~K}$. The initial velocity, $u_{p}(0)$, and temperature of the particle, $T_{p}(0)$, is set to be $70000 \mathrm{~cm} / \mathrm{s}$ and $400 \mathrm{~K}$ respectively. Four different particle sizes, $0.25 \mu \mathrm{m}, 0.5 \mu \mathrm{m}, 1 \mu \mathrm{m}$, and $5 \mu \mathrm{m}$ radius, were used for the comparison. Cloutman [61] gave a high speed and low speed solution for a flow with $R e>1000$ and $R e<1000$ respectively. In our comparison case, the Reynolds number always meets the criteria for the low speed solution, $R e<1000$, so only the low speed solution is used for comparison. The solution is given by equations 2.75 and 2.76 , where the constants C, B, E, and F are given by Cloutman [61].

$$
\begin{gathered}
u_{p}(t)=\left\{\left(u_{p}(0)^{-2 / 3}+C\right) \exp \left[2 B\left(t-t_{o}\right) / 3\right]-C\right\} \\
T_{p}(t)-T_{g}=\left[T_{p}(0)-T_{g}\right] \exp \left\{-E\left(t-t_{o}\right)-E F\left[f\left(u_{p}(t)\right)-f\left(u_{p}(0)\right)\right]\right\}
\end{gathered}
$$




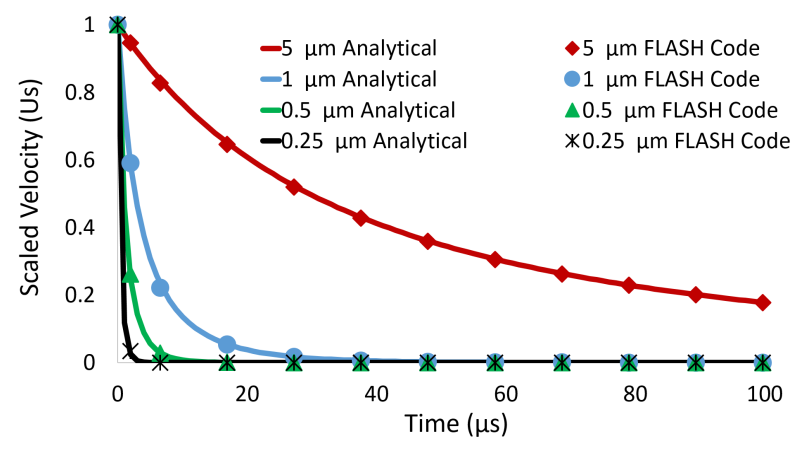

Figure 2.3: Single particle validation of the velocity history of the particle model in FLASH code with the analytical Solution. The line in the figure represents the analytical solution results and the markers represents the FLASH code results.

The results of the comparison are presented in Figures 2.2 and 2.3. In the Figures, the $Y$ axis is the variable scaled by its initial value at time zero. The scaled temperature is given as $T_{s}=T_{p}(t) / T_{p}(0)$ and scaled velocity as $U_{s}=u_{p}(t) / u_{p}(0)$ where $T_{p}(0)$ and $u_{p}(0)$ are the initial particle temperature and velocity respectively.

It can be seen that the temperature and velocity histories of the particle obtained from the simulation using our particle models in the FLASH code agree well at all times with that obtained from the analytical solution for all particle sizes. The analytical solution also uses the Kliatchko drag model, and since the effect of single particle on the fluid is negligible this agreement is expected for a correct implementation of our models in the code.

From the graph, it can be observed that the temperature and velocity lag increases as the diameter of particle increases. The higher lag is due to the larger value for the thermal relaxation time, $t_{T}$, and momentum relaxation time, $t_{v}$. The relaxation time represents the ability of the particles to equilibriate with the gas and is given by equations 2.77 and 2.78 .

$$
t_{v}=\frac{m_{p}}{6 \pi r_{p} \mu_{g p}}
$$




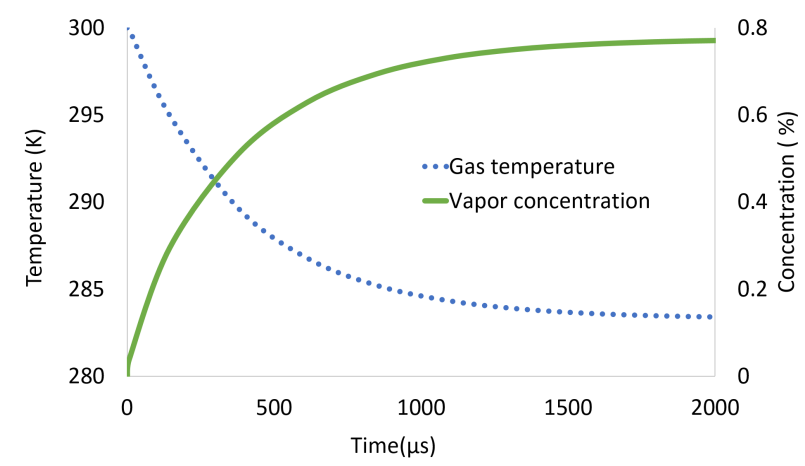

Figure 2.4: Time history of the gas temperature and vapor concentration in air due to particle evaporation. The primary axis is temperature $(\mathrm{K})$ and the secondary axis is concentration.

$$
t_{T}=\frac{m_{p} C_{p, l}}{4 \pi r_{p} K_{g p}}
$$

In these equations, it can seen that the thermal relaxation time is larger for the larger diameter particles. Again, the equilibrium temperature is lower for larger diameter particles due to their higher thermal mass.

\subsubsection{Particle evaporation properties}

To validate the evaporation model, a psychrometric analysis was done. A simple problem was set up with a $5 \mathrm{~cm}$ by $5 \mathrm{~cm}$ domain with resolution of $1 \mathrm{~cm}$ in the $X$ and $Y$ directions. Air was used as the carrier phase with initial temperature, $T_{g}$, set to be $300 K$, pressure, $P$, set to be $101.3 k P a$, and velocity, $u_{g}$, set to zero. A water parcel representing a large number of particles was initialized at the center of each cell. The initial temperature of the particles, $T_{p}$, was $300 \mathrm{~K}$, mass fraction was 3.7 $\%$, and the velocity, $u_{p}$, was zero. The particles were then allowed to evaporate to achieve psychrometric equilibrium, and the time history of the gas temperature and concentration of the evaporated vapor on the domain was plotted as shown in Figure 2.4 .

From Figure 2.4, it can see be seen that the saturation temperature of the gas 
is obtained to be about $283 K$ and the concentration of the water vapor in air at saturation is about $0.79 \%$. From the tabulated psychrometric properties of air, the amount of water vapor that dry air at $300 K$ can hold at saturation is about $7.5 \mathrm{gm} / \mathrm{Kg}$ of air, which gives the concentration of about 0.75 . Furthermore, the saturation temperature of dry air at $300 \mathrm{~K}$ is about $282 \mathrm{~K}$. These properties are very close to the results of the simulation which shows that the evaporation model given can accurately predict the mass transfer and temperature of the particles. The error in vapor concentration is due to the approximation of the latent heat of vaporization made by the Antoine equation.

\subsubsection{Comparison to experiments}

This section provides a further validation of the code as the results obtained from the simulations are qualitatively compared with experiments to identify if the model is prediciting the general characterisitics of the multiphase flow. The experimental results of Vorobieff et al. [1] are replicated with the help of simulations using the FLASH particle model. Further analysis of results was done to perdict the effect of particle distribution on the evolution of the particle interface.

Vorobieff et al. [1] performed experiments on the shock-driven instability of a particle seeded air cylinder surrounded by unseeded air. In this experiment, the two dimensional cylindrical column is formed by air-particle injection through a 6.35 $m m$ diameter annular nozzle. The experiment was done with a shock strength of $M$ $1.66 \pm 0.02$, which resulted in post shock velocity of about $303 \mathrm{~m} / \mathrm{s}$. The effective Atwood number is given to be $A \sim 0.03$ and was created by glycol droplets with diameters estimated to be in the submicron range to about $4 \mu \mathrm{m}$. The effective Atwood number is defined in equation 2.79, where subscript 1 and 2 indicate the 
upstream and the downstream gases respectively.

$$
A=\frac{\rho_{g p 2}-\rho_{g p 1}}{\rho_{g p 2}+\rho_{g p 1}}
$$

The experiment was approximated by running a 2D simulation with our particle model in the FLASH code. In order to simulate a shock tube environment, a domain of size $250 \mathrm{~cm}$ by $5 \mathrm{~cm}$ was set up. The left wall on the $X$ direction was setup to have an outflow boundary condition so as to maintaint the constant strength of the shock wave. All the other walls were made fully reflecting. The shock wave and interface were initialized at locations such that the interface was not affected by the reshock during our simulation time. In our simulation, the pressure, $P$, of the unshocked air is $101.325 \mathrm{KPa}$ and temperature, $T_{g}$, is $300 \mathrm{~K}$. The seeded air cylinder is shocked by a shock wave of strength $M=1.65$ resulting in a post shock velocity of $302 \mathrm{~m} / \mathrm{s}$. The mass fraction of the particles in the seeded air was $6.2 \%$ which gave $A \sim 0.03$. A section of the simulation domain with the air cylinder seeded with the particles and the shock wave is shown in Figure 2.5.

To make a valid comparison with the experiment, two different arrangements of particles were used, one with multiple size groups and one with a single size group. The multi-group arrangement has five different groups of particle sizes with group 1 containing the smallest radius particles and group 5 containing the largest radius particles. A log normal distribution of the particle radius was considered with a median diameter of $1 \mu \mathrm{m}$ and $96.7 \%$ of the particles being between $0.05 \mu \mathrm{m}$ and $4 \mu \mathrm{m}$ in diameter. The mean and standard deviation of the lognormal distribution were 0.35 and 0.74 respectively. Groups 1, 2, 3, and 4 contained approximately $23.1 \%$ of the total number of particles each, whereas group five, the largest radius, contained $4.5 \%$ of the particles. The median radius of each group was set as the representative diameter for the computation. Each zone was initialized with 17 computational particles, 


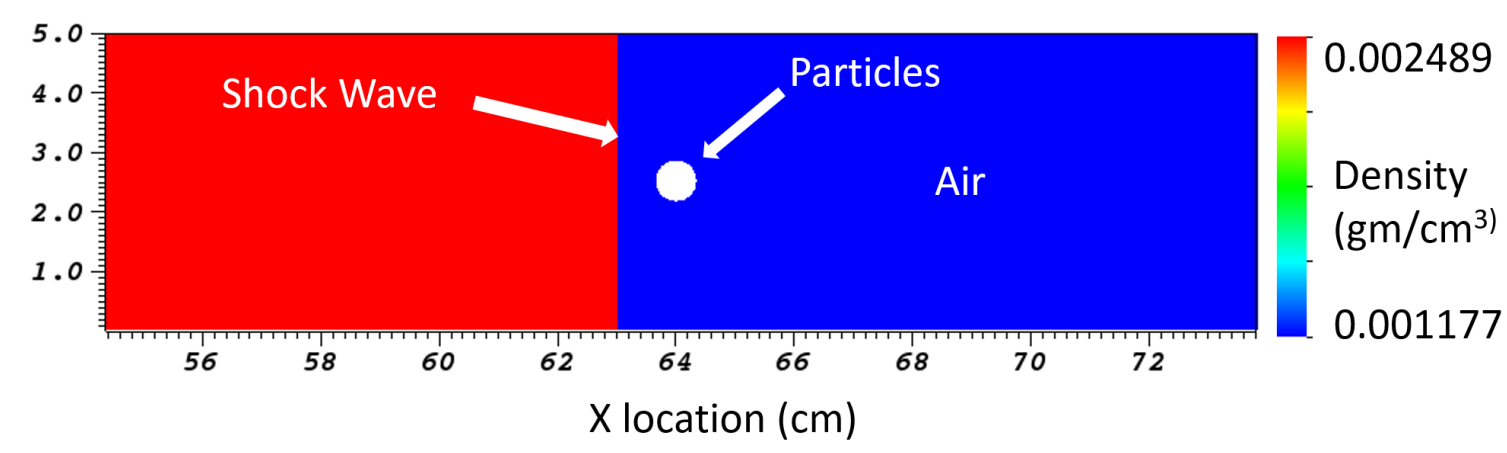

Figure 2.5: Initial condition for the simulation

four each for groups 1, 2, 3, and 4 which were distributed uniformly around the center of the zone. In this arrangement, in which four particles of each group are stacked on top of each other, and an additional single computational particle was added at the center of the zone for group 5. This created an approximately uniform distribution of particle parcel sizes among the groups. The distribution of the particles in a cell is shown in Figure 2.6. The single group arrangement was initialized with 17 particles with a $1 \mu m$ radius which are again distributed with 4 stacks of 4 particles arranged around the center of a zone and one particle at the zone center. The simulation was run at a resolution of $195 \mu \mathrm{m}$ or approximately 32 zones across the diameter of the circle. The representative radius and particle per parcel for each group is given in Table 2.2.

\begin{tabular}{|l|c|c|c|}
\hline Group & Radius $(\mu m)$ & Particles per parcel & $t_{v}(\mu s)$ \\
\hline 1 & 0.4 & 182 & 2.13 \\
\hline 2 & 0.9 & 167 & 10.8 \\
\hline 3 & 1.2 & 175 & 19.21 \\
\hline 4 & 1.6 & 173 & 34.15 \\
\hline 5 & 2.2 & 134 & 64.6 \\
\hline single group & 1.0 & 354 & 13.3 \\
\hline
\end{tabular}

Table 2.2: Radius, number of particles per parcel and momentum relaxation time for different groups

The results of the simulations together with the experiment is presented in Figure 

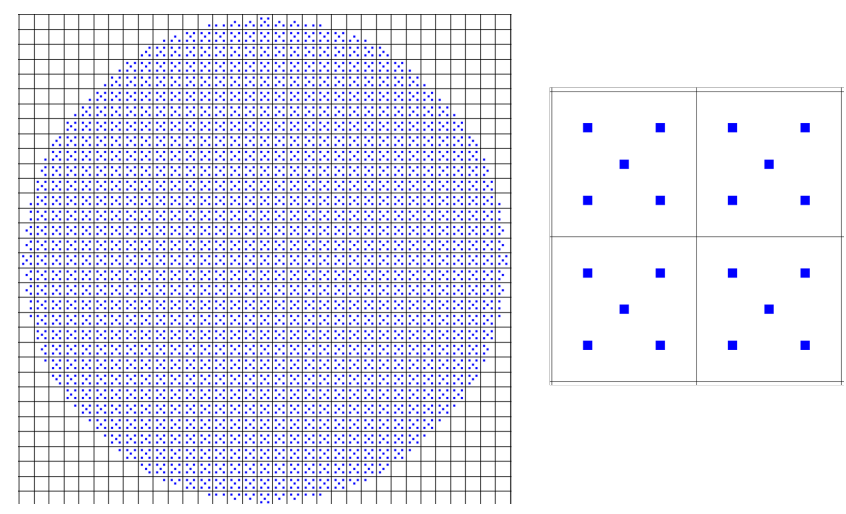

Figure 2.6: Left: Distribution of the particles in the air cylinder. Right: Four cells are magnified to show the arragement of particles in a cell
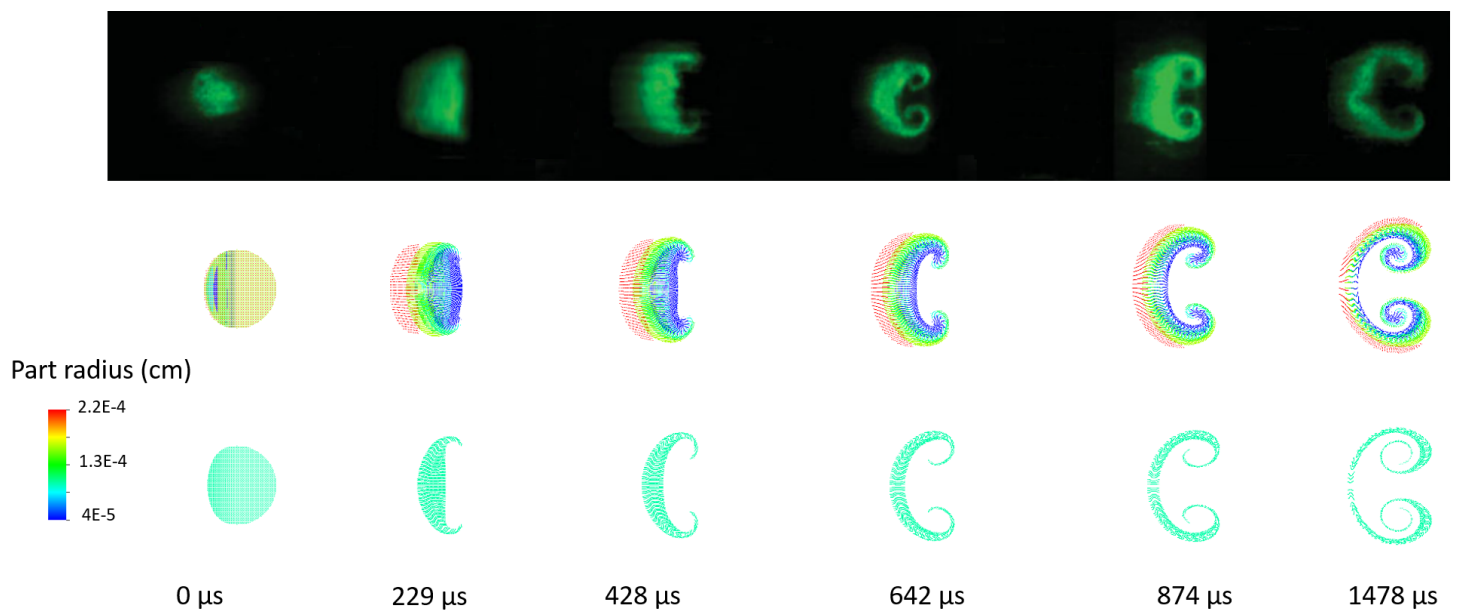

Figure 2.7: Comparison of of the FLASH simulation of the air cylinder with glycol particles of single group and multi-group with that of the Vorobieff experiments. The shock strength is $M=1.65$ and the average radius of the particle is $1 \mu \mathrm{m} . t=0$ corresponds to the time when the shock wave is at the center of the gas cylinder. Top: Vorobieff experimental results adapted with permission from [1], Copyrighted by the American Physical Society". Center: FLASH simulation of the multi-group of particles consisting of five different groups of radius $0.4 \mu \mathrm{m}, 0.9 \mu \mathrm{m}, 1.2 \mu \mathrm{m}, 1.6 \mu \mathrm{m}$ , $2.2 \mu \mathrm{m}$. Bottom: FLASH simulation with single group of particles of radius $1 \mu \mathrm{m}$. 
2.7. The development of the roll ups can be seen for the multi-group distribution which is very similar to that of the experiment. Drag causes the equilibrium velocity of the particles to be smaller than the gas velocity at all instances leading to the formation of roll-ups as a result of shear at the interface. Vorobieff et al. [1] suggests that this is not a RMI as there is no effective density difference between the seeded and unseeded gas, even though the evolution of the interface is similar to pure RM instability. By using 5 different groups of particles that follow a log normal distribution, an approximation of the distribution of particles by a typical particle producing device is made. However, no information of the median diameter of particles is available for the experiment, so the quantitative comparison between the two may show some discrepancies. The distribution of the particles at all times after the shock interaction shows the gradual increase in particle radius from the innermost region to the outer region of the interface. This is because the larger sized particles take more time to come to equilibrium due to their high velocity relaxation time and lag behind the flow. The simulation with the single particle distribution shows a more evolved but thinner interface. Having the same relaxation time for all particles results in all of them coming to equilibrium at the same time resulting in thinner interface.

The relaxation time calculated by considering a constant viscosity of 0.0001832 $c P$, given by Sutherland's law for air at $300 K$, is shown in the Table 2.2. Based on a qualitative comparison of the simulation and experimental results it can be seen that the particle size distribution has an important effect on the evolution of the interface and that replicating this distribution in simulations results in a result more similar to the experiment. The evolution of the simulation interfaces can be compared quantitatively by looking at the positive component of circulation, $\Gamma$, over time. Circulation gives an integrated measure of vorticity and is defined in equation 2.80 , where $d s$ is the differential area and $\omega$ is the vorticity. The positive component of circulation is defined in equation 2.81 where $\omega^{+}$represents only the positive vorticity. 


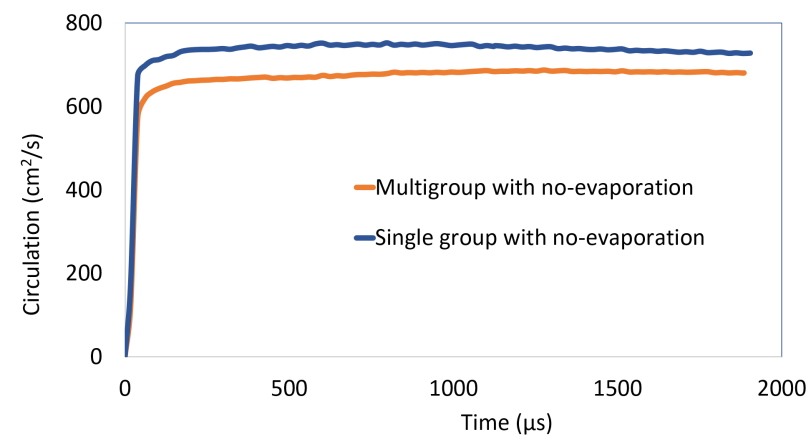

Figure 2.8: Circulation plot of the single group and multi-group particles without evaporation

$$
\begin{gathered}
\Gamma=\int \omega d s \\
\Gamma^{+}=\int \omega^{+} d s
\end{gathered}
$$

The circulation plot given in Figure 2.8 shows about an $11 \%$ drop in circulation for the multi-group distribution compared to the single group distribution. McFarland et al. [45] presents results that show that larger radius particles decrease the circulation. The author concludes that the advection of the particle vorticity source term relative to the flow reduces the effective circulation deposited for the large particles, whereas for the the small particles, this effect is negligible. The reduction of vorticity due to the larger particles in the multi-group simulation causes an overall decrease in the circulation of the interface. The author investigated the reason behind this decrease in circulation for larger particles by performing a quantitative analysis of a single particle in shock driven conditions in the next chapter. 


\section{Chapter 3}

\section{Simulation study in 2D}

In this chapter various multiphase parameters and their effect in the evolution of hydrodynamic instability are studied for a 2D circular interface. The particle model implemented in FLASH code is used for all the simulations. The two main parameters studied are the particle size distribution and the evaporation effects. The analysis procedure and the results are explained in the following sections.

\subsection{Single particle enstrophy}

To explain the reason behind the reduction in circulation by larger particles quantitatively, the author studied the vorticity production by two different particle groups with particle radii of $1 \mu m$ and $5 \mu m$ and with the parcel size set such that each particle group had the same mass. This will result in each group containing the same amount of momentum relative to the gas flow but having different relaxation times. The grid in this case is fully refined so as to eliminate the effect of the AMR. The simulation was set up similar to that of the experimental validation case taking a single particle as the simplest form of the particle-gas interface. The shock strength 


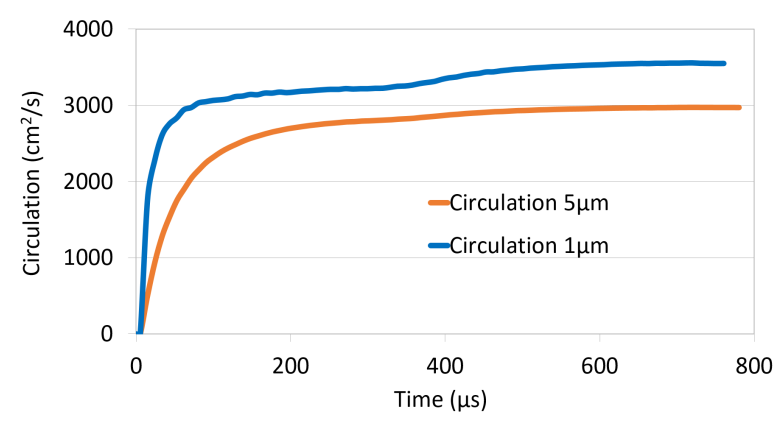

Figure 3.1: Circulation plot for $1 \mu m$ and $5 \mu m$ single particle

in this case is Mach 1.5 at a grid resolution of 64 nodes of size $780 \mu \mathrm{m}$ in $Y$ direction.

From Figure 3.1 it can be seen that there is less circulation for the $5 \mu \mathrm{m}$ particle than the $1 \mu \mathrm{m}$ particle as in the 2D circular interface case of the previous section. The vorticity equation has positive and negative components and only the positive component of the vorticity is summed to calculate the positive circulation. The effect of the vorticity production and diffusion terms in the total circulation is very difficult to study quantitatively as the positive component does not commute through the equation terms. Instead, vortical kinetic energy, known as Enstrophy, $\Omega(\omega)$, defined in equation 3.1, and the enstrophy production and dissipation terms are studied to understand this difference in circulation.

$$
\Omega(\omega)=\int \frac{\omega^{2}}{2} d s
$$

The enstrophy transport equation is shown in equation 3.2.

$$
\begin{aligned}
d_{t} \frac{\omega_{i} \omega_{i}}{2}+u_{g, j} \partial_{j} \frac{\omega_{i} \omega_{i}}{2} & =\omega_{i} \frac{1}{\rho_{g}} \varepsilon_{i j k} \partial_{j} \rho_{g} \partial_{k} P+\omega_{i} \omega_{j} \partial_{j} u_{g, i}-\omega_{i} \omega_{i} \partial_{j} u_{g, j} \\
& +\nu \partial_{j} \partial_{j} \frac{\omega_{i} \omega_{i}}{2}-\nu \partial_{j} \omega_{i} \partial_{j} \omega_{i}+\frac{1}{\rho_{g} \bar{\epsilon}} \omega_{i} \varepsilon_{i j k} \partial_{j} F_{k} \\
& =\dot{\Omega}_{b}+\dot{\Omega}_{s}+\dot{\Omega}_{t}+\dot{\Omega}_{f}+\dot{\Omega}_{d}+\dot{\Omega}_{r}
\end{aligned}
$$




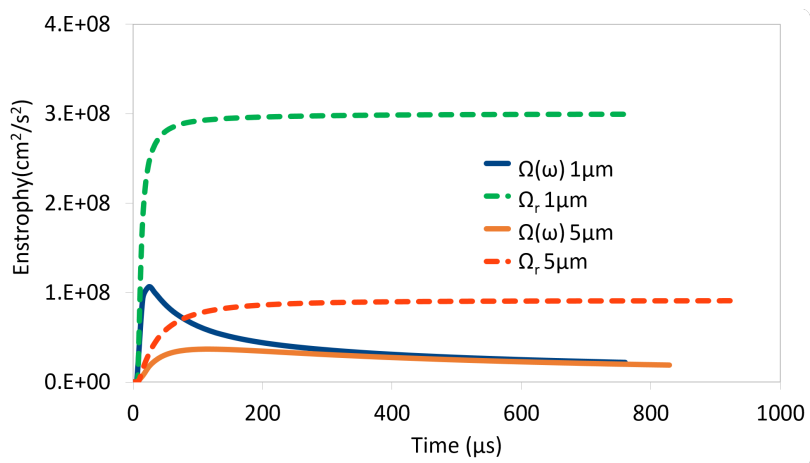

Figure 3.2: Enstrophy plot for $1 \mu \mathrm{m}$ and $5 \mu \mathrm{m}$ single particles. The solid line gives the actual enstrophy on the gas and the dotted line shows the enstrophy deposited by the source term

Here, $\dot{\Omega}_{b}$ is the baroclinic term of enstrophy, $\dot{\Omega}_{s}$ and $\dot{\Omega}_{t}$ are the enstrophy transport terms due to the stretching and dilatation, $\dot{\Omega}_{f}$ is the viscous diffusion term, $\dot{\Omega}_{d}$ is the viscous dissipation term, and $\dot{\Omega}_{r}$ is the production of the enstrophy by the particle momentum source term. The variables $F_{x}$ and $F_{y}$ in the source term, $\dot{\Omega}_{r}$, are given by equation 2.46. The production of enstrophy in the particle driven instability is only due to the source term as the baroclinic component is very small unlike the classical RMI.

Figure 3.2 shows the sum of enstrophy in each case over time and the cumulative sum of the enstrophy source term over all previous times. At early times, the enstrophy due to $1 \mu \mathrm{m}$ particles is larger in comparison to that of the $5 \mu \mathrm{m}$ particles. The vorticity produced by the source term is proportional to $\left(u_{g}-u_{p}\right) / r_{p}^{2}$ for particle groups with the same mass, as given by equation 2.46 . At very early times, the velocity deficit term, $u_{g}-u_{p}$, for both the particle sizes will be the same, so, the vorticity just depends on $1 / r_{p}^{2}$ giving a higher vorticity and hence the higher enstrophy for the smaller particles. At late times, the vorticity production is dominated by the velocity deficit term. The larger particles have a higher velocity difference due to the momentum lag, but still the velocity deficit term is small in magnitude when compared to its value at early times. So, it is expected that the vorticity source term for the particles 


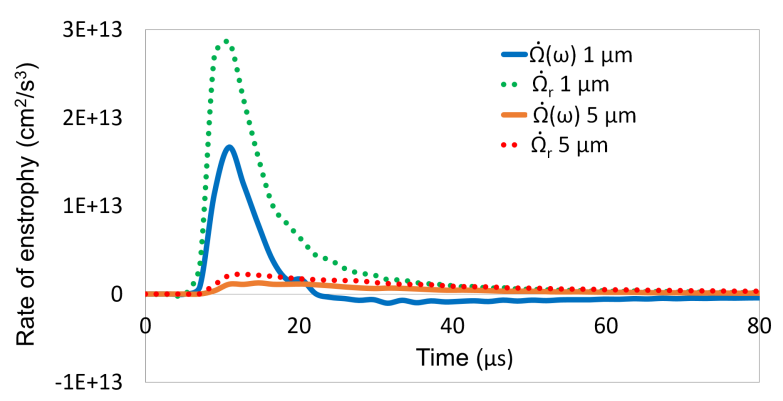

Figure 3.3: Time rate of enstrophy for $1 \mu \mathrm{m}$ and $5 \mu \mathrm{m}$ particle. Solid line gives the actual rate of enstrophy deposition and the dotted line shows the rate of enstrophy deposition by source term

increases at a very small rate at late times with the rate being slightly higher for the larger particles as seen in Figure 3.2.

Figure 3.3 shows the time rate of change of the enstrophy and the enstrophy source term. The solid line is the time rate of change of enstrophy in the grid which is maximum at early times when the shock wave reaches the particle position. The rate of enstrophy production by the source term is given by the dotted line. The enstrophy source term depends on both the strength of the vorticity source term and the strength of the underlying vorticity, or rather the alignment of these two vector fields. Since the source term advects through the gas flow field for the larger particles the vorticity field and source term are not well aligned until late times when the source term has weakened. This results in an overall smaller enstrophy source term for the $5 \mu m$ particles.

It can be seen in Figure 3.3 that the rate of production follows a similar trend as the rate of enstrophy change. Of all the terms in the enstrophy equation only the source term and the dissipation term are significant with the other terms being many orders of magnitude smaller. With this in mind the enstrophy dissipation term as the difference between the time rate of change of enstrophy and the enstrophy source term can be estimated. As the $5 \mu \mathrm{m}$ particles advect through the gas flow field they 


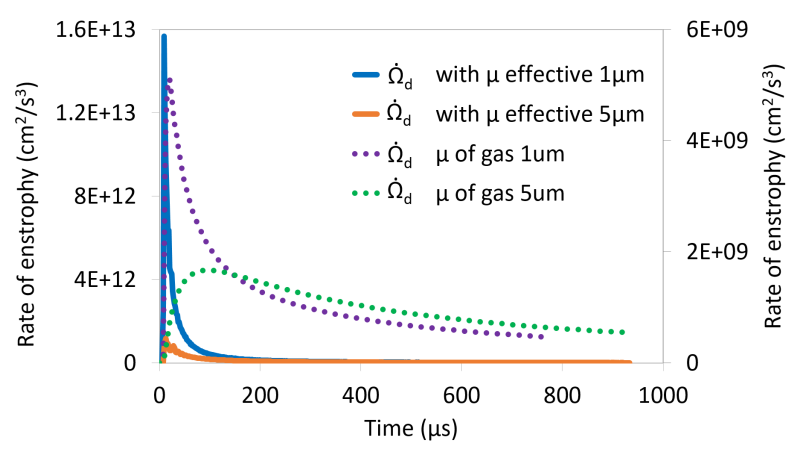

Figure 3.4: Rate of enstrophy dissipation for $5 \mu \mathrm{m}$ and $1 \mu \mathrm{m}$ particle. The solid line is the actual enstrophy dissipation plotted in the primary $Y$ axis and the dotted line is the enstrophy dissipation considering the viscosity of the gas plotted on secondary $Y$ axis

create a more distributed vortex field than the $1 \mu \mathrm{m}$ particles. This leads to the large particles having a lower gradient of vorticity and a lower dissipation at early and intermediate times. At late times the vorticity field has weakened in both cases and the dissipation becomes similar for both cases.

Direct calculation of the enstrophy dissipation term requires knowledge of the effective viscosity of the Eulerian grid. Since Euler equations are used the effective viscosity is due entirely to numerical effects making it difficult to calculate a priori. Instead the term can be estimated initially using a constant gas viscosity taken at a physical value $0.0001832 c P$. Figure 3.4 shows the enstrophy dissipation, (solid line), and estimate based on a constant viscosity, (dotted line). For the $1 \mu m$ particles the peaks of the actual enstrophy dissipation and the estimate occur at the same time and have similar trends. However, for the $5 \mu \mathrm{m}$ particles the peaks do not align as well with each other because the effective viscosity is modified by the multiphase effects which are highest for particles with high relaxation times. Despite the large difference in the dissipation values for the $5 \mu \mathrm{m}$ particle case the trends are very similar for both cases leading us to believe that the estimated enstrophy dissipation term can be used to gain insight into the mechanisms of multiphase enstrophy dissipation.

The enstrophy dissipation and estimate can be used to calculate an effective vis- 


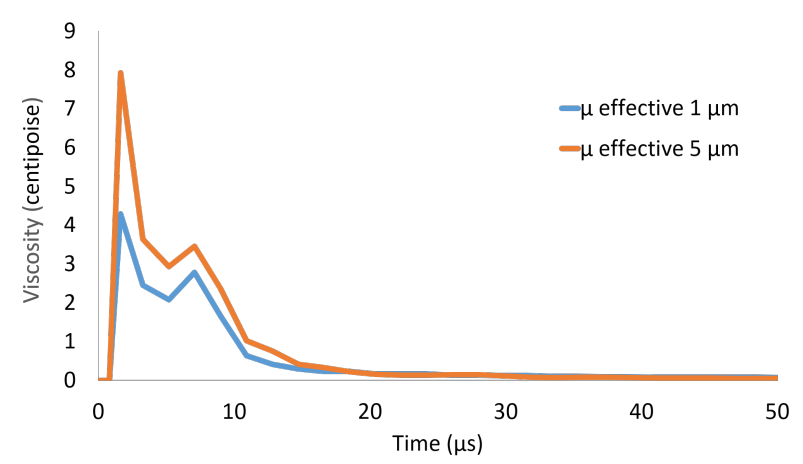

Figure 3.5: Effective viscosity for the $1 \mu \mathrm{m}$ and $5 \mu \mathrm{m}$ particle size

cosity for the simulation. This effective viscosity is given by Figure 3.5. It can be seen that at early times the effective viscosity for the $5 \mu \mathrm{m}$ particle size is much larger than the $1 \mu m$ particle size which causes the dissipation at early time to be much larger. At early times then the constant viscosity is a poor estimate of the effective viscosity. At late time, the effective viscosity is the same for both the particle sizes. It remains to be seen what part of the large change in effective viscosity is due to numerical effects and what part is due to physical multiphase effects. Future experimental work could help answer this question.

The previous plots are a simple one dimensional representation of an interface to simplify and better explain the problem, however these results will still hold for two and three dimensional cases, for example a larger number of computational particles which are spatially distributed. The physics will be similar with a small effect from the viscous diffusion term which will cause the vorticity deposited by particles to compete with each other. However, the viscous diffusion term is smaller by an order of magnitude than the actual vorticity deposited by the source term. 2D simulations with a higher effective resolution were run by using a uniform spatial distribution of particles with the same total mass as the single particle and same mass fraction. A higher circulation was obtained with increasing resolution but the same trend in enstrophy was found for the two particle sizes as was found in the single particle case. The higher circulation with increased resolution was explained by McFarland et al. 
[45] as the finer grid increased the ability of the simulation to capture smaller scale vortical features in the simulation.

\subsection{Simulation with evaporating particles}

In this section, the qualitative and quantitative study of the single group and multi group cases is done allowing the particles to evaporate.

\subsubsection{Qualitative effects}

The single group and multi-group particle distributions discussed in section 2.2.3 were run with the particle evaporation model to examine the effect of phase change on the evolution of the instability. Water droplets with a density $\rho_{p}=1 \mathrm{gm} / \mathrm{cc}$ and specific heat capacity $C_{p, l}=4.2 \mathrm{KJ} / \mathrm{Kg}-\mathrm{K}$ were initialized at $T_{p}=300 \mathrm{~K}$. A vapor phase was initialized in the gas phase so that the particles were initially in psychrometric equilibrium. To do this, the domain was considered to be a mixture of nitrogen and the equilibrium vapor concentration for water at $300 \mathrm{~K}$ which is calculated to be 2.16 \%. This prevented the particles from evaporating before the arrival of the shock front which would modify the effective Atwood number through evaporative cooling. The Table 3.1 gives the number of particles per parcel for each group of particles for each case to create the effective Atwood number of 0.03 for the evaporating particles.

The time evolution of the interface for the single group and multi-group particle distributions is given in Figure 3.6. This plot shows the particle radius plotted as discrete points on top of the continuous vapor phase. It can be seen that the multigroup particles evaporate much slower than the single group due to the presence of the larger particles. The multi-group simulation still has some unevaporated particles at $t=3483 \mu \mathrm{s}$ while for the single group all particles have evaporated before $t=2983$ $\mu s$. In addition, similar to the simulation without evaporation, the interface of the 


\begin{tabular}{|l|c|c|}
\hline Group & Radius $(\mu m)$ & Particles per parcel \\
\hline 1 & 0.4 & 198 \\
\hline 2 & 0.9 & 182 \\
\hline 3 & 1.2 & 190 \\
\hline 4 & 1.6 & 189 \\
\hline 5 & 2.2 & 146 \\
\hline single group & 1.0 & 385 \\
\hline
\end{tabular}

Table 3.1: Radius and number of particles per parcel of different groups for evaporating particles

single group case is more evolved than the multi-group case. The late time results show a perturbed interface on the downstream side for both cases. The particles at the edges of the rollups are the last to evaporate as seen in the multi-group interface at $t=2983 \mu \mathrm{s}$ and single group evaporation at $t=2212 \mu \mathrm{s}$. The particle lag is sufficient to prevent these particle from following the path of the roll-ups. As a result, those particles stray away from the interface in the direction of the flow giving rise to the perturbed edges. For the single group particles the jet like features ejected from the core in the down stream direction similar to the features documented by Anderson et al. [42] is seen. For the multi-group case the larger particles migrate to the outside edges and delay the development of these spike like features.

\subsubsection{Quantitative effects}

The circulation plot given in Figure 3.7 shows the increase in circulation for the evaporation case in comparison to the non-evaporating case. The increase in circulation is due to the density gradient created as a result of the evaporative cooling of the interface. This density gradient can produce secondary baroclinic vorticity deposition as a result of the centripetal acceleration, but also strengthens the particle vorticity source term through the gradient of the drag force (equation 2.13). The evaporating single group has a higher circulation than the evaporating multi-group case for the 


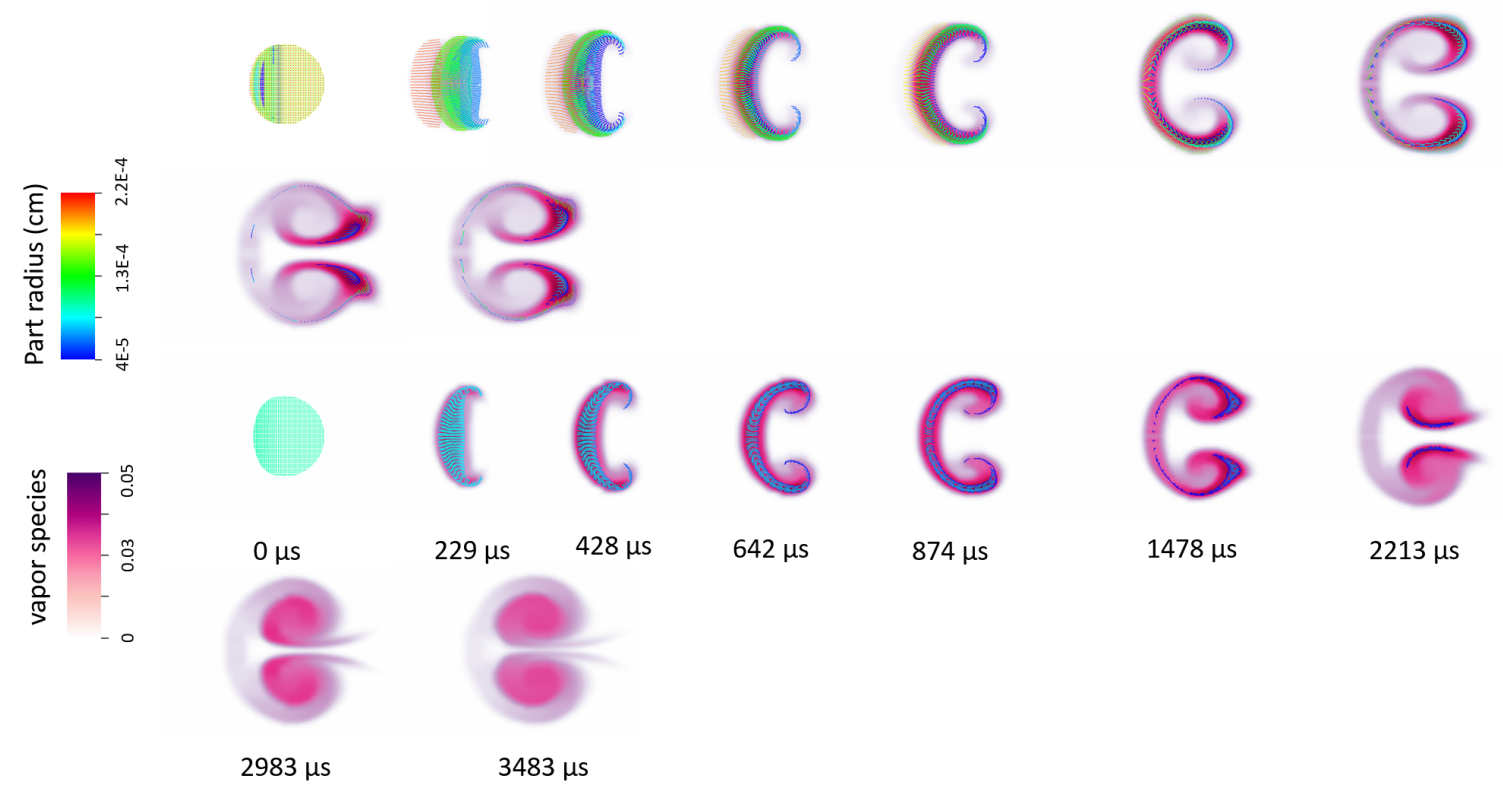

Figure 3.6: Plot of FLASH simulation of the single group and multi-group of particles at $M=1.65$ with evaporation. The particles are plotted on top of evaporated vapor species with different color bars. Top: evaporating multi-group of particles with radius $0.4 \mu \mathrm{m}, 0.9 \mu \mathrm{m}, 1.2 \mu \mathrm{m}, 1.6 \mu \mathrm{m}, 2.2 \mu \mathrm{m}$. Bottom: evaporating single group of particles with radius $1 \mu \mathrm{m}$

same reason as the non-evaporating cases, the higher relaxation time of large particles. A difference in the circulation deposition trend is seen between the single and multi-group particles. For single group evaporating particles, the circulation reaches a maximum point at some early time and then gradually decreases. However, for the multi-group evaporating case, the circulation is seen to increase to a much later time. This shows that the evaporation effects are longer lived than the momentum equilibriation effects.

The enstrophy, $\Omega(\omega)$, is plotted for all the cases in Figure 3.8. It can be seen that for the single group, the rate of decrease in enstrophy is smaller in the evaporating case than the non-evaporating case. For the multi-group case, the enstrophy for the evaporating particles decreases at early time and then gradually starts to increase and becomes greater than the non-evaporating case. The decrease in enstrophy at early time is due to the rapid evaporation of the smallest size particle group, group 1, 


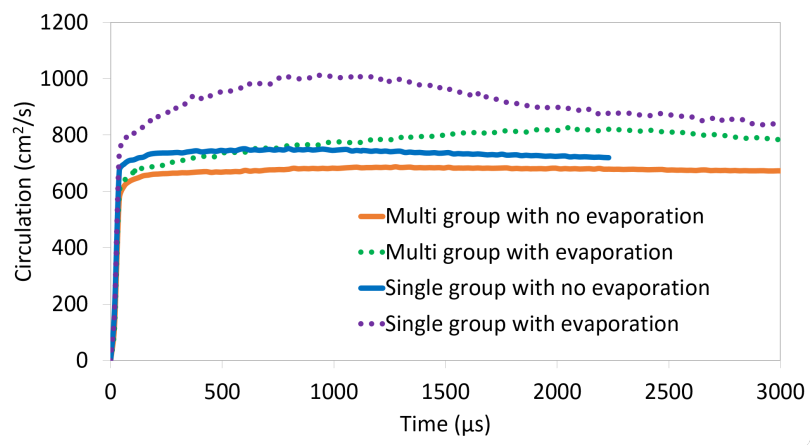

Figure 3.7: Circulation plot of the single and multiple group of particles with and without evaporation. The solid lines shows the plot without evaporation and the dotted lines are for the plots with evaporation

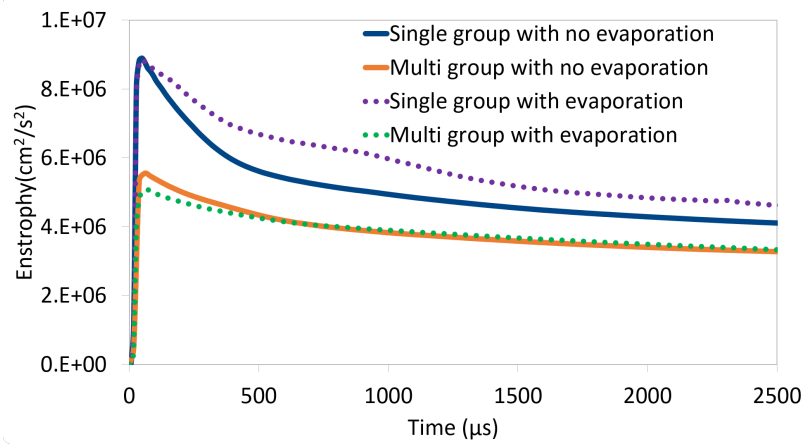

Figure 3.8: Enstrophy plot of the single and multiple group of particles with and without evaporation. The solid lines shows the plot without evaporation and the dotted lines are for the plots with evaporation

which shows that particle size modifies the evaporation effects. Overall the effect of evaporation on enstrophy is stronger for the single group case. This is likely because the single particle group exerts evaporation effects over a shorter period of time while the multi-group case exerts these effects over a longer time and larger area which would decrease the enstrophy source term and the effect of evaporation on it.

\subsubsection{Particle property histories}

The particle average diameter and temperature history for each particle group was studied for the evaporation cases. From Figure 3.9 it can be seen that the smallest diameter particles, group 1 , completely evaporate by about $t=200 \mu s$, group 2 
particles by about $t=1000 \mu \mathrm{s}$ and group 3 particles by about $t=2000 \mu \mathrm{s}$. Group 4 and group 5 particles do not evaporate completely even up to $t=4000 \mu s$. The diameter of all the droplets decreases smoothly up to a certain range and deviates. The temperature oscillation is due to the decrease in diameter of the particles to very small values. This causes the thermal mass of the particle to be small causing the temperature change of the droplet to be very high as a result of the convective and the evaporative energy transfer. The high temperature again causes the high evaporation rate which results in very high energy being removed from the droplet by the latent heat of vaporization undershooting the temperature of the droplet. This proceeds like a chain reaction which can ultimately break the code. The particle radius being in the non-continuum region aggravates the situation.

A smaller time step could ameliorate this issue but the required time step would become prohibitively small. Instead, to prevent this problem, a criteria was set for removal of the particles from the grid which completely evaporates them once they reach a critical low temperature. This method, although non-physical, has no major effect on the development of the instability. This is because the particles which are flagged for removal are too small, on the order of $0.01 \mu m$, to have any effect on the gas phase and would evaporate within a few time steps naturally. As all the particles of group 4 and 5 do not evaporate completely, the temperature oscillations for these groups is small.

The single particle case average properties show a different trend for the diameter change in comparison to the $0.9 \mu m$ particle group despite having nearly the same diameter. This shows that when there are multiple particle sizes each size groups effects the evaporation properties and rate of evaporation for the other groups. This is because particle groups fully evaporate at different times creating a scenario where contributions to the overall evaporation rate from each group suddenly end. Having multiple size groups also extends the period of time over which evaporation can in- 


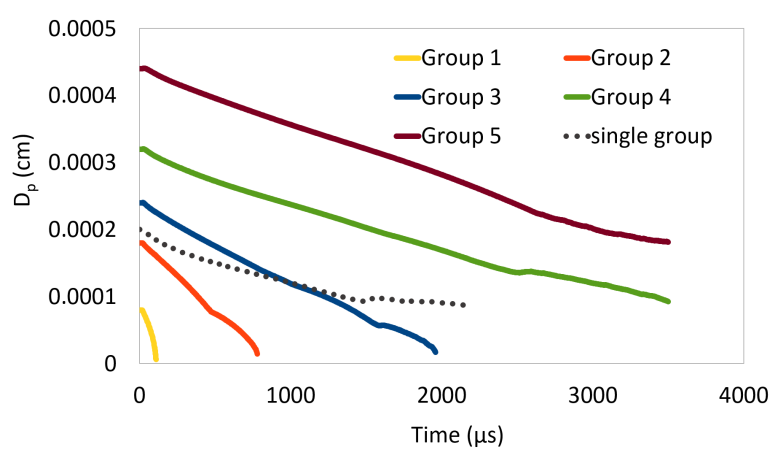

Figure 3.9: Time history of the droplet diameter

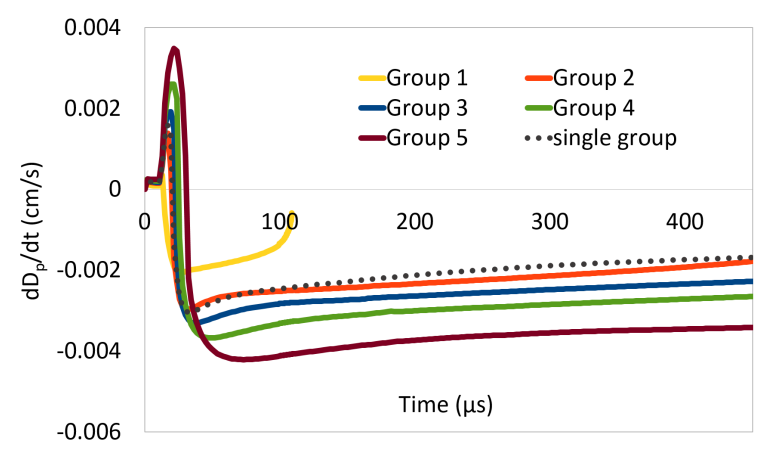

Figure 3.10: Time history of the rate of change of droplet diameter

fluence the instability and creates a slower overall evaporation rate for the instability.

In Figure 3.10, a small increase in diameter is seen before the shock wave strikes the particles due to a very small difference in the concentration of the vapor species in the gas field to the saturation concentration of water vapor at the gas temperature. This small condensation is negligible as no diameter increase is seen in Figure 3.9. The sudden drop in the rate of diameter increase is seen due to the shock wave reaching the particle position. The sudden increase in temperature of the particle is also seen at that instant from Figures 3.11 and 3.12. The rate of increase in temperature is fastest for the smallest size particle group. The temperature of the group 2 particles nearly aligns with the single group temperatures showing that unlike mass transfer, the temperature of the group is not dependent on the presence of multiple particle size groups. This is because temperature is driven mostly by convective heat transfer where as particle evaporation was driven by diffusion and species gradients. The 


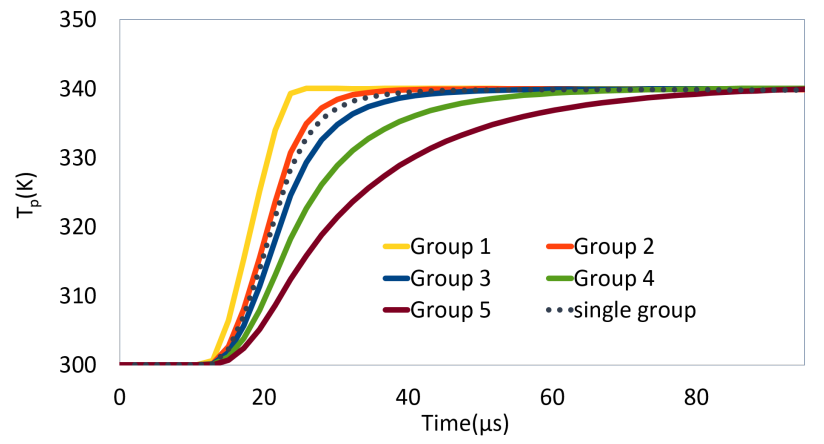

Figure 3.11: Time history of the droplet temperature

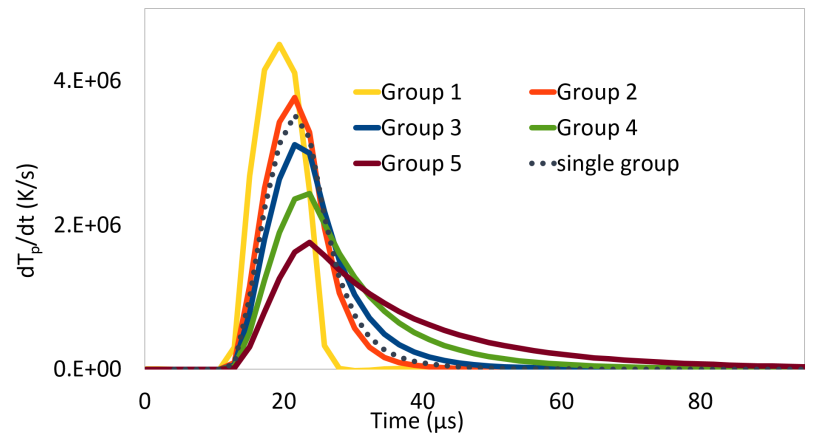

Figure 3.12: Time history of the gradient of droplet temperature

difference in the evaporation rate for group 2 and the single group then has little effect on their temperature history. The temperature of all the particles reaches an equilibrium temperature of about $340 K$ which is in psychrometric equilibrium with the post shock gas conditions.

\subsubsection{Late time qualitative comparison}

Finally, a late time qualitative comparison of the four different cases is presented to highlight the differences in properties among the cases. These differences would be important in subsequent accelerations of the interface which are common in many applications and can occur by a second shock or expansion wave driven acceleration. Four different gas variables, vapor species mass fraction, density, temperature, and vorticity are shown in Figure 3.13. The non-evaporating case does not have any 
significant changes in the density or temperature of the gas but the multi-group case does show a more diffuse interface created by the higher relaxation times of the larger particle groups. The multi-group case shows a slightly diminished vorticity compared to the single non-evaporating case. Evaporation, however, causes significant differences in the gas density, temperature, and vorticity.

The energy removed in the form of the latent heat during the evaporating cases causes a significant decrease in temperature and therefore an increase in density. The presence of the vapor species also modifies the gas density but due to our choice of species, water, this effect is small. The distribution of the density field is effected by the particle size distribution where the larger particles momentum lag and slower evaporation rate pulls the cold dense region of the interface closer to the core. For the single particle group this dense region is positioned towards the outside edge. The effective density difference between these cold regions and the rest of the seeded gas is only about $7 \%$ but this difference would more than double the Atwood number in these dense regions altering the growth of the instability if it were to be subjected to subsequent accelerations. All cases exhibit multiple vortex layers which lag behind the flow as seen by McFarland et al. [45]. These layers are more pronounced for the evaporating cases where it remains up stream of the primary interface for the single group case and is pulled downstream for the multi-group case. 


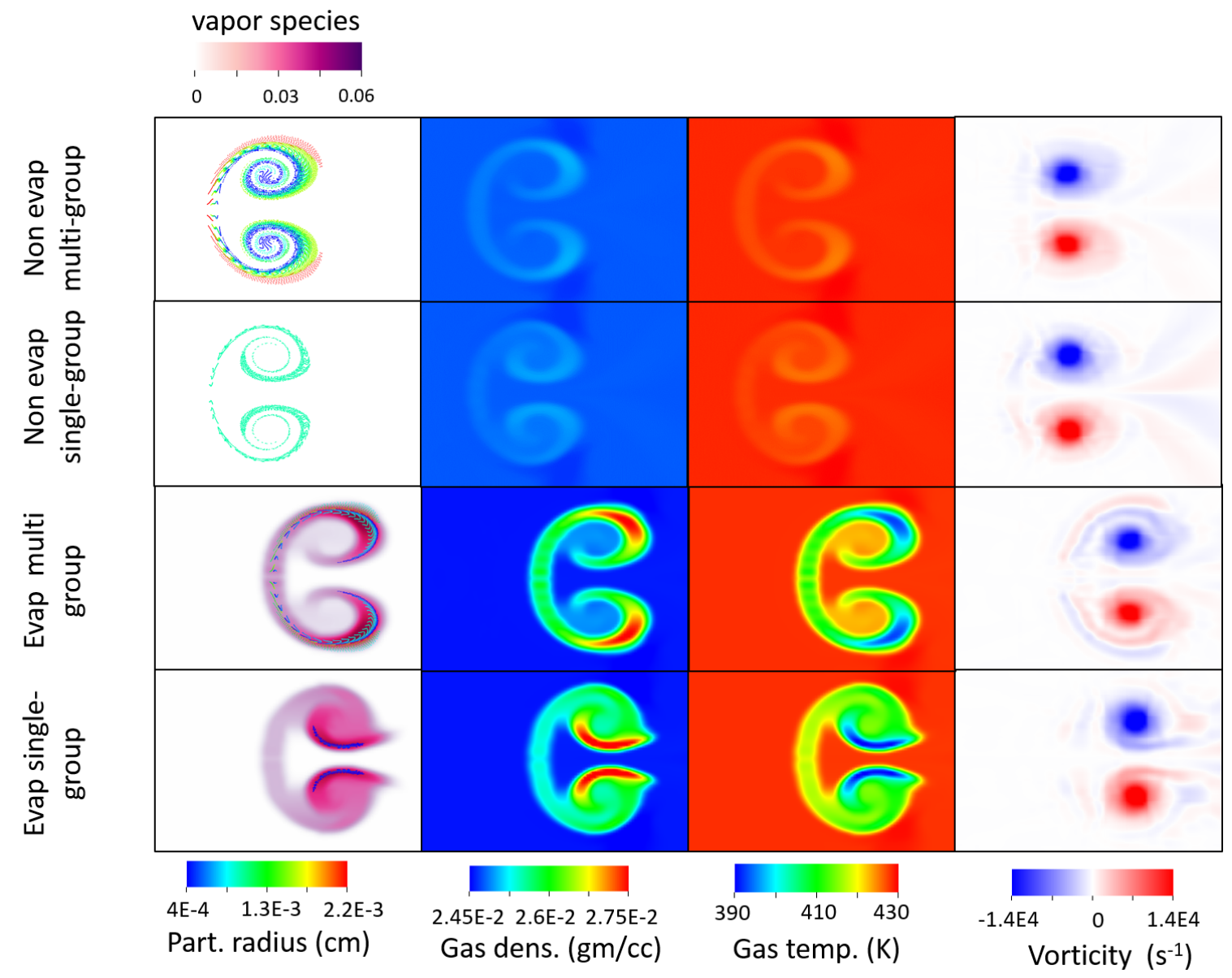

Figure 3.13: Pseudocolor plots at $\tau=2230 \mu \mathrm{s}$. Top: Non-evaporating multiple group particles. Top Center: Non-evaporating single group particles. Bottom center: Evaporating multiple group of particles. Bottom: Evaporating single group of particles. Left: Particle radius on top of the vapor species. Left center: Gas density field. Right center: Temperature of the gas. Right: Vorticity field

In the next chapter, this work will be extended to a three dimensional study of a spherical interface to replicate shock particle interactions occuring in natural phenomenon like the shock interaction with dust in supernovae remnants or Asymptotic Giant Branch stars. 


\section{Chapter 4}

\section{Multiphase hydrodynamic instability in 3D}

In this chapter a preliminary study of 3D shock particle interactions is carried out. An evolution of the spherical interface of water particles with random radii, which follow a lognormal distribution at completely random locations within the sphere, is studied. The data will further be analyzed in the future exploring different parameters to get new insights on shock particle interactions occuring in the nature. The spherical interface is chosen for study as it has various applications. It can be used to represent the dust in a gas cloud in interstellar medium, in the supernovae explosions, and in circumstellar shells of AGB stars.

\subsection{Background}

The shock bubble interaction (SBI) is one of the most widely studied RMI cases because of its wealth of applications from astrophysical phenomenon, to atmospheric physics, to inertial confinement fusion. Despite various natural phenamenon having the interaction of shock wave with dust clouds, there haven't been many studies on 
this case. McFarland et al. [45] and the study done in chapter 3 provides evidence that particles effect the morphology of the shock wave and the development of the hydrodynamic instability. The differences in the evolution of a gas and the particles can be highlighted by comparing the evolution of the particle interface with the equivalent dusty gas case. This encourages us to do a literature survey on the SBI.

Ranjan et al. [62] has given a review of the physics behind the shock bubble interaction. In addition, this section has also gives a brief review of various simulation and experimental studies of this case. The shock interaction leads to the compression of the bubble and the sudden jump in the thermodynamic properties like density, temperature, and pressure. The bubble also alters the propagation pattern of the wave by a nonlinear-acoustic mechanism which is related to the interface curvature and the acoustic impedence, $\mathbf{R}$, mismatch at the interface. Due to the acoustic impedence mismatch at the interface, the bubble acts as a diverging or converging gas lens. Acoustic impedence is a thermodynamic property and is defined as;

$$
\mathbf{R}=\rho_{\mathbf{g}} \mathbf{c}
$$

In equation $4.1, \mathbf{R}$ is the acoustic impedence, $\rho_{g}$ is the density of the gas and $c$ is the speed of sound.

On the diverging interface, a reflected wave and transmitted wave are formed. The reflected rarefraction waves generated at the downstream diverging interface of the bubble causes shock focusing at that end resulting in the development of an annulus. Klein et al. [63] did a computational study of the shock wave of Mach 10 propogating through the intercloud medium using the second-order-accurate Gondunov method with AMR. The results showed the crushing of the cloud due to vorticity generation by combined action of RTI and KHI. Niederhaus et al. [64] did a comprehensive study of the three dimensional shock bubble interaction at various Mach numbers and Atwood 
numbers. The results showed that the three dimensional features become significant at late times and these cannot be obtained from 2D simulations. The author also showed the significance of the shock refraction, reflection, and diffraction on the formation of primary and secondary vortex structures, which alter the evolution, preventing coherent structures to become chaotic.

Various experimental works have been done to verify the computational results and give new insight on the growth of bubble interface as it evolves towards turbulence. Ranjan et al. [65] through an experimental study showed the formation of a vortex ring, and a annulus at the downstream edge of a bubble containing Helium driven by a shock wave of $M 2.95$. The results also showed the presence of the secondary and tertiary vortex rings created by secondary baroclinic vorticity terms associated with the high Mach number shock accelerations. Haas and Sturtevant [66] did an experimental study of the shock interaction of an inhomogenous gas sphere and cylinder. Fluid inhomogenities modify the shock wave by modifying the reflection, refraction, and the diffraction pattern due to acoustic impedence mismatch at each infinitesimal inhomogenitiy, which are important for gas hydrodynamics. The author found that the axial jet annulus is more prominent in the sphere case than the cylinder gas. He also observed the vortex pairs in the sphere case to be more diffuse than the cylinder case. Layes et al. [67] quantified the mixing length, height of the bubble, vortex diameter, and spacing through an experimental study for negative, zero and positive density jump across the interface.

The discrete nature of the particles affects the shock waves in different ways than gas discontinuities. Shock reflection, refraction, and diffraction occur at each discrete particle. Boiko et al. [68] studied a shock interaction with a cloud of particles. The author concluded that the transmitted waves slow down because some amount of energy is spent in accelerating the particles. In addition, a negative pressure gradient is created inside the particle field that slows the reflected waves; which are composed 
of the compression and the rarefraction waves that are formed upstream of the particle cloud. This phenomenon is different than the gas bubble as the transmitted waves are independent of the acoustic impedence or the shape of the interface formed by a gas cloud [62]. Furthermore, the rarefraction waves in a gas bubble are formed only at the diverging interface whereas in the particles, they are formed by all the particles inside the cloud.

\subsection{Simulation setup}

It has been well researched that the particle sizes in nature roughly follow a lognormal distribution. In the previous chapter bins of particle radii called groups were used with the representative radius being the median radius of the particles in the group. Such arrangement allowed us to give a parcel size for each of the groups on the basis of the lognormal distribution. So, a distribution of the particle sizes could be achieved by a much smaller number of particles. In this study, 1.017E6 particles of random radii following a lognormal distribution with a median radius of $1 \mu \mathrm{m}$ are initialized. The particles are completely refined so as to have a 1:1 ratio of particles per parcel. Furthermore, the location of particles in the sphere are randomly assigned within radius $0.3 \mathrm{~cm}$. The average Atwood number of the particle gas mixture within the sphere at the initial conditions was found to be 0.08. The histogram of the particle radii at the initial condition is as shown in the Figure 4.1.

The simulation was run with the help of Argonne National Laboratory's supercomputer Vesta, on 32 nodes with 32 MPI processes per node. The simulation was run multithreaded with 4 threads per MPI processes. The setup command + ThreadBlockWithin="True" sets the multithreading environment in FLASH. In addition, the adaptive mesh refinement criteria in FLASH only has a grid refinement on the gradient of the variables. The grid refinement criteria was modified so as to obtain 


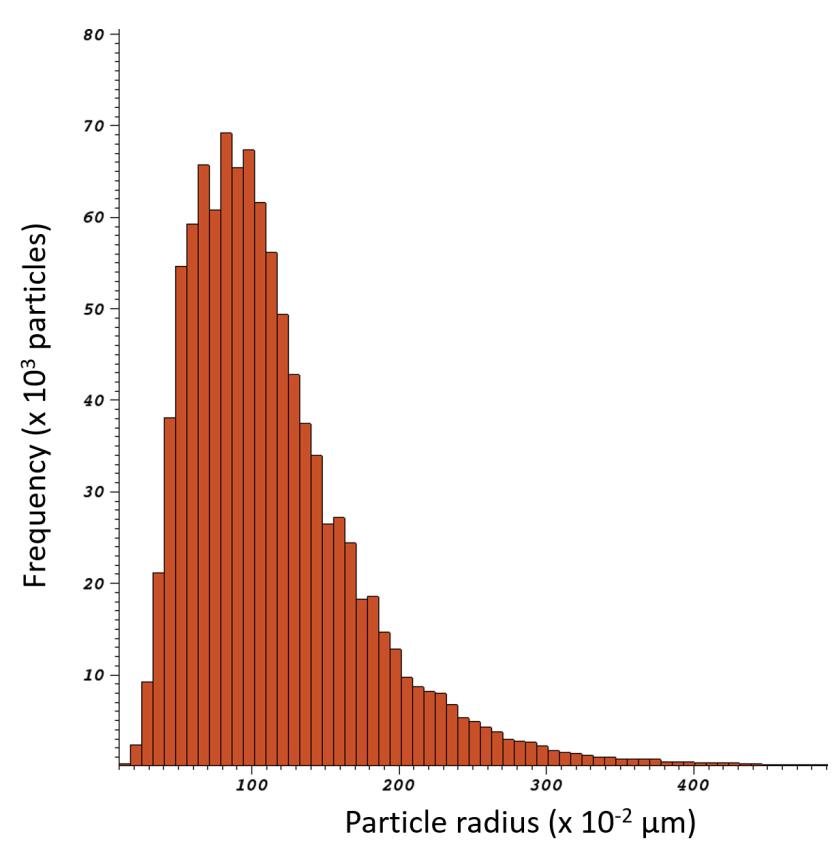

Figure 4.1: Histogram of the particles radii at initialization

the highest refinement only at the location where the particles are present. The MPI programming was done to share the information of the particle radius between the processors to find the minimum and maximum $X$ position of the particles, and the blocks within those distances were marked for refinement. The $Y$ and $Z$ direction were fully refined as artifical vorticity was seen at the interface of the refined and derefined blocks by eliminating large, slow, outliers. Since the median radius of our lognormal distribution is $1 \mu \mathrm{m}$, a cutoff radius of $5 \mu \mathrm{m}$ was chosen to save some computational time. The simulation domain is $100 \mathrm{~cm} \times 1 \mathrm{~cm} \times 1 \mathrm{~cm}$ and the simulation was run at 5 levels of refinement with 128 nodes per diameter. The number of zones changes during the simulation due to AMR with around 7 million zones at initialization.

The pressure, $P$, of the unshocked air is $101.325 \mathrm{KPa}$, and the temperature, $T_{g}$, is $300 \mathrm{~K}$. The sphere is shocked with a Mach 1.65 shock wave giving a post shock speed of $302 \mathrm{~m} / \mathrm{s}$. The average Atwood number, $A_{p g}$, during intialization is 0.08 . The problem is initialized with a tagged gas with the properties of air containing the 
particles at intial time. The initial setup of the 3D case is shown in Figure 4.2.

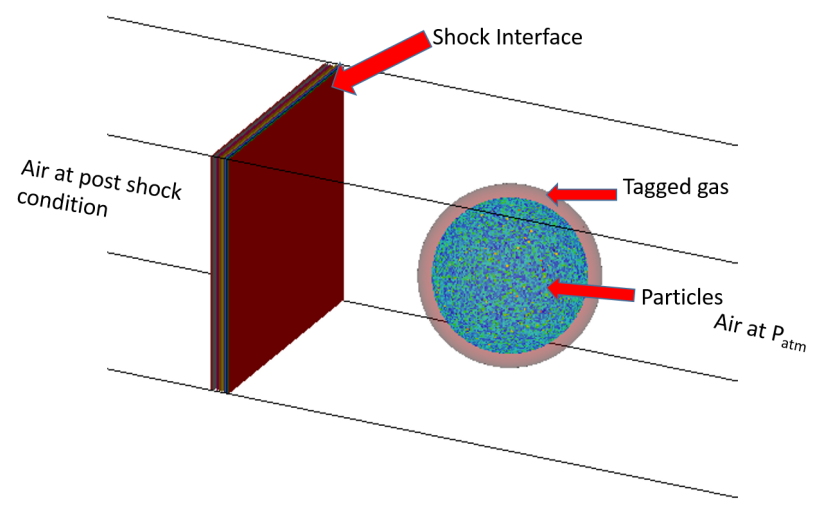

Figure 4.2: Intial setup of the 3D case showing the pressure contour and tagged gas contour containing the particles.

\subsection{Qualitative analysis}

The evolution of the interface is seen to be similar to the 2D shock cylinder problem. The rollups are formed due to the development of vorticity by the particle source term at the interface. The larger size particles lag behind the flow due to the higher velocity relaxation time. A direct comparision of the $3 \mathrm{D}$ case cannot be done with the $2 \mathrm{D}$ case as they are simulated at different effective Atwood numbers.

In Figure 4.3, the evolution of the particle interface is given. The top plot is the contour plot of the $5 \%$ water vapor fraction together with the particle radii. The contour of the vapor fraction is sliced in the $Z$ direction ( $X Y$ plane) for visualizing the internal formation of the particles and the vapor. The bottom plot is the contour plot of $5 \%$ tagged gas with the particle radii. The contour is reduced in opacity for clear visualization of the particles. From the figure a smooth gradient in the particle radii can be seen as the interface evolves. The smooth gradient is due to the larger number of particles initialized which allows them to smoothly follow the flow due to their lognormal distribution(4.1). Another interesting phenomenon that can be seen is that the particle interface width is larger at early times due to the particle lag, and 


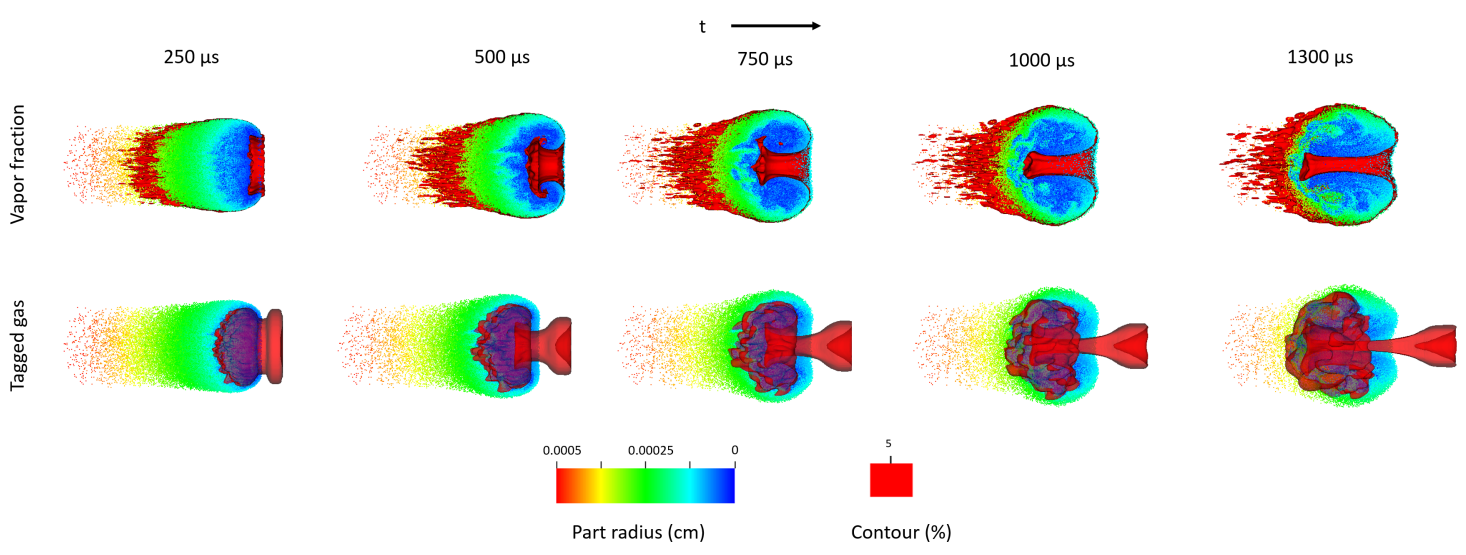

Figure 4.3: Contour plots of gas parameters with particles. Top: Counter plot of vapor fraction with slice at the center plane in $\mathrm{Z}$ direction for both the gas and particles. Bottom: Counter plot of the tagged gas with reduced opacity to visualize the particles in the center and the slice of the particles in the $\mathrm{Z}$ direction at the center plane.

decreases later in time. This is due to the secondary hydrodynamic instability growth between the particles of different sizes after the passage of the shock wave, which is a result of longer acceleration period of the particles due to drag. The growth can be seen as finger like structure which causes mixing and are more evident in the interface separating the small (blue) and large (green) particles.

The vapor contour has spikes at the upstream interface. The random location of the particles and the particle lag causes the formation of those spikes. The $5 \%$ vapor fraction contour at the location with the largest size particles cannot be seen due to their slow evaporation and low population. The tagged gas also forms the vortex ring with small scale structures at the surface due to the random size and location of the particles. The annulus is seen at the donwstream edge with a secondary vortex formed due to shock concentration by the rarefraction waves. However, the particles are not seen to follow the annulus. The rarefraction waves are generated at every particle location causing a weaker shock concentration at the downstream interface. So, contrary to the gas case, the concentrated shock wave cannnot make the particle follow the annulus. 
The Figure 4.4 show the effect of particles on the gas properties at different times. The plots are sliced at the centerplane in the $Z$ direction ( $X Y$ plane) for visualization. In the figure, the formation of the wavy small scale structures emitting out of the interface can be seen. This is due to the random distribution of the location of the particles. The interface is asymmeteric in the $Y$ direction from the centerline. There is randomness in the distribution of the density, temperature, and vapor phase in the interface. The alternating votex sheets are seen at ealy times which get pushed together at late time forming patches of high and low vorticity regions. This phenomenon can cause a decrease in overall vorticity of the interface due to competition between the negative and positive vorticity.

The vortex ring developed is more diffuse and a clear vortex ring cannot be seen at late time contrary to the cylinder case. A similar diffuse vortex ring for the sphere case was seen by Haas and Sturtevant [66] in their experiments. The small scale structures developed and the asymmetry in the gas properties is not due to the randomness of the particle locations and radii alone. The modification of the shock wave due to reflection, rarefraction, and the development of the compression waves also introduces the wavy structures. Furthemore, these small features appear in nature and can be seen more evident in the 3D simulations during the computational analysis. Three dimensional effects are significant as the effect of the axisymmetric components of the vorticity and the vortex stretching can only be observed numerically in 3D simulations. These components have a very high influence on the overall growth of the interface.

The cylindrical coordinate system provides better representation of the circulation of the vortex ring. The data in the cartesian coordinate system was converted to the cylindrical coordinate system by coordinate transformation. The $Z, Y$ and $X$ coordinates are transformed to $r, \theta$ and $z$. In the later discussions, only the vorticity and circulation are given in cylindrical coordinate system and the dimensions are 


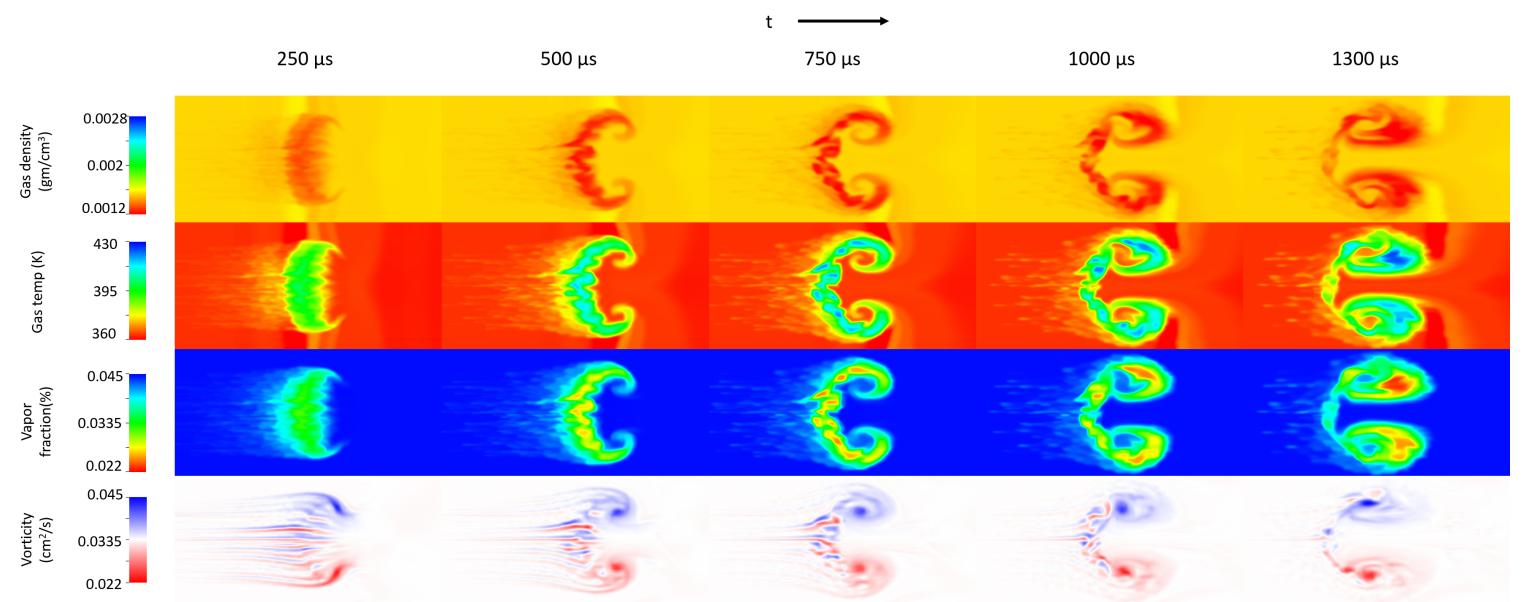

Figure 4.4: Pseudocolor plots of gas properties at various time. The plot is sliced at the centerplane in the $Z$ direction ( $X Y$ plane).

given in cartesian coordinate system. Figure 4.5 shows the vorticity in the $\theta$ direction at the centerplane in the cartesian $Z$ direction sliced by $X Y$ plane. The plot shows a strong vortex region at the rollups. The strong vortex regions vary from $54 \mathrm{~cm}$ to $54.6 \mathrm{~cm}$ which marks the region of higher particle concentration. Figure 4.6 gives the vorticity in the $\theta$ direction of the interface at late time, $\mathrm{t}=1.3 \mathrm{~ms}$, sliced by cartesian $Y Z$ planes at different $X$ locations. The $Y Z$ vortex slices are taken from $X$ distance of $54.0 \mathrm{~cm}$ with an increment of $0.1 \mathrm{~cm}$. At the base of the vortex, an alternating vortex patch is observed. A similar vortex distribution is seen in the next slice but has a slightly larger circumference. The strongest positive vorticity is observed at the midway region of $54.2 \mathrm{~cm}$ and $54.3 \mathrm{~cm}$. The effect of negative vorticity is minimal here. The positive vorticity dominates in the theta direction which dictates the shape of the interface and hence the vortex ring. The vorticity is strongest at a certain radius, $r$, as seen in the Figure 4.6. In the $r$ direction, most of the particles are concentrated at the vortex ring forming a high vortex region. 


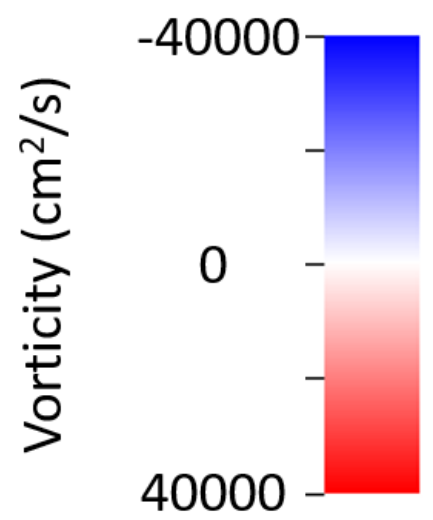

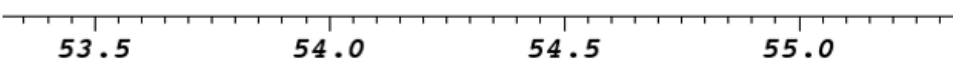

Figure 4.5: Vorticity plot at $\mathrm{t}=1.3 \mathrm{~ms}$. The domain is sliced at the centerplane in $Z$ direction ( $X Y$ plane).

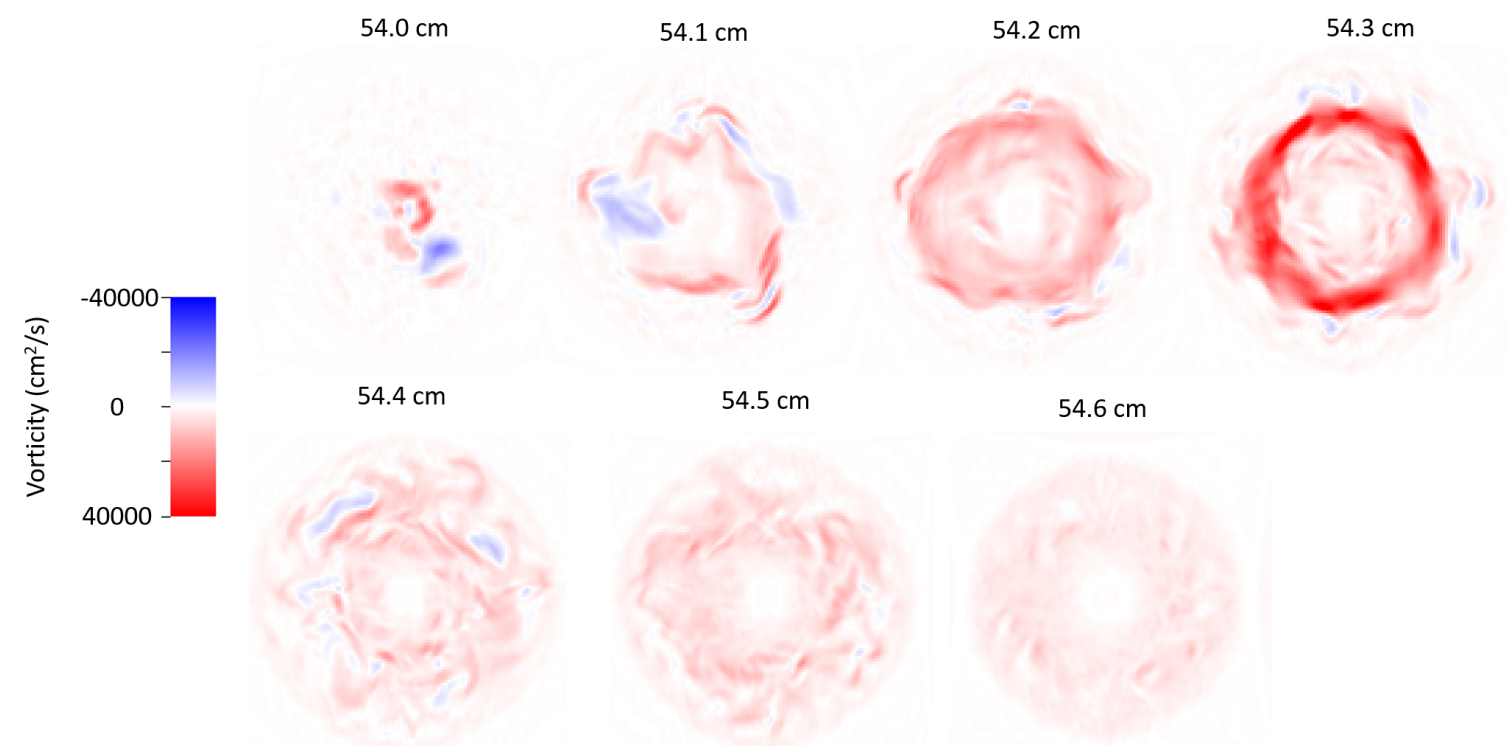

Figure 4.6: Vorticity plot at $\mathrm{t}=1.3 \mathrm{~ms}$. The domain is sliced at different locations in the $\mathrm{X}$ direction. 


\subsection{Quantitative analysis}

Figure 4.7 shows the circulation plot in the $\theta$ direction for the $3 \mathrm{D}$ shock particle sphere interaction. The circulation plot shows a similar trend as previously seen in the shock cylinder interaction in $2 \mathrm{D}$. There is an initial spike at early time due to the interaction of the shock wave. As time progresses, the circulation decays. However, For the $z$ direction, there is a continous increase in circulation with time. An increase in circulation is seen as an integral of only the positive component of vorticity is done. However, net vorticity in the $z$ direction is close to zero.

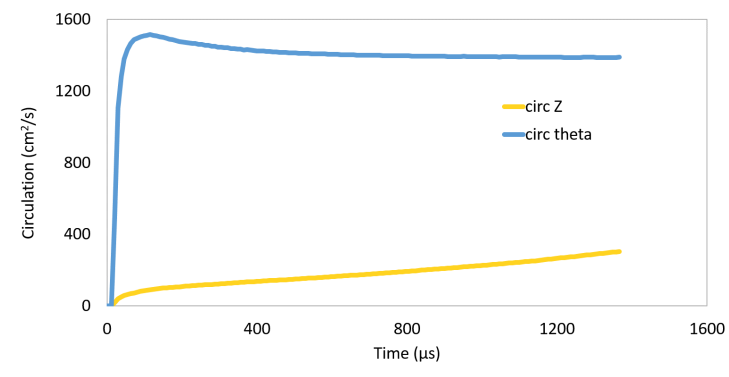

Figure 4.7: Circulation plots in $\theta$ and $x$ direcions.

Various analytical models are available to calculate the circulation for the SBI. Picone et al. [69, 70] gave a model for calculating the circulation by integrating the baroclinic source term. Yang et al. [71] also gave a model for calculating the circulation using a similar method. Samtaney and Zabusky [72] take a different approach in calculating the circulation. This model, with the help of scaling arguments based on shock polar analysis and numerical simulation, captures the shock refraction effect. This analysis can be used to calculate the circulation deposition.

The interface width plot in Figure 4.8 shows the small decrease in the interface at very early time. This is due to the squeezing of the particle region as shock wave strikes them. After that there is a constant increase in the interface width due to the differences in the velocity relaxation times. The growth of the secondary 
hydrodynamic instability between different particle sizes starts to pull the particles together constantly decreasing the interface width at later times.

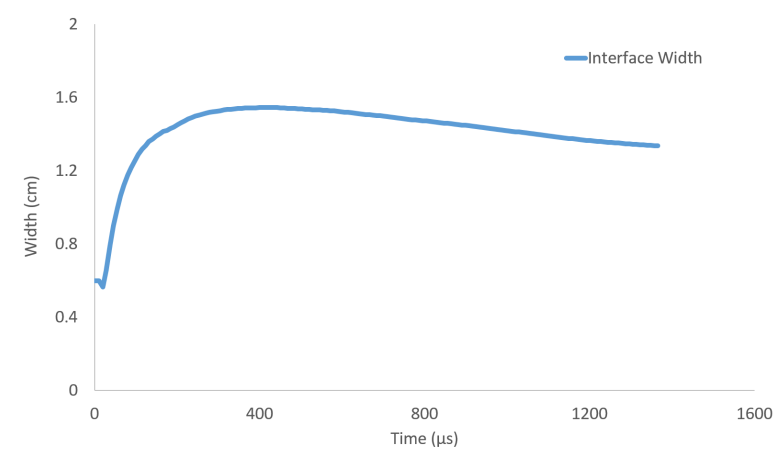

Figure 4.8: The width of the particle interface with time.

The work done in chapter 4 is still in progress. In the future, a gas equivalent of our particle model will be used to compare the circulation obtained in our simulation with the analytical model. The circulation obtained from dusty gas simulation will be compared with the particle case and the analytical model to exactly determine the particle effects in the evolution of the interface in three dimensions. In addition, the statistical analysis of the data will be done to characterize the turbulence during the evolution of SDMI in 3D. 


\section{Chapter 5}

\section{Summary and Conclusions}

A model for the interaction of evaporating particles with a surrounding carrier gas in a shock driven environment has been developed and used to study the effects of evaporation and particle size distribution on a simple shock driven circular and spherical interface. The Particle-in-Cell method was combined with the piecewise parabolic method for simulation of shock driven multiphase instabilities to obtain a stable solution of Lagrangian particles in a gas modeled on an Eulerian grid. The method given by Andrews and O'rourke [20] was modified for the shock-particle interaction resulting in a simpler closed coupled momentum equation. The Kliatchko model for particle drag with the modification for the shock wave given by Carter et al. [54] was used in our model. The Ranz-Marshall correlation was used to predict convective heat transfer to the particles and the Antoine equation and ClausiusClapeyron equations were used to predict the energy absorbed in evaporation of the particle mass. These equations were coupled with the fluid equations discretized on an Eulerian grid.

Our particle models were implemented in the open source hydrodynamics code FLASH, developed by University of Chicago's FLASH center. Validation was performed for a single particle case which compared the temperature and velocity history 
obtained from simulations to an analytical solution, and showed good agreement. Furthermore, a psychrometric analysis of the evaporation model was done by simulating the evaporation of water droplets in dry air at $300 \mathrm{~K}$ and showed good agreement with the established psychrometric properties of humid air. The models were then validated with a qualitative comparison to the shock-driven cylindrical multiphase perturbation experiments of Vorobieff et al. [1]. The results showed that particle size distribution plays an important role in the development of hydrodynamic instability. The multi-group particle simulation showed about an $11 \%$ decrease in circulation and showed a mixing region more similar to that which was found in the previous experiments.

The decrease in circulation due to the presence of the larger size particles in the multi-group case was explored further in simple single particle interface simulations. From an enstrophy analysis of $1 \mu m$ and $5 \mu m$ particle interfaces, it was seen that the $1 \mu m$ particles produced a higher enstrophy. This higher enstrophy was attributed to the alignment of the vorticity field and vorticity source term created by the particlegas momentum equilibriation. For large particles the vorticity source term advects through the flow relative to the gas and is not well aligned until late times resulting in a decreased enstrophy source term. The small particles were also found to exhibit a much higher degree of enstrophy dissipation at early times. The dissipation term was examined further to provide an estimate of the effective numerical viscosity. This analysis suggests that the large particles create a higher effective viscosity than the small particles possibly due to the higher relaxation times. At later times, finger like structures are seen to grow and randomly mix. The random gas mixing will be characterized by the evolution of the turbulence, but the random particle mixing will alter the gas properties which develops random asymmetric growth leading towards the development of the turbulence.

The effect of evaporation was examined for a $2 \mathrm{D}$ circular interface for the single 
particle size and multiple particle size group cases. Evaporation was found to create an increase in circulation compared to the corresponding non-evaporating cases. Evaporation also cooled the gas interface and created a gas density gradient which could lead to a strengthened instability in the case of additional interface accelerations by additional shock or expansion wave interactions as is common in many applications. This increase in the interface gas density gradient leads to additional baroclinic vorticity deposition and an increase in the strength of the vorticity source term. The rate of evaporation of different sized groups was found to extend the phase equilibriation period for the multi-group case with larger particles. This resulted in a different morphology of the interface at late times.

The 3D simulations were performed to study the shock driven multiphase hydrodynamic instability with the parameters set to represent the occurance of the particles in nature i.e. an interface with random radii following a lognormal distribution at random locations. The simulation was run at a parcel to particle size of 1:1. The spherical interface was found to evolve differently than the cylinderical interface as it was more diffuse. An asymmetric gas property field was observed which can be caused by the random particle distribution or the compression and rarefraction waves at the interface. The secondary hydrodynamic instability was also found to play an effect in the evolution of the interface. The interface width decreases in size at later times due to the effect of secondary hydrodynamic instability. The circulation plot shows a similar trend to the 2D cylinder case. There is a spike in circulation produced at early time which decays as time progresses. The vorticity in the $z$ direction was also found to increase with time which is interesting as we expect the gas field to not produced any vorticity in that direction.

This study provides new insights on the particle effect during SDMI. The multiphase effects were found play an important role in the evolution of the particle interface. The larger particles cause slower evolution of interface than the smaller 
size particles as they alter the rate of deposition of the vorticity. So, particle physics and their effects cannot be ignored while using them as a gas tracer. The extend of the particle effects can be understood from the reaction time of the particles to the flow. Larger particles which are slow to react have greater effect on the flow than the smaller particles. Evaporation of the particle and 3D effects were also found to considerably affect the evolution. This is a work in progress and further study of the particle effects in 3D will be done in the future. In addition, clustering of the particles during the evolution of the interface towards turbulence will also be studied. This will be done by doing statistical analysis for particle turbulence characterization.

In the future, futher development of the program will be done by supplementing it with new models for particle condensation, radiation, collision, and breakup. In addition, through computational study, the effect of shock waves in the processing of the dust in supernovae explosions and Asymptotic Giant Branch stars will be studied. Furthermore, the effect of the complex accelerations by the blast wave and the re-shock on the particle interface will also be studied.

\subsection{Acknowledgments}

The author would like to thank the Missouri Research Board for their grant supporting this work. The software used in this work was developed in part by the DOE NNSA ASC- and DOE Office of Science ASCR-supported Flash Center for Computational Science at the University of Chicago. The visualization program used for this work is VisIt developed by Lawerence Livermore National Laboratory. The Author would also like to thank Prof. Vorobieff for his consultation and discussion of his experimental work, and W J. Black and N. Denissen for their consultation and discussion of particle simulation models. The author would also like to thank University of Missouri Bioinformatics Consortium for graning access to Lewis3 and directors 
of discretionary allocation at Argonne National Laboratory for granting access to Argonne Leadership Computing Facilities. 


\section{Bibliography}

[1] Peter Vorobieff, Michael Anderson, Joseph Conroy, Ross White, C Randall Truman, and Sanjay Kumar. Vortex formation in a shock-accelerated gas induced by particle seeding. Physical review letters, 106(18):184503, 2011.

[2] Rayleigh Lord et al. The theory of sound, 1945.

[3] Geoffrey I Taylor. The viscosity of a fluid containing small drops of another fluid. Proceedings of the Royal Society of London. Series A, Containing Papers of a Mathematical and Physical Character, 138(834):41-48, 1932.

[4] $\mathrm{H}$ von Helmholtz. Lxiii. on integrals of the hydrodynamical equations, which express vortex-motion. The London, Edinburgh, and Dublin Philosophical Magazine and Journal of Science, 33(226):485-512, 1867.

[5] Robert D Richtmyer. Taylor instability in shock acceleration of compressible fluids. Communications on Pure and Applied Mathematics, 13(2):297-319, 1960.

[6] EE Meshkov. Instability of the interface of two gases accelerated by a shock wave. Fluid Dynamics, 4(5):101-104, 1969.

[7] J Kane, RP Drake, and BA Remington. An evaluation of the richtmyermeshkov instability in supernova remnant formation. The Astrophysical Journal, 511(1):335, 1999. 
[8] Stéphane Zaleski and Philippe Julien. Numerical simulation of rayleigh-taylor instability for single and multiple salt diapirs. Tectonophysics, 206(1):55-69, 1992.

[9] VA Smalyuk, DT Casey, DS Clark, MJ Edwards, SW Haan, A Hamza, DE Hoover, WW Hsing, O Hurricane, JD Kilkenny, et al. First measurements of hydrodynamic instability growth in indirectly driven implosions at ignitionrelevant conditions on the national ignition facility. Physical review letters, 112(18):185003, 2014.

[10] Angela K Speck and Anne M Hofmeister. Processing of presolar grains around post-asymptotic giant branch stars: silicon carbide as the carrier of the 21 micron feature. The Astrophysical Journal, 600(2):986, 2004.

[11] Devin W Silvia, Britton D Smith, and J Michael Shull. Numerical simulations of supernova dust destruction. i. cloud-crushing and post-processed grain sputtering. The Astrophysical Journal, 715(2):1575, 2010.

[12] Marco Bocchio, Anthony P Jones, and Jonathan D Slavin. A re-evaluation of dust processing in supernova shock waves. Astronomy $\mathcal{E}$ Astrophysics, 570:A32, 2014.

[13] Yongtao Chen, Haibo Hu, Tiegang Tang, Guowu Ren, Qingzhong Li, Rongbo Wang, and William T Buttler. Experimental study of ejecta from shock melted lead. Journal of Applied Physics, 111(5):053509, 2012.

[14] MB Zellner, W Vogan McNeil, GT Gray III, DC Huerta, NSP King, GE Neal, SJ Valentine, JR Payton, J Rubin, GD Stevens, et al. Surface preparation methods to enhance dynamic surface property measurements of shocked metal surfaces. Journal of Applied Physics, 103(8):083521, 2008. 
[15] MB Zellner and WT Buttler. Exploring richtmyer-meshkov instability phenomena and ejecta cloud physics. Applied Physics Letters, 93(11):114102, 2008.

[16] Kenneth H Wohletz. Mechanisms of hydrovolcanic pyroclast formation: grainsize, scanning electron microscopy, and experimental studies. Journal of Volcanology and Geothermal Research, 17(1):31-63, 1983.

[17] Joseph Yang, Toshi Kubota, and Edward E Zukoski. Applications of shockinduced mixing to supersonic combustion. AIAA journal, 31(5):854-862, 1993.

[18] Andrzej Gardzilewicz, Michal Kolovratnik, et al. Numerical and experimental investigations of steam condensation in lp part of a large power turbine. Journal of Fluids Engineering, 131(4):041301, 2009.

[19] Michael Colarossi, Nathaniel Trask, David P Schmidt, and Mark J Bergander. Multidimensional modeling of condensing two-phase ejector flow. international journal of refrigeration, 35(2):290-299, 2012.

[20] MJ Andrews and PJ O'rourke. The multiphase particle-in-cell (mp-pic) method for dense particulate flows. International Journal of Multiphase Flow, 22(2):379$402,1996$.

[21] S. Balachandar and John K. Eaton. Turbulent Dispersed Multiphase Flow. Annual Review of Fluid Mechanics, 42(1):111-133, January 2010.

[22] Dimitri Gidaspow. Multiphase flow and fluidization: continuum and kinetic theory descriptions. Academic press, 1994.

[23] GK Batchelor. A new theory of the instability of a uniform fluidized bed. Journal of Fluid Mechanics, 193:75-110, 1988. 
[24] Martha W Evans, Francis H Harlow, and Eleazer Bromberg. The particle-incell method for hydrodynamic calculations. Technical report, DTIC Document, 1957.

[25] DM Snider. An incompressible three-dimensional multiphase particle-in-cell model for dense particle flows. Journal of Computational Physics, 170(2):523549, 2001.

[26] NA Patankar and DD Joseph. Modeling and numerical simulation of particulate flows by the eulerian-lagrangian approach. International Journal of Multiphase Flow, 27(10):1659-1684, 2001.

[27] Dale M Snider, Samuel M Clark, and Peter J O'Rourke. Eulerian-lagrangian method for three-dimensional thermal reacting flow with application to coal gasifiers. Chemical engineering science, 66(6):1285-1295, 2011.

[28] Anthony A Amsden, PJ O'rourke, and TD Butler. Kiva-ii: A computer program for chemically reactive flows with sprays. Technical report, Los Alamos National Lab., NM (USA), 1989.

[29] Anthony A Amsden. Kiva-3v: A block-structured kiva program for engines with vertical or canted valves. Technical report, Los Alamos National Lab., NM (United States), 1997.

[30] LD Cloutman. A numerical model of particulate transport. Technical report, Lawrence Livermore National Lab., CA (USA), 1991.

[31] Richard S Miller, K Harstad, and J Bellan. Evaluation of equilibrium and nonequilibrium evaporation models for many-droplet gas-liquid flow simulations. International Journal of Multiphase Flow, 24(6):1025-1055, 1998. 
[32] Joe J Monaghan. Smoothed particle hydrodynamics. Reports on progress in physics, 68(8):1703, 2005.

[33] Seiichi Koshizuka and Y Oka. Moving-particle semi-implicit method for fragmentation of incompressible fluid. Nuclear science and engineering, 123(3):421-434, 1996.

[34] M. Stanic, J. McFarland, R. F. Stellingwerf, J. T. Cassibry, D. Ranjan, R. Bonazza, J. A. Greenough, and S. I. Abarzhi. Non-uniform volumetric structures in Richtmyer-Meshkov flows. Physics of Fluids, 25(10):106107, 2013.

[35] BJ Balakumar, GC Orlicz, JR Ristorcelli, S Balasubramanian, KP Prestridge, and CD Tomkins. Turbulent mixing in a richtmyer-meshkov fluid layer after reshock: velocity and density statistics. Journal of Fluid Mechanics, 696:67-93, 2012.

[36] JW Jacobs, VV Krivets, V Tsiklashvili, and OA Likhachev. Experiments on the richtmyer-meshkov instability with an imposed, random initial perturbation. Shock Waves, 23(4):407-413, 2013.

[37] N Haehn, C Weber, J Oakley, M Anderson, D Ranjan, and R Bonazza. Experimental investigation of a twice-shocked spherical gas inhomogeneity with particle image velocimetry. Shock Waves, 21(3):225-231, 2011.

[38] Jacob McFarland, David Reilly, Skylar Creel, Christopher McDonald, Thomas Finn, and Devesh Ranjan. Experimental investigation of the inclined interface richtmyer-meshkov instability before and after reshock. Experiments in fluids, $55(1): 1-14,2014$.

[39] David Reilly, Jacob McFarland, Mohammad Mohaghar, and Devesh Ranjan. The effects of initial conditions and circulation deposition on the inclined-interface 
reshocked richtmyer-meshkov instability. Experiments in Fluids, 56(8):1-16, 2015.

[40] Jacob A McFarland, David Reilly, Wolfgang Black, Jeffrey A Greenough, and Devesh Ranjan. Modal interactions between a large-wavelength inclined interface and small-wavelength multimode perturbations in a richtmyer-meshkov instability. Physical Review E, 92(1):013023, 2015.

[41] J A McFarland, J A Greenough, and D Ranjan. Investigation of the initial perturbation amplitude for the inclined interface RichtmyerMeshkov instability. Physica Scripta, T155:014014, July 2013.

[42] M Anderson, Peter Vorobieff, CR Truman, C Corbin, G Kuehner, P Wayne, J Conroy, R White, and S Kumar. An experimental and numerical study of shock interaction with a gas column seeded with droplets. Shock Waves, 25(2):107-125, 2015.

[43] Satoshi Ukai, Kaushik Balakrishnan, and Suresh Menon. On richtmyer-meshkov instability in dilute gas-particle mixtures. Physics of Fluids (1994-present), 22(10):104103, 2010.

[44] JC Schulz, KC Gottiparthi, and S Menon. Richtmyer-meshkov instability in dilute gas-particle mixtures with re-shock. Physics of Fluids (1994-present), 25(11):114105, 2013.

[45] Jacob A McFarland, Wolfgang J Black, Jeevan Dahal, and Brandon E Morgan. Computational study of the shock driven instability of a multiphase particle-gas system. Physics of Fluids (1994-present), 28(2):024105, 2016.

[46] Hitoshi Miura, Taishi Nakamoto, and Hajime Susa. A shock-wave heating model for chondrule formation: effects of evaporation and gas flows on silicate particles. Icarus, 160(2):258-270, 2002. 
[47] Allard Jan Van Marle, Zakaria Meliani, Rony Keppens, and Leen Decin. Computing the dust distribution in the bow shock of a fast-moving, evolved star. The Astrophysical Journal Letters, 734(2):L26, 2011.

[48] Phillip Colella and Paul R Woodward. The piecewise parabolic method (ppm) for gas-dynamical simulations. Journal of computational physics, 54(1):174-201, 1984.

[49] B Fryxell, K Olson, P Ricker, FX Timmes, M Zingale, DQ Lamb, P MacNeice, R Rosner, JW Truran, and H Tufo. Flash: An adaptive mesh hydrodynamics code for modeling astrophysical thermonuclear flashes. The Astrophysical Journal Supplement Series, 131(1):273, 2000.

[50] M Parmar, A Haselbacher, and S Balachandar. Improved drag correlation for spheres and application to shock-tube experiments. Aiaa Journal, 48(6):1273$1276,2010$.

[51] G Jourdan, L Houas, O Igra, J-L Estivalezes, C Devals, and EE Meshkov. Drag coefficient of a sphere in a non-stationary flow: new results. In Proceedings of the Royal Society of London A: Mathematical, Physical and Engineering Sciences, volume 463, pages 3323-3345. The Royal Society, 2007.

[52] Nikolaj Albertovič Fuks. The mechanics of aerosols. Dover Publications, 1989.

[53] George Rudinger. Effective drag coefficient for gas-particle flow in shock tubes. Journal of Basic Engineering, 92(1):165-172, 1970.

[54] RT Carter, PS Jandir, and ME Kress. Estimating the drag coefficients of meteorites for all mach number regimes. In Lunar and Planetary Science Conference, volume 40, page 2059, 2009. 
[55] Peter John O'Rourke. Collective drop effects on vaporizing liquid sprays. Technical report, Los Alamos National Lab., NM (USA), 1981.

[56] WE Ranz and WR Marshall. Evaporation from drops. Chemical Engineering Progress, 48(3):141446, 1952.

[57] Clayton T Crowe, John D Schwarzkopf, Martin Sommerfeld, and Yutaka Tsuji. Multiphase flows with droplets and particles. CRC press, 2011.

[58] ER Gilliland. Diffusion coefficients in gaseous systems. Industrial \& Engineering Chemistry, 26(6):681-685, 1934.

[59] Daniel R Stull. Vapor pressure of pure substances. organic and inorganic compounds. Industrial \& Engineering Chemistry, 39(4):517-540, 1947.

[60] Chia-Tsun Liu and William T. Lindsay Jr. Vapor pressure of deuterated water from 106 to 300.deg. Journal of Chemical \& Engineering Data, 15(4):510-513, 1970.

[61] Lawrence D Cloutman. Analytical solutions for the trajectories and thermal histories of unforced particulates. American Journal of Physics, 56(7):643-645, 1988.

[62] Devesh Ranjan, Jason Oakley, and Riccardo Bonazza. Shock-bubble interactions. Annual Review of Fluid Mechanics, 43:117-140, 2011.

[63] Richard I Klein, Christopher F McKee, and Philip Colella. On the hydrodynamic interaction of shock waves with interstellar clouds. 1: Nonradiative shocks in small clouds. The Astrophysical Journal, 420:213-236, 1994.

[64] John HJ Niederhaus, JA Greenough, JG Oakley, D Ranjan, MH Anderson, and R Bonazza. A computational parameter study for the three-dimensional shockbubble interaction. Journal of Fluid Mechanics, 594:85-124, 2008. 
[65] Devesh Ranjan, John Niederhaus, Bradley Motl, Mark Anderson, Jason Oakley, and Riccardo Bonazza. Experimental investigation of primary and secondary features in high-mach-number shock-bubble interaction. Physical review letters, 98(2):024502, 2007.

[66] J-F Haas and Bradford Sturtevant. Interaction of weak shock waves with cylindrical and spherical gas inhomogeneities. Journal of Fluid Mechanics, 181:41-76, 1987.

[67] Guillaume Layes, Georges Jourdan, and Lazhar Houas. Experimental investigation of the shock wave interaction with a spherical gas inhomogeneity. Physics of Fluids (1994-present), 17(2):028103, 2005.

[68] VM Boiko, VP Kiselev, SP Kiselev, AN Papyrin, SV Poplavsky, and VM Fomin. Shock wave interaction with a cloud of particles. Shock Waves, 7(5):275-285, 1997.

[69] JM Picone and JP Boris. Vorticity generation by shock propagation through bubbles in a gas. Journal of Fluid Mechanics, 189:23-51, 1988.

[70] JM Picone, ES Oran, JP Boris, and TR Young Jr. Theory of vorticity generation by shock wave and flame interactions. Technical report, DTIC Document, 1984.

[71] Joseph Yang, Toshi Kubota, and Edward E Zukoski. A model for characterization of a vortex pair formed by shock passage over a light-gas inhomogeneity. Journal of Fluid Mechanics, 258:217-244, 1994.

[72] Ravi Samtaney and Norman J Zabusky. Circulation deposition on shockaccelerated planar and curved density-stratified interfaces: models and scaling laws. Journal of Fluid Mechanics, 269:45-78, 1994. 


\section{Appendix A}

\section{Particle Models and Subroutines}

\section{A.1 Momentum Exchange}

The momentum exchange model that we built in the FLASH code is located at

source/Particles/ParticlesMain/active/massive/hybrid. The contents of the directory are;

- energyexchange (directory containing energy exchange model files)

- Config

- Makefile

- pt_picInterface

- pt_picData.F90

- pt_picInit.F90

- pt_calculateVar.F90

- pt_calculateDrag.F90 
- pt_calculateVarTemp.F90

- pt_calculateTemp.F90

- pt_picApplyBoundary

- pt_picTools

- pt_picAdvanceVel.F90

- pt_advanceCharged.F90

The detail subroutines are included below.

\section{A.1.1 Config}

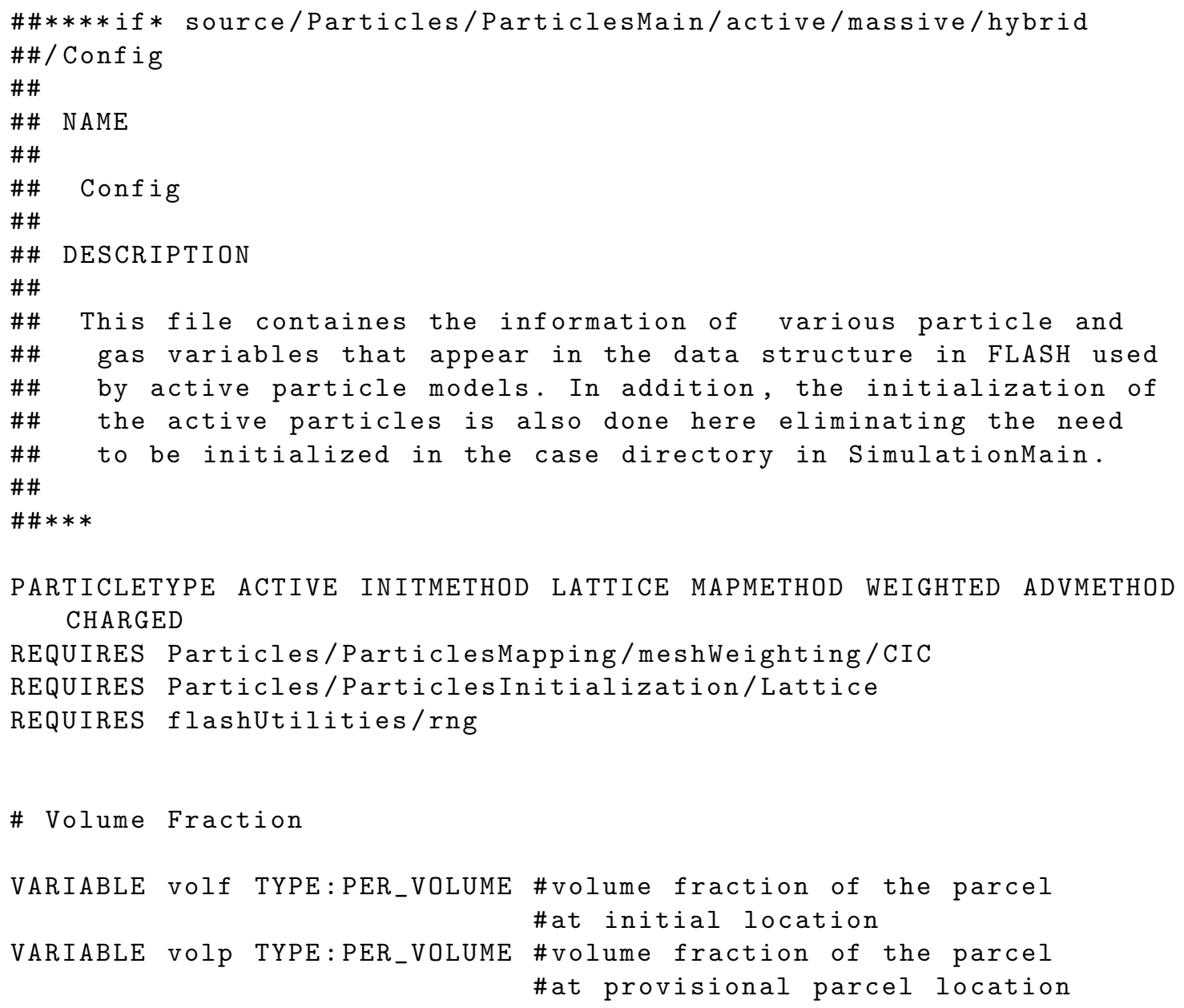




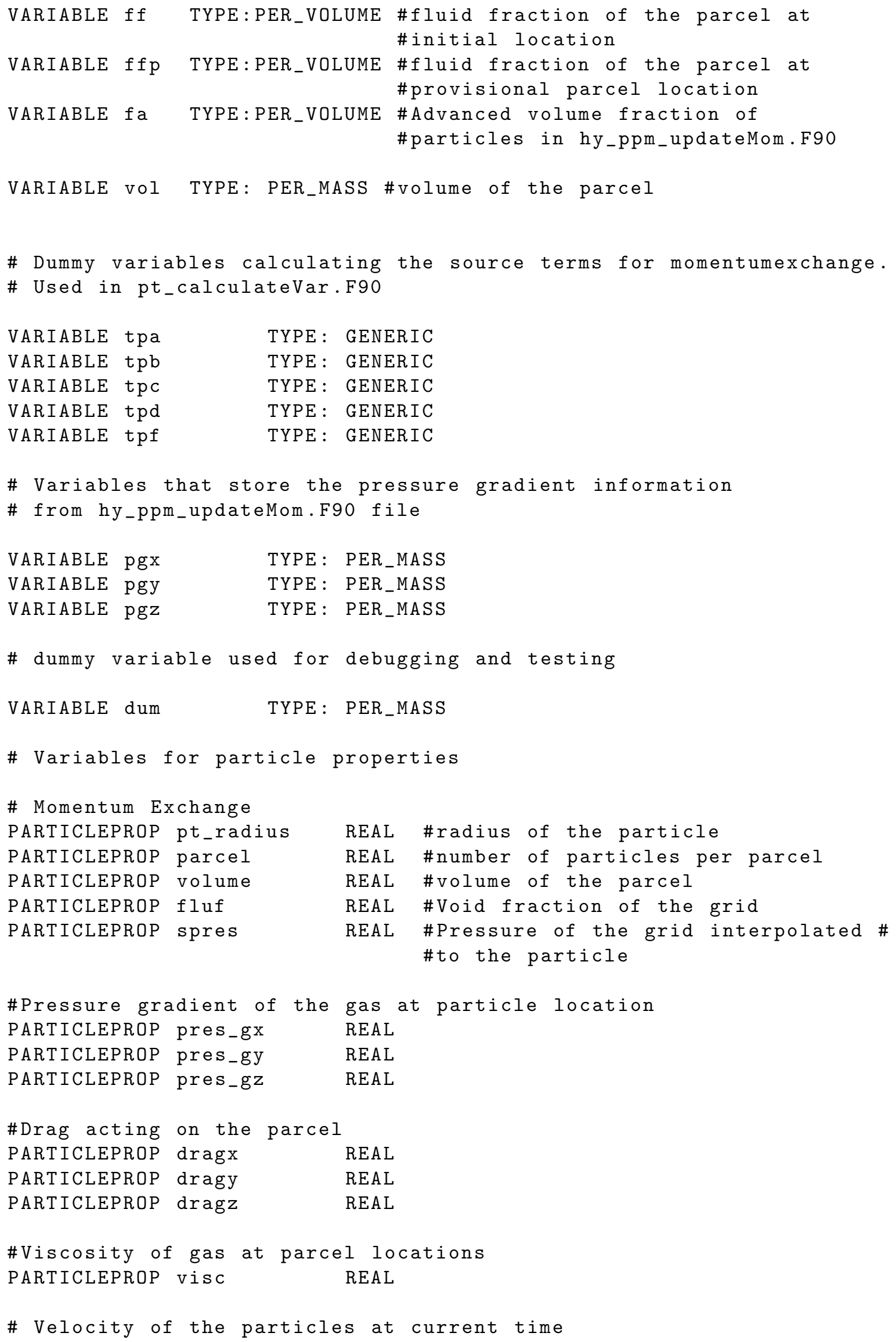




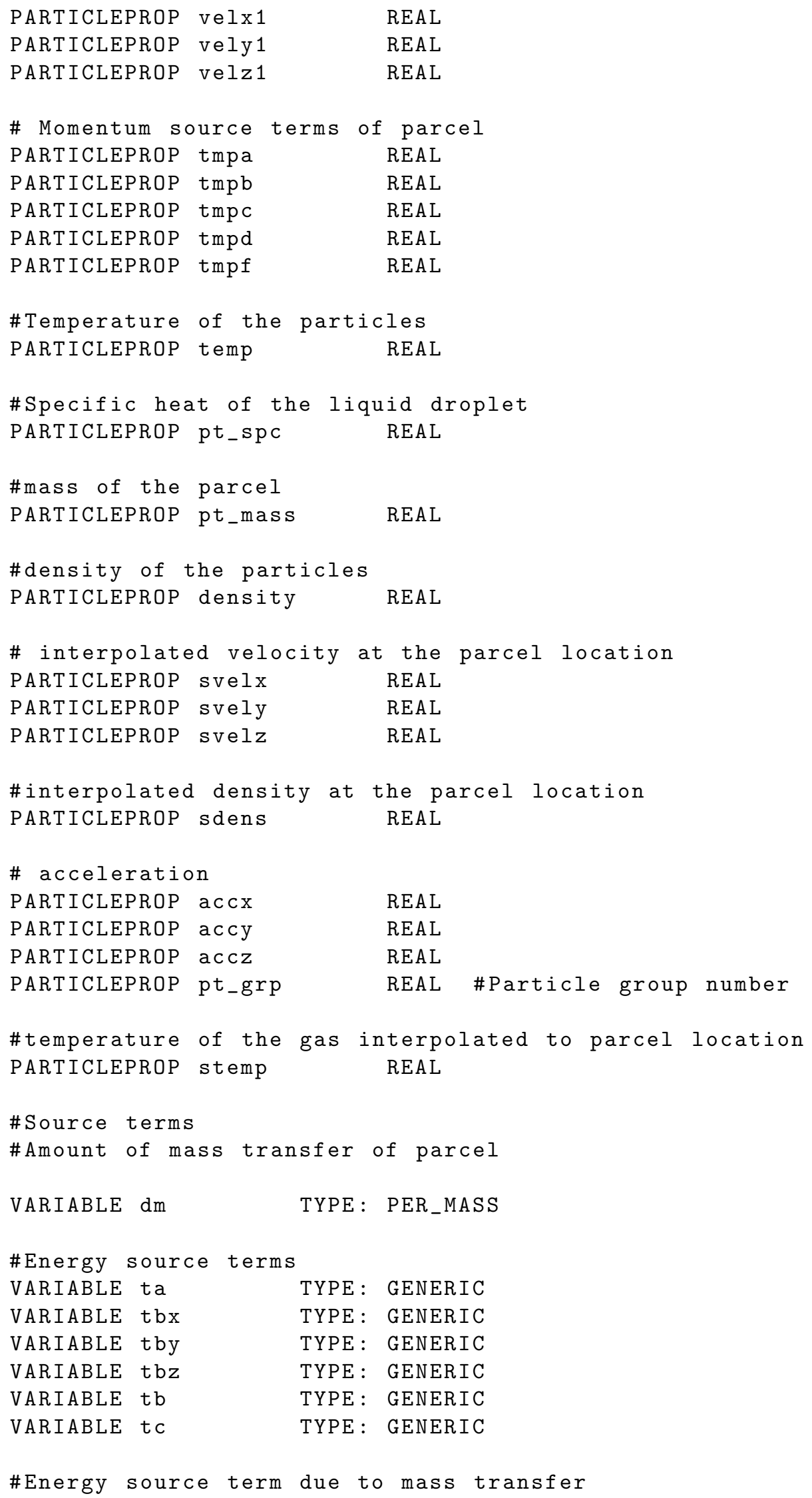




\section{A.1.2 Makefile}

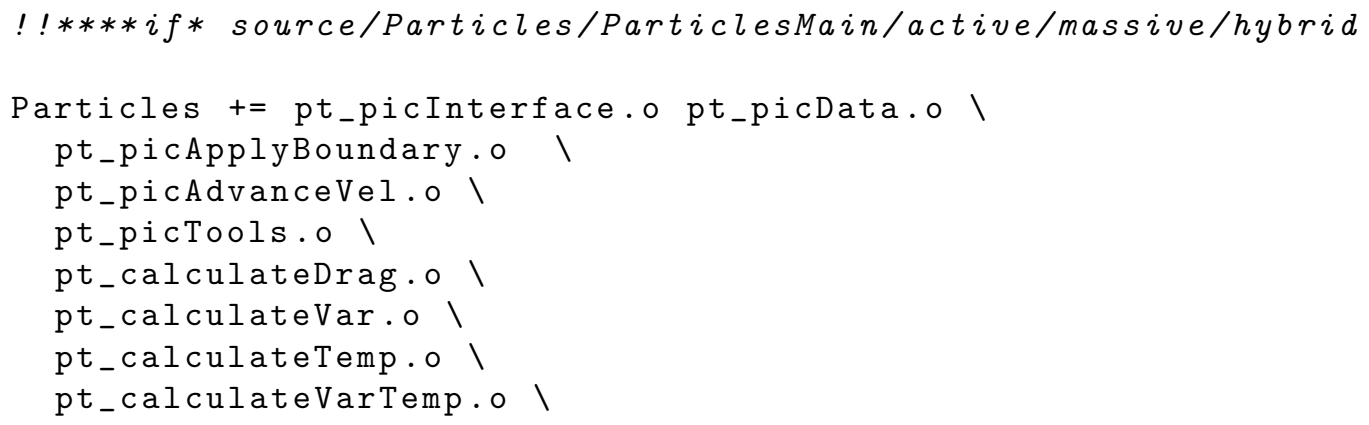

\section{A.1.3 pt_picInterface.F90}

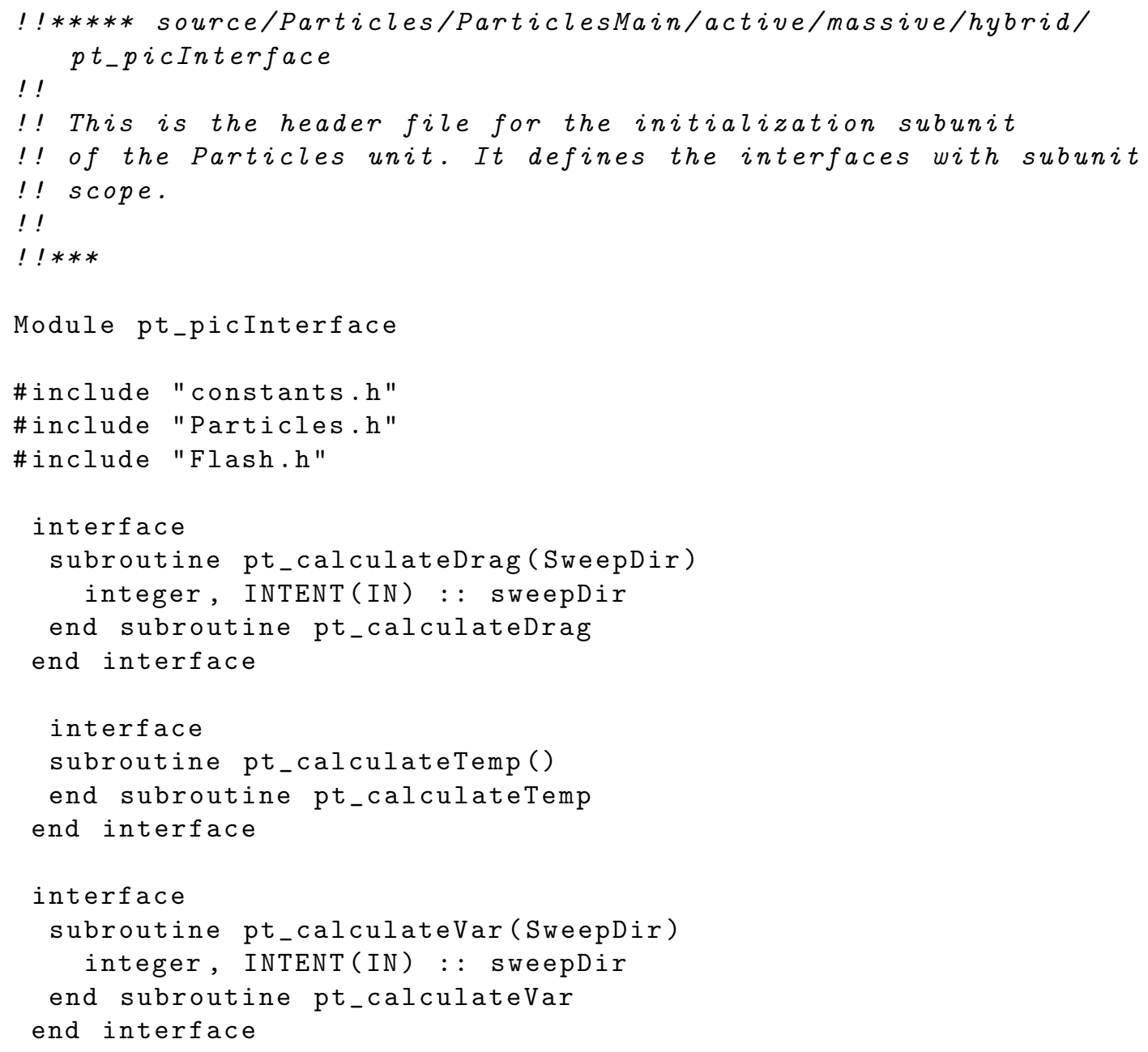




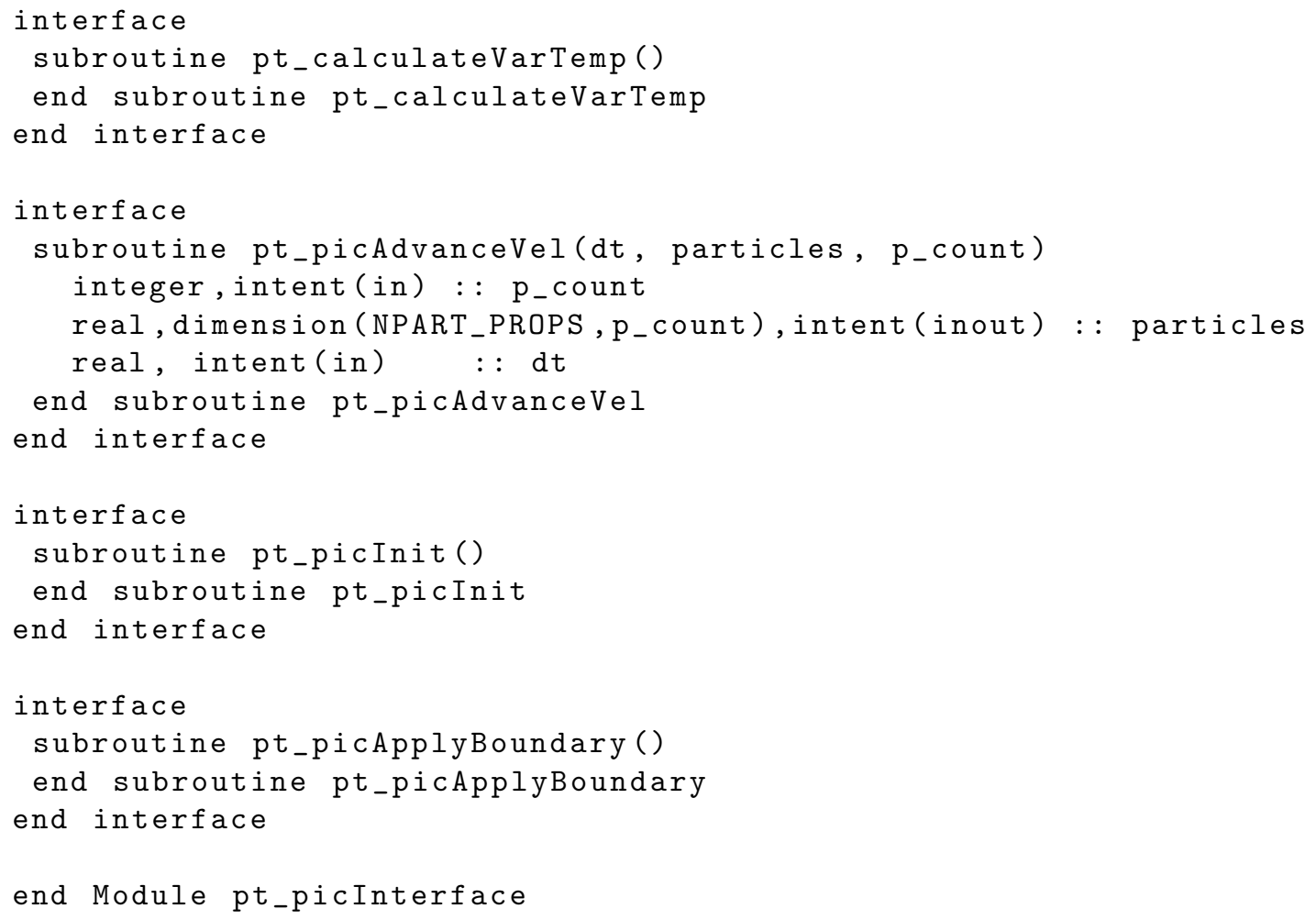

\section{A.1.4 pt_picData.F90}

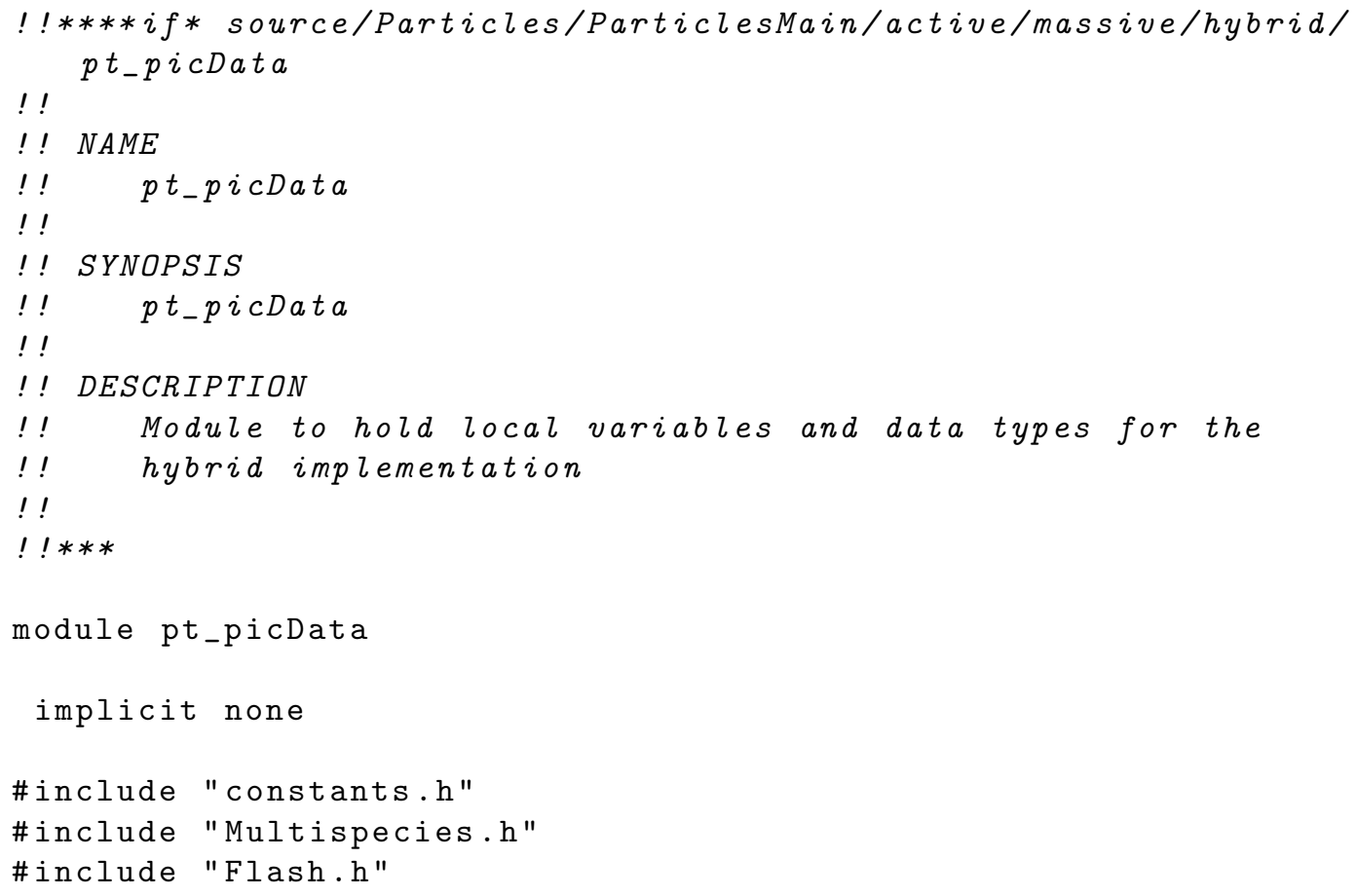




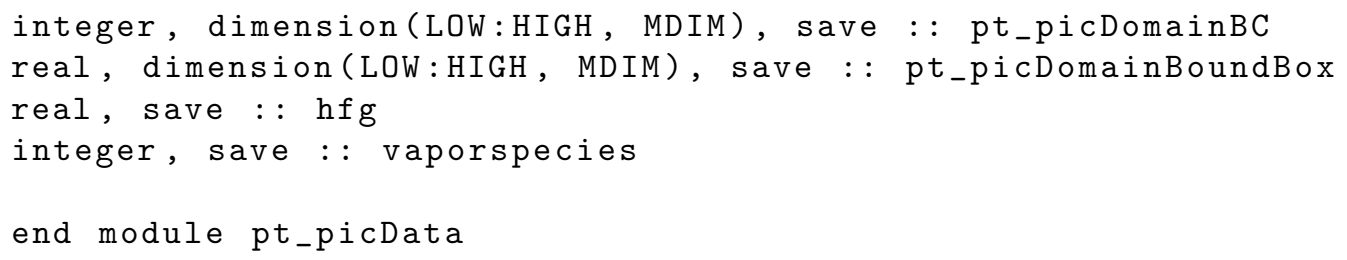

\section{A.1.5 pt_picInit.F90}

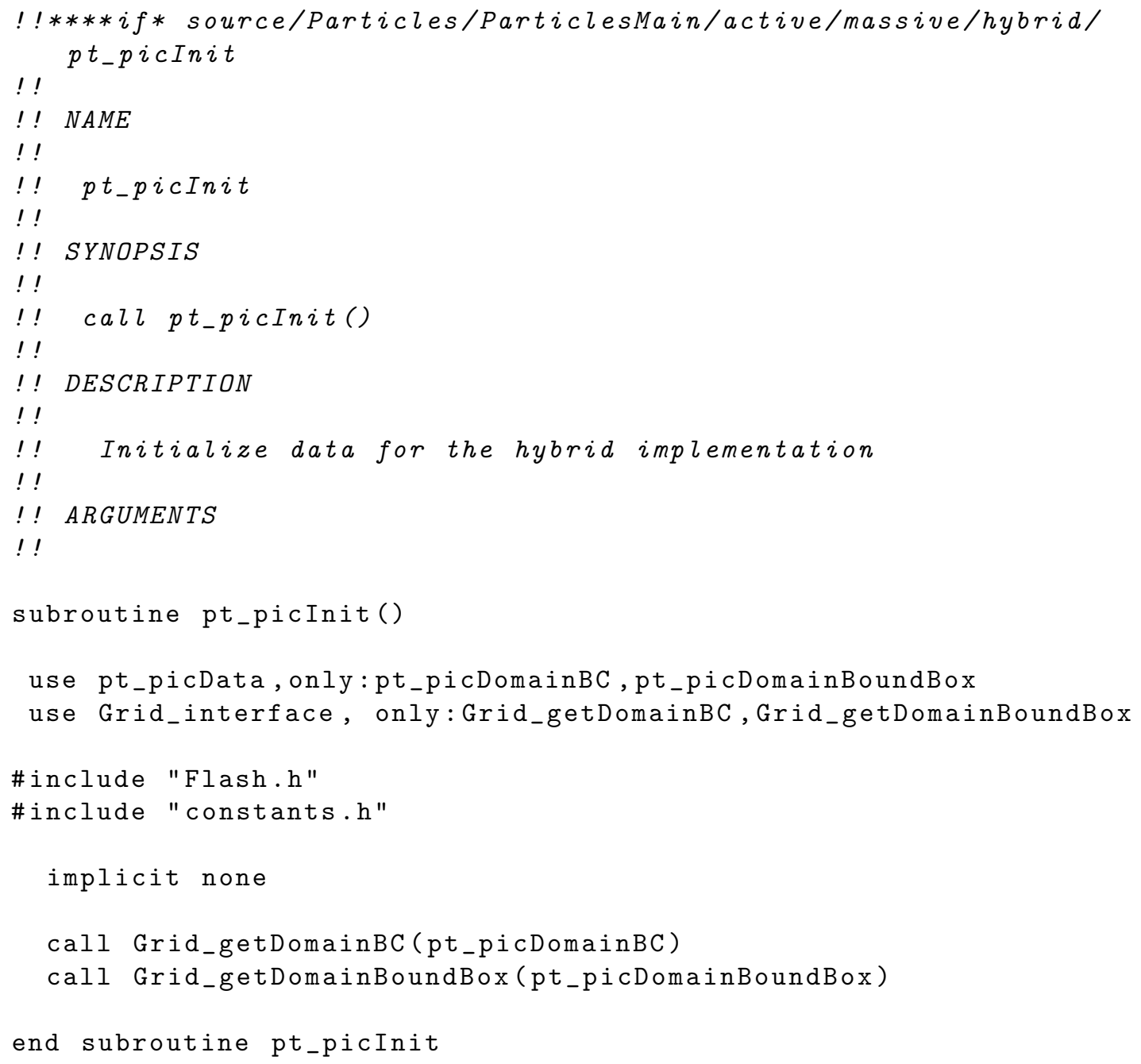

\section{A.1.6 pt_calculateDrag.F90}

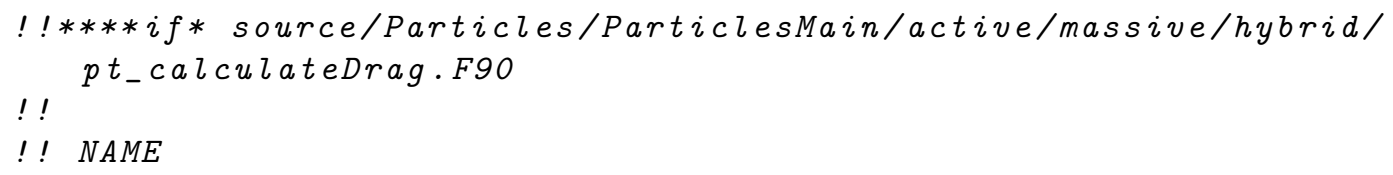




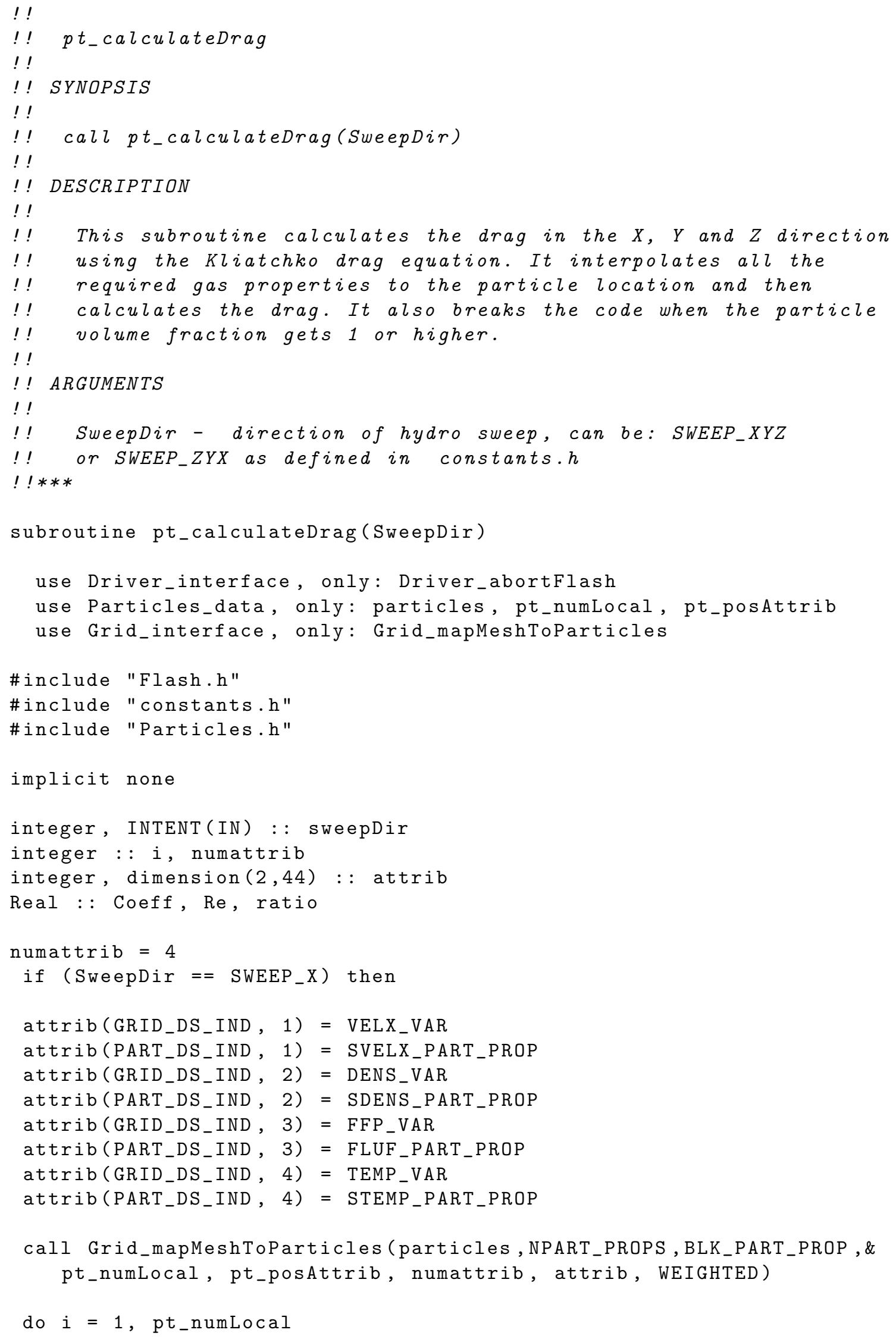




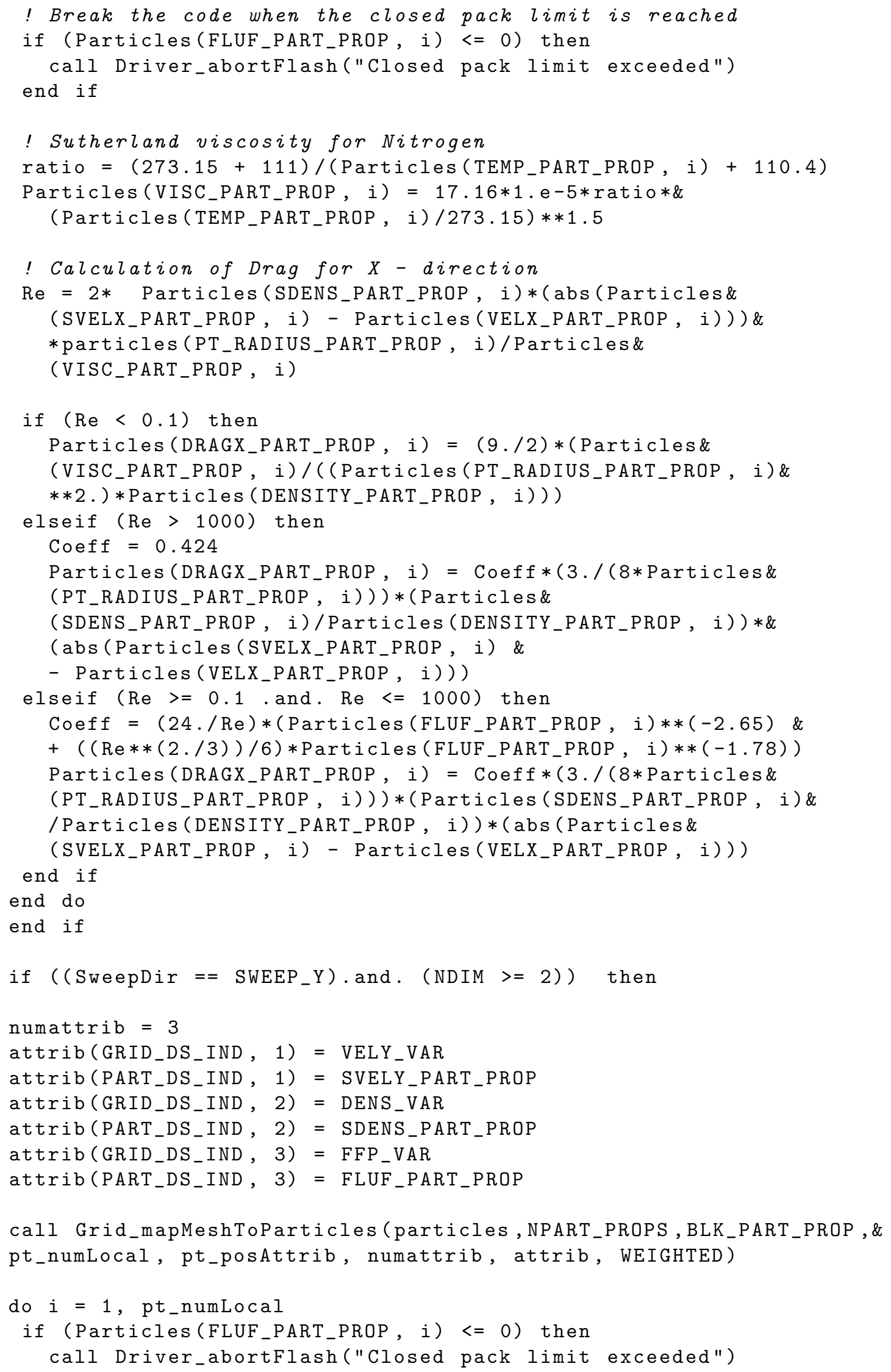




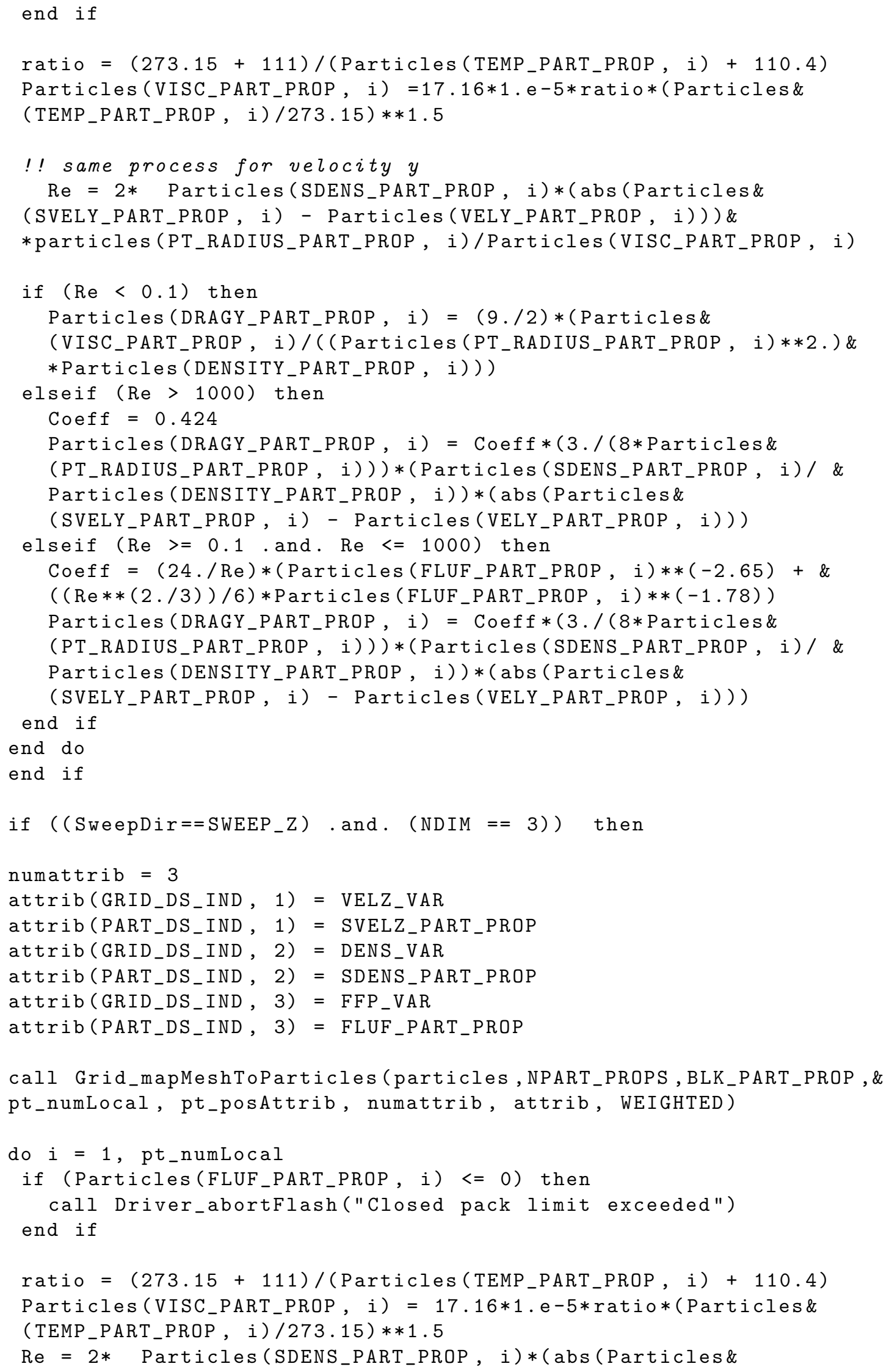




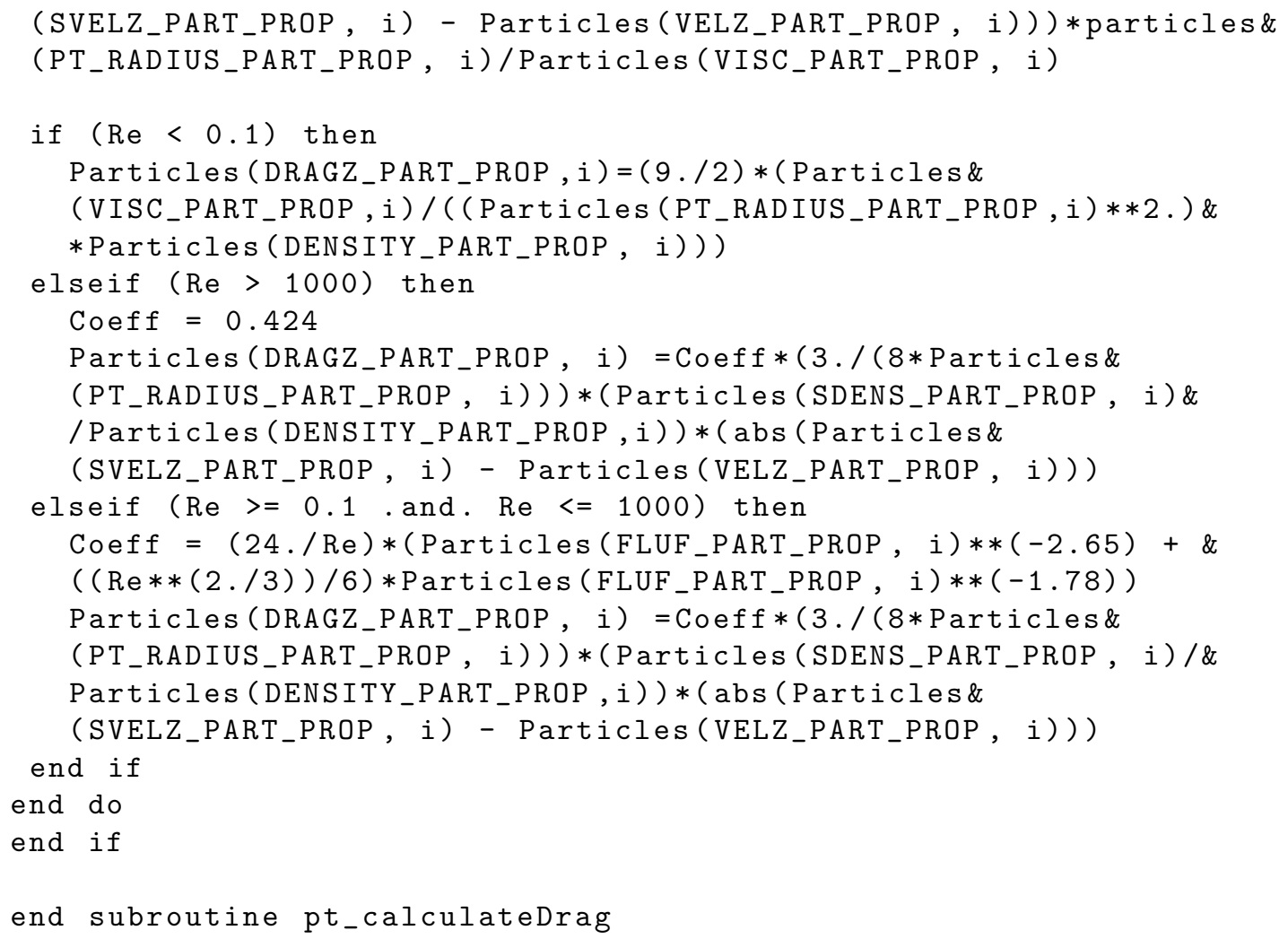

\section{A.1.7 pt_calculateVar.F90}

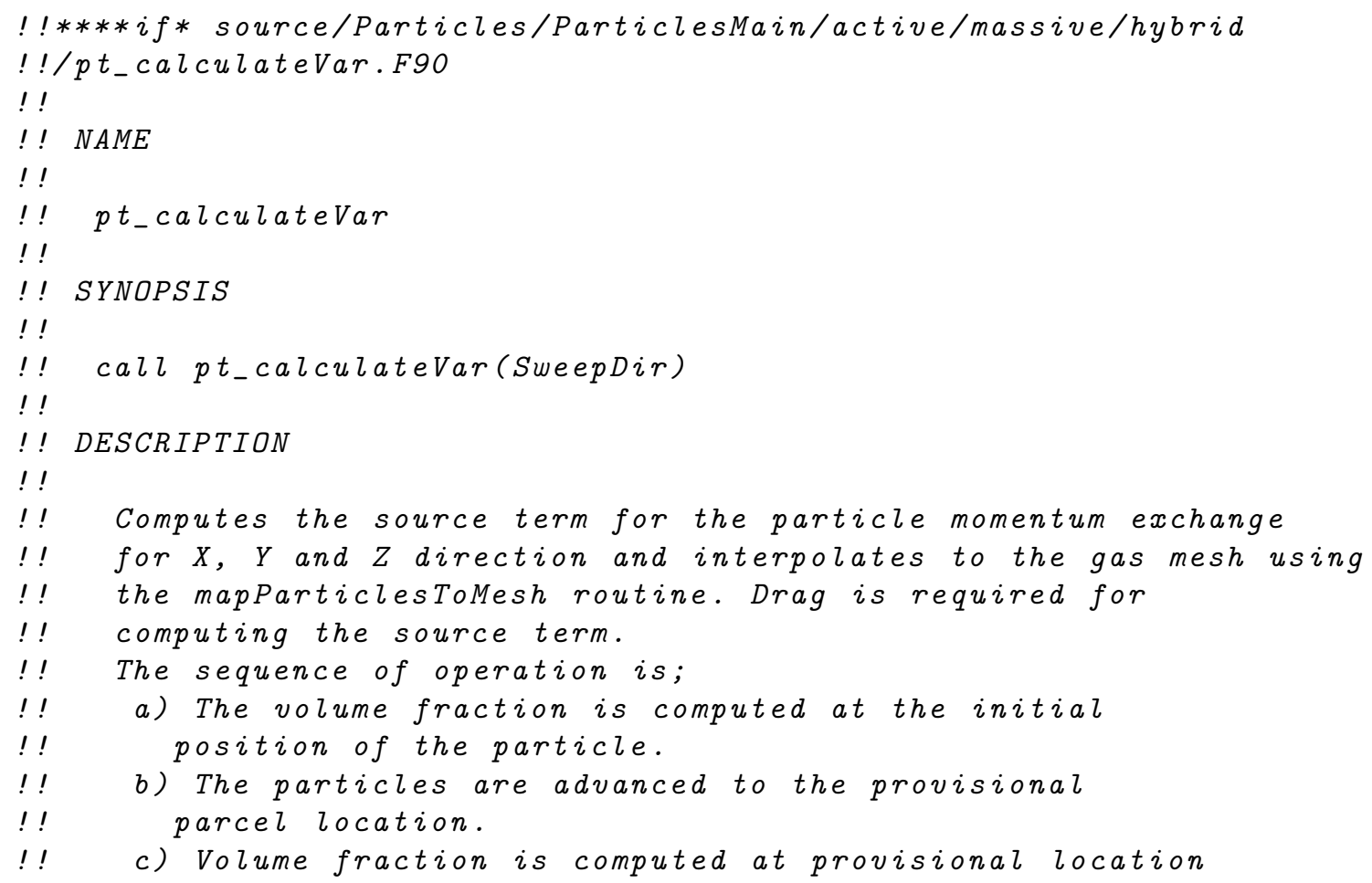




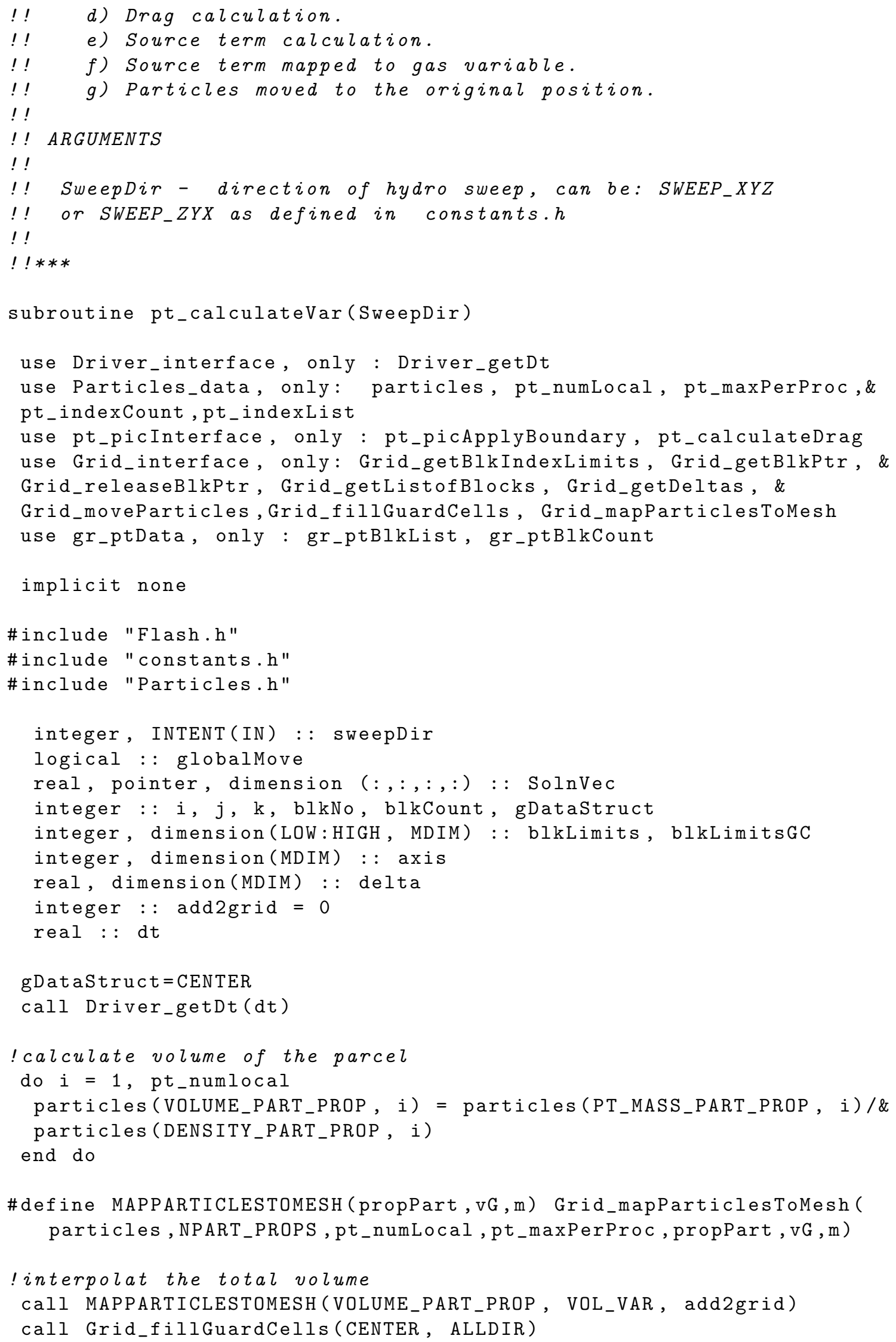




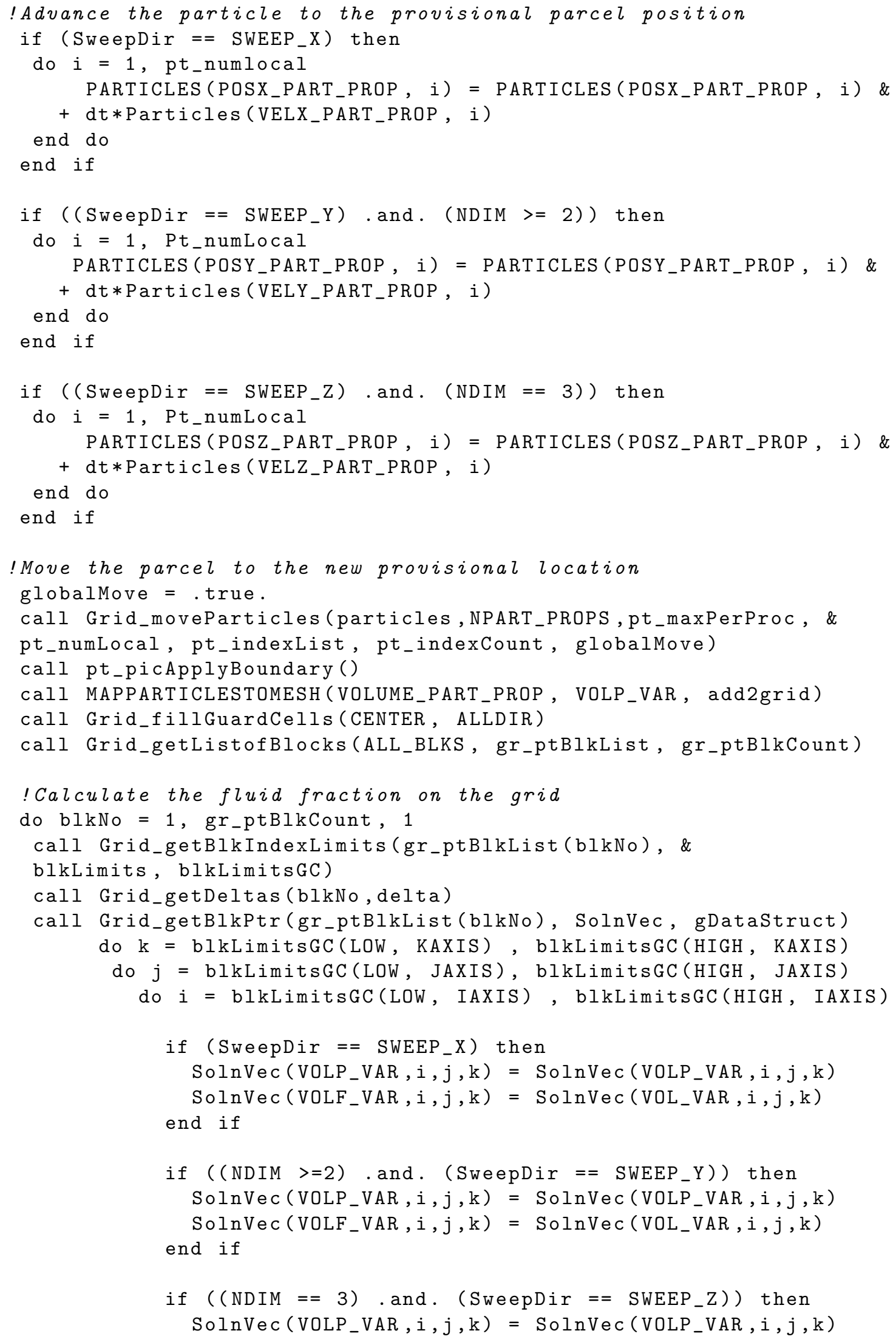




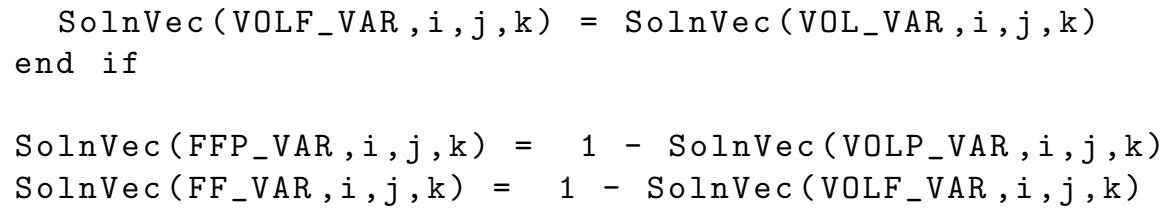




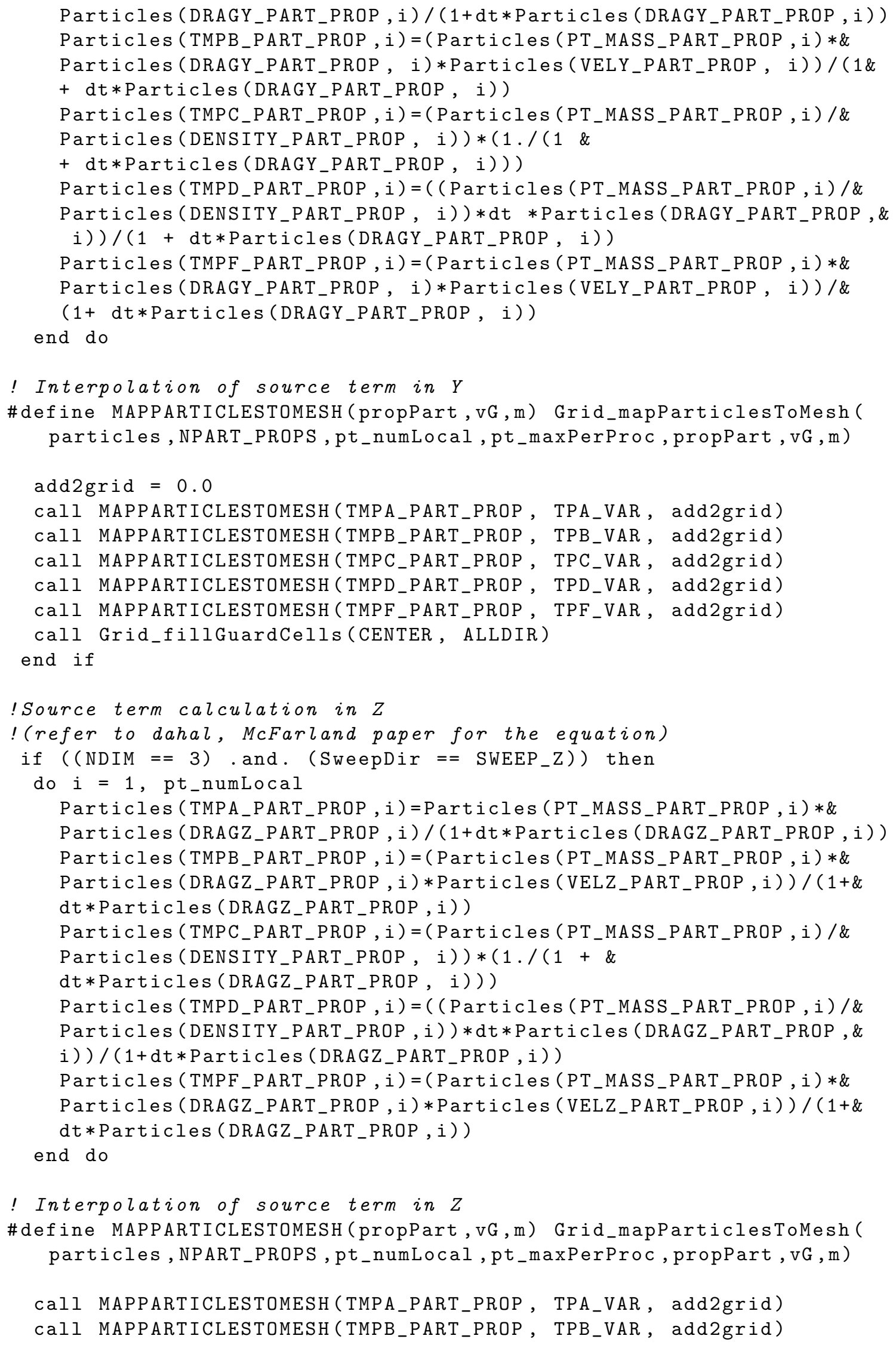




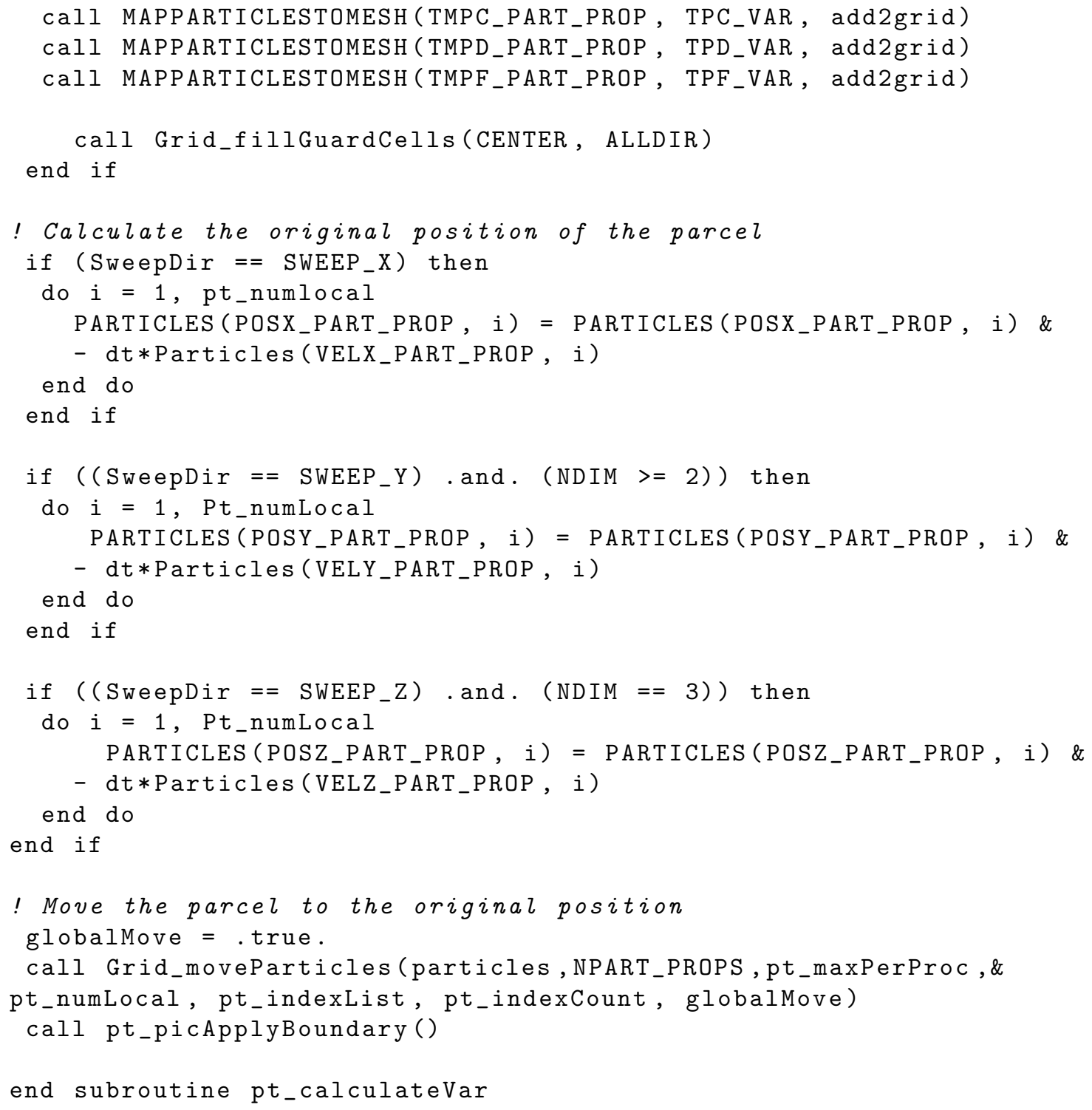

\section{A.1.8 pt_picAdvanceVel}

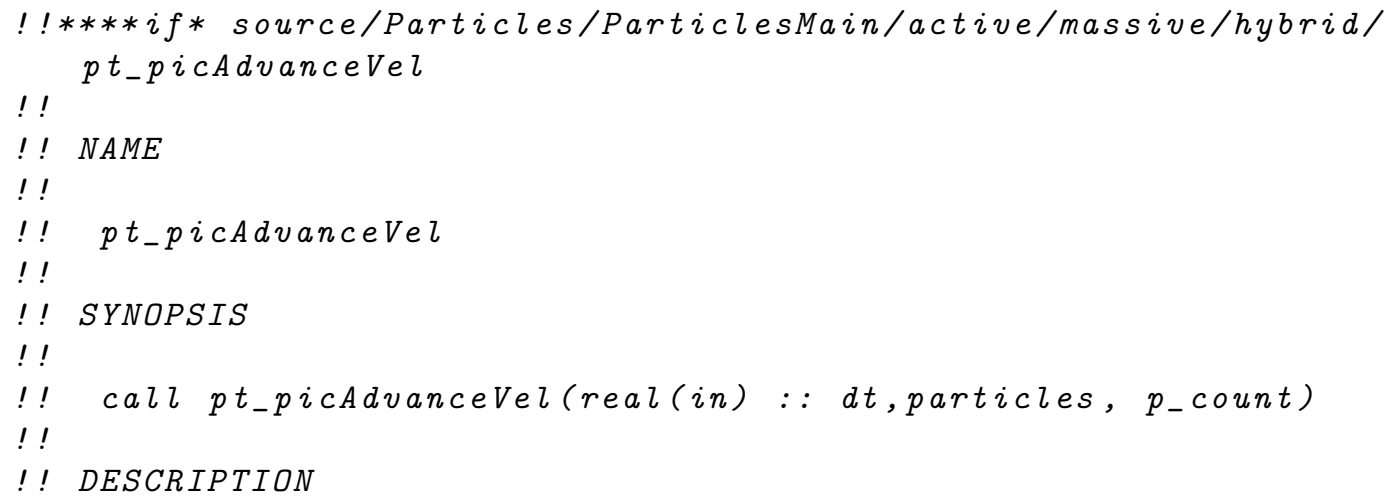




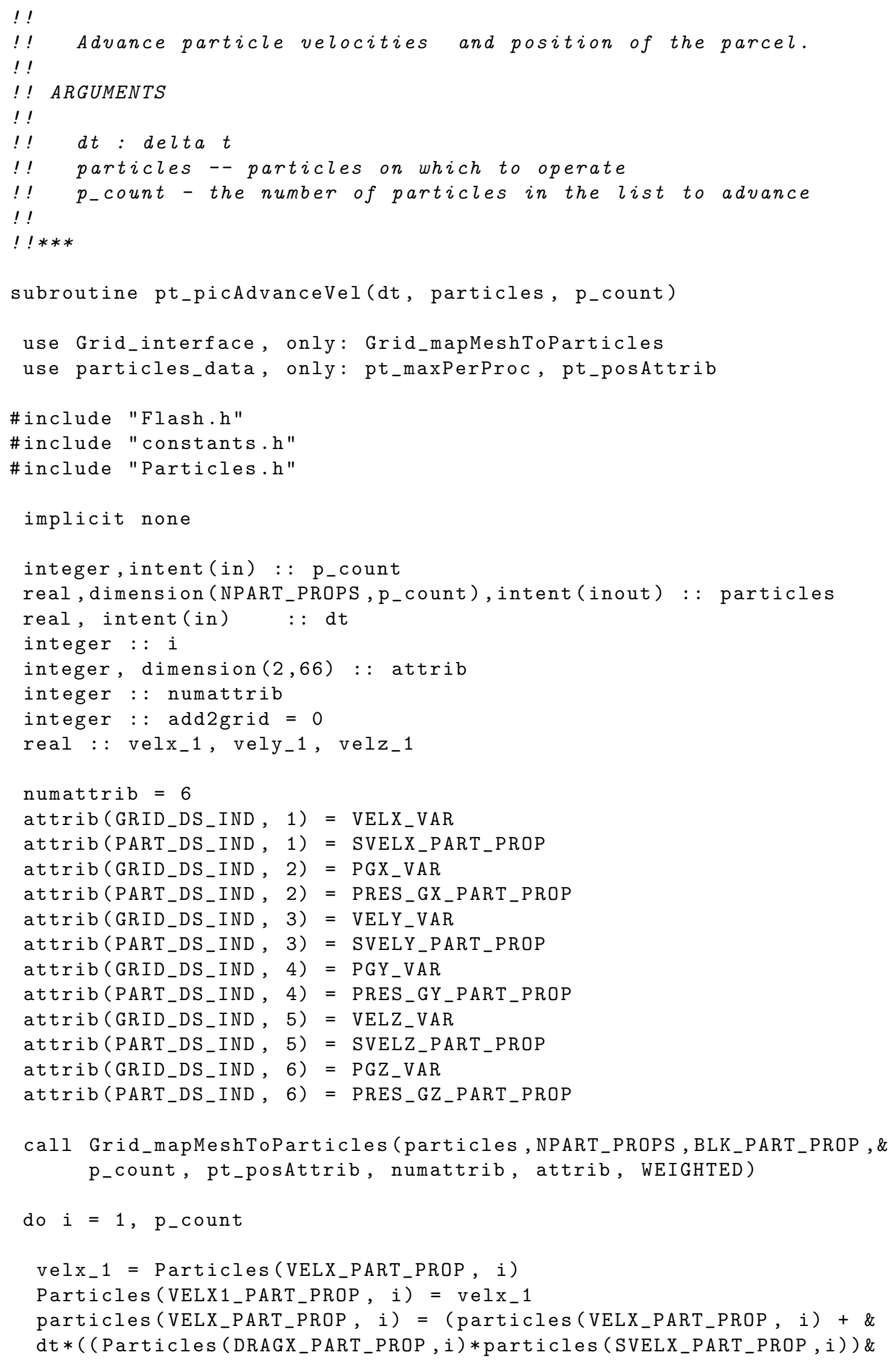




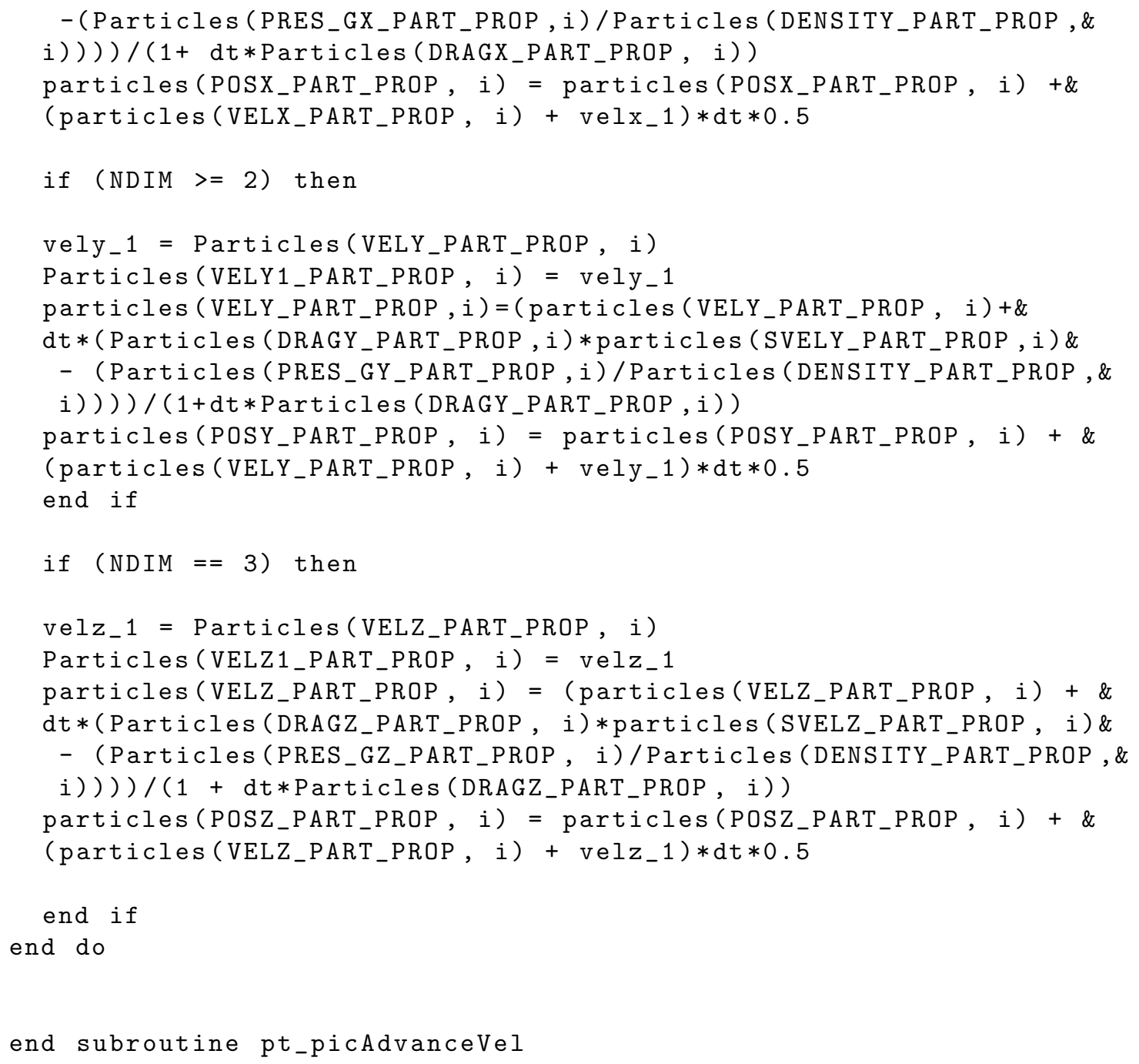

\section{A.1.9 pt_advanceCharged.F90}

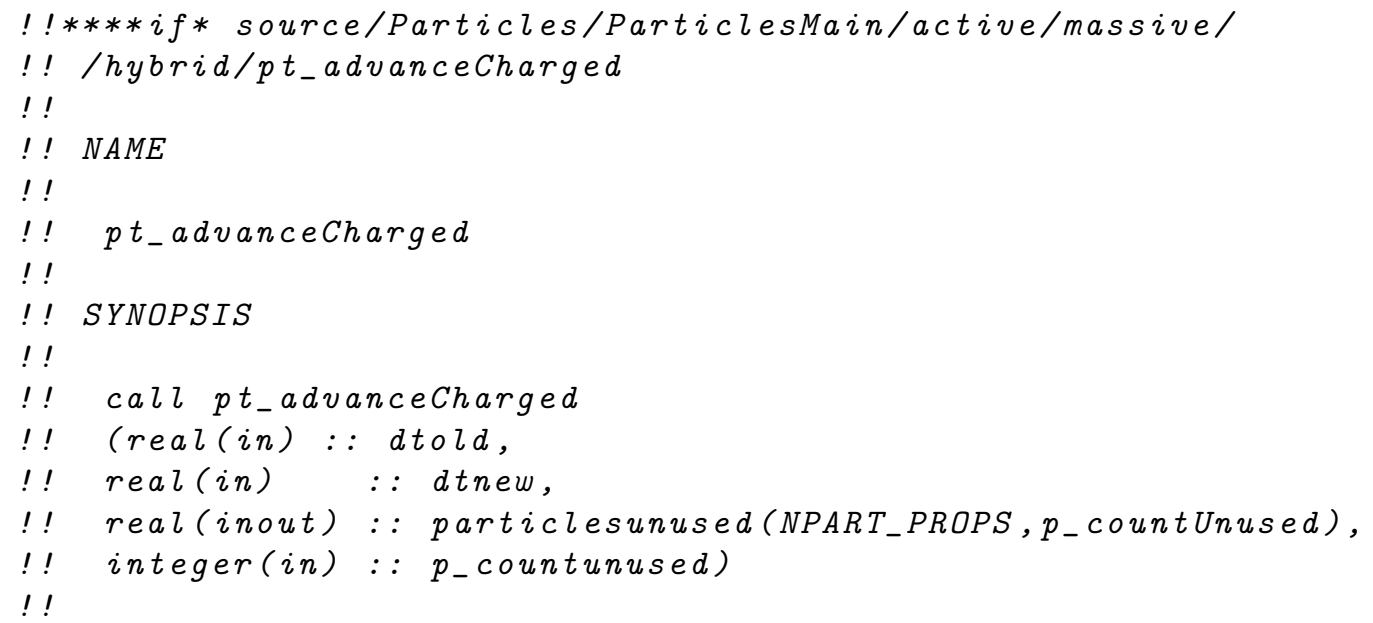




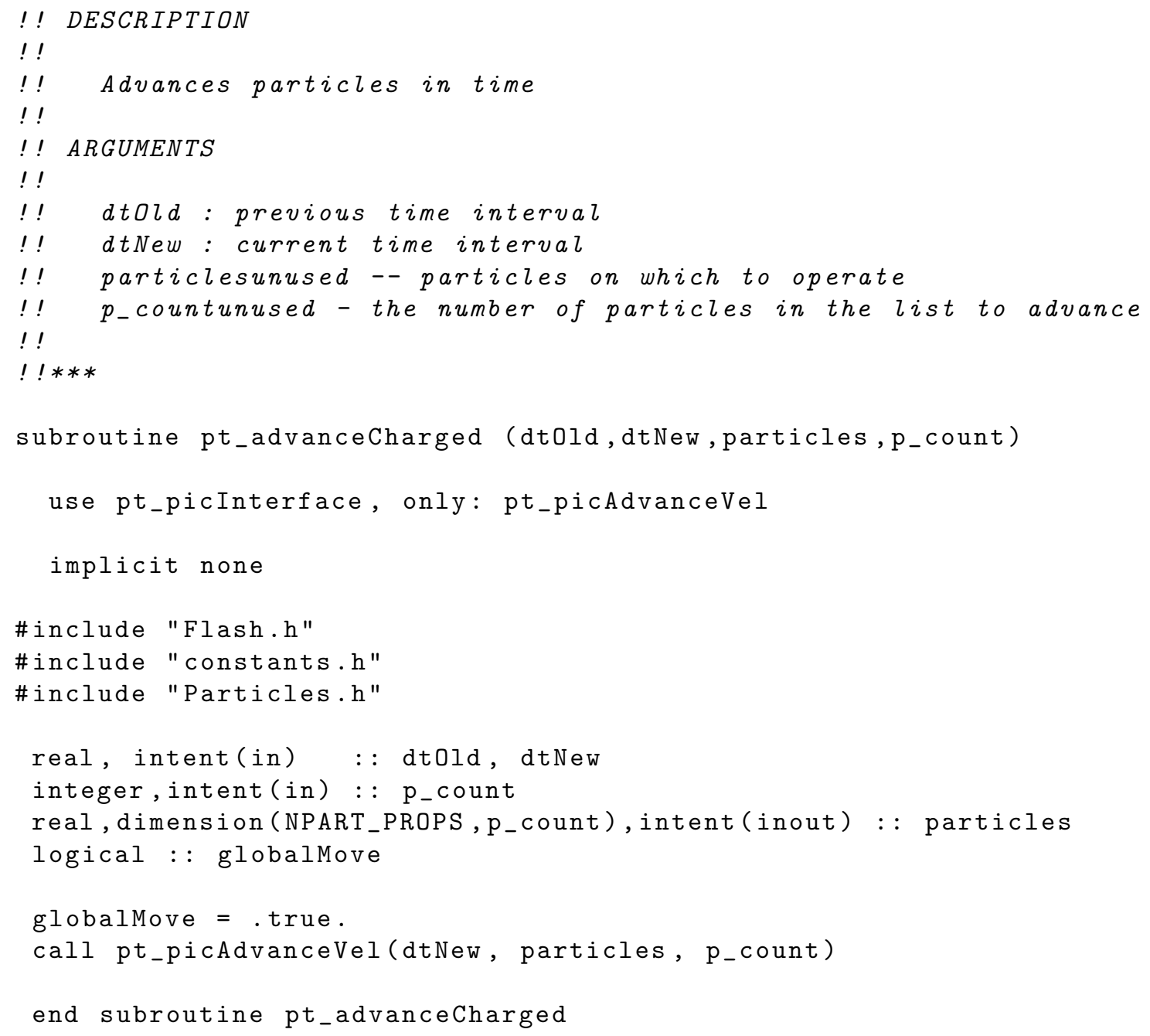

\section{A.1.10 pt_calculateVarTemp}

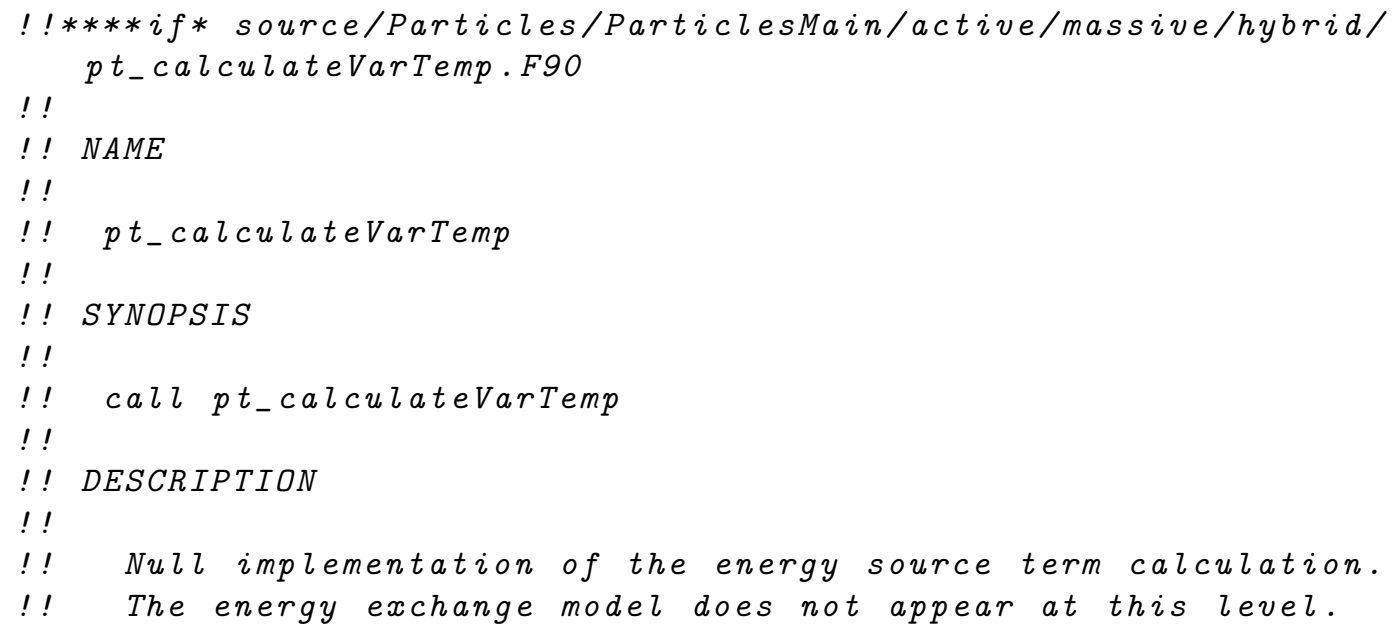




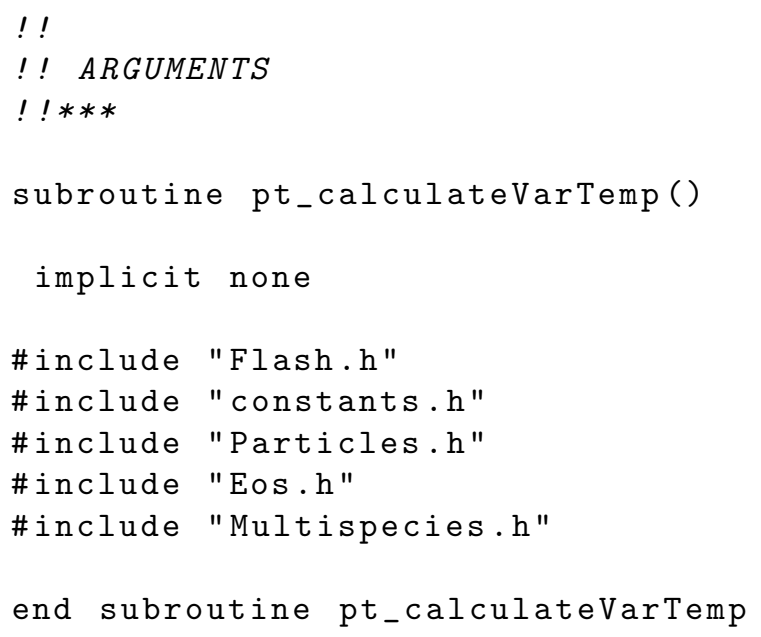

\section{A.1.11 pt_calculateTemp}

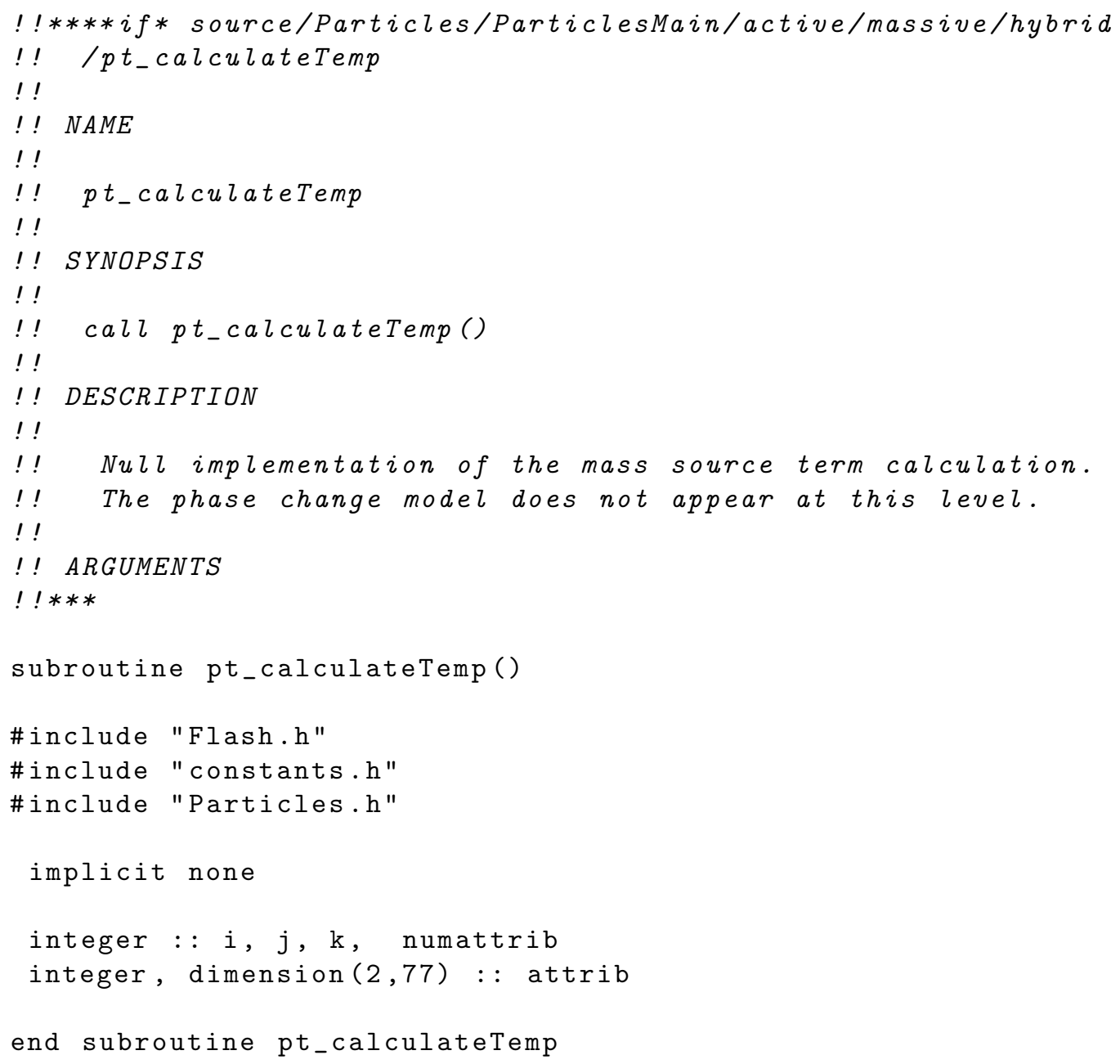




\section{A.2 Energy Exchange}

The energyexchange model that we built in the FLASH code is located at source $\backslash$ Simulation $\backslash$ Simulat

The contents of the directory are;

- phasechange (directory containing phase change model files)

- Config

- Makefile

- pt_picData.F90

- pt_picInit.F90

- pt_calculateVarTemp.F90

- pt_calculateTemp.F90

- pt_advanceCharged.F90

The detail subroutines are included below.

\section{A.2.1 Config}

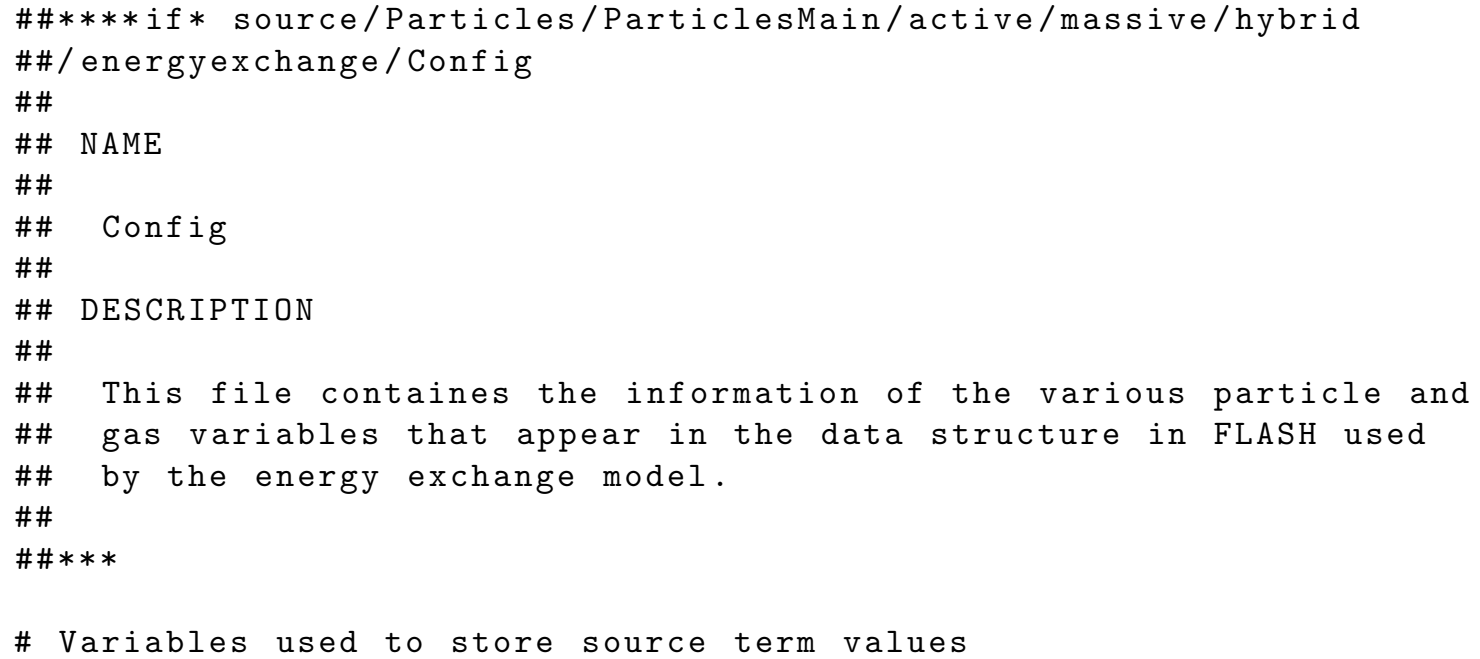




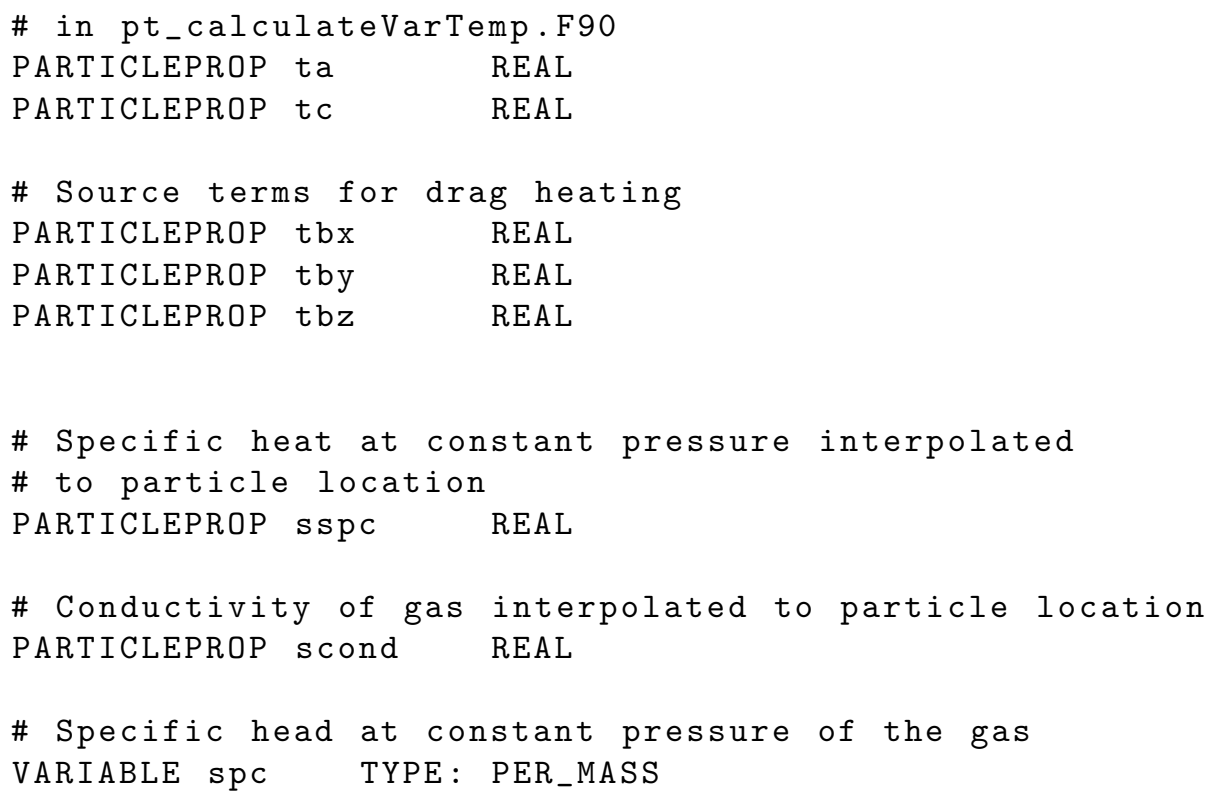

\section{A.2.2 Makefile}

Particles $+=$ pt_calculateVarTemp.o \

pt_calculateTemp.o

\section{A.2.3 pt_picData}

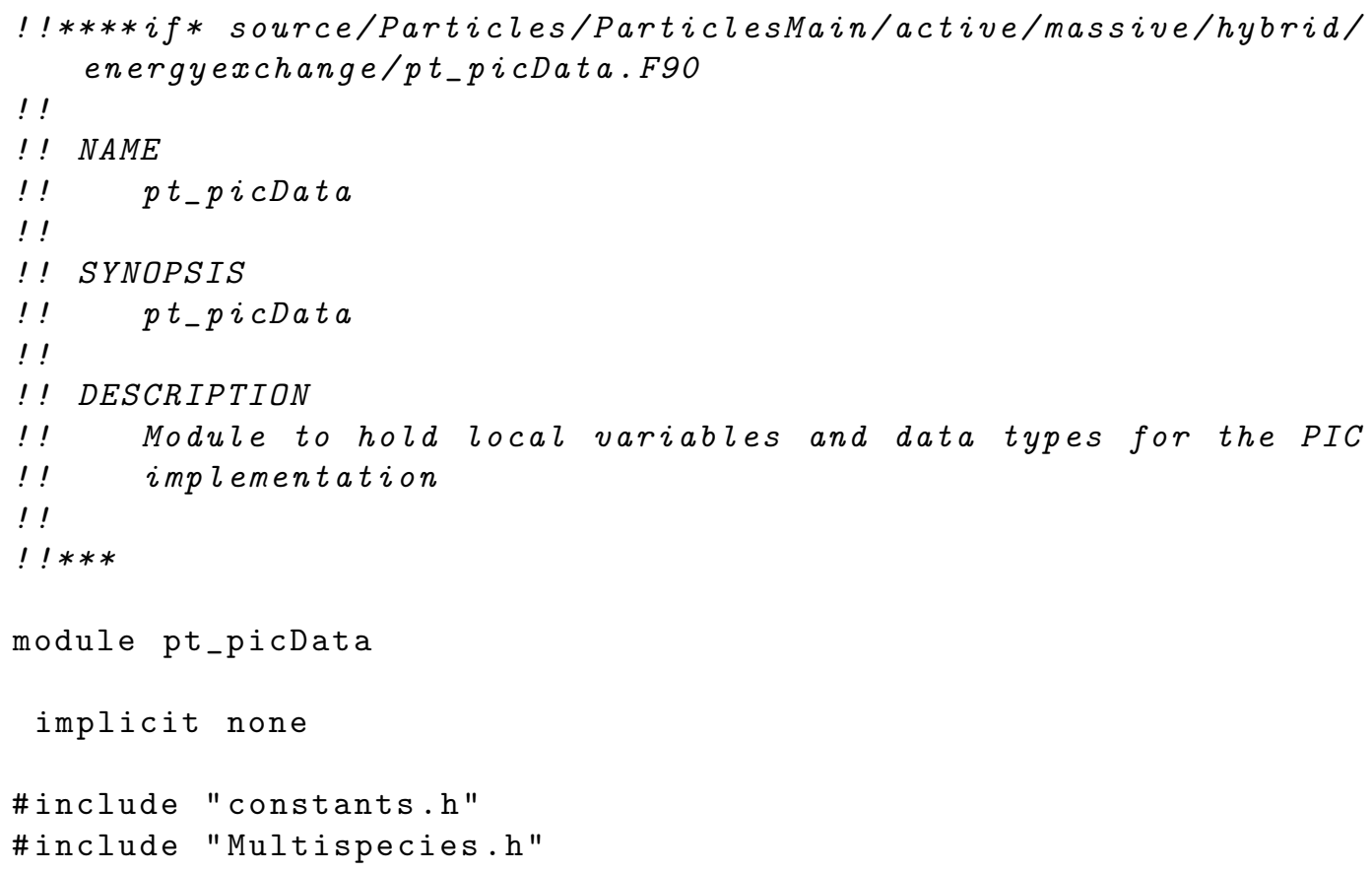




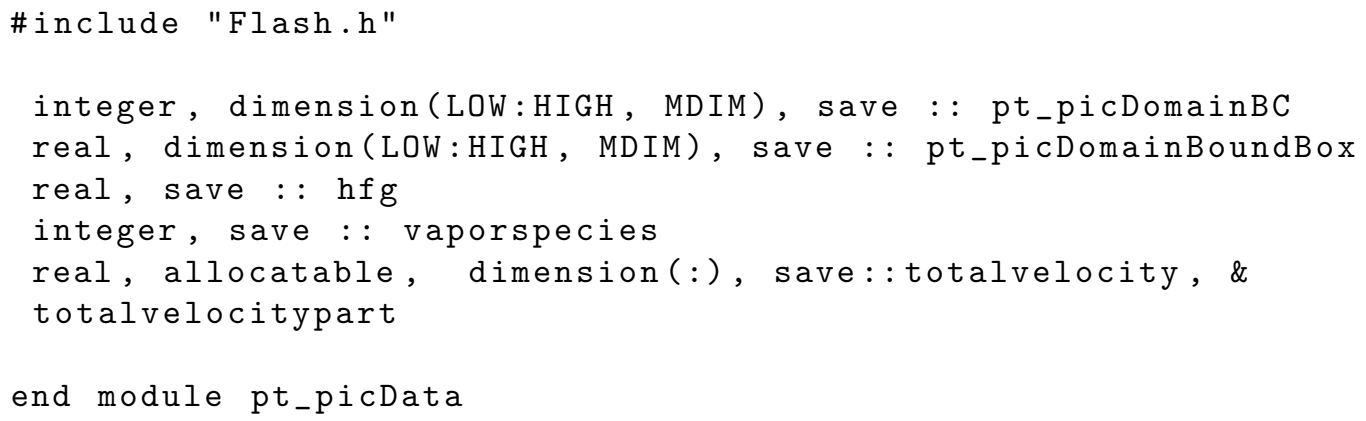

\section{A.2.4 pt_picInit}

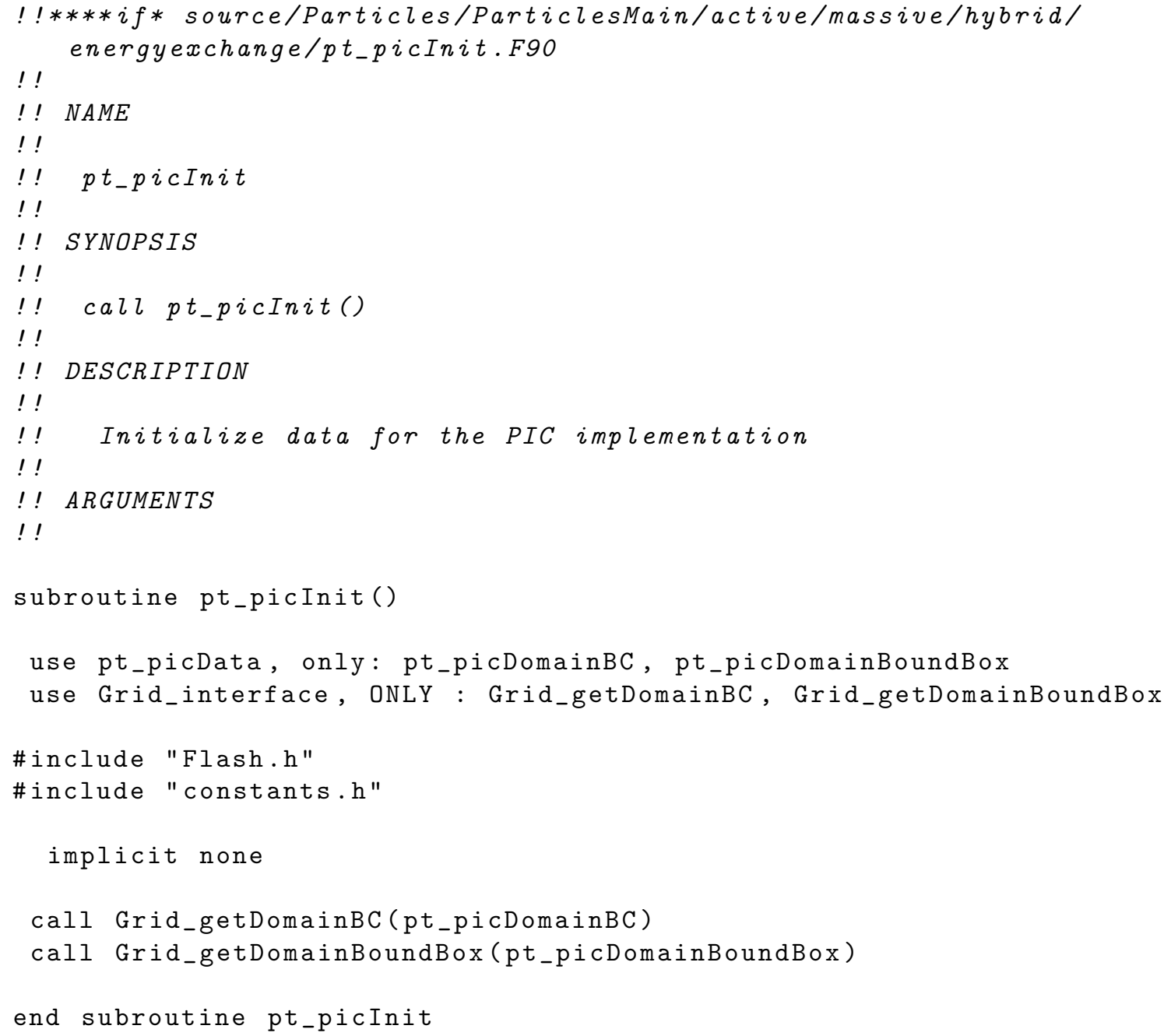

\section{A.2.5 pt_calculateVarTemp.F90}

!!****if* source/Particles/ParticlesMain/active/massive/hybrid/ 
energyexchange/pt_calculateVarTemp. F90

!! Computes the source term for the particle energychange

!! and interpolates to the gas mesh using the mapParticlesToMesh

!! routine.

!! The sequence of operation is;

$! !$

a) The particles are advanced to the provisional parcel location.

b) Compute the specific heat at constant pressure of

!! gas using EOS call.

!! c) Volume fraction is computed at provisional location

!! d) Make calculation of the parameters like Re, Pr

!! used for solving energy equation.

!! e) Source terms calculations.

!! f) Source terms mapped to gas variable.

!! g) Particles moved to the original position.

$! !$

$! !$

!! ARGUMENTS

$! !$

$! ! * * *$

subroutine pt_calculateVarTemp()

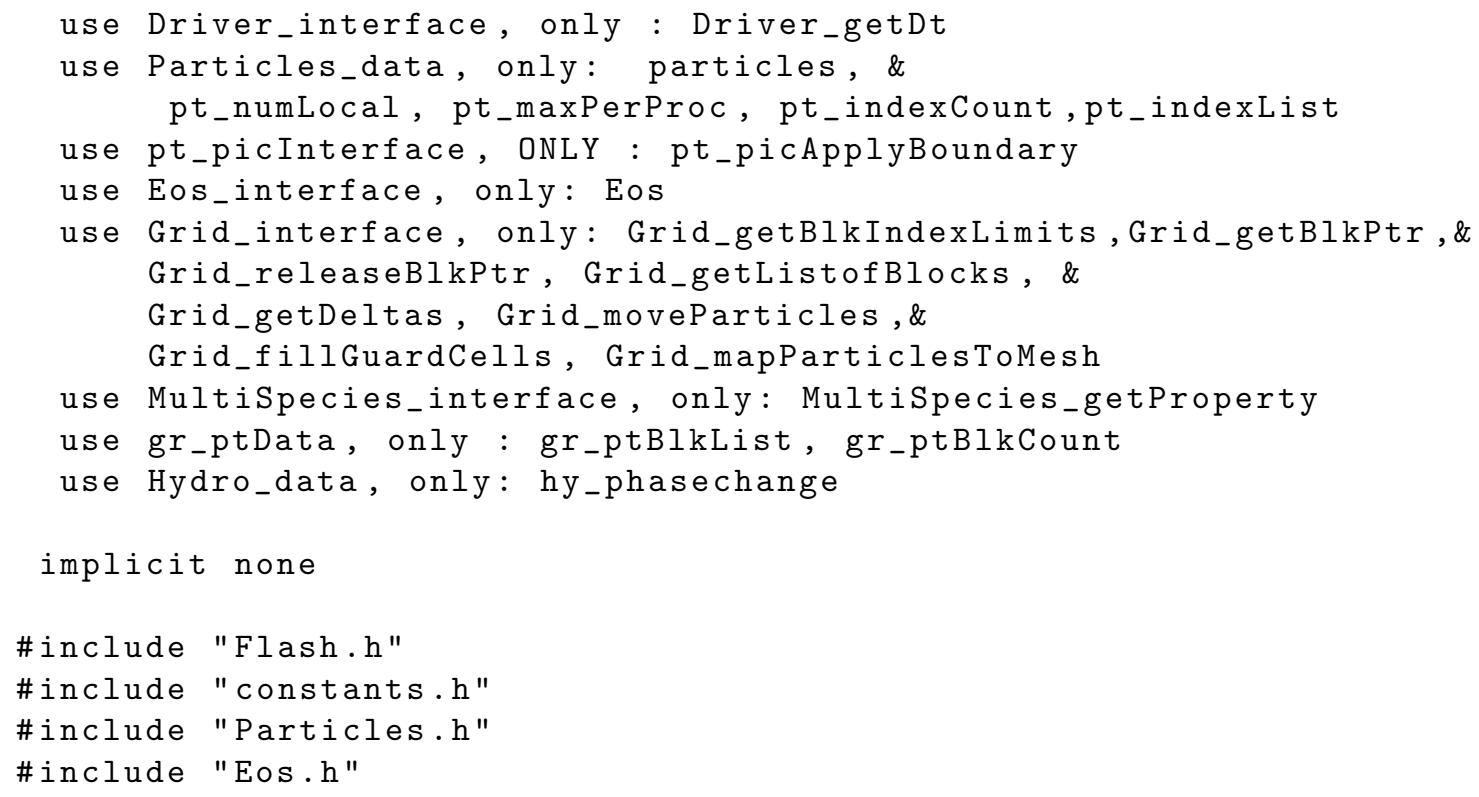




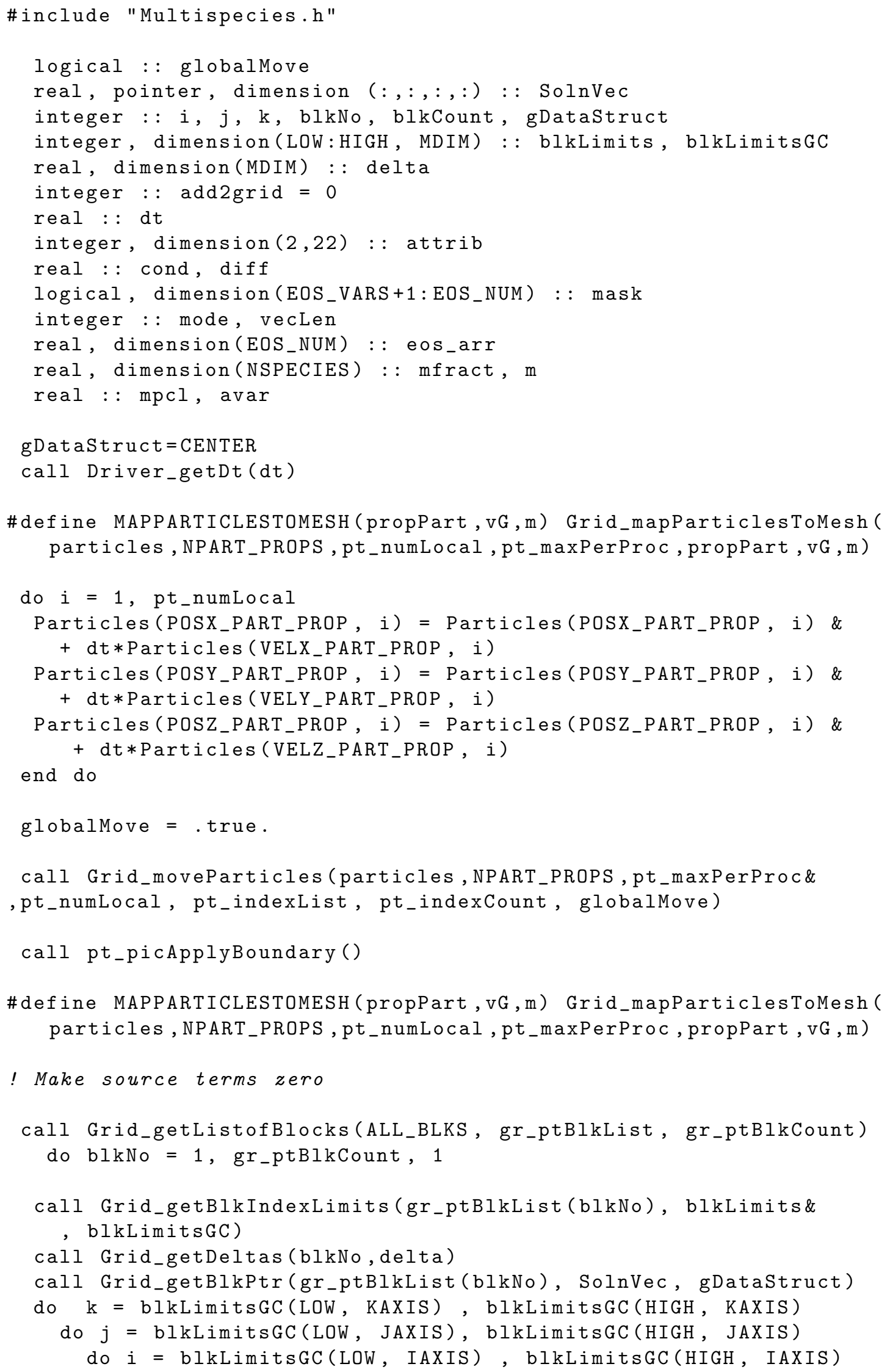




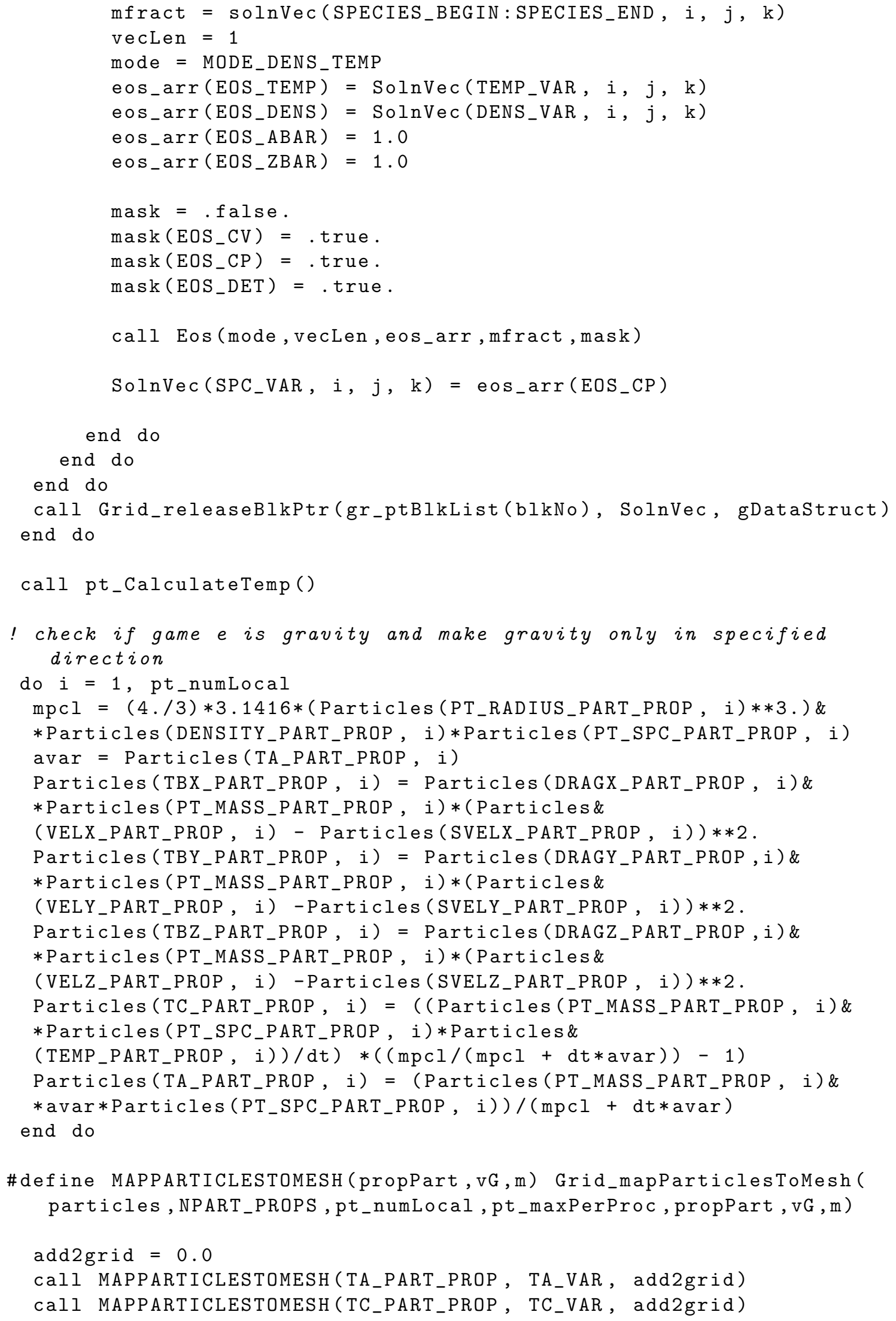




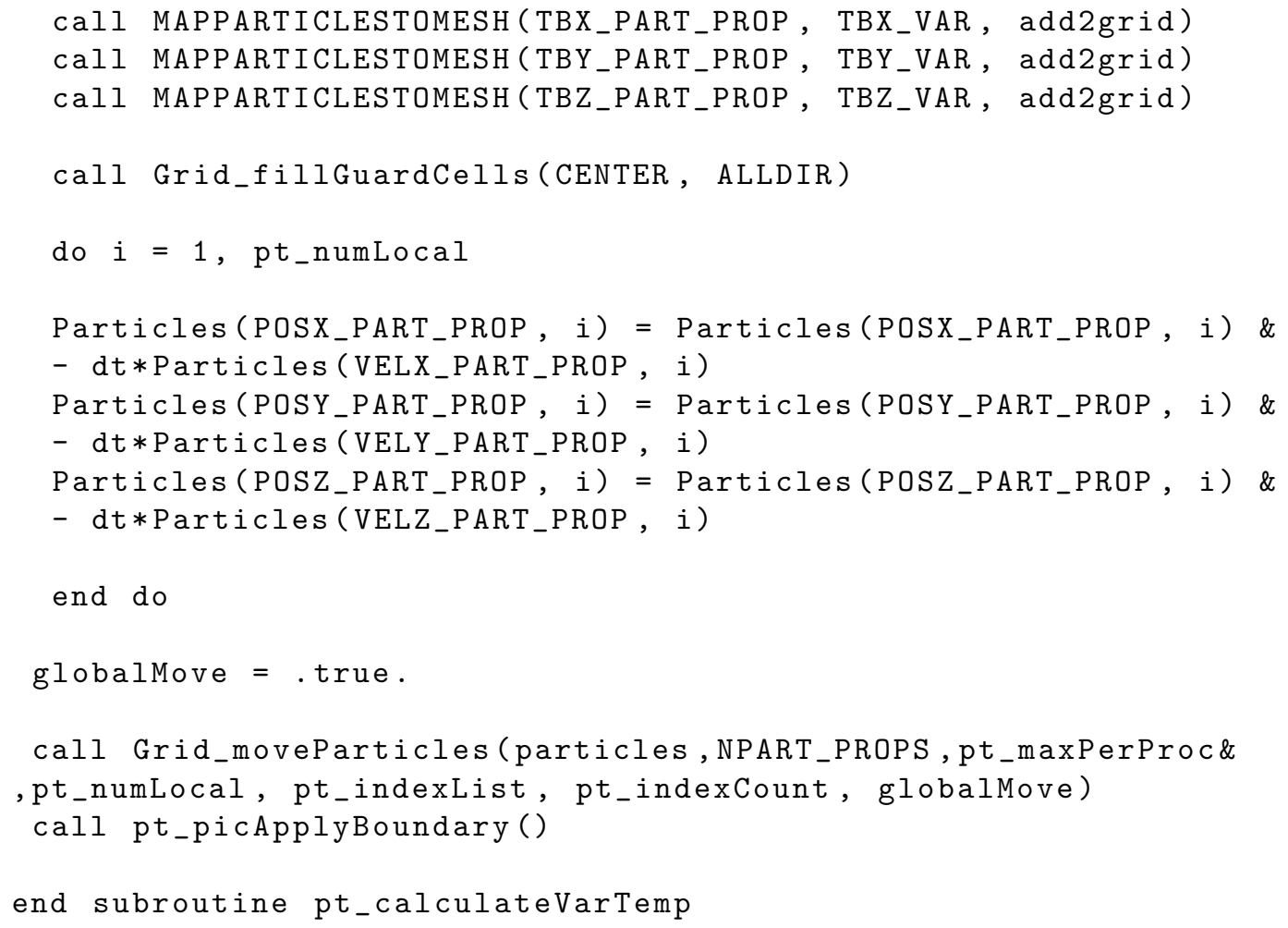

\section{A.2.6 pt_calculateTemp.F90}

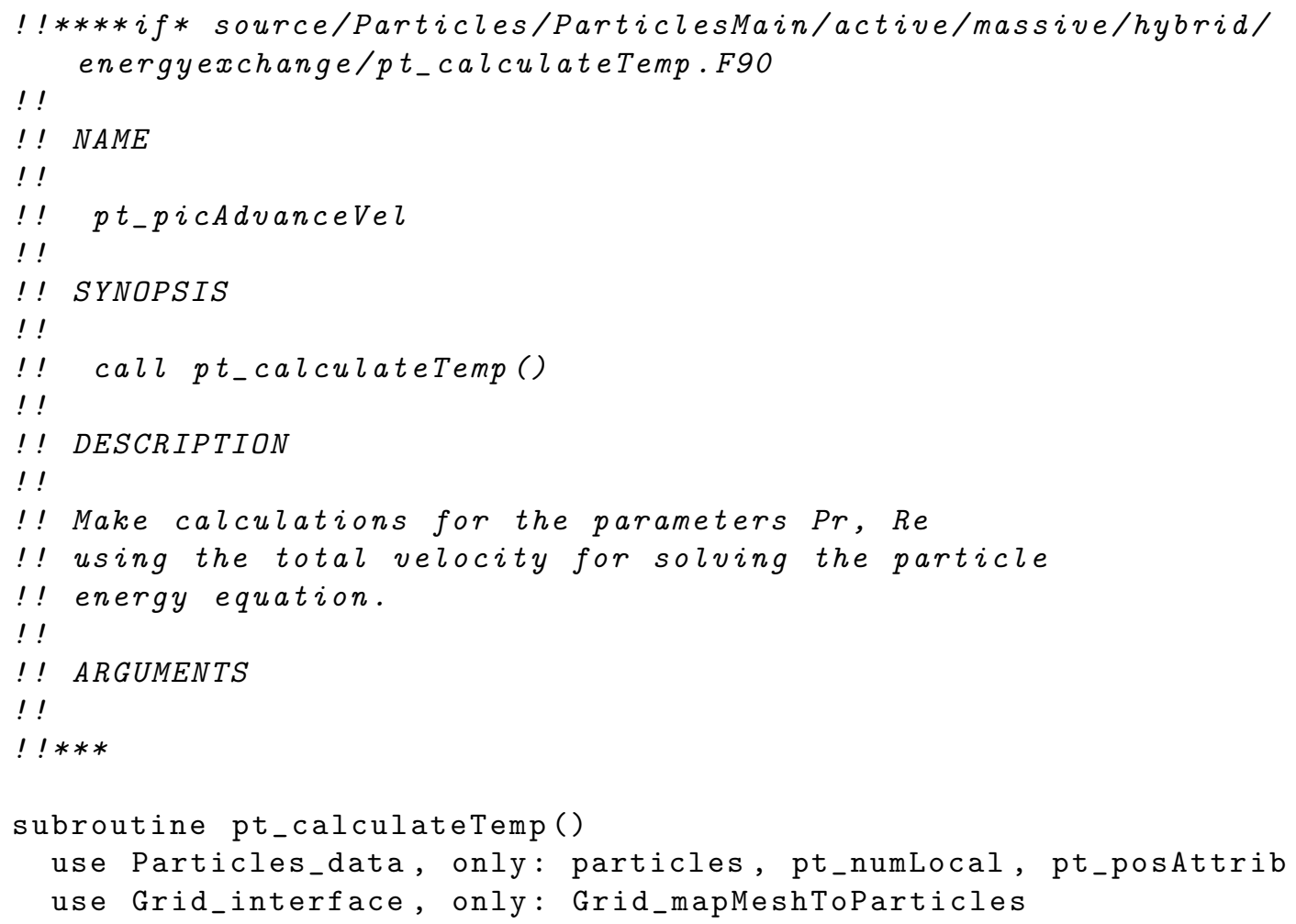




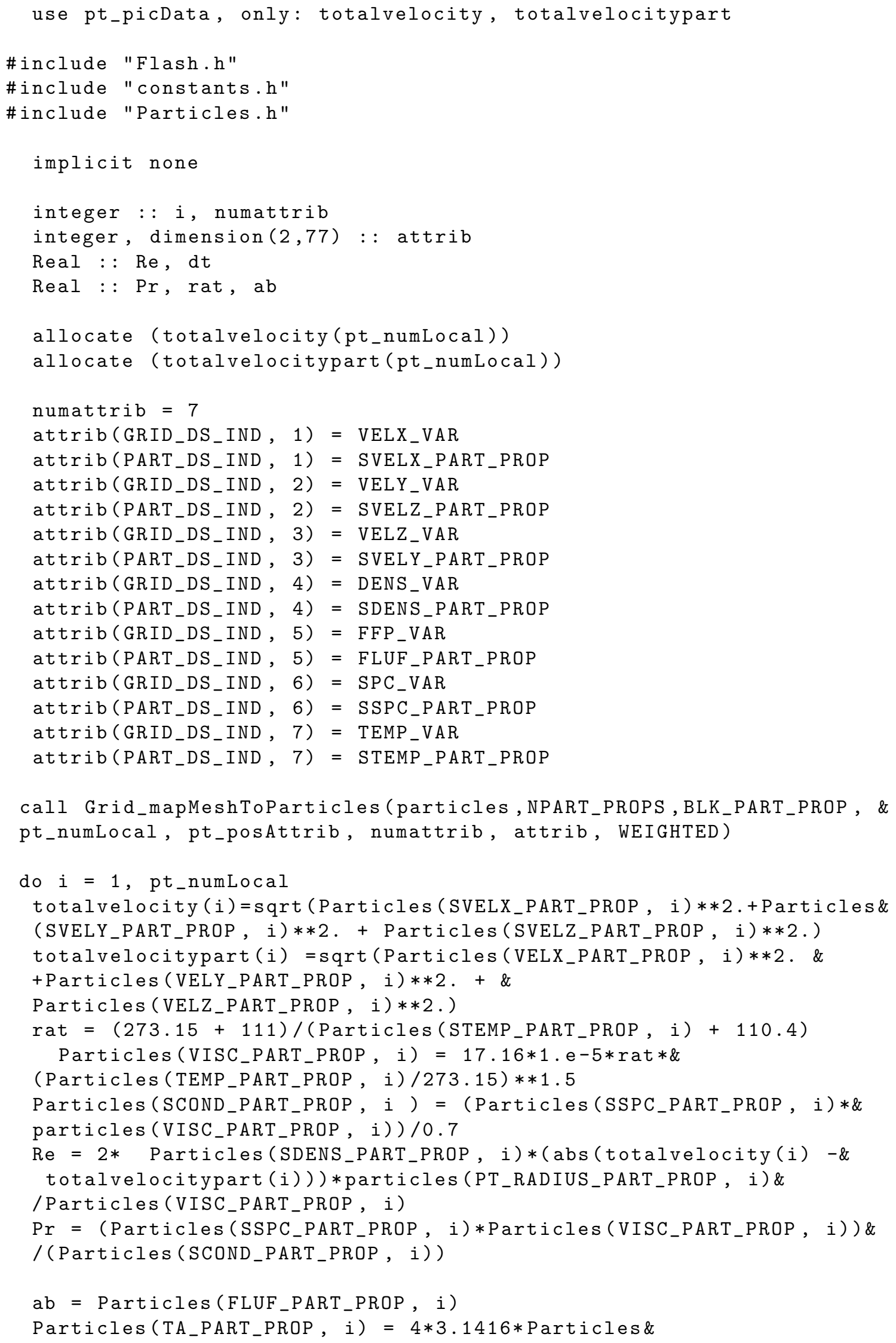




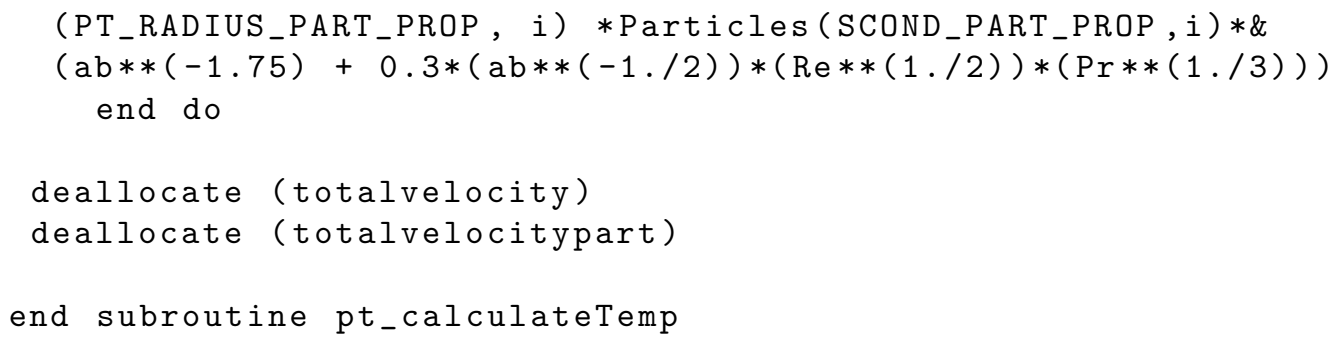

\section{A.2.7 pt_advanceCharged.F90}

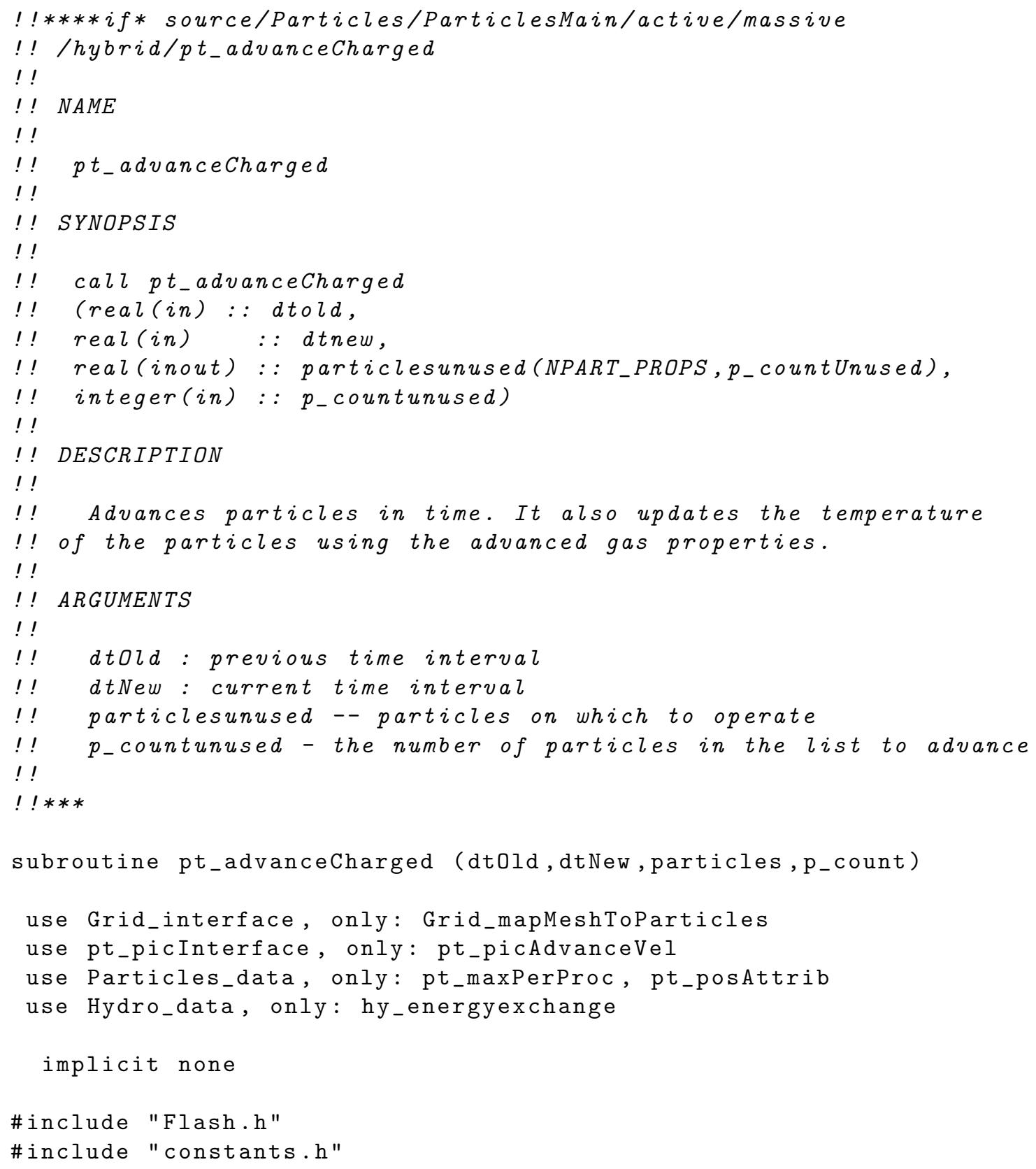




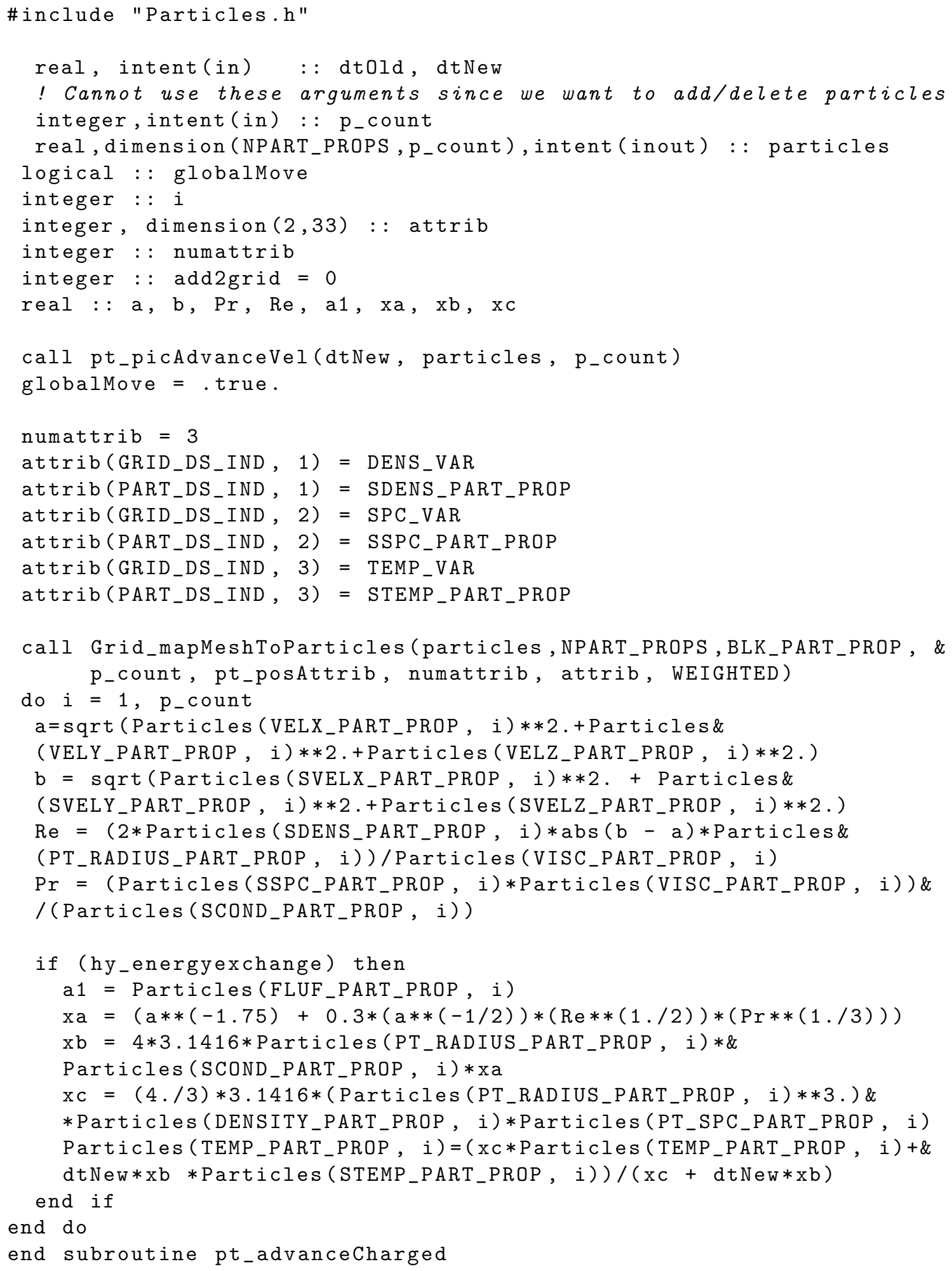




\section{A.3 Phase Change}

The phasechange model that we built in the FLASH code is located at source/Particles/ParticlesMain/active/massive/hybrid/energyexchange/phasechange. The contents of the directory are;

- Config

- Makefile

- pt_picData.F90

- pt_picInit.F90

- pt_picInitSpecies.F90

- pt_calculateVarTemp.F90

- pt_calculateTemp.F90

- pt_advanceCharged.F90

The detail subroutines are included below.

\section{A.3.1 Config}

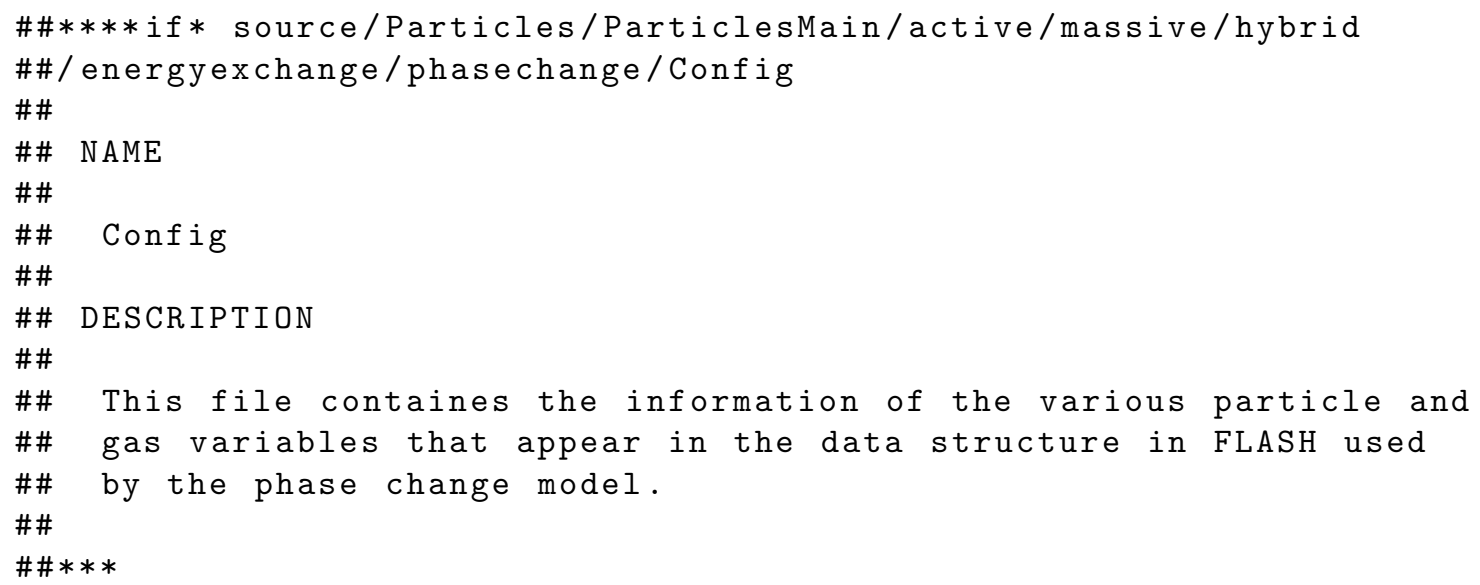




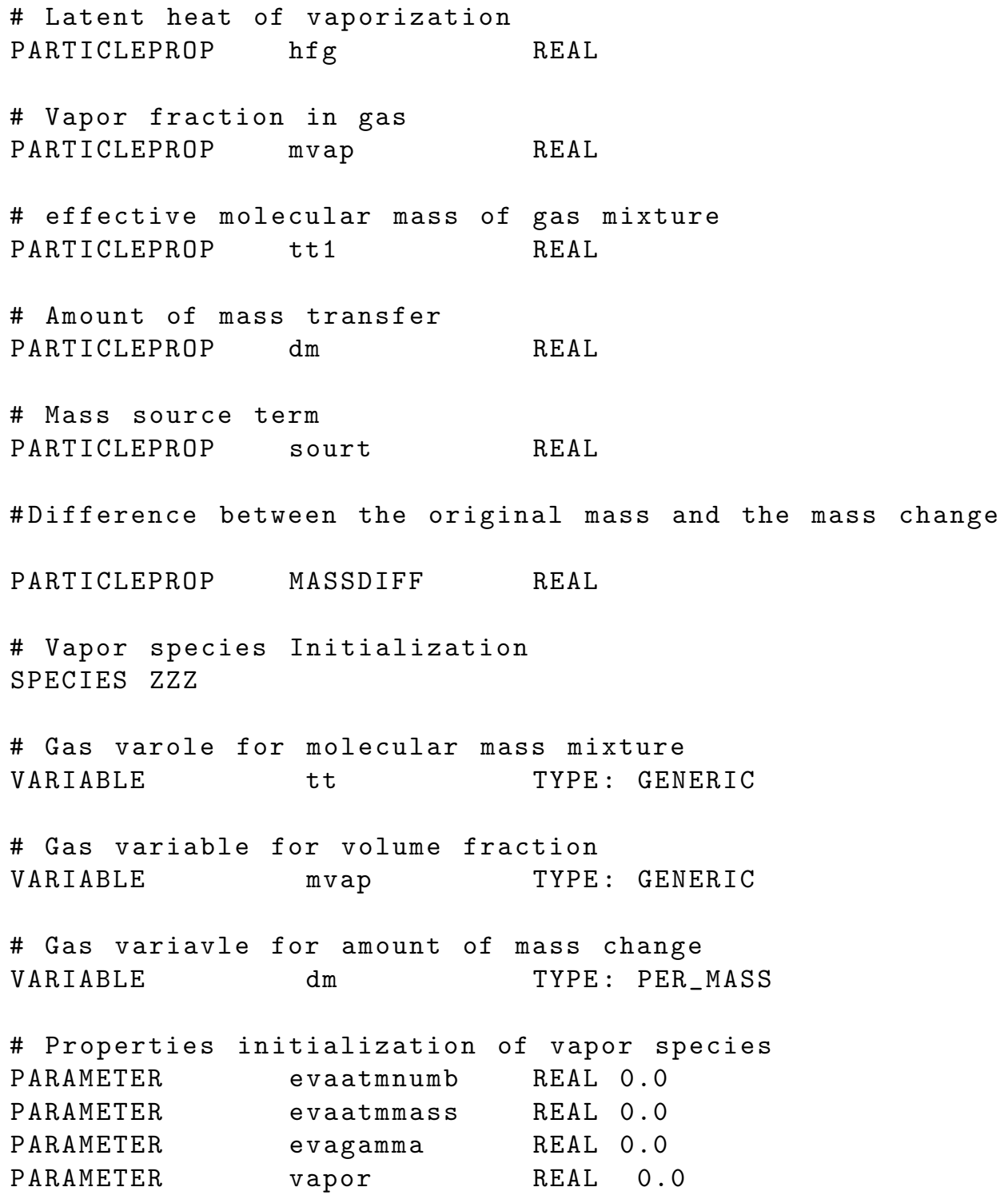

\section{A.3.2 pt_picData}

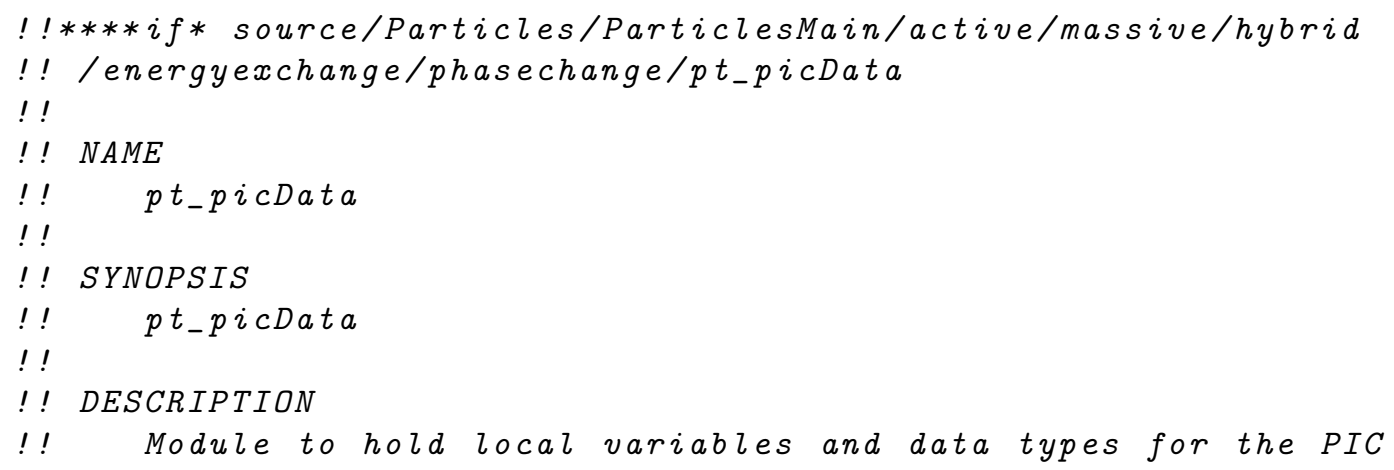




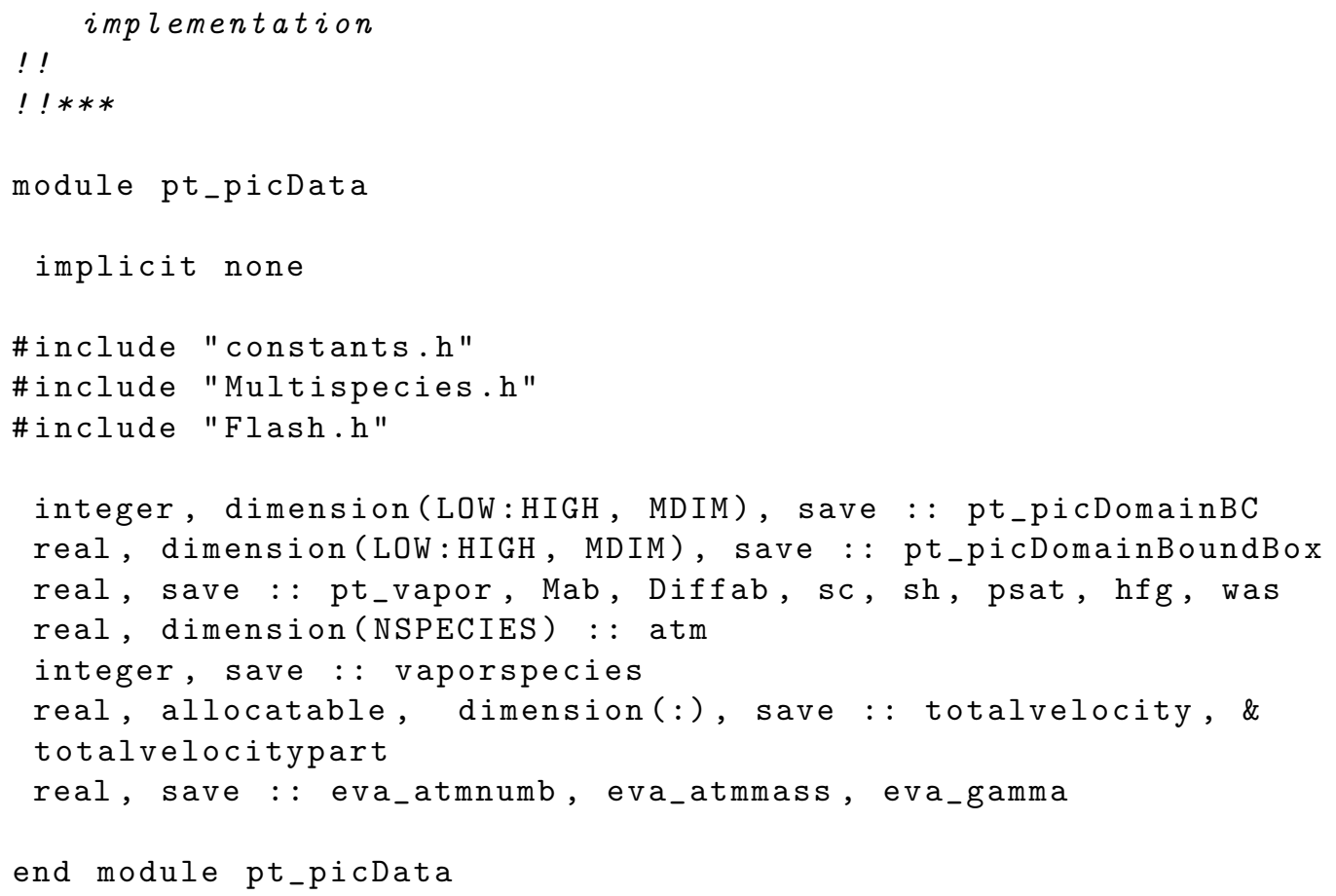

\section{A.3.3 pt_picInit}

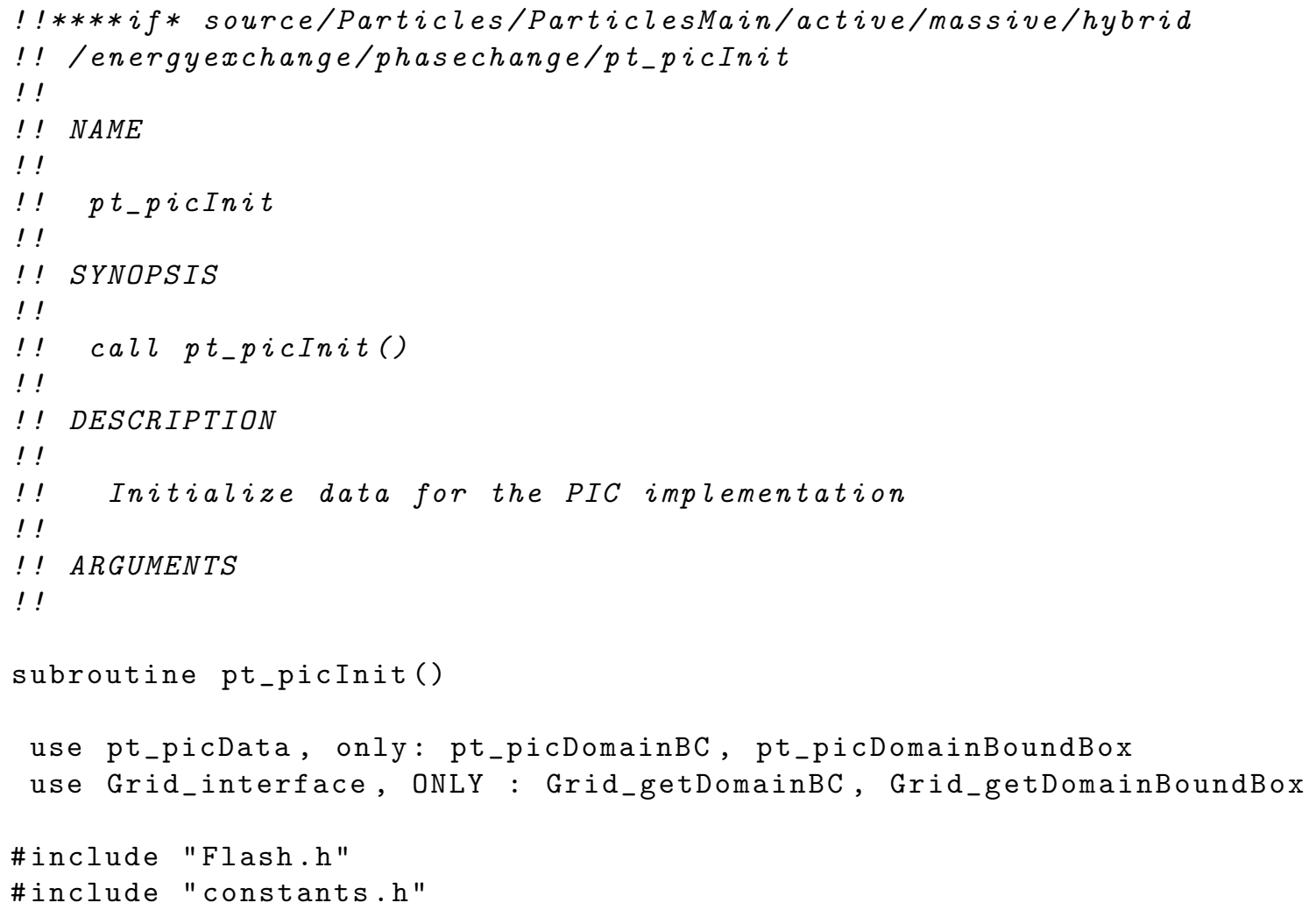




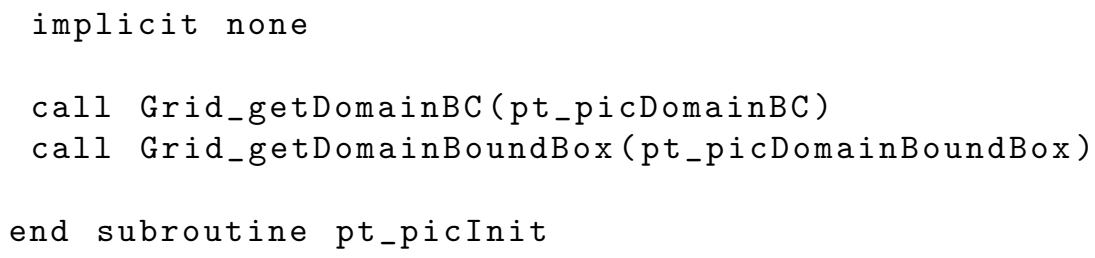

\section{A.3.4 pt_initSpecies}

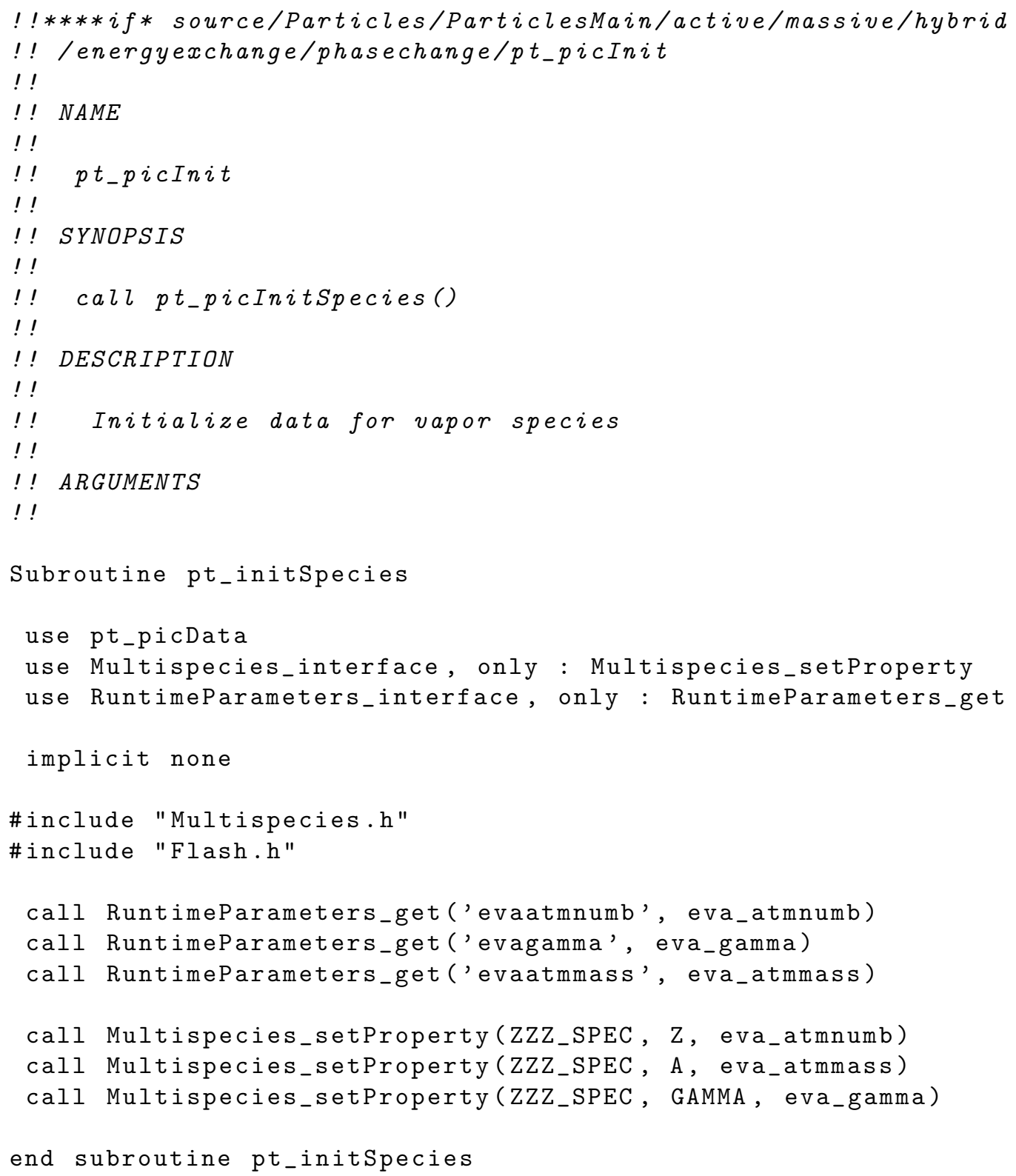

\section{A.3.5 pt_calculateVarTemp}




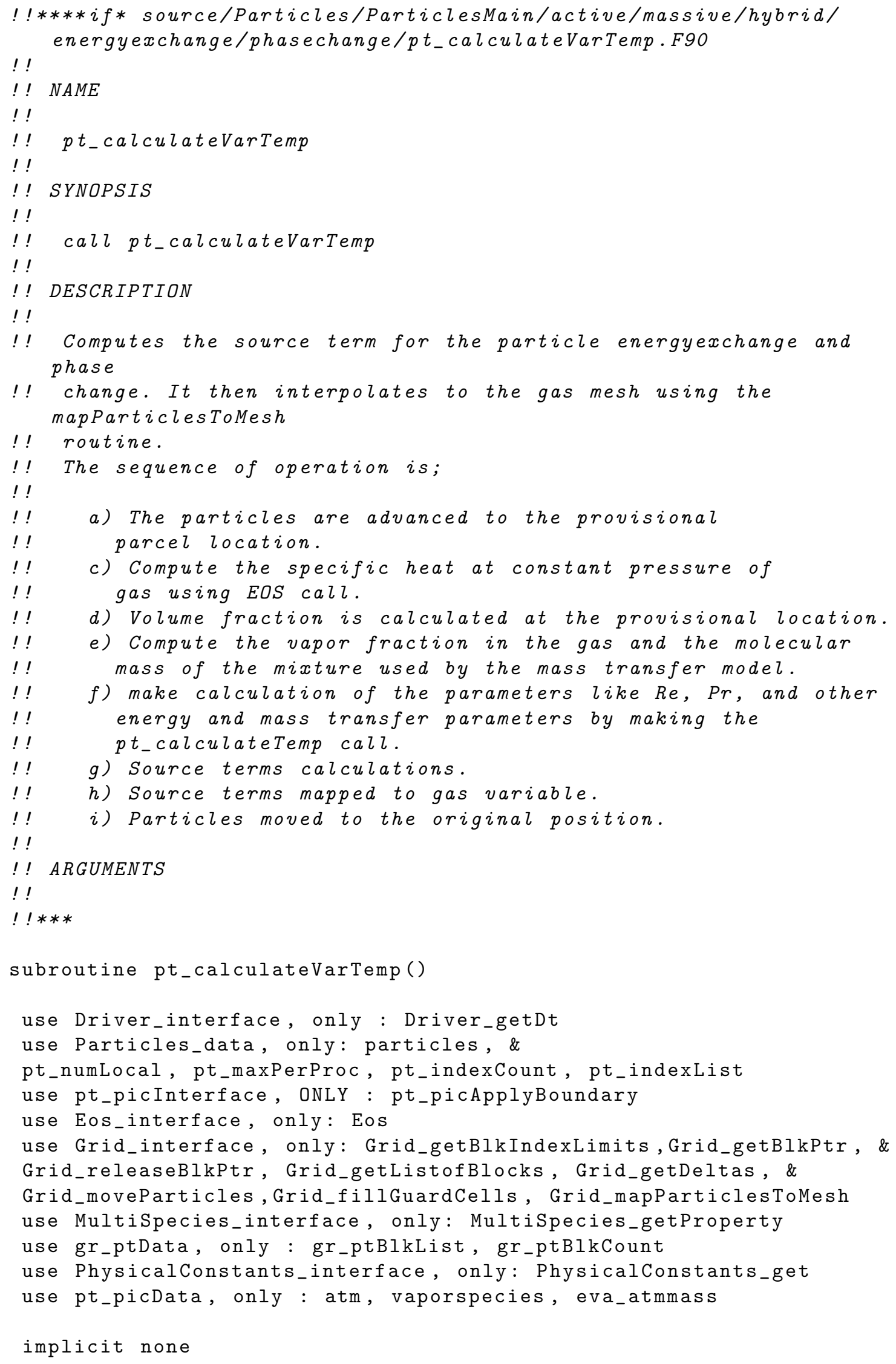




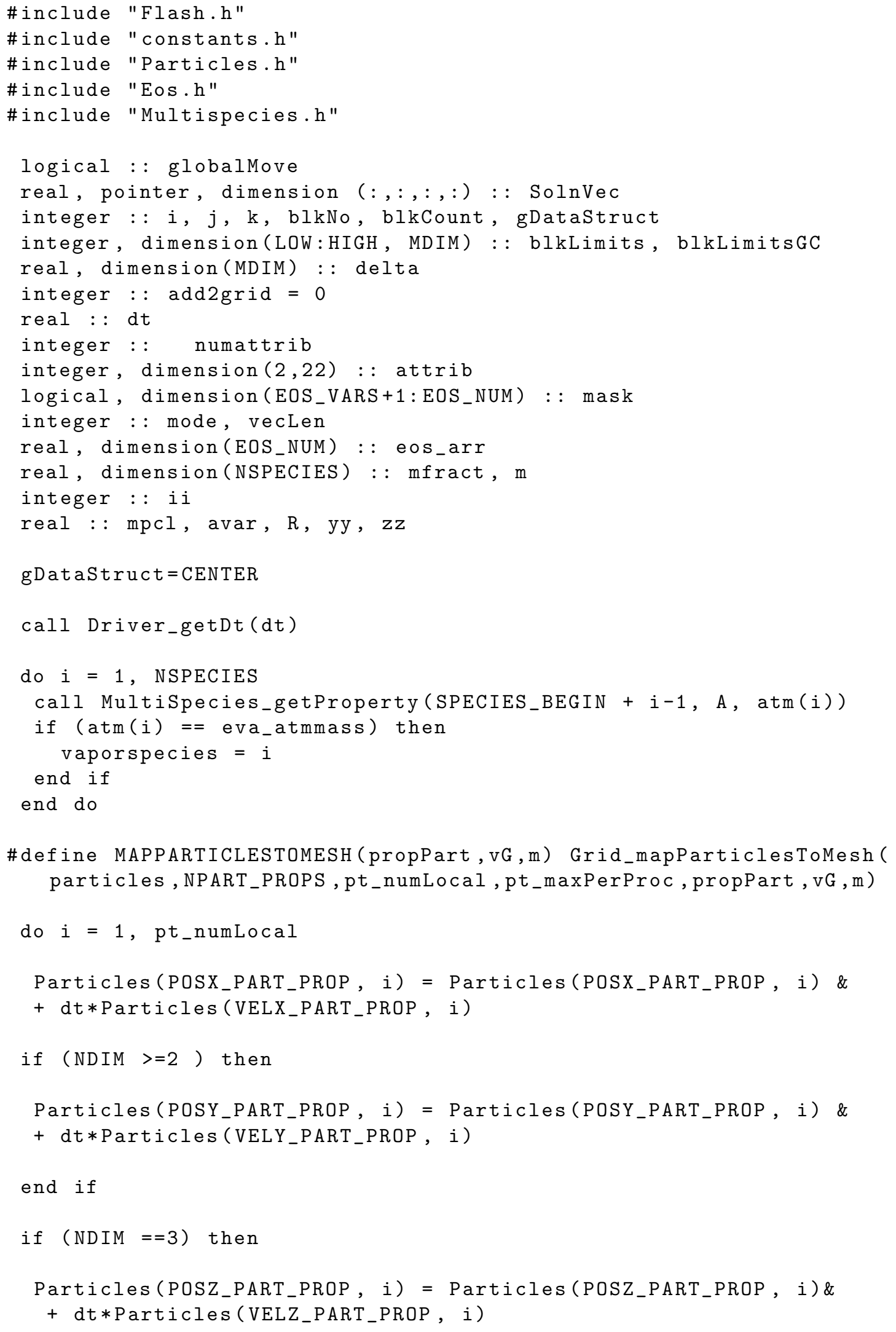




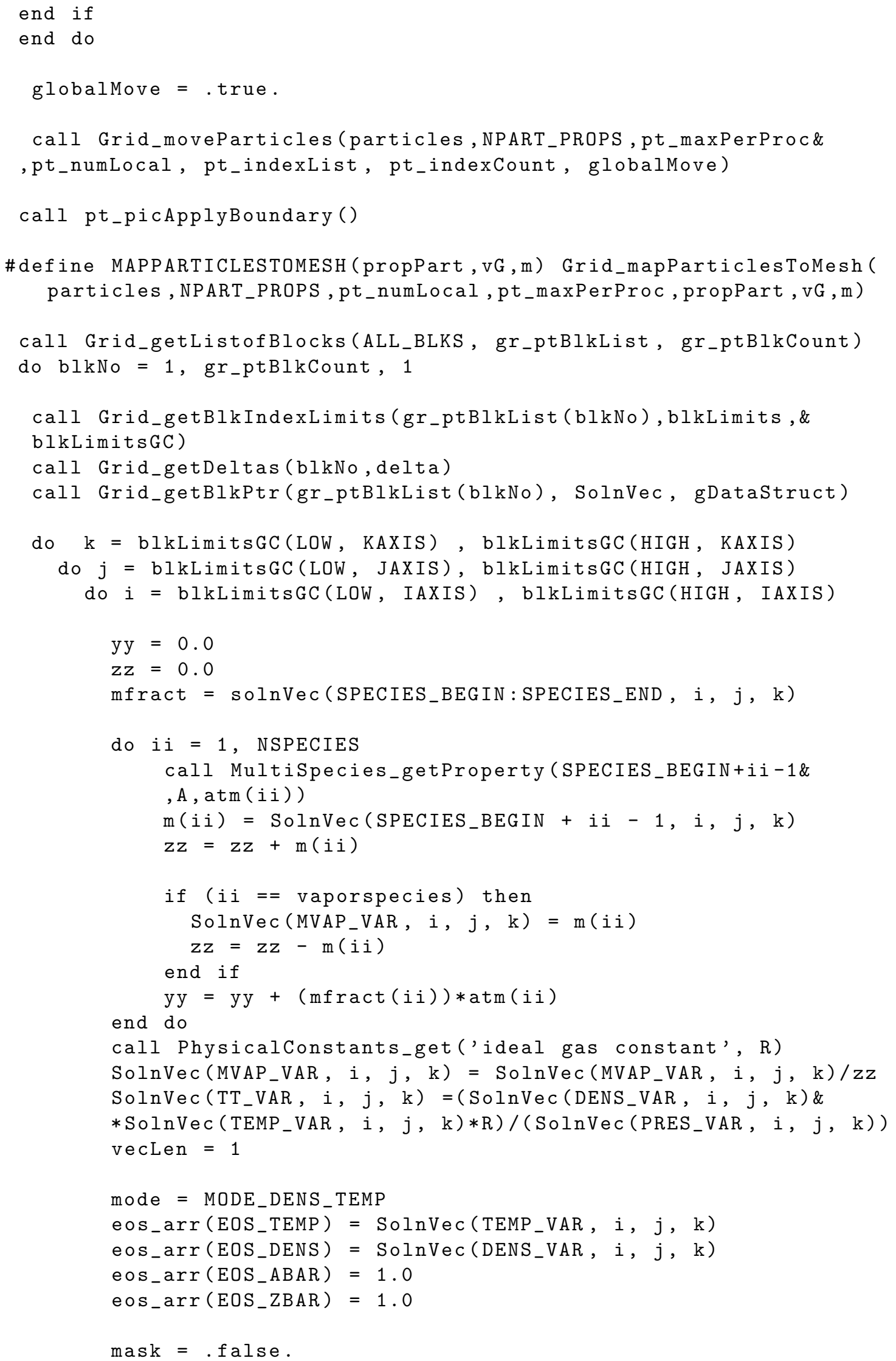




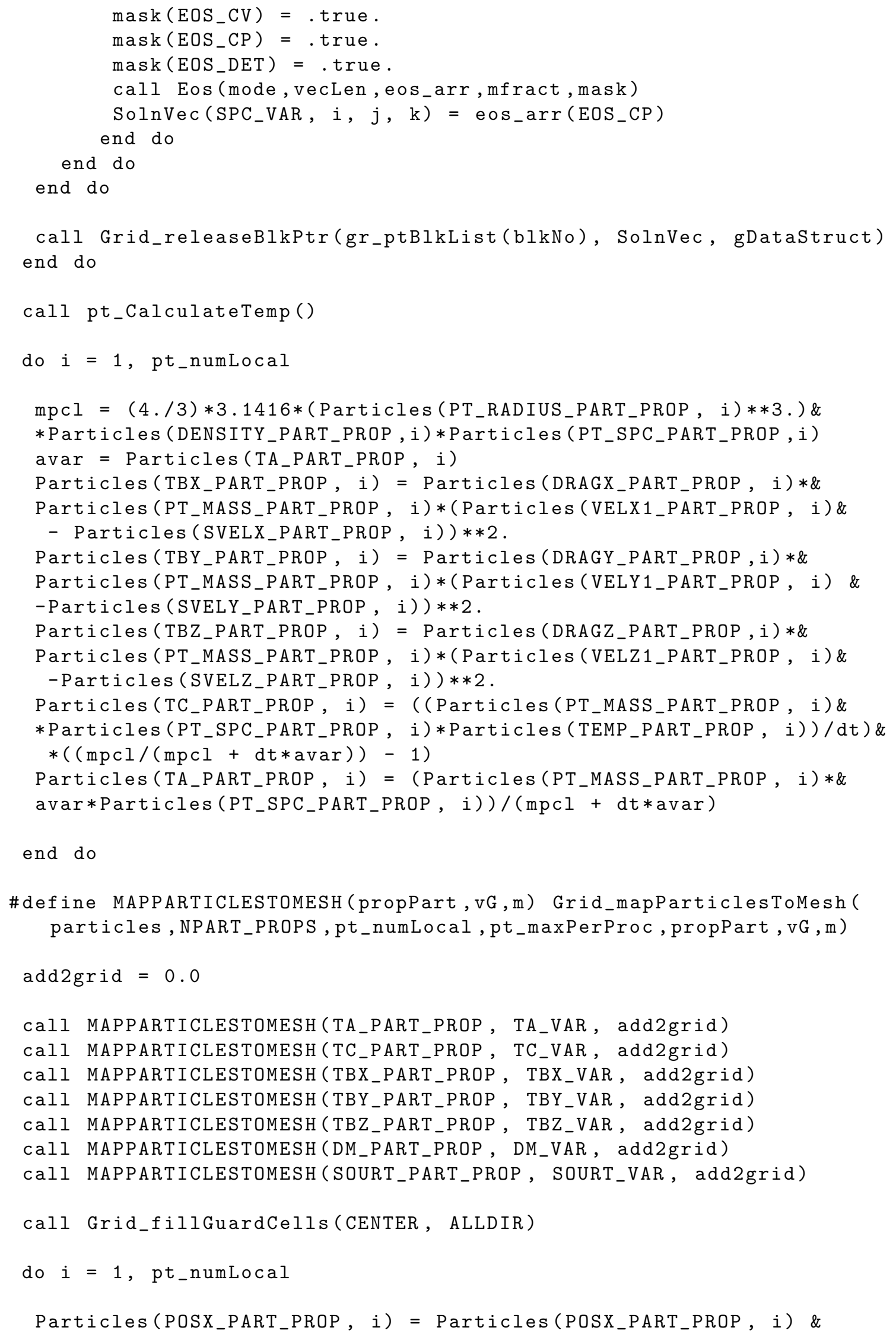




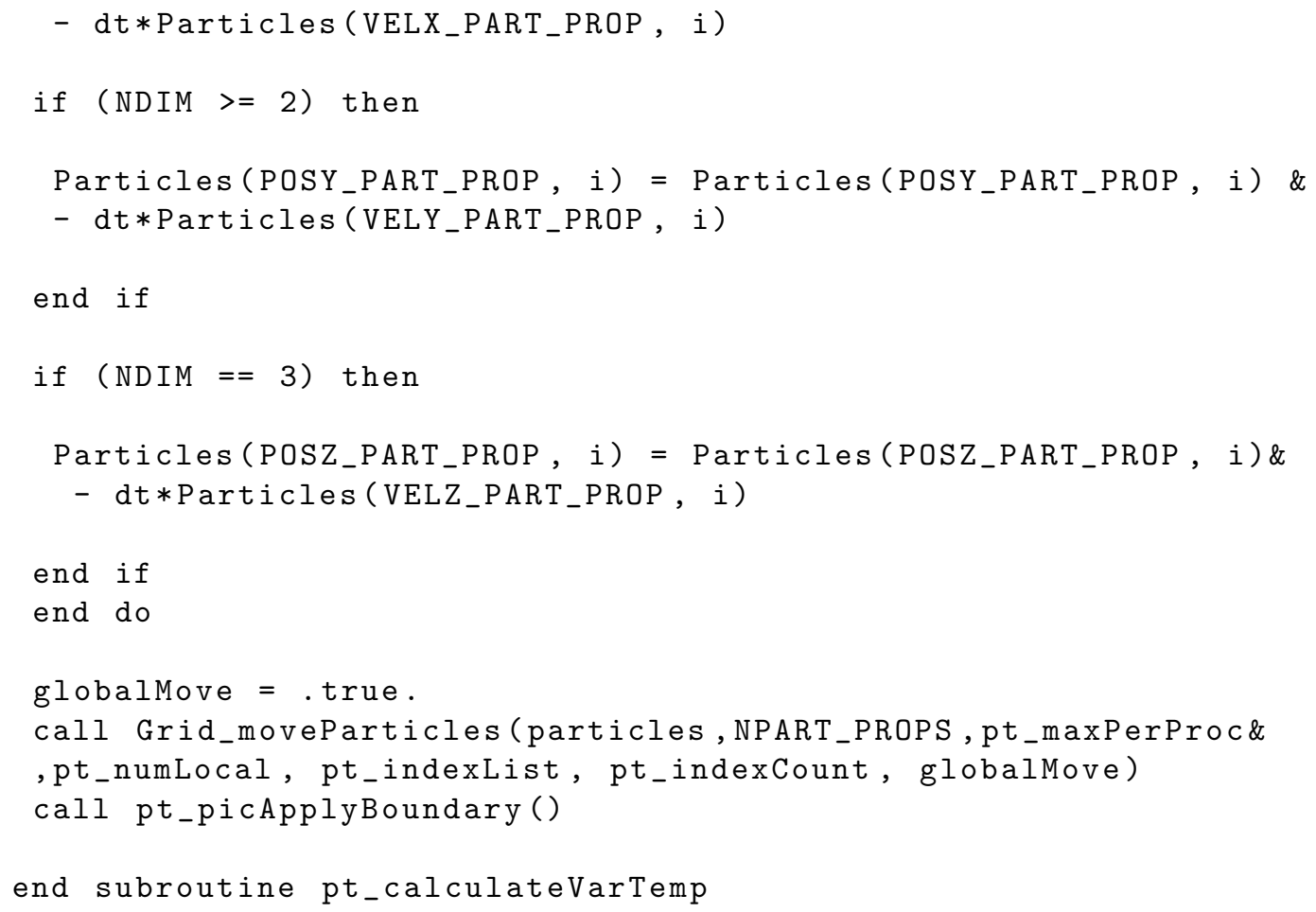

\section{A.3.6 pt_calculateTemp}

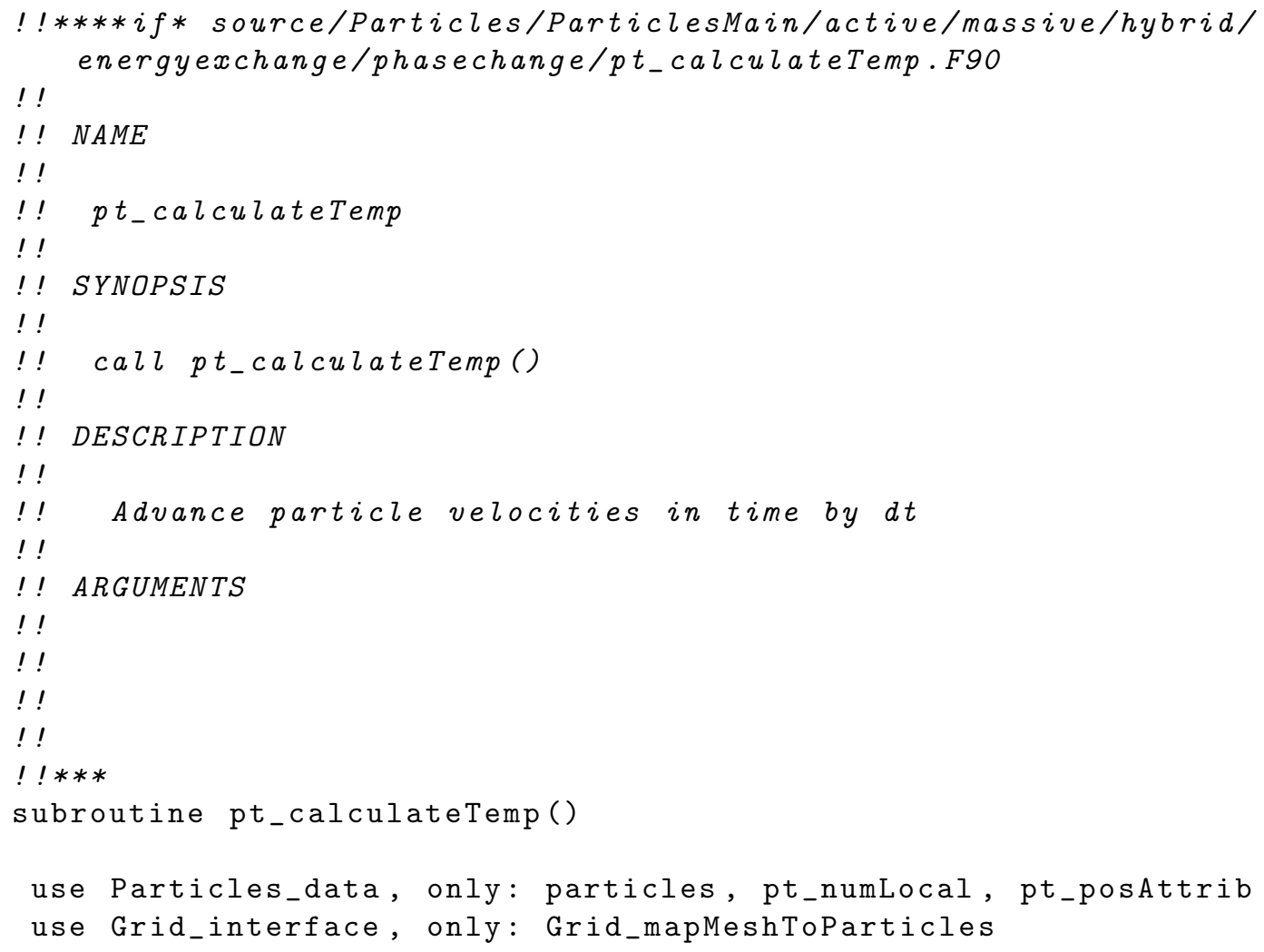




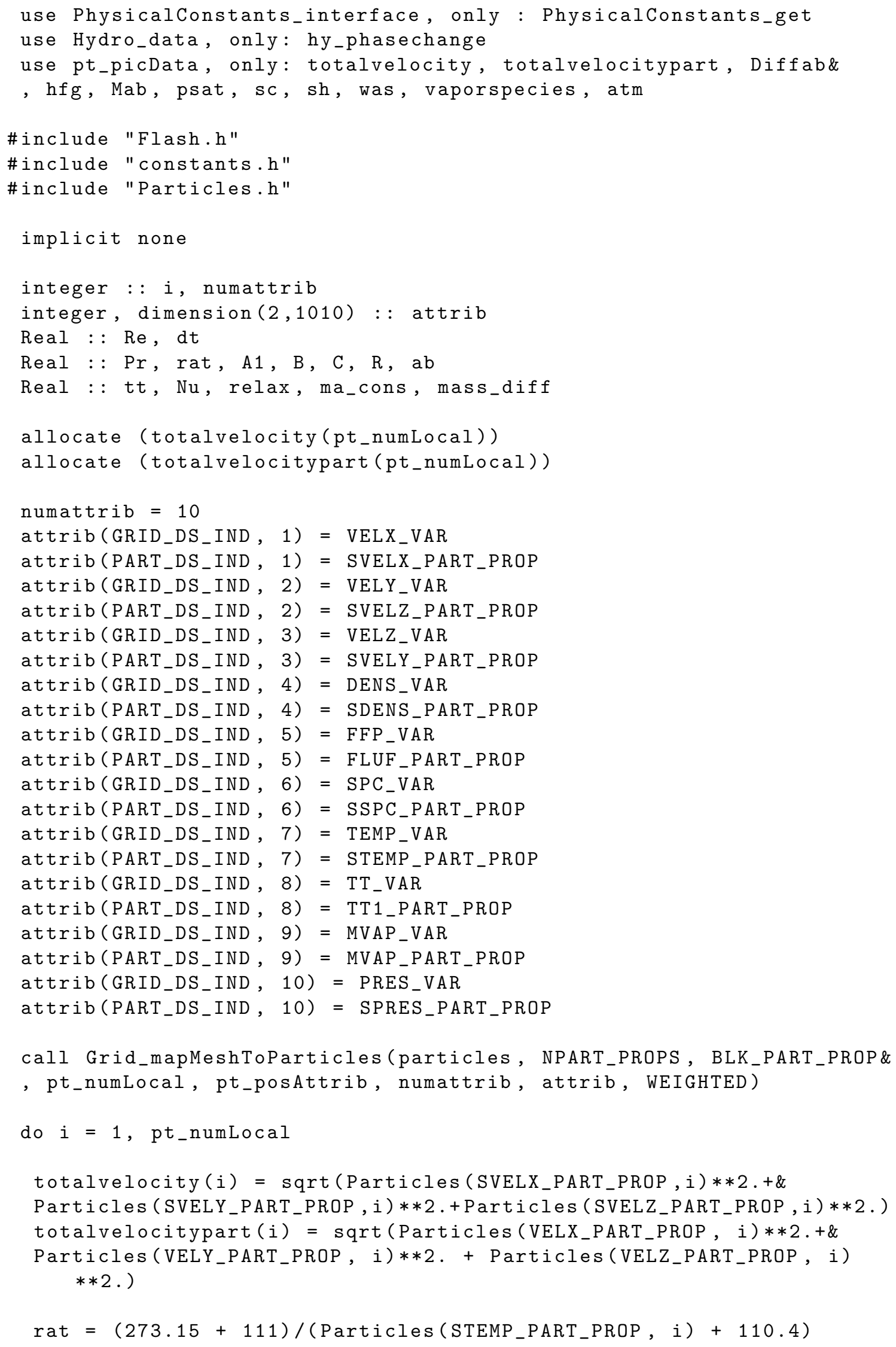




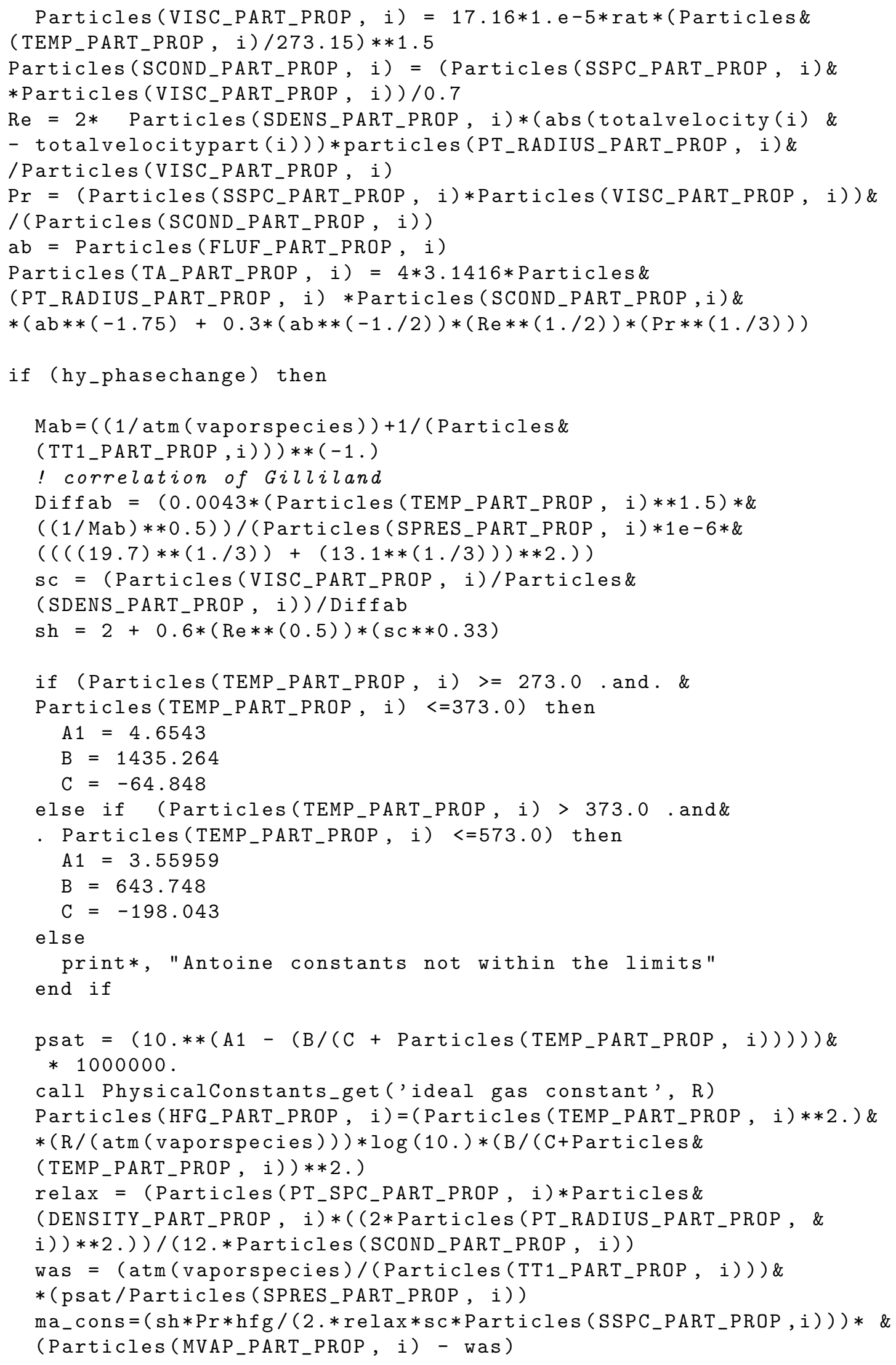




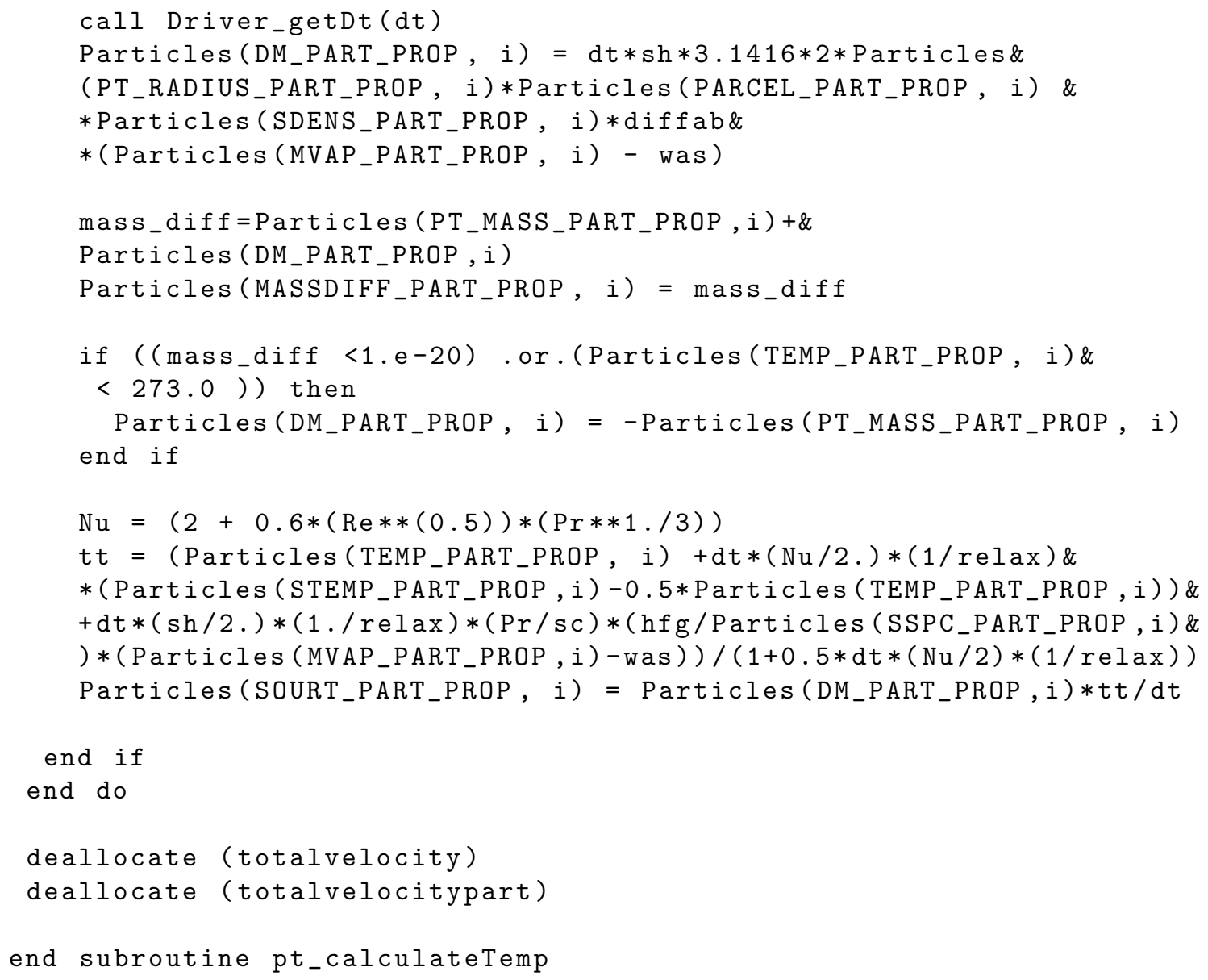

\section{A.3.7 pt_advanceCharged}

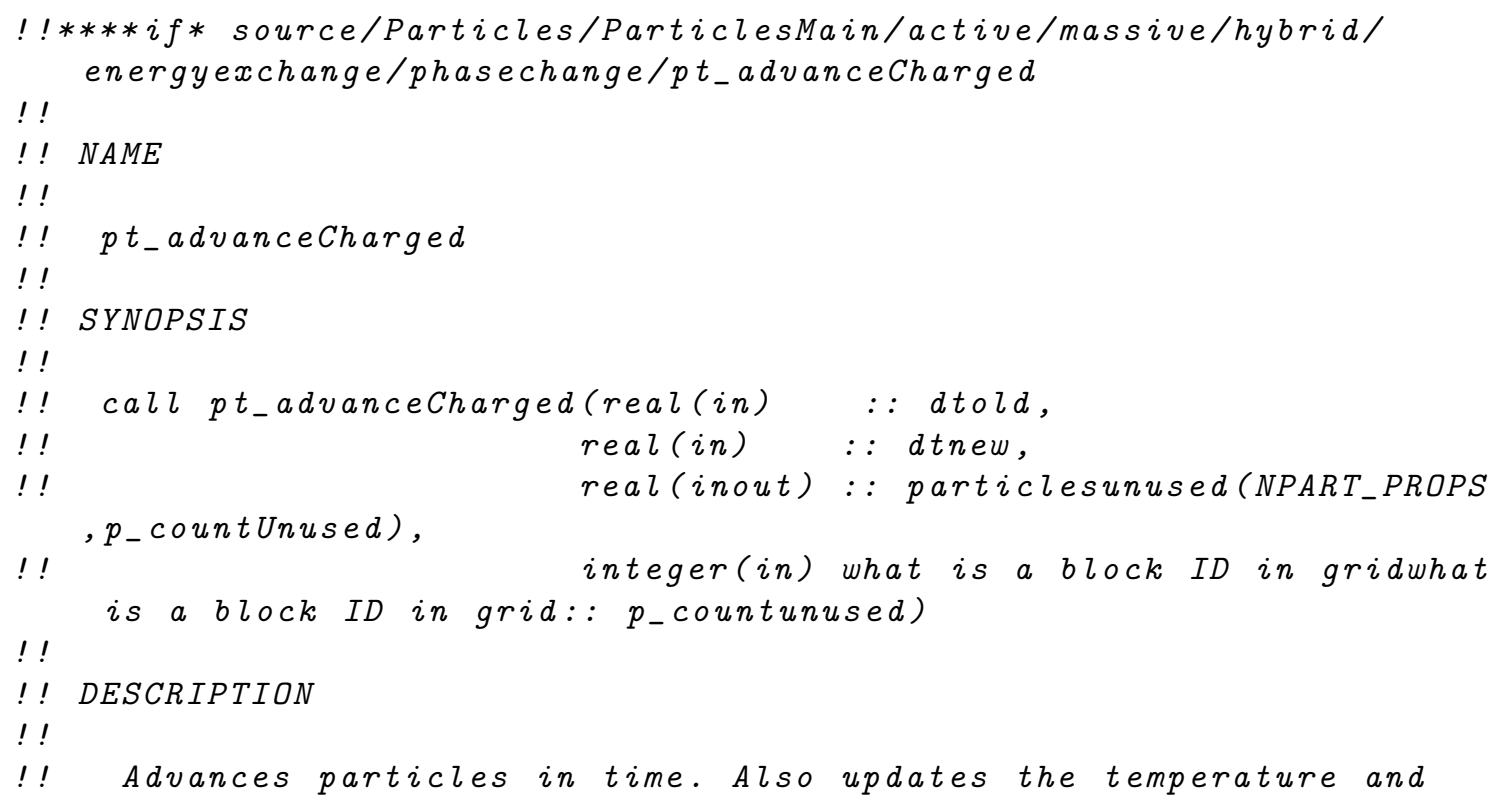




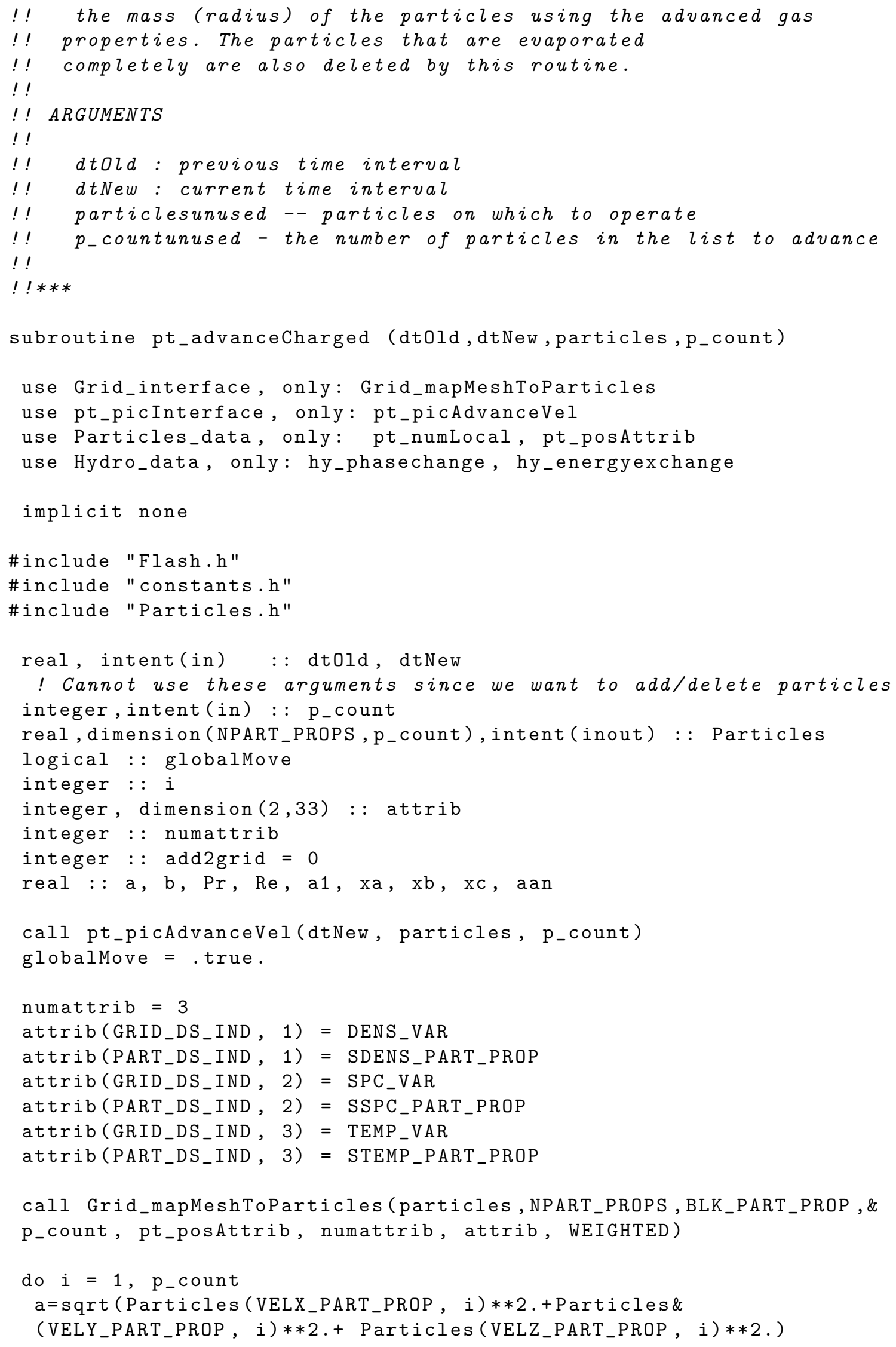




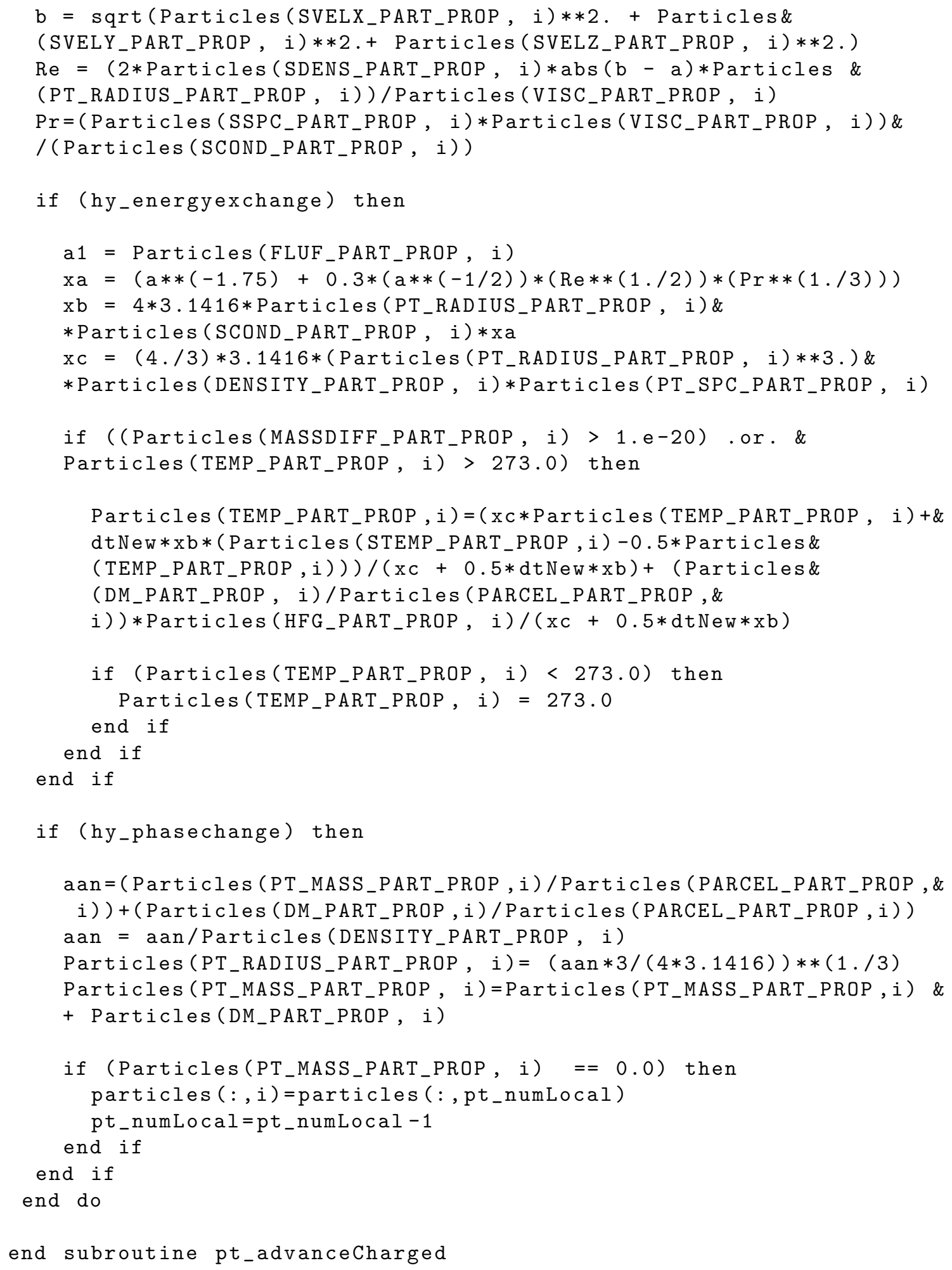




\section{A.4 Hydro}

The conservation equations of the gas are solved by using the hydro model in PPM. To include the particle effects, the hydro has to be modified with the particle source terms. A new directory was added at source/physics/Hydro/HydroMain/split/PPM named the active_part. The directory contains the modified hydro files. The Config file of the hydro was modified so that the +mom setup argument while compiling FLASH would copy the files from active_part to the object directory. The contents of the active_ part directory are;

- Hydro.F90

- hy_ppm_sweep.F90

- hy_ppm_updateMom.F90

- Makefile

\section{A.4.1 Hydro.F90}

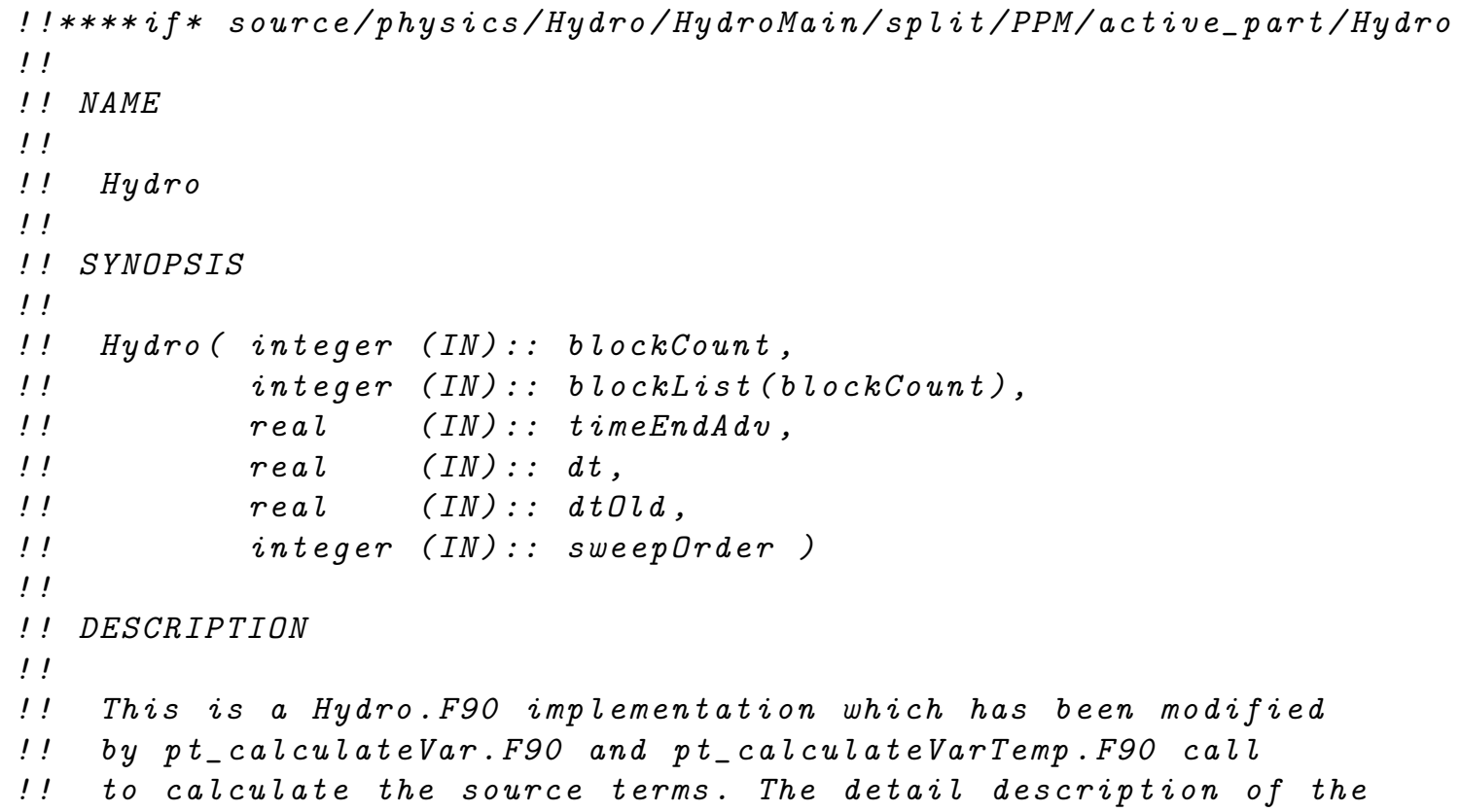




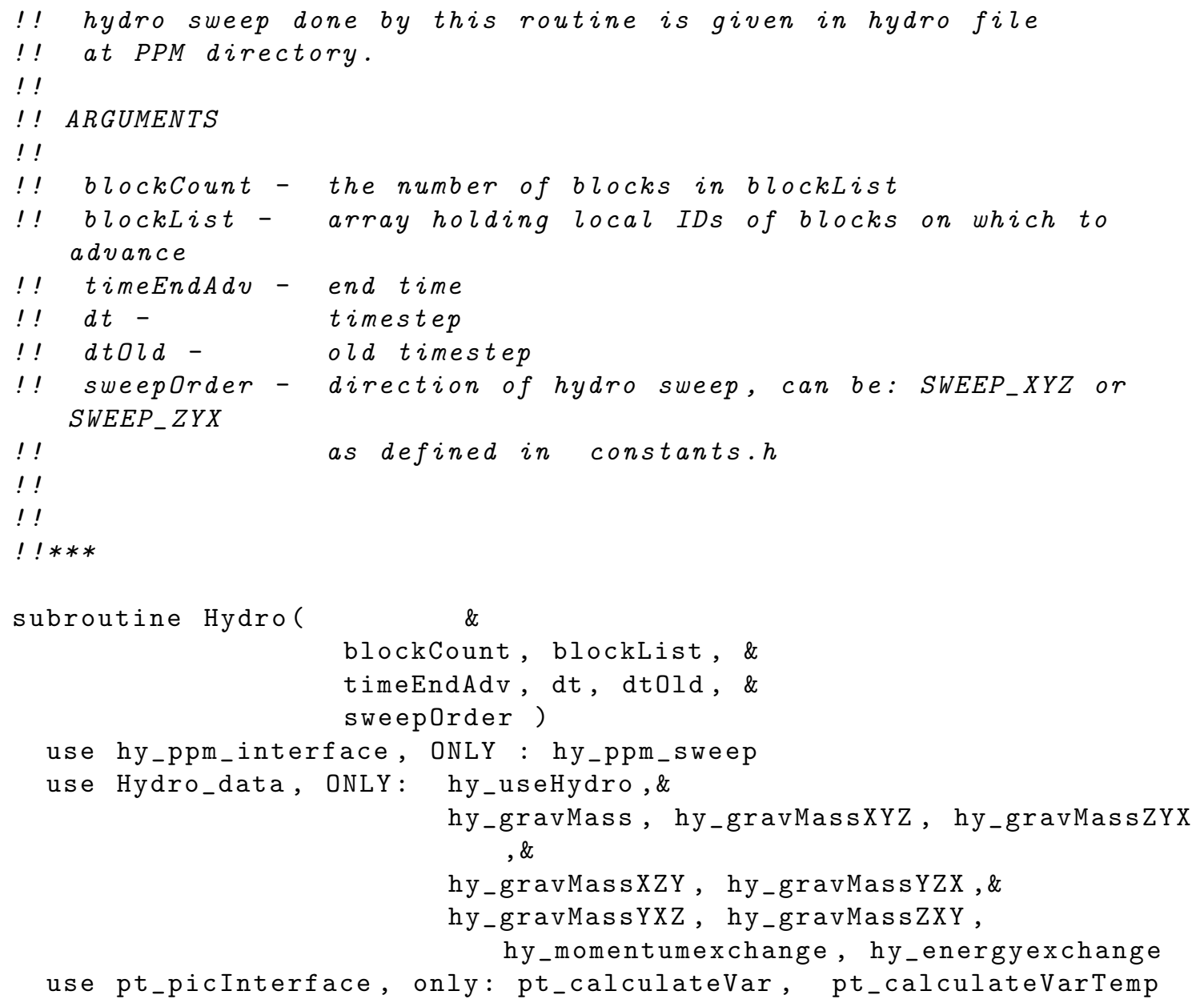




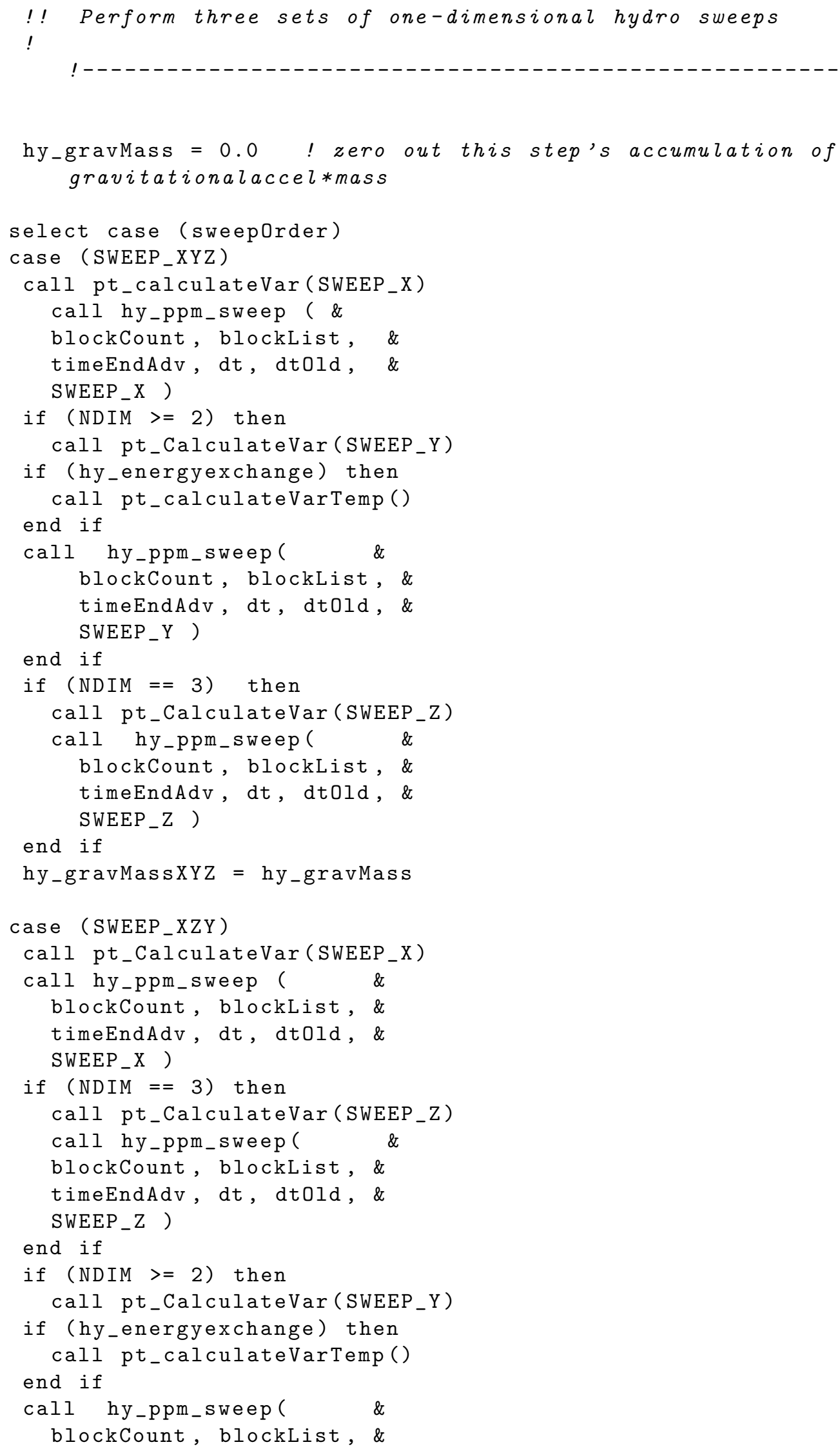




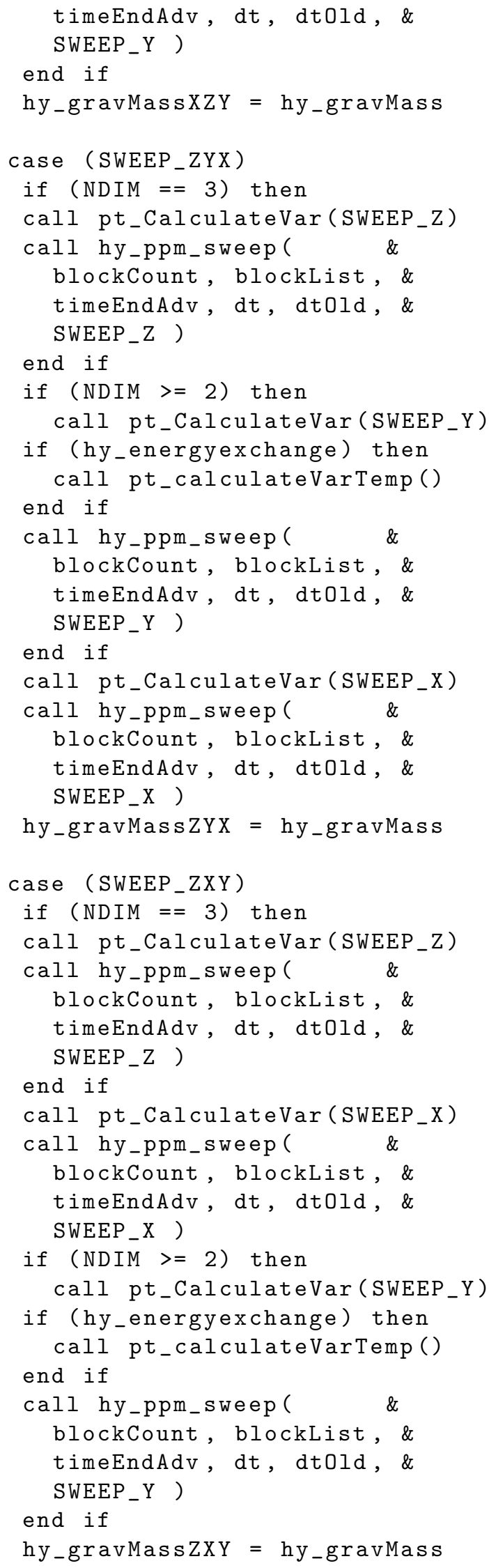




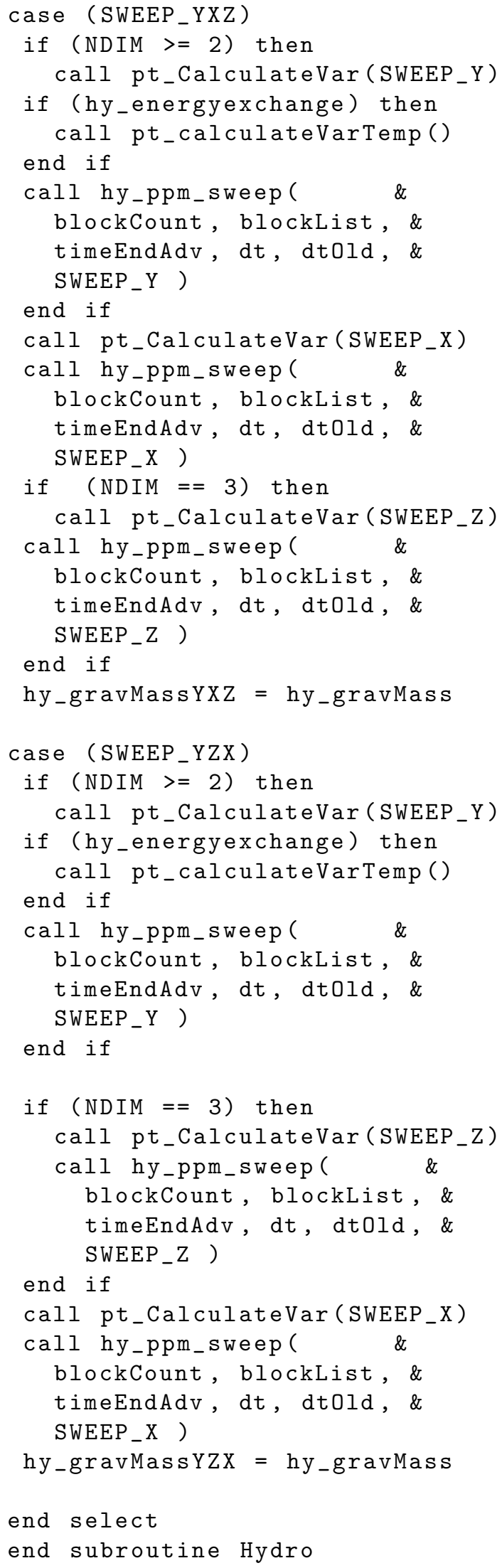




\section{A.4.2 hy_ppm_updateMom.F90}

The routine in FLASH that solves the PPM conservation equation is hy_ppm_updateSoln.F90. We have modified that subroutine with the particle source terms and renamed as hy_ppm_updateMom.F90. The details of the routine is given below.

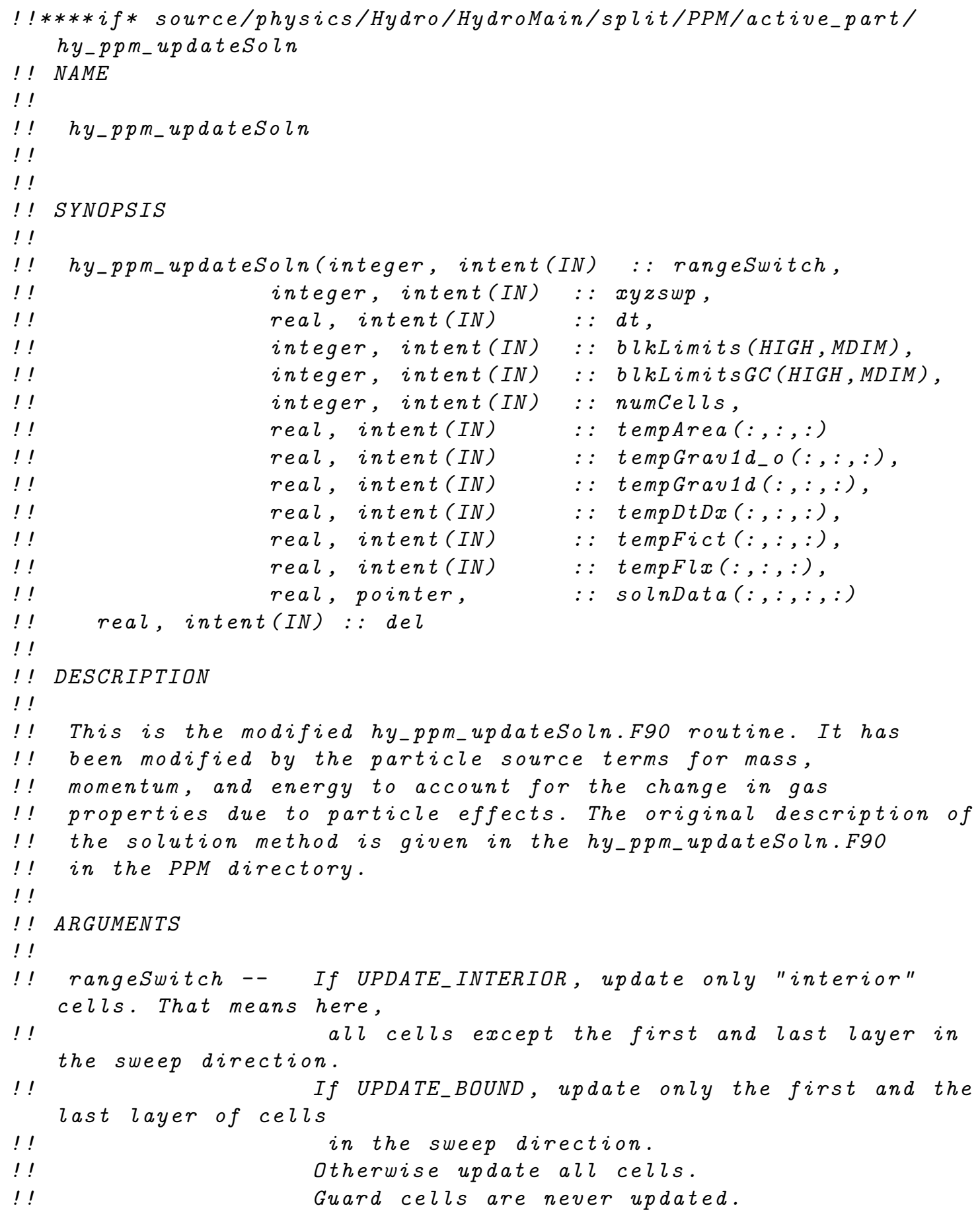




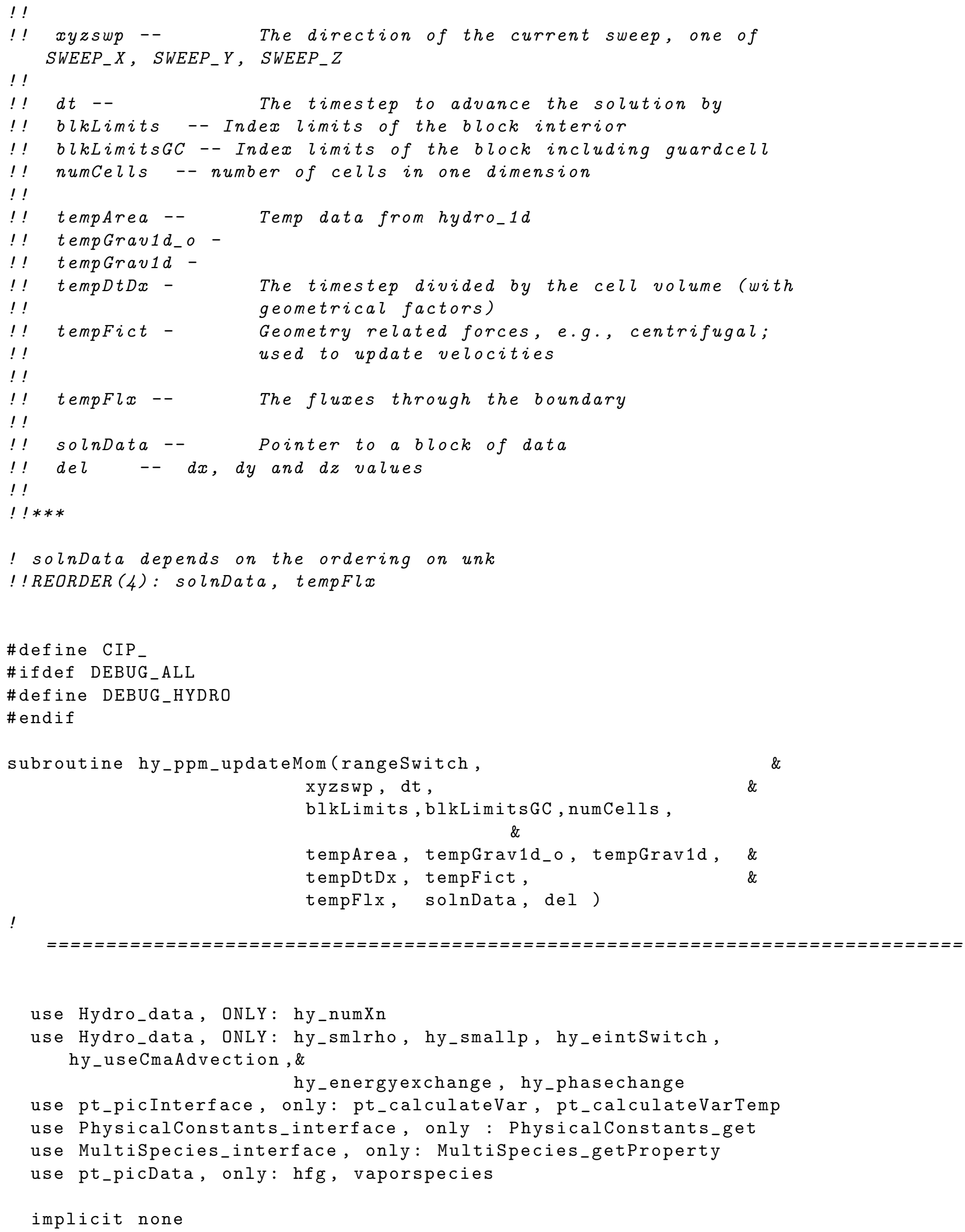




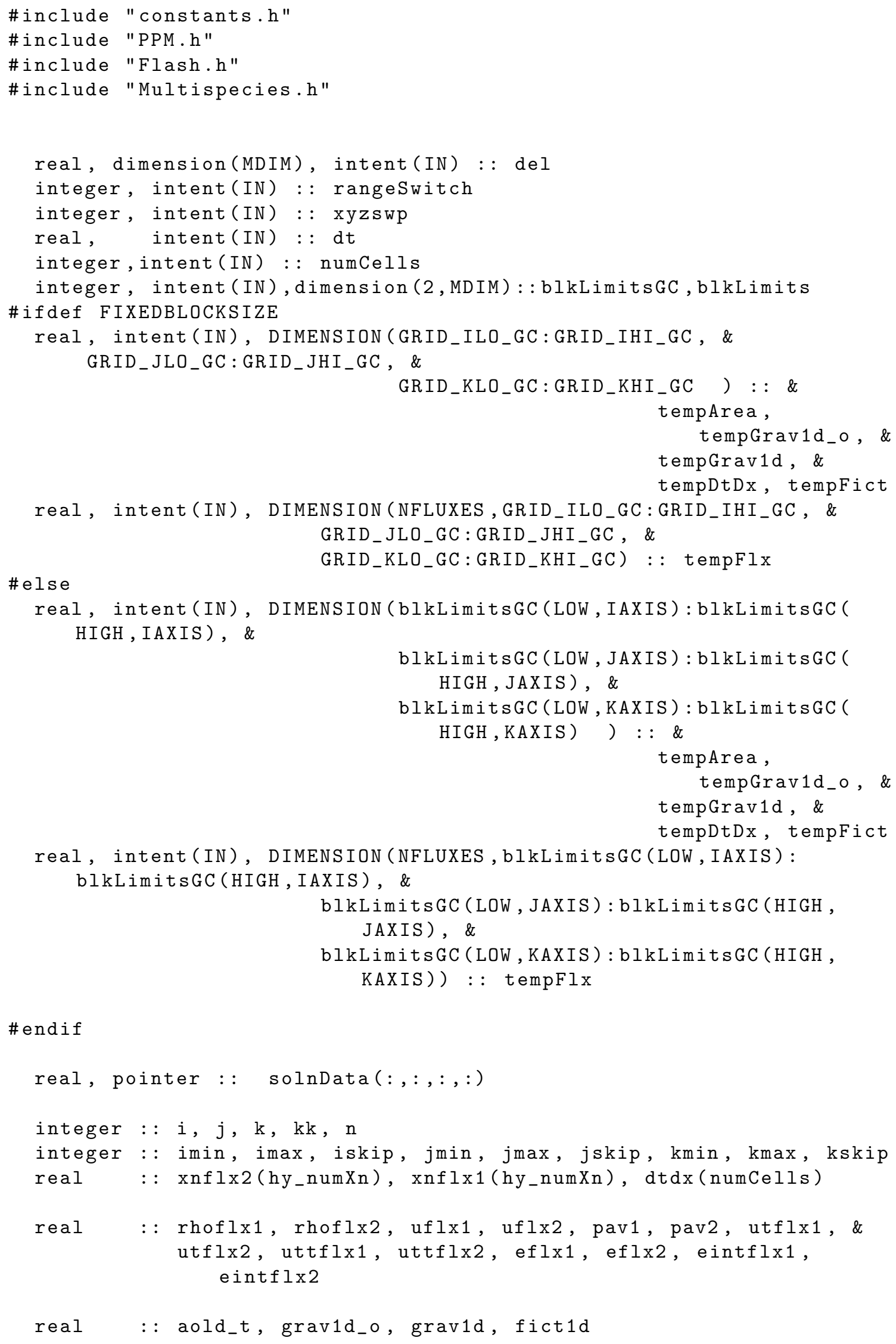




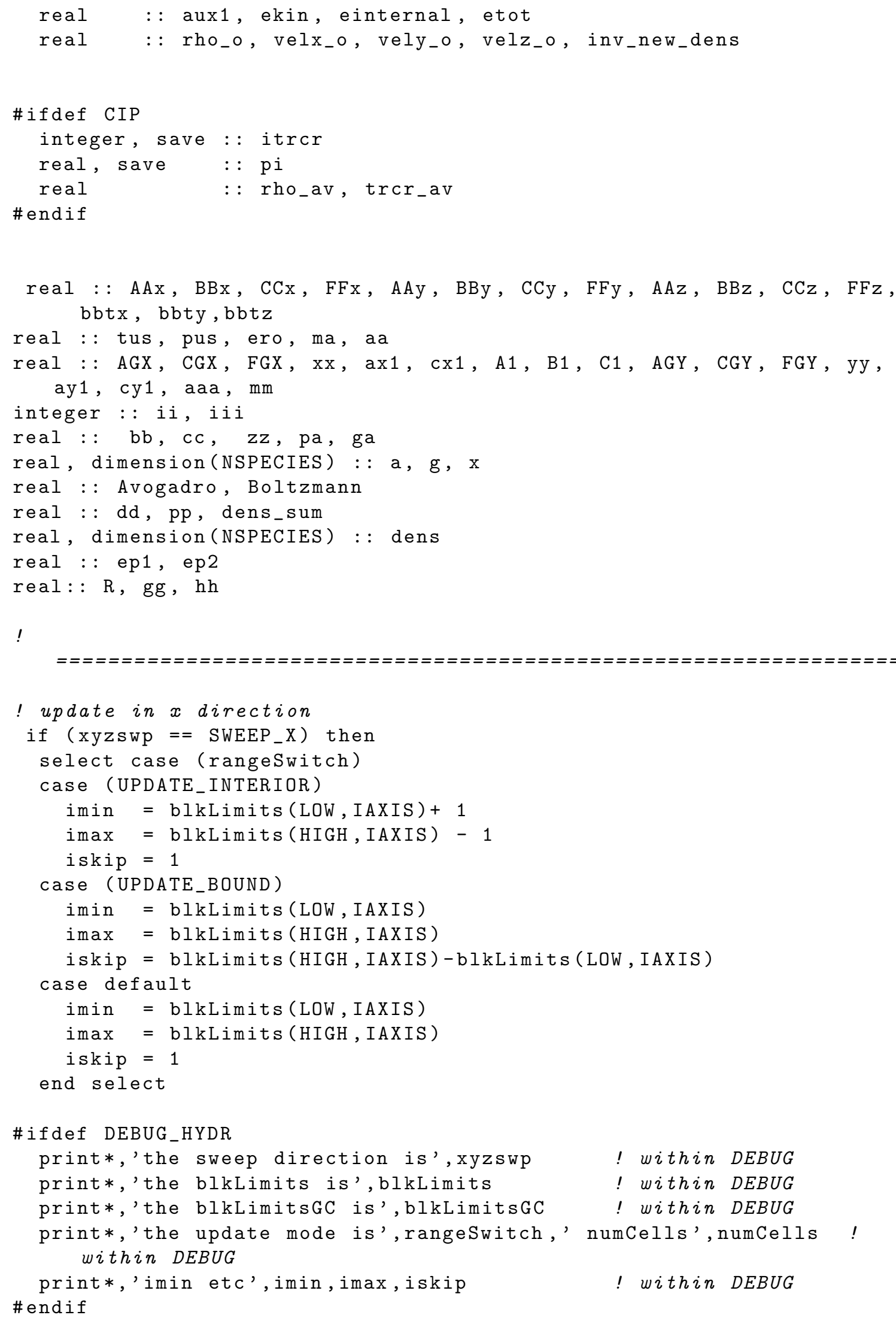




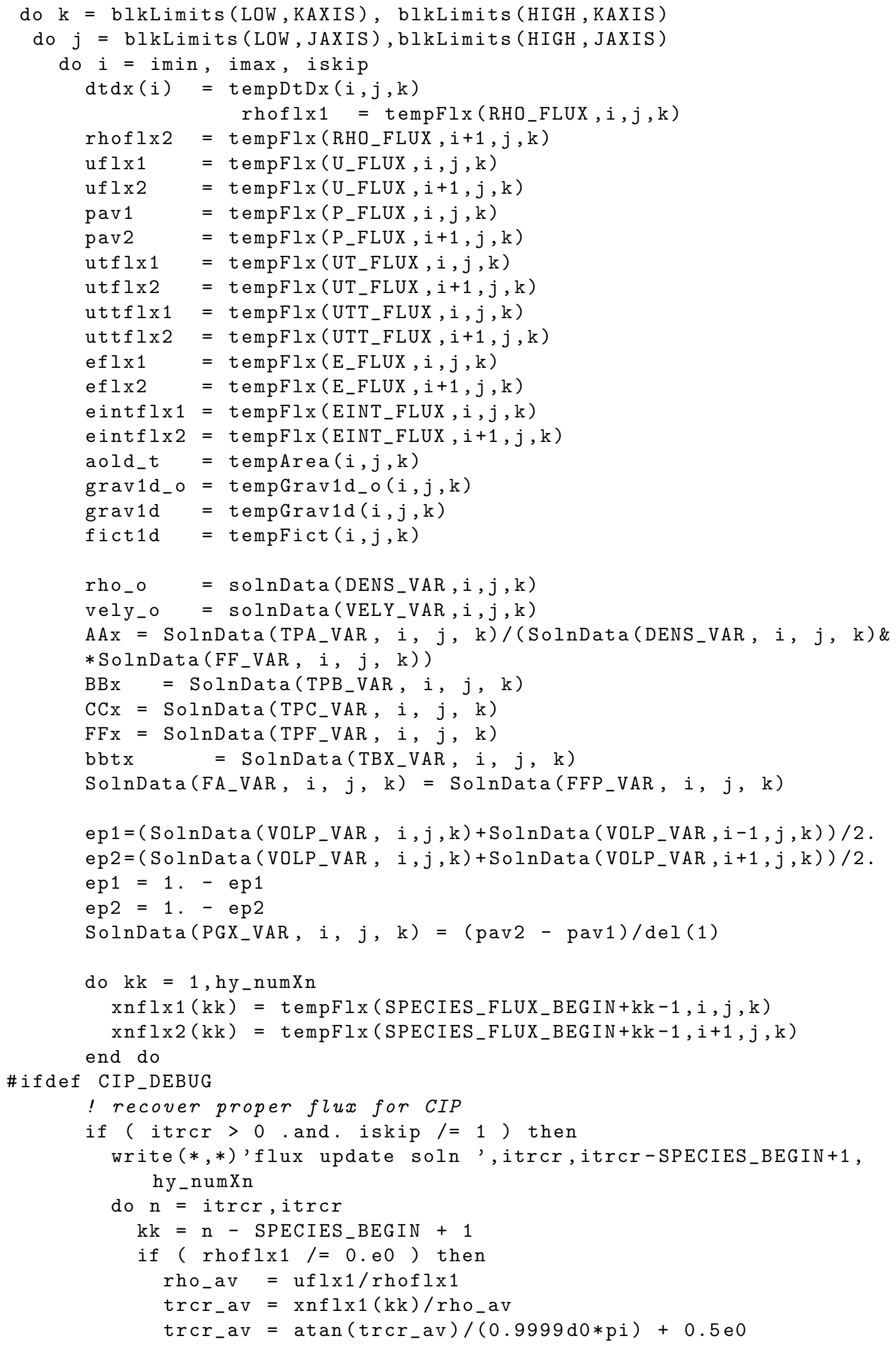




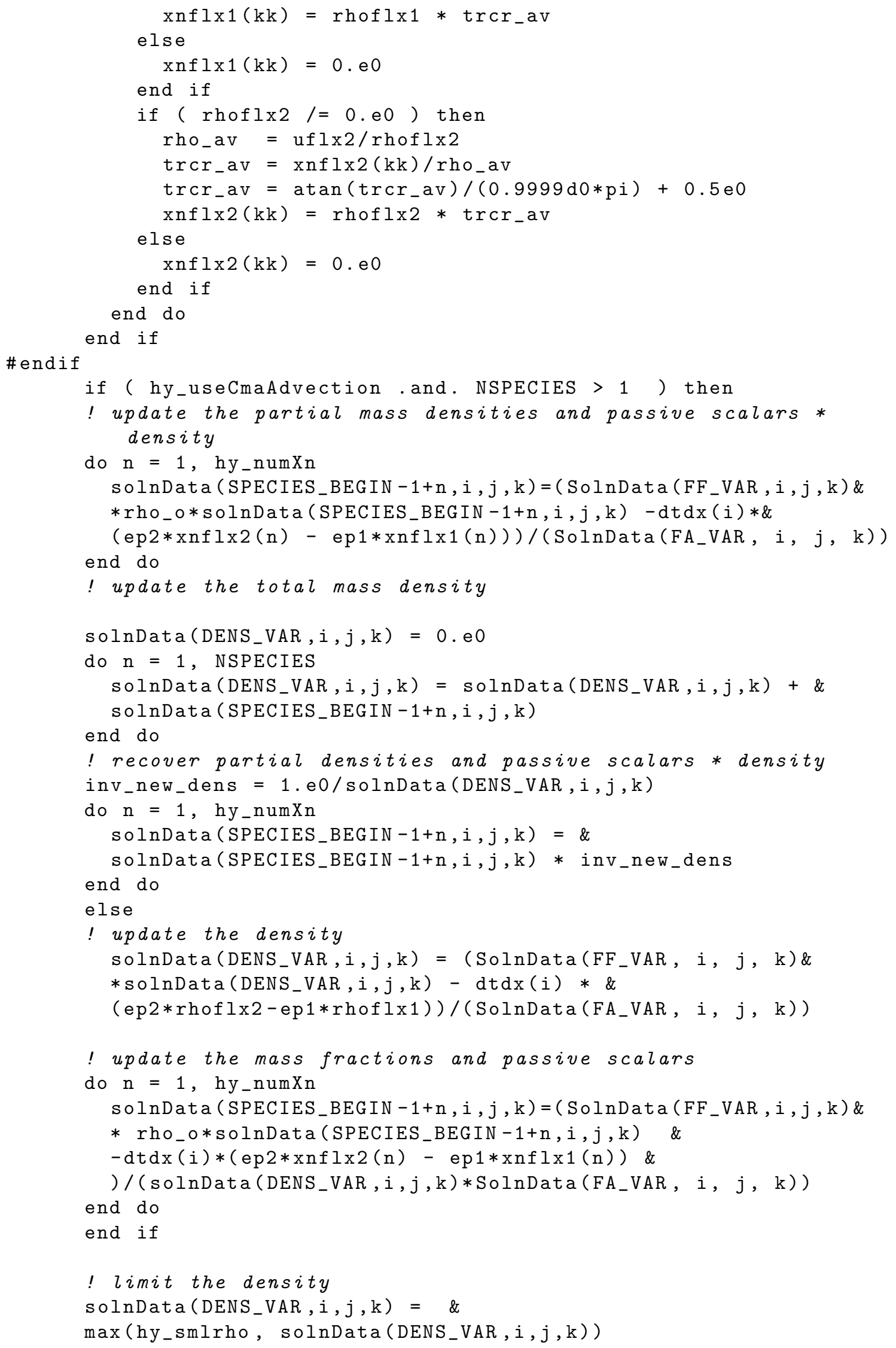




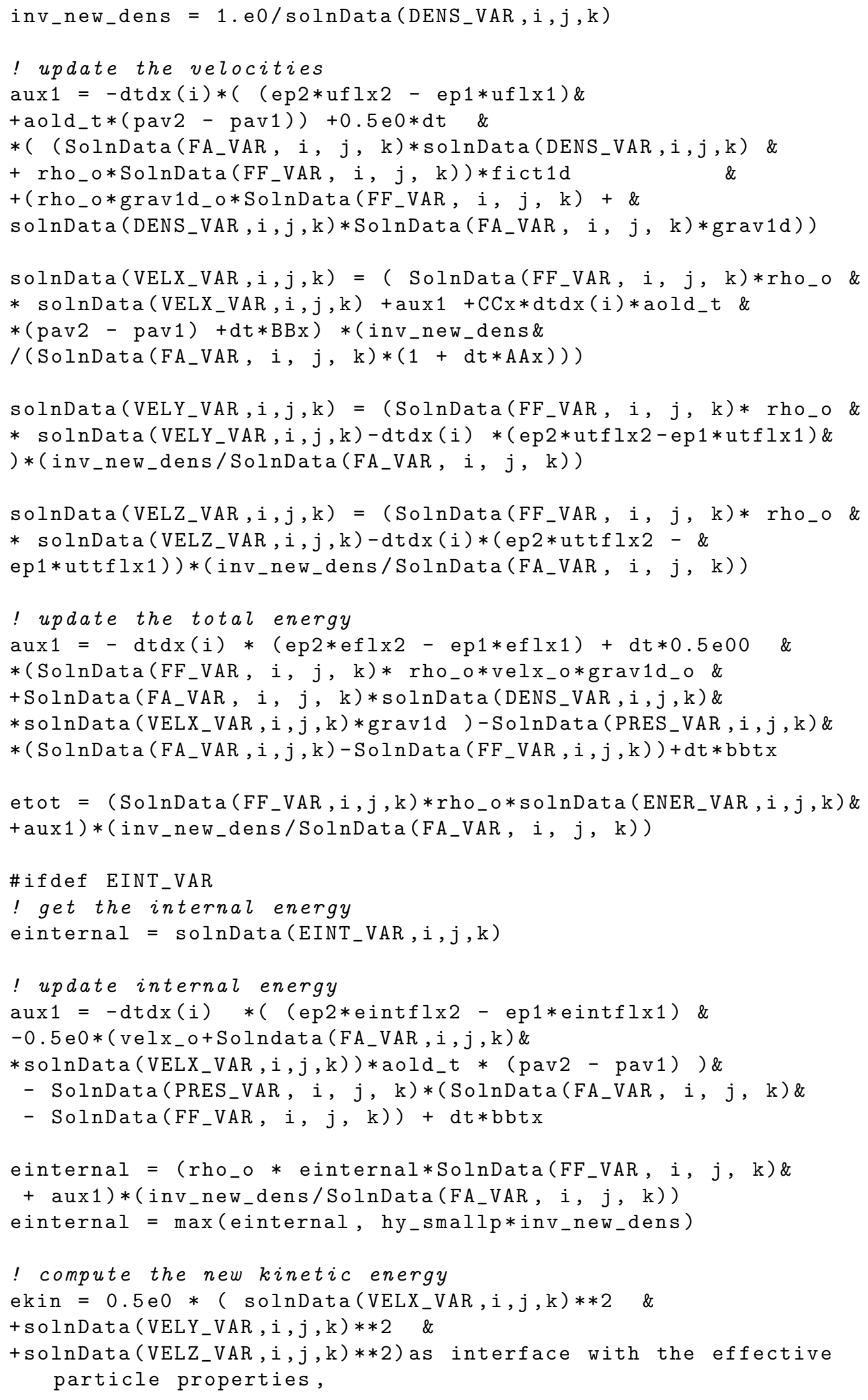




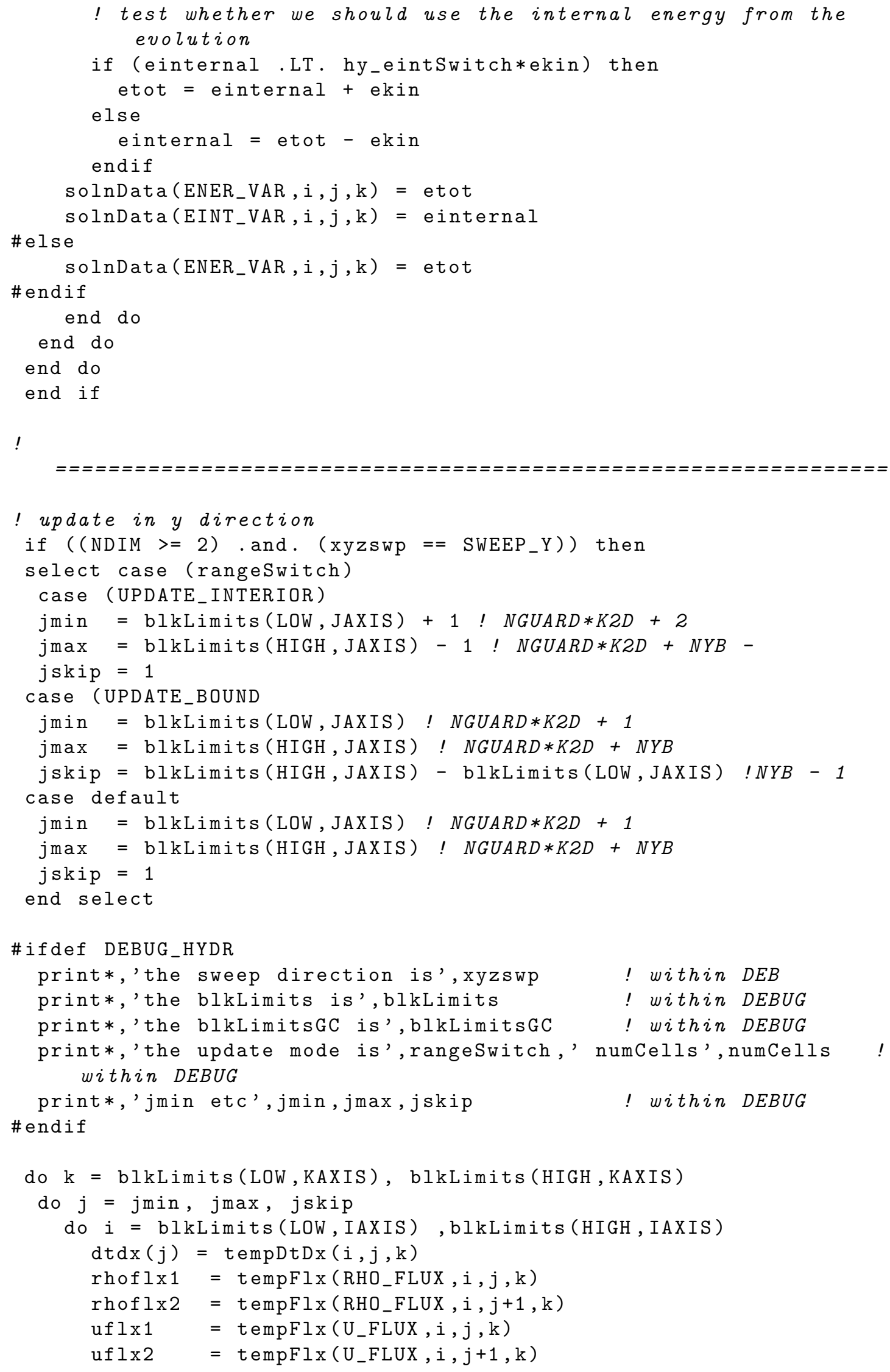




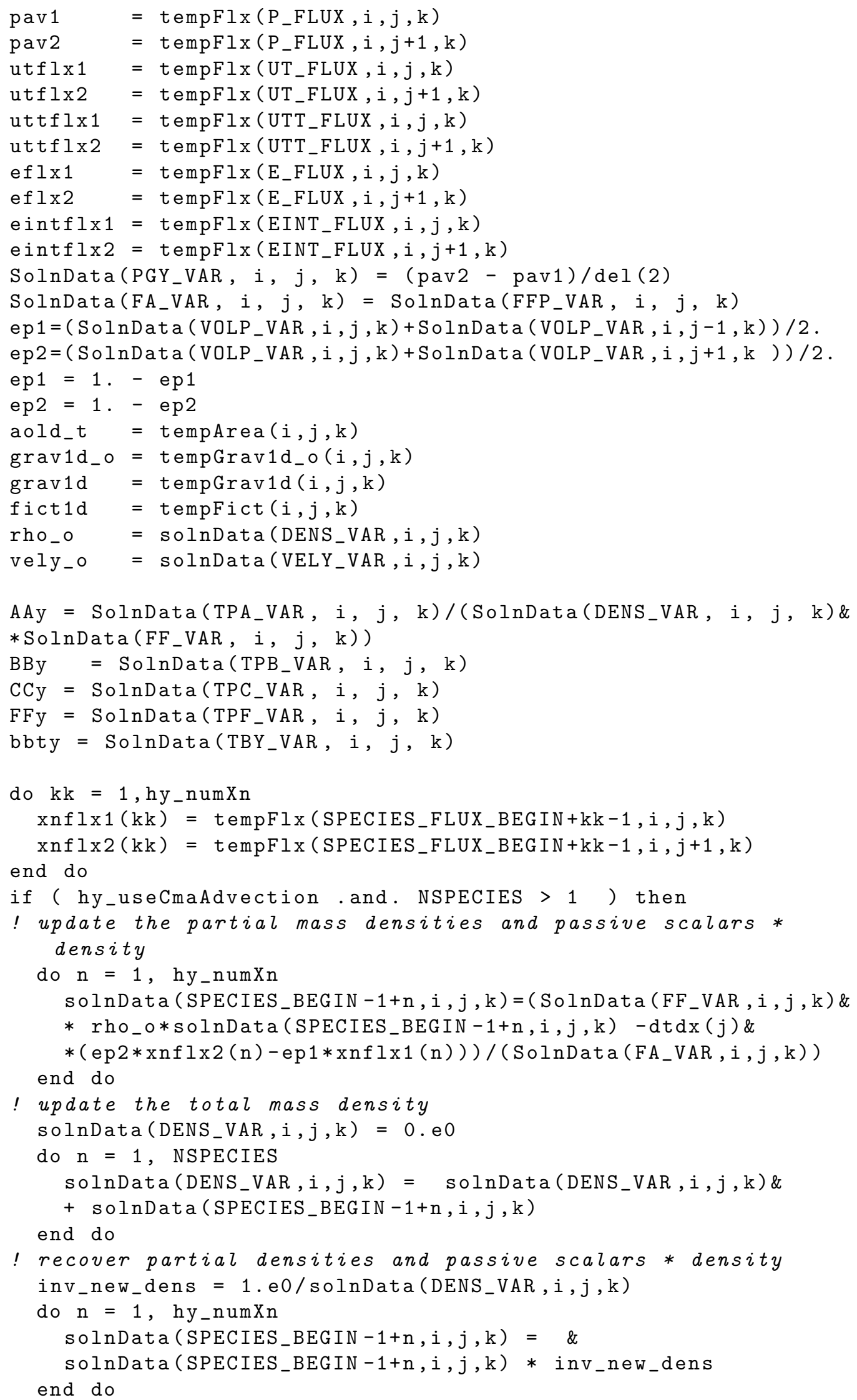




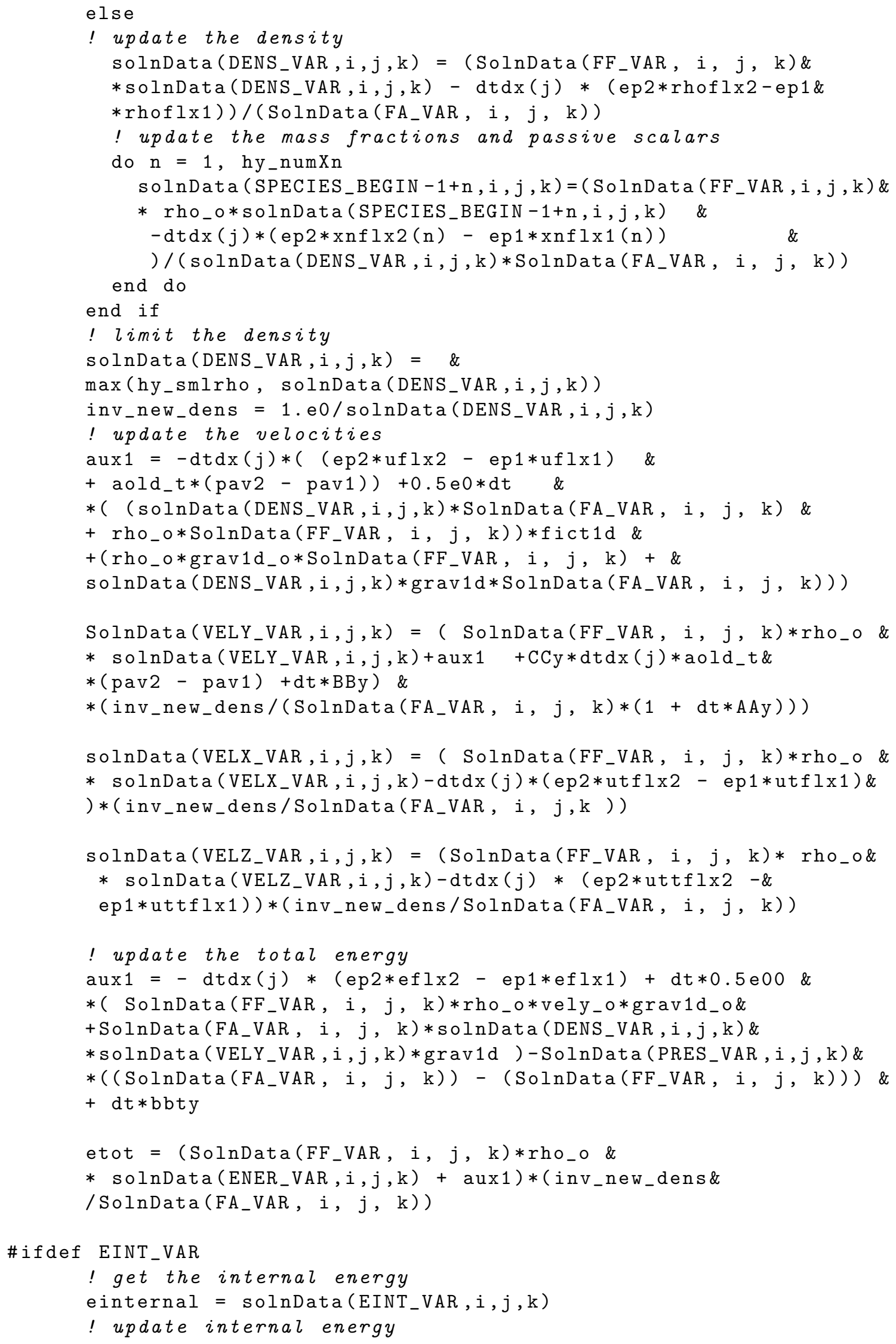




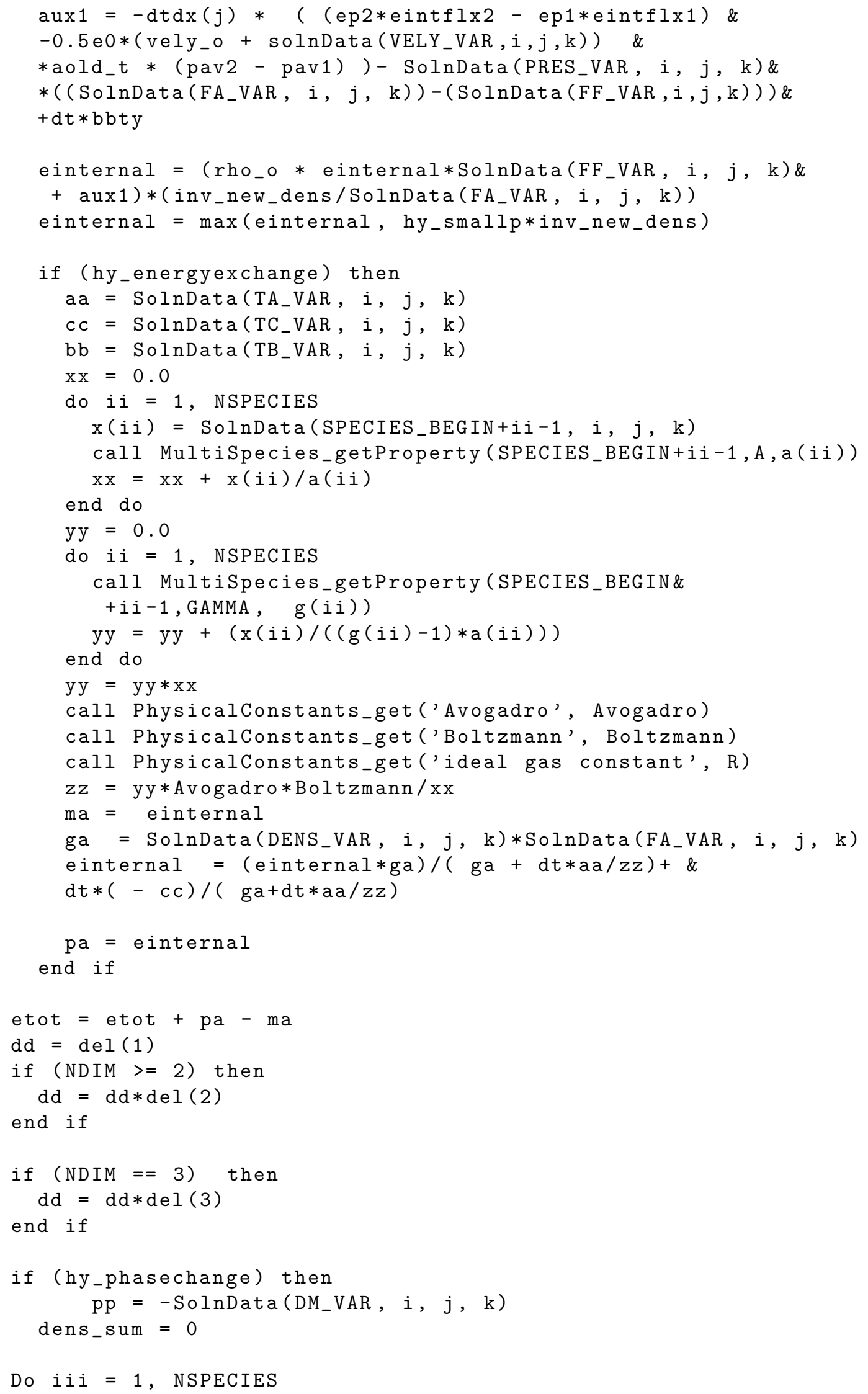




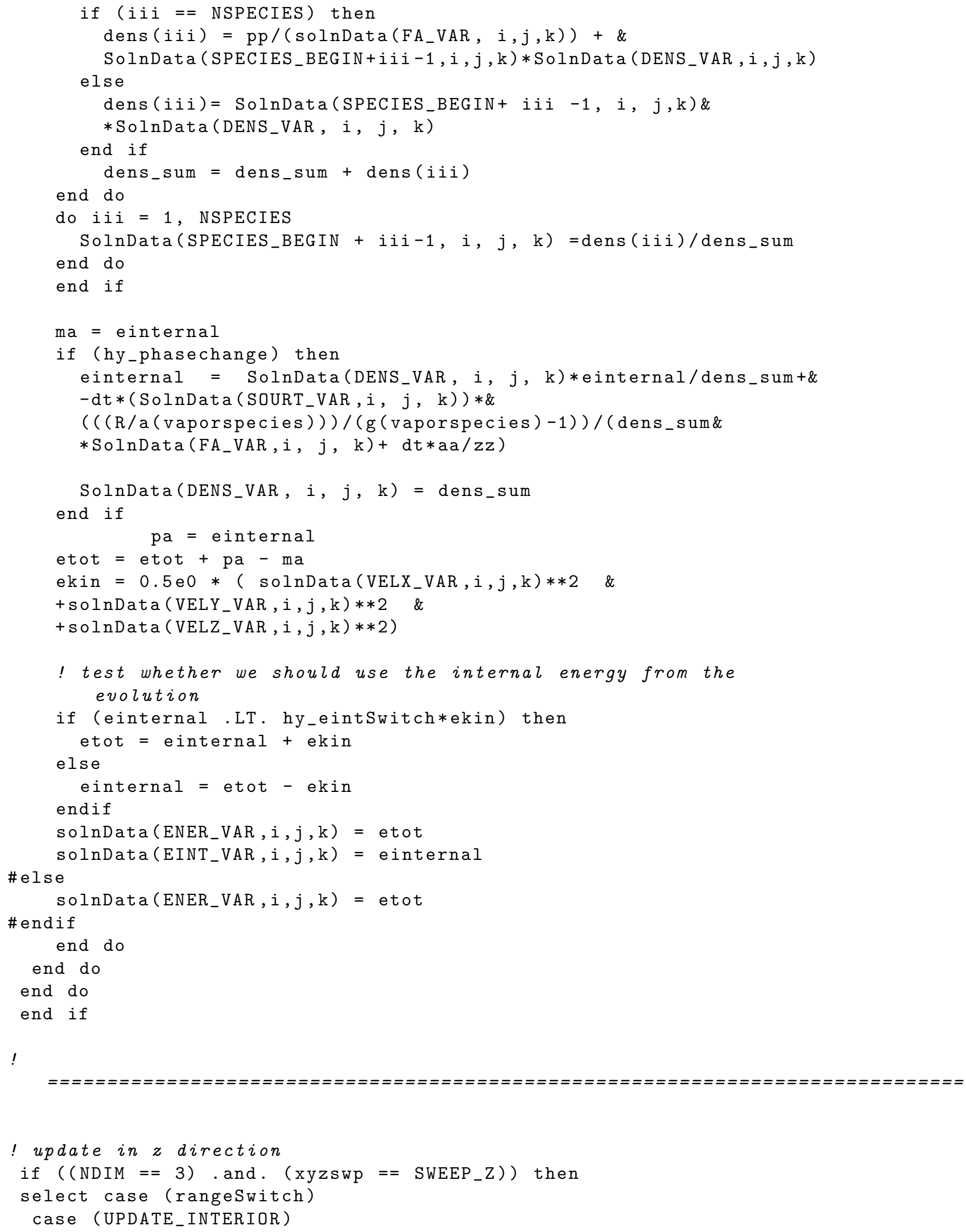




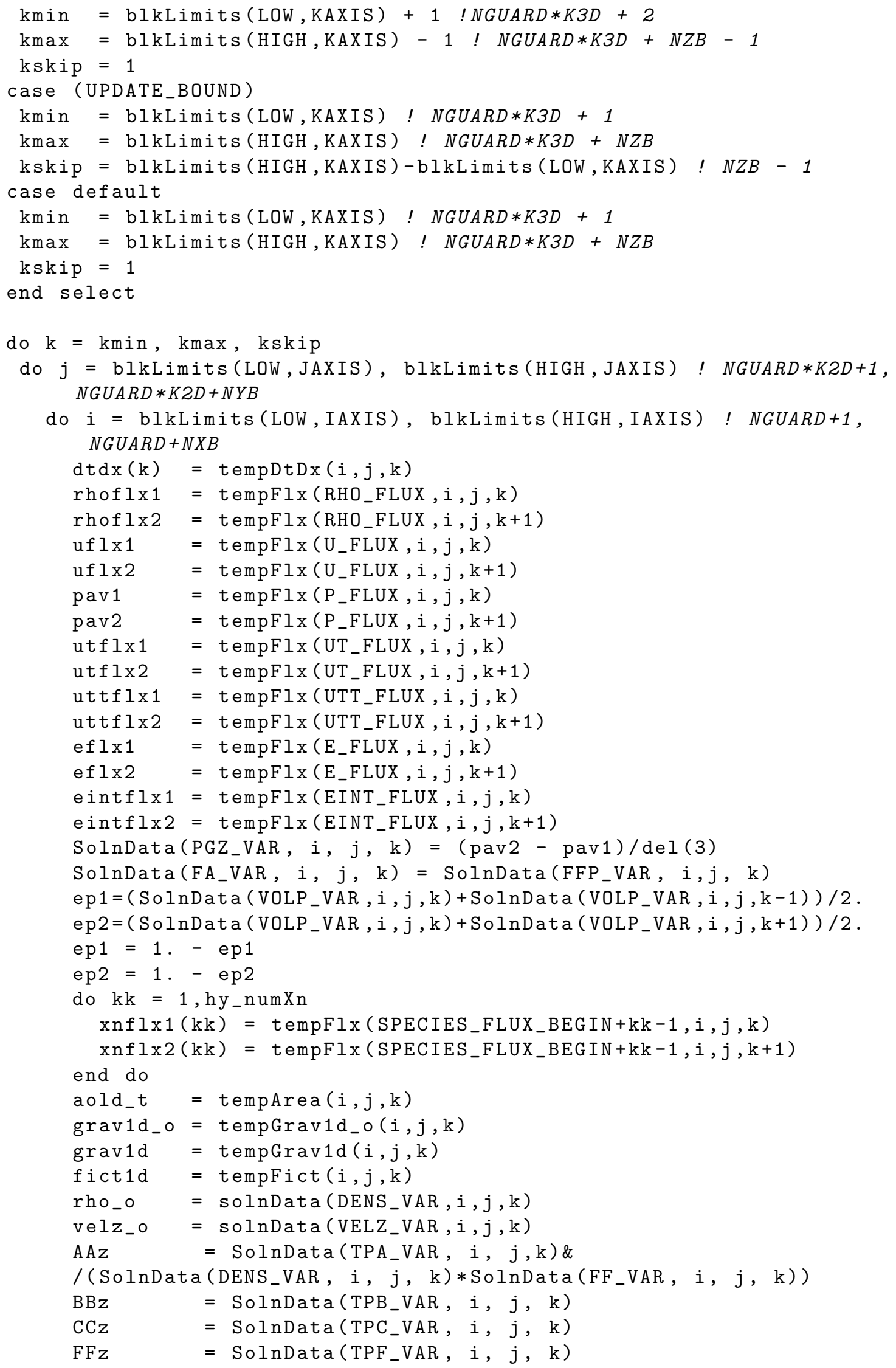




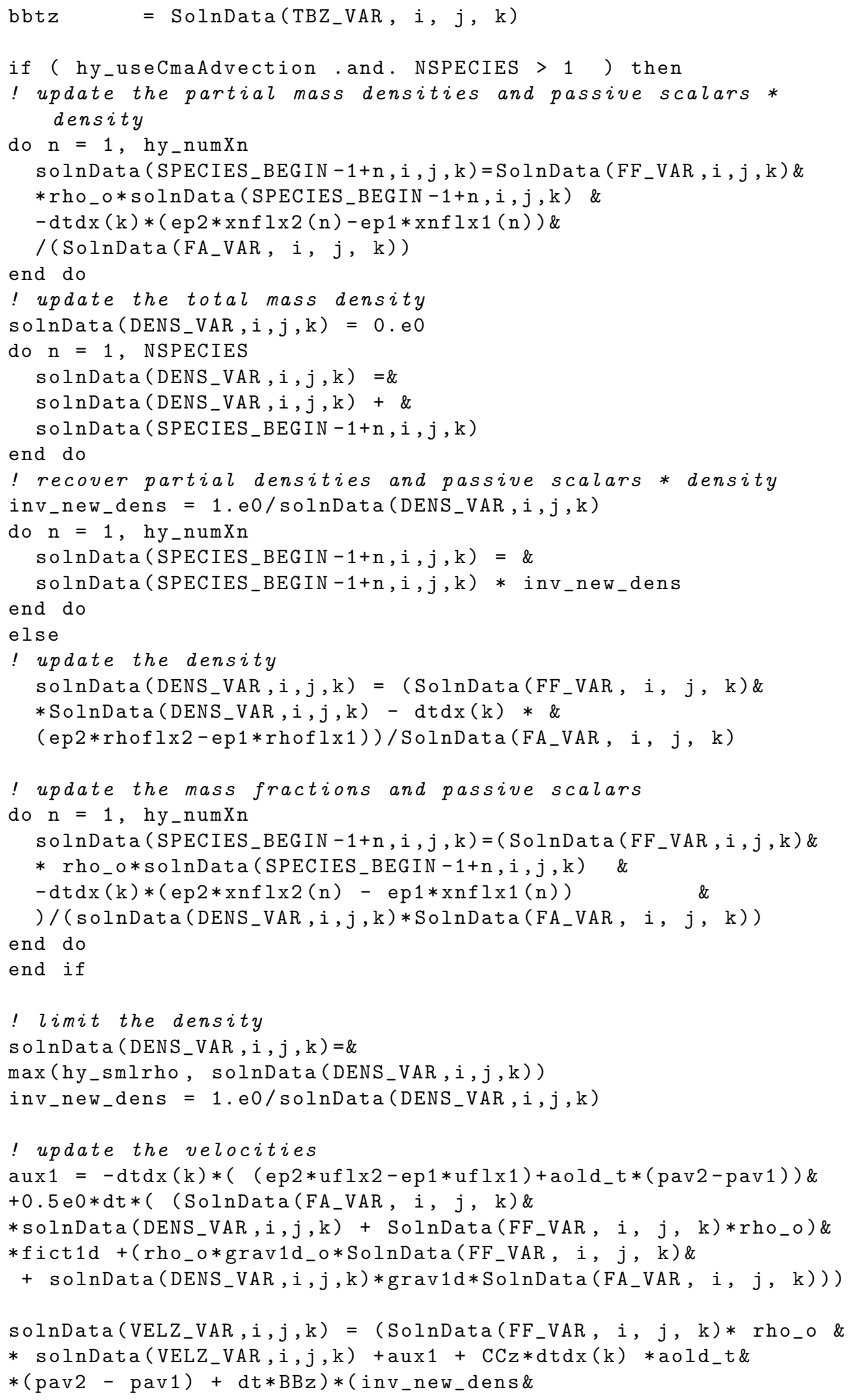




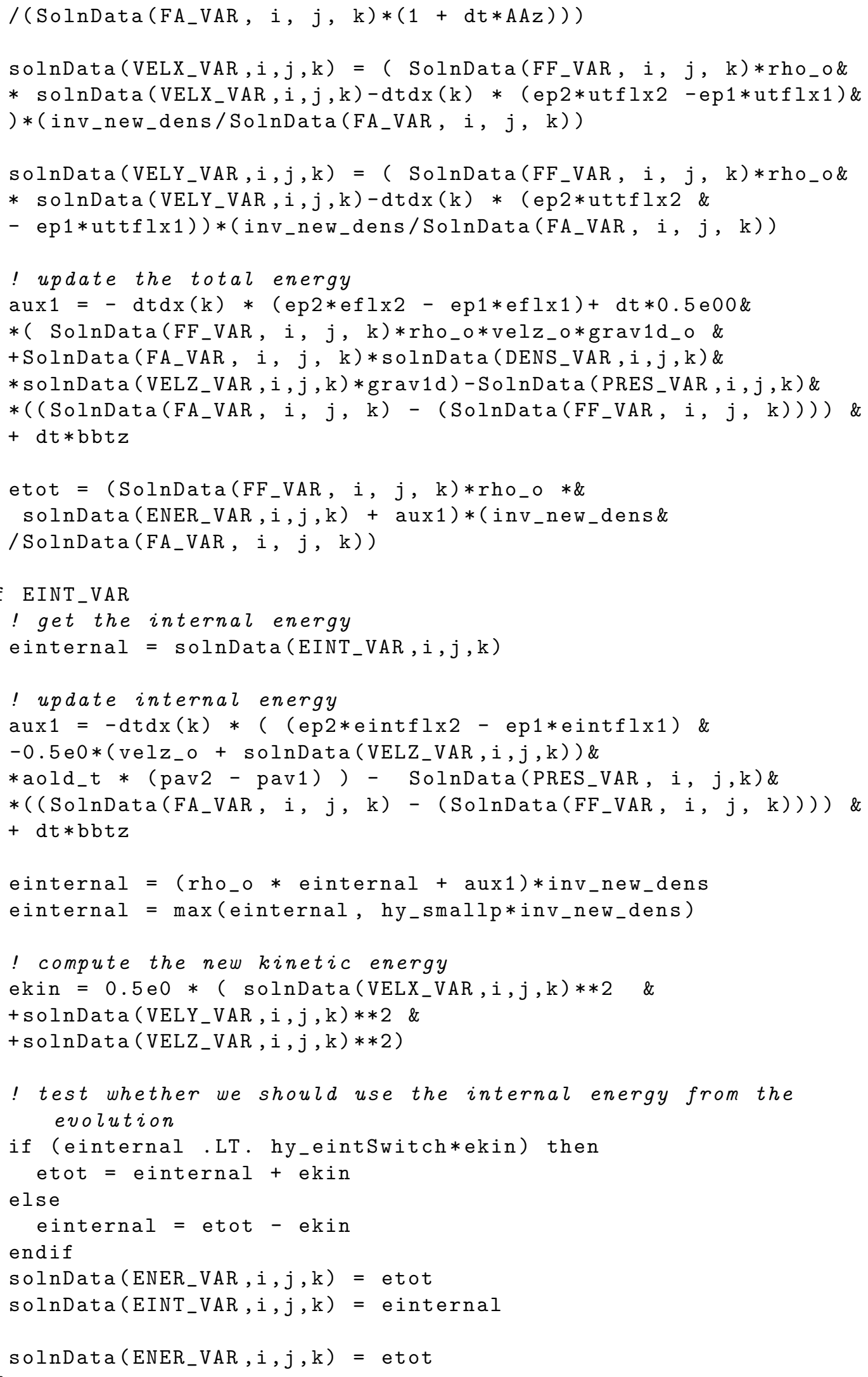




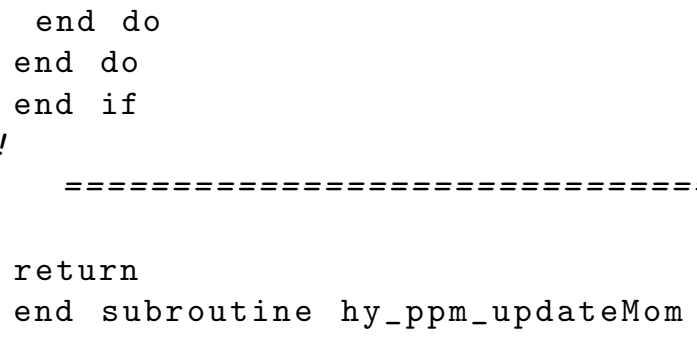

\section{A.4.3 hy_ppm_sweep.F90}

In this subroutine, only a call statement to the hydro solution file is changed. The places where originally the routine calls hy_ppm_updateSoln, our modified subroutine calls hy_ppm_update_mom. 